\title{
Novel approaches to measure physiological and behavioral responses to stress
}

\author{
Dissertation \\ for the award of the degree \\ 'Doctor rerum naturalium' (Dr. rer. nat.) \\ of the Georg-August-Universität Göttingen
}

within the Systems Neuroscience doctoral program of the

Göttingen Graduate School for Neurosciences, Biophysics, and Molecular Biosciences (GGNB) and of the Georg-August University School of Science (GAUSS)

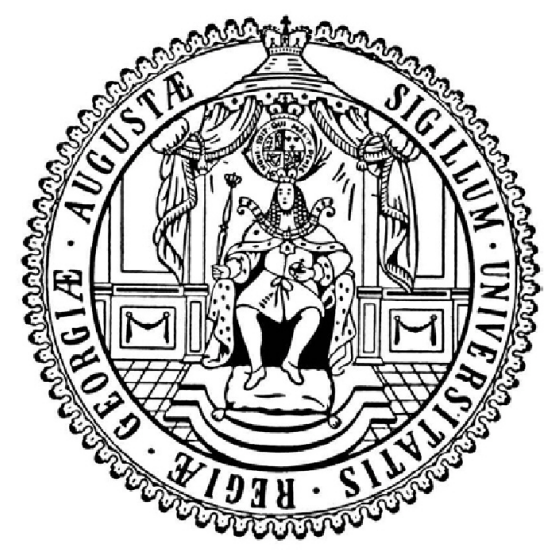

submitted by

Jan Sören Seidel

born in Bad Kreuznach

Göttingen, 2019 



\section{THESIS ADVISORY COMMITTEE}

Prof. Dr. Dr. Hannelore Ehrenreich (Supervisor)

Clinical Neuroscience, Max Planck Institute of Experimental Medicine, Göttingen

Prof. Dr. Lars Penke

Georg Elias Müller Institute of Psychology, Georg-August University, Göttingen

Prof. Klaus-Armin Nave, Ph.D.

Neurogenetics, Max Planck Institute of Experimental Medicine, Göttingen

\section{MEMBERS OF THE EXAMINATION BOARD}

Prof. Dr. Dr. Hannelore Ehrenreich (Supervisor, Referee)

Clinical Neuroscience, Max Planck Institute of Experimental Medicine, Göttingen

Prof. Dr. Lars Penke (Co-referee)

Georg Elias Müller Institute of Psychology, Georg-August University, Göttingen

Prof. Klaus-Armin Nave, Ph.D.

Neurogenetics, Max Planck Institute of Experimental Medicine, Göttingen

Prof. Dr. Annekathrin Schacht

Georg Elias Müller Institute of Psychology, Georg-August University, Göttingen

Prof. Dr. Susann Boretius

Functional Imaging, German Primate Center, Göttingen

Prof. Dr. Walter Paulus

Department of Clinical Neurophysiology, University Medical Center, Göttingen

Date of submission of thesis: October $22^{\text {nd }} 2019$

Date of the oral examination: December $2^{\text {nd }} 2019$ 



\section{DeClaration of Authorship}

I hereby declare that the thesis "Novel approaches to measure physiological and behavioral responses to stress" has been written independently and with no other sources and aids than quoted.

Jan Sören Seidel

Göttingen, October 22 2019 

Dedicated to Ida, Emil, $\mathcal{E}$ Anton

may you always see the world as it should be seen full of wonders 



\section{ACKNOWLEDGEMENTS}

First and foremost I want to thank my supervisor, Prof. Hannelore Ehrenreich, for the opportunity to pursue research in the fascinating field of Clinical Neurosciences. Her supportive attitude, her guidance, and the way she approaches research questions and associated issues have always been a great source of inspiration for me.

Further, I am grateful for all the scientific input given by my Thesis Advisory Committee members, Prof. Lars Penke and Prof. Klaus-Armin Nave, that improved the quality of my work.

I want to thank PD. Dr. Martin Begemann for the amazing time we spent together. So much I have learned during our trips, the examinations of patients, and our numerous discussions. Your kind nature and your respectful interaction with other people, whether they are patients, colleagues, or just strangers in Café Central, will be kept in good memory.

I am very grateful to Dr. Marina Mitjans, her guidance and help, and, of course, for the great and productive cooperations over the years. Besides others, I highly appreciate her contribution to the Violent Aggression project. Likewise, I want to thank Dr. Vikas Bansal and Fabian Bockhop for their help and assistance.

Further, I thank all the students I had the honor to supervise over the course of my PhD. I hope, I have not been the only one who learned a lot working with you.

Moreover, I want to thank every single one in the Clinical Neuroscience group for all the moments, both great and sometimes tough, we experienced together as a team.

Also, I want to acknowledge the help and contribution of the Light Microscopy Facility team, namely Dr. Mišo Mitkovski, Katharina Schneider, and Heiko Röhse, for their participation in our work on infrared thermography.

And, last, but definitely not least, I want to express my deepest gratitude to my family, my partner, and my friends for all the support, encouragement, and heads-ups given over the years. 



\section{TABLE OF CONTENTS}

1 INTRODUCTION TO THE THESIS WORK 1

1.1 The perception of danger affects body, cognition, and behavior in multiple

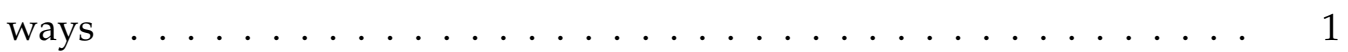

1.2 Stress can have severe implications on medical and psychological wellbeing 2

1.3 Aggression is a natural behavioral response to stressful events . . . . . . . 2

1.4 Aggression is a consequence of the interplay between genetics and the

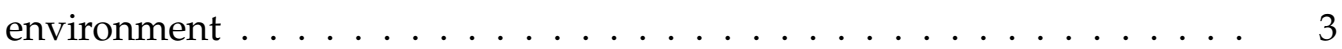

1.5 Schizophrenia is a severe psychiatric condition associated with stress and

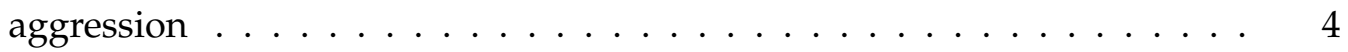

1.6 Stress leads to autonomous responses in animals and humans . . . . . . 5

1.7 Infrared thermography is a non-invasive, contact-free technique to measure body surface temperature $\ldots \ldots \ldots \ldots \ldots$

1.8 IRT has several advantages over 'conventional' physiological measurement techniques ......................... 11

1.9 Limitations of modern IRT in research context $\ldots \ldots \ldots$

2 SCOPE OF THE PRESENT WORK $\quad 15$

3 Project I: Violent AggRession PREdicted by MUltiple PRE-AdUlt ENVi$\begin{array}{ll}\text { RONMENTAL HITS } & 17\end{array}$

3.1 Overview of project I . . . . . . . . . . . . . . . 17

3.2 Original publication $\ldots \ldots \ldots \ldots \ldots$

4 Project II: VAscular Response to social Cognitive Performance MeaSURED BY INFRARED THERMOGRAPHY: A TRANSLATIONAL STUDY FROM MOUSE $\begin{array}{ll}\text { TO MAN } & 37\end{array}$

4.1 Overview of project II $\ldots \ldots \ldots \ldots \ldots \ldots \ldots$

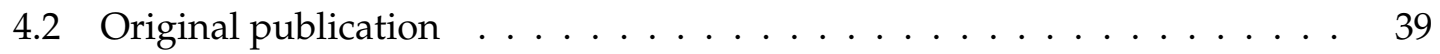

5 SUMMARY AND CONCLUSIONS 
A Appendix

A.1 Accepted co-authorships . . . . . . . . . . . . . . . . . . . . . 69

A.2 List of publications $\ldots \ldots \ldots \ldots \ldots \ldots \ldots$ 


\section{LIST OF FIGURES}

1.1 Example of the process of IRT recording and following data extraction . . 9

1.2 Facial areas exhibit different thermal patterns over the course of IRT _ . . 11 



\title{
List OF ABbREVIATIONS
}

\author{
$\& \quad$ and \\ ${ }^{\circ} \mathbf{C} \quad$ degree Celsius \\ ${ }^{\circ} \mathbf{K} \quad$ degree Kelvin \\ $\mu$ micrometer \\ ANS autonomous nervous system \\ CGAS candidate gene association study/studies \\ DNA deoxyribonucleic acid \\ e.g. for example (abbr. of latin: exempli gratia) \\ et al. and others (abbr. of latin: et alii) \\ FRT Face Recognition Test \\ GRAS Göttingen Research Association for Schizophrenia \\ GWAS genome-wide association study/studies \\ HDAC histone deacetylase \\ HPA axis hypothalamic-pituitary-adrenal axis \\ i.e. that is (abbr. of latin: id est) \\ IRT infrared thermography \\ MAOA monoamine oxidase A \\ mK millikelvin \\ mRNA messenger ribonucleic acid \\ PTSD posttraumatic stress disorder \\ VASS Violent Aggression Severity Score \\ VTNR variant number tandem repeat \\ WCST Wisconsin Card Sorting Test
}





\section{INTRODUCTION TO THE THESIS WORK}

What and who we are is determined by numerous factors. Besides hereditary traits, the experiences we make over the course of our lives affect us in both positive, but sometimes also negative ways. Stress, defined as the actual or anticipated perception of a threat to homeostasis or wellbeing (Ulrich-Lai \& Herman, 2009), plays an important role in an organism's ability to adapt to adverse environmental events. In order to mitigate the impact of hazardous conditions, it is crucial to show an adequate physiological and behavioral stress response (Hollon, Burgeno, \& Phillips, 2015).

\subsection{The perception of danger affects body, cognition, and behav- ior in multiple ways}

Threats to one's physical or psychological integrity come in different flavors (Bhatnagar, Vining, Iyer, \& Kinni, 2006; Juruena, 2014). Their perception typically leads to certain alterations of endogenous processes in order to initiate adaptive behavioral actions and to restore homeostasis (Smith \& Vale, 2006). In rodents and humans, the hypothalamicpituitary-adrenal (HPA) axis is most relevant in mediating stress responses. As soon as a potential threat is detected, the paraventricular nucleus of the hypothalamus releases both corticotropin-releasing hormone and vasopressin, triggering the production and secretion of adrenocorticotropic hormone by the anterior pituitary gland and, ultimately, of glucocorticoids as well as mineralocorticoids by the adrenal cortex (Aguilera, 2011; Joseph \& Whirledge, 2017; Smith \& Vale, 2006). This endocrinological cascade is associated with many changes in the peripheral and central nervous system in order to prepare for autonomous and behavioral responses (Ulrich-Lai \& Herman, 2009).

During the stress responses, to increase chances of survival, important body functions, such as the cardiovascular activity, respiration, or metabolic rate, are enhanced, while other, less critical functions are inhibited (Joseph \& Whirledge, 2017). Furthermore, to prevent the risk of lethal blood loss, autonomous nervous system (ANS)-regulated vasoresponses change the volume of arterial blood vessels, typically decreasing blood flow to peripheral body parts (Engert et al., 2014). Physiological research takes advantage of such alterations 
in ANS activity to gain insight into the organism's stress response (Cardone \& Merla, 2017; Ermatinger, Brugger, \& Burkart, 2019). Moreover, levels of hormones that are released via the HPA axis can be analyzed to determine the intensity of perceived stress. In particular glucocorticoids, as the downstream product of the HPA axis (i.e., cortisol in humans, corticosterone in mice and rats), are commonly employed as biological stress markers (Boehringer et al., 2015; Hellhammer, Wust, \& Kudielka, 2009).

\subsection{Stress can have severe implications on medical and psycho- logical wellbeing}

While stress is commonly perceived as unpleasant and adverse, it fulfills an evolutionary purpose and can enhance our physical and psychological functionality (Hollon et al., 2015; McEwen, 2007). However, intense or long-lasting periods of stress may have a negative impact on, or may even be the cause of, somatic diseases as well as psychological and cognitive states (Hollon et al., 2015; Yaribeygi, Panahi, Sahraei, Johnston, \& Sahebkar, 2017). Stress is further linked to dysfunctional behavioral patterns and unfavorable coping mechanisms, such as substance abuse (Piazza \& Le Moal, 1998). With regard to psychiatric conditions, stressful experiences have an at least modulating impact on the development and course of many, if not all, psychiatric syndromes, including posttraumatic stress disorder (PTSD), major depression, and schizophrenia (Agid, Kohn, \& Lerer, 2000).

\subsection{Aggression is a natural behavioral response to stressful events}

Stress typically leads to characteristic physiological and behavioral responses. One important possible reaction to a threat is aggression, exhibited by many different species, with humans being no exception (Veroude et al., 2016; Waltes, Chiocchetti, \& Freitag, 2016). In general, aggression can be defined as an overt behavior that has the intention of inflicting physical damage or harm to other living beings (Miczek, Fish, De Bold, \& De Almeida, 2002; Nelson \& Trainor, 2007). However, former work suggests distinct subgroups of aggression (e.g., reactive versus proactive; overt versus covert; physical versus relational), with variations in the incidence of these subtypes in relation to context, age, and gender (Björkqvist, 2018; Österman et al., 1998; Veroude et al., 2016). While adaptive in certain situations, when exhibited repeatedly, over longer phases, or out of context aggression 
constitutes a serious problem for society as a whole, both due to individual consequences and economical costs (Blair, 2016). Thus, a closer look at factors modulating the expression of aggressive responses to stress is desirable.

\subsection{Aggression is a consequence of the interplay between genet- ics and the environment}

Over the last decades, genetic and environmental factors that may cause or modulate aggression have extensively been investigated. For instance, twin and adoption studies emphasize that, besides a strong hereditary impact, the relevance of environmental factors should not be underestimated. In particular, the non-shared environment plays an at least moderate role in aggressive behavior. However, due to differences in age and gender of the investigated individuals as well as operationalization of aggression phenotypes over studies, the amount of estimated genetic and environmental influence varies (Veroude et al., 2016). Accordingly, genome-wide association studies (GWAS), which aimed to identify gene variants relevant for aggressive phenotypes, could not achieve genome-wide significances (Mick et al., 2014; Tiihonen et al., 2015; Waltes et al., 2016). Approaches of candidate gene association studies (CGAS), on the other hand, targeted and identified several genes possibly connected to aggression. In particular, genes that influence monoaminergic neurotransmission (Veroude et al., 2016; Waltes et al., 2016) seem to be of importance.

Research genuinely relying on genetic readouts neglects the interplay between genes and the environment, which seems to serve as an important modulator of the development of aggressive behavior and related disorders. In an influential study, Caspi and colleagues (2002) prospectively examined the impact of a variant number tandem repeat (VNTR) polymorphism in the promoter region of the monoamine oxidase A (MAOA) gene on the occurrence of antisocial problems in men. The $M A O A$ gene is located on the $\mathrm{X}$ chromosome and expresses an enzyme that metabolizes monoamines, such as serotonin, dopamine, or noradrenaline. These neurotransmitters have been linked to aggression (Brunner, Nelen, Breakefield, Ropers, \& van Oost, 1993). In 2002, Caspi et al. reported that low MAOA activity, due to the VNTR polymorphism, is linked to a higher risk of antisocial behavior. However, this higher risk could only be observed in individuals who additionally suffered 
from maltreatment during childhood, highlighting the importance of both genes and adverse environmental factors in the explanation of problematic behavior patterns (Caspi et al., 2002). Their findings have been replicated multiple times (Byrd \& Manuck, 2014; Kim-Cohen et al., 2006), suggesting a robust gene $\times$ environment interaction effect on aggression. Epigenetic analyses try to combine both genetic and environmental research by investigating the effects of environmental factors on the expression of genes via, besides others, DNA methylation, chromatin modification, or histone acetylation and deacetylation (Palumbo, Mariotti, Iofrida, \& Pellegrini, 2018).

Studies examined a huge number of potentially relevant environmental risk factors with respect to aggressive behavior. However, such work often focuses on not clearly defined environmental experiences (e.g., childhood maltreatment). More importantly, risk factors are often assessed in an isolated manner, ignoring possible additive effects (Raine, 2002). Furthermore, several other parameters moderate aggressive behavior, such as age and gender (Björkqvist, 2018; Österman et al., 1998). Moreover, an altered state of mind, typically found in psychiatric conditions, such as schizophrenia, is prominently linked to increased aggressive potential. In fact, aggression does occur more frequently in patients with schizophrenia compared to individuals without. Yet, just a minority of patients actually exhibits aggressive behavior, often moderated by additional factors (Fazel, Gulati, Linsell, Geddes, \& Grann, 2009; Sariaslan, Lichtenstein, Larsson, \& Fazel, 2016).

\subsection{Schizophrenia is a severe psychiatric condition associated with stress and aggression}

Schizophrenia is a chronic mental disease, which usually starts in early adulthood and severely impairs cognitive functions, affect, and behavior of patients. It is characterized by both positive symptoms, like sensory hallucinations, delusions, and disordered thoughts as well as negative symptoms, such as poverty of speech (alogia), reduction of emotions and motivation, and social withdrawal (American Psychological Association, 2013; Howes \& Murray, 2014). With a lifetime prevalence of approximately $1 \%$, schizophrenia is one of the very common psychiatric disorders, with highly heterogeneous interindividual disease patterns (Howes \& Murray, 2014). Schizophrenia negatively affects an individual's 
ability of daily living and quality of life in manifold ways. Moreover, due to high direct and indirect costs, schizophrenia is not only a burden for patients and their personal environment but also for society (Knapp, Mangalore, \& Simon, 2004; van Os \& Kapur, 2009).

Both genetic and environmental risk factors have been associated with schizophrenia (Belbasis et al., 2018; Schizophrenia Working Group of the Psychiatric Genomics Consortium, 2014; Brown, 2011; van Os \& Kapur, 2009), but our knowledge concerning its development is limited. As an approach to overcome weaknesses of many genetic and environmental studies, our group established the Göttingen Research Association for Schizophrenia (GRAS) data collection as a multicenter assessment of patients suffering from schizophrenia or schizoaffective disorder (Begemann et al., 2010; Ribbe et al., 2010). In a recent study, our group used the potential of the GRAS data collection to investigate the impact of well-defined environmental risk factors on the age of disease onset in a sample of 750 male patients with schizophrenia (Stepniak et al., 2014). We reported that the amount of adverse risk factors experienced before adulthood is strongly related to the age of onset of schizophrenia. Moreover, extreme group comparisons revealed a 10 years earlier disease onset in patients with a high, in relation to those with a low, risk load. Contrary to the remarkable effects of the environment on disease onset, polygenic risk scores did not reveal notable results. Having these large additive environmental effects on the development of schizophrenia in mind, we were wondering whether models of accumulated environmental risk factors, experienced in critical phases of development (i.e., before adulthood) could improve our understanding of the relationship between environmental stressors and aggression in both individuals from the general population and patients suffering from schizophrenia.

\subsection{Stress leads to autonomous responses in animals and humans}

As discussed before, autonomic responses to negative environmental experiences are an important readout of stress, not only in animal studies but also in human research (Ulrich-Lai \& Herman, 2009). Stress is not only relevant when it occurs in an intense way or in critical stages of development. Chronic mild stress can influence our somatic and 
psychological wellbeing and daily life functioning (Won \& Kim, 2016; Yaribeygi et al., 2017). Therefore, it is worthwhile to take a closer look at the physiological and behavioral responses to more subtle forms of stress.

Animal models of stress commonly rely on the assessment of behavioral reactions within stress-inducing settings. Depending on the experimental scenario, different behavior can be observed, including fight-or-flight reactions, freezing, or situational avoidance (Benus, Koolhaas, \& van Oortmerssen, 1992; de Boer, 2018; Harro, 2018; Toth \& Neumann, 2013). In addition, to get deeper insights into endogenous processes, techniques to investigate physiological reactions to stress are available. The latter range from blood corticosterone levels and core body temperature, often measured via a surgically introduced probe, to posthumous analyses of hypothalamic Fos expression (Bhatnagar et al., 2006; Keeney, Hogg, \& Marsden, 2001; Martinez, Carvalho-Netto, Amaral, Nunes-de Souza, \& Canteras, 2008). Such measures, however, are very invasive or distress animals even further, leading to unnatural testing conditions and thus likely biasing the outcome of experiments (Cardone \& Merla, 2017). Moreover, invasive assessment techniques raise ethical concerns regarding animal welfare (Gjendal, Franco, Ottesen, Sorensen, \& Olsson, 2018; Meyer, Ootsuka, \& Romanovsky, 2017).

Compared to animals, human research provides the great advantage to get insight into psychological states by simply asking. Assuming proper usage, psychometric tools, such as psychological questionnaires or interviews, are known to be capable of collecting valid information on an individual's internal processes. However, subjective self-descriptions may not always be the best choice to investigate stress. For instance, the administration of psychometric instruments is not always feasible over the course of an experiment. Furthermore, given answers are commonly biased and are not necessarily in line with data derived from physiological instruments. Therefore, an accompanying implementation of more objective approaches is indicated.

Human body responses to ANS activity are typically measured via a variety of instruments. Modern techniques are capable of accurately capturing a wide spectrum of basal 
physiological functions, such as the heart rate, arterial blood pressure, respiration rate, muscle tone, or electrodermal activity (Cardone \& Merla, 2017; Engert et al., 2014). Although these valuable tools are important in both neuroscientific research and clinical practice, they all have certain limitations (Ermatinger et al., 2019). Many of these techniques require participants to remain as motionless as possible to prevent measurement artifacts and errors, or they need to apply sensors on the test subjects' bodies (Cardone \& Merla, 2017). Such intrusive conditions typically result in an uncomfortable and artificial testing environment. Moreover, certain investigations, such as an exploration of social interactions, are often considerably limited (Cardone \& Merla, 2017; Ioannou, Morris, Baker, Reddy, \& Gallese, 2017). Furthermore, the application of those techniques is highly challenging in individuals who are not able or willing to follow standard measurement protocols, such as patients suffering from psychiatric conditions (Engert et al., 2014).

In summary, methods commonly used in both animal and human stress studies suffer from limitations that weaken and question the validity of reported outcomes. Therefore, besides others, this field of research could substantially benefit from non-invasive, contact-free techniques to reliably measure ANS activity. Modern infrared thermography recording has the potential to fill this gap.

\subsection{Infrared thermography is a non-invasive, contact-free tech- nique to measure body surface temperature}

Infrared thermography (IRT) was first discovered in 1800 by astronomer William Herschel under the term 'Calorific Rays' while investigating specific radiation emitted by the sun (Herschel, 1800; Rogalski, 2012). In order to quantify the physical property commonly perceived as 'heat', IRT utilizes electromagnetic radiation of a characteristic wavelength outside our visible spectrum of light, namely within a bandwidth of 0.75 to $1000 \mu \mathrm{m}$ (Lahiri, Bagavathiappan, Jayakumar, \& Philip, 2012). Every living being, as well as inanimate objects with a temperature higher than $0^{\circ} \mathrm{K}$, emit radiation within this spectrum, where higher wavelengths are linked to lower temperatures (Tattersall, 2016). As an example, mammals', including humans, body surface constantly emits heat, peaking between 8 and $12 \mu \mathrm{m}$. Thus, IRT specialized in recording living beings mostly relies on sensors made of materials sensitive to long-wavelength infrared (Lahiri et al., 2012; Tattersall, 2016). 
Today, infrared radiation technology commonly used to detect thermal alterations relies on either cooled (quantum) or uncooled (thermal) detectors (Usamentiaga et al., 2014). Quantum IRT systems utilize the interaction of semiconductors with changes in the energy distribution of electrons due to the stimulation of thermal photons (Cardone \& Merla, 2017; Rogalski, 2012). They depend on selective wavelengths and achieve very high measurement precision. Given the high demand for constant cooling, this type of detectors are characterized by high costs, a bulky and heavy design, and a complicated way of usage (Rogalski, 2002). Conversely, cameras with uncooled thermal detectors are calibrated to a certain temperature (e.g., ambient temperature) and are typically independent of photonic wavelength (Rogalski, 2012). In order to translate electromagnetic radiation to electricity and, subsequently, to thermal images, special materials that do not block infrared radiation are employed (Cardone \& Merla, 2017; Rogalski, 2002, 2012). When detector parts are hit by thermal radiation, they heat up and alter their electrical properties. In order to create a visible representation of this process, changes in electrical properties can be translated to corresponding pixels. This results in the creation of a monochromatic image with pixel intensities correlating with the respectively detected thermal energy. For a better visual interpretation, monochromatic images can then be converted into false-color images using different color palettes (Alpar \& Krejcar, 2017; Ring \& Ammer, 2012; Tattersall, 2016). An example of the recording and data quantification process can be found in Figure 1.

In relation to cooled systems, uncooled IRT cameras are characterized by lower thermal sensitivity. Nevertheless, modern cameras still achieve a relatively high spatial (up to 1280 $\times 1024$ pixels) and temporal resolution ( $\geq 200$ frames per second) and combine a satisfying thermal sensitivity (Noise-Equivalent Temperature Difference of not more than $30 \mathrm{mK}$ at 30 ${ }^{\circ} \mathrm{C}$ ambient temperature) with a sufficiently high testing accuracy (Cardone \& Merla, 2017).

At present, IRT plays an important role in different fields, such as the military, law enforcement, search and rescue missions, material sciences, and many more (Jarlier et al., 2011; Rogalski, 2012; Tattersall, 2016; Usamentiaga et al., 2014). In medicine, IRT had its first appearance already in the 1960s. However, technical limitations led scientists and physicians to favor more applicable tools (Lahiri et al., 2012; Ring, 2010). In recent years, technological and manufactural advancements resulted in a comeback of IRT in both medical research and diagnostic applications. Nowadays, modern IRT presents 
itself as a valid, relatively inexpensive and easy-to-use measuring method that is already implemented in diverse areas of medicine, including oncology, dentistry, dermatology, endocrinology, epidemiology, or infectiology (Lahiri et al., 2012; Ring \& Ammer, 2012; Usamentiaga et al., 2014).
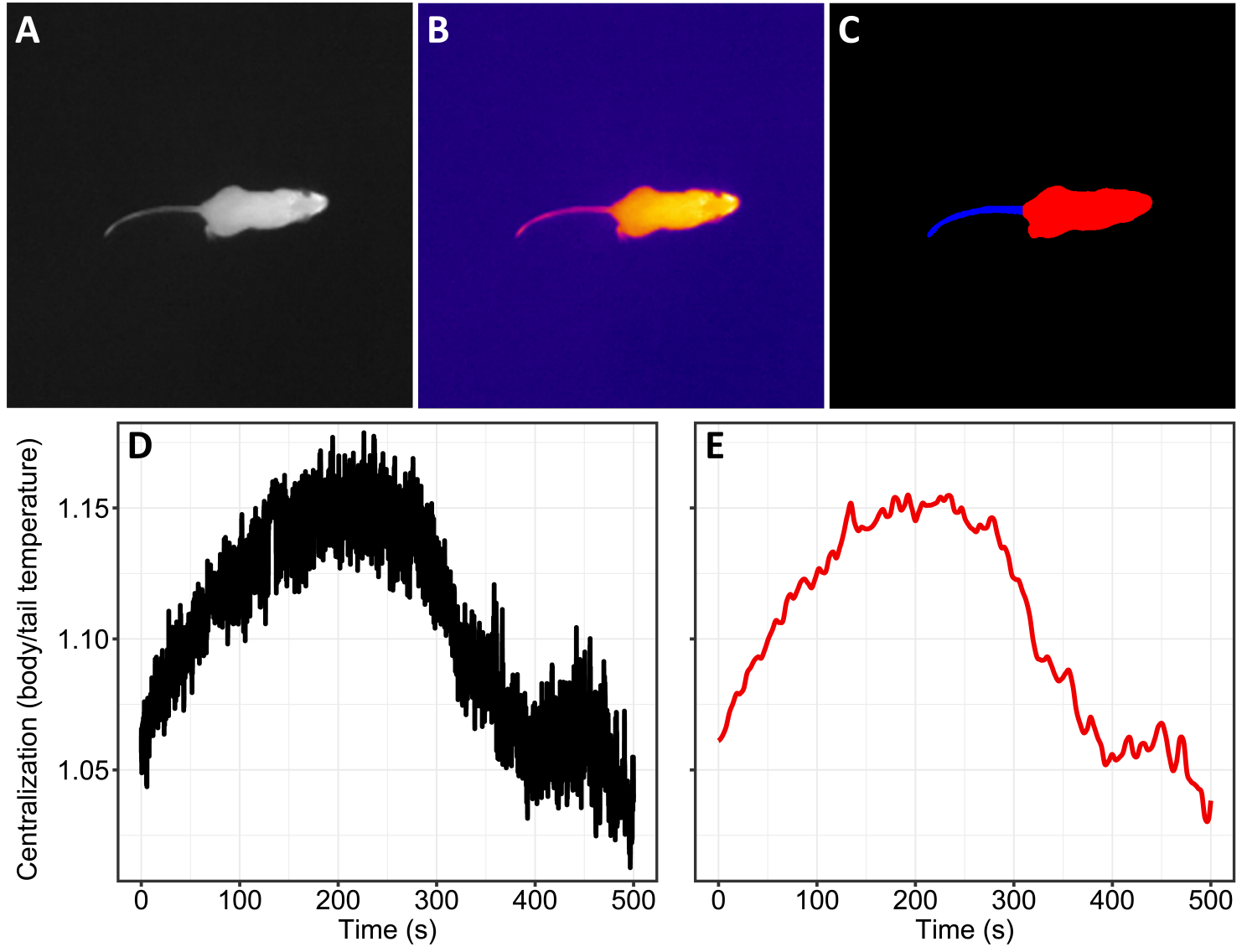

FIGURE 1.1: Example of the process of IRT recording and following data extraction.

A: Grayscale image derived from a mouse performing an Open Field paradigm. B: Pseudo-colors application makes differences in surface temperature easily visible. C: Peripheral (blue) and core (red) body regions are defined for the following data extraction. D: Thermal curve of the index of core body divided by periphery (centralization) over the course of 500 seconds. E: To reduce noise thermal curve can then be smoothed.

Recently, IRT has found its way into neuroscientific research, including both animal and human subjects (Cardone \& Merla, 2017; Ermatinger et al., 2019; Gjendal et al., 2018; Herborn, Jerem, Nager, McKeegan, \& McCafferty, 2018; Ioannou, Gallese, \& Merla, 2014; Moline et al., 2017; Mufford et al., 2016; Tattersall, 2016). Mammals are endothermic organisms and therefore in need of maintaining stable body temperature by internal regulation processes, including muscular activity (e.g., shivering) to increase body heat and perspiration (i.e., activation of sweat glands) in order to cool down (Mufford et al., 2016; Ring, 2010). But body temperature does not only alter in response to physical parameters like ambient temperature changes. Autonomous activity, for instance, as a response to 
stressful experiences, leads to alterations in volumes of blood vessels (i.e., vasoconstriction and vasodilation) in both peripheral and core body regions, culminating in changes in blood flow (flushing; Gjendal et al., 2018; Meyer et al., 2017; Mufford et al., 2016; Yoshihara et al., 2016). Autonomous responses prepare animals for defensive actions in the eye of a physical or predatory threat (e.g., fight-or-flight). Moreover, they provide protection from blood loss due to injury and hence increase the chances of survival (Herborn et al., 2018; Ioannou et al., 2014; Vianna \& Carrive, 2005). As a side effect, such highly adaptive physiological alterations concur with changes in temperature of core and peripheral body parts. Remarkably, IRT is capable of quantifying these changes by recording heat emissions from the body surface.

Accordingly, in humans, associations between physiological processes, detectable via measurements of thermal alterations, and psychological or behavioral reactions have long been reported. For instance, already Darwin (1872) described the relationship between facial flushing and emotions, such as anger. Subsequently, other authors reported vasoconstriction or vasodilation as a result of autonomous activity that is strongly linked to thermal changes in both acral and central body parts as well as facial regions (Ioannou et al., 2014, 2017). Notably, in a series of studies Kistler and colleagues (Kistler et al., 1996; Kistler, Mariauzouls, \& von Berlepsch, 1998a, 1998b) used Laser Doppler Flowmetry and photoplethysmography techniques to associate sympathetic vasoconstriction and temperature alterations in the fingertips during sympathetically activating procedures, such as watching horror movies, chewing cotton swaps, or receiving an acupuncture treatment. The authors showed that with a delay of approximately 15 seconds, temperature changes reliably occurred in more than $90 \%$ of the time. Similarly, Drummond and colleagues used Laser Doppler Flowmetry in order to explore the relationship between blood vessel volume and anger induction in female (Drummond, 1999), and during an embarrassment task in male and female, participants (Drummond \& Su, 2012).

The human face is of particular interest for thermal studies. Due to a complex network of blood vessels under its surface, alterations in ANS activity have strong effects on subcutaneous blood flow and, consequently, temperature in different facial regions (Figure 2; Ioannou et al., 2017). Engert et al. (2014) investigated thermal alterations of various 
areas of the face in response to physical and social stress, revealing large differences in thermal patterns in relation to the type of stressor experienced. Changes in flushing, due to negative or positive social stimuli, combined with different intensity levels also lead to alteration in surface temperature, with, in particular, the nose consistently reported as highly reactive to affective and social cues (Cardone \& Merla, 2017; Engert et al., 2014; Ioannou et al., 2017; Kosonogov et al., 2017).
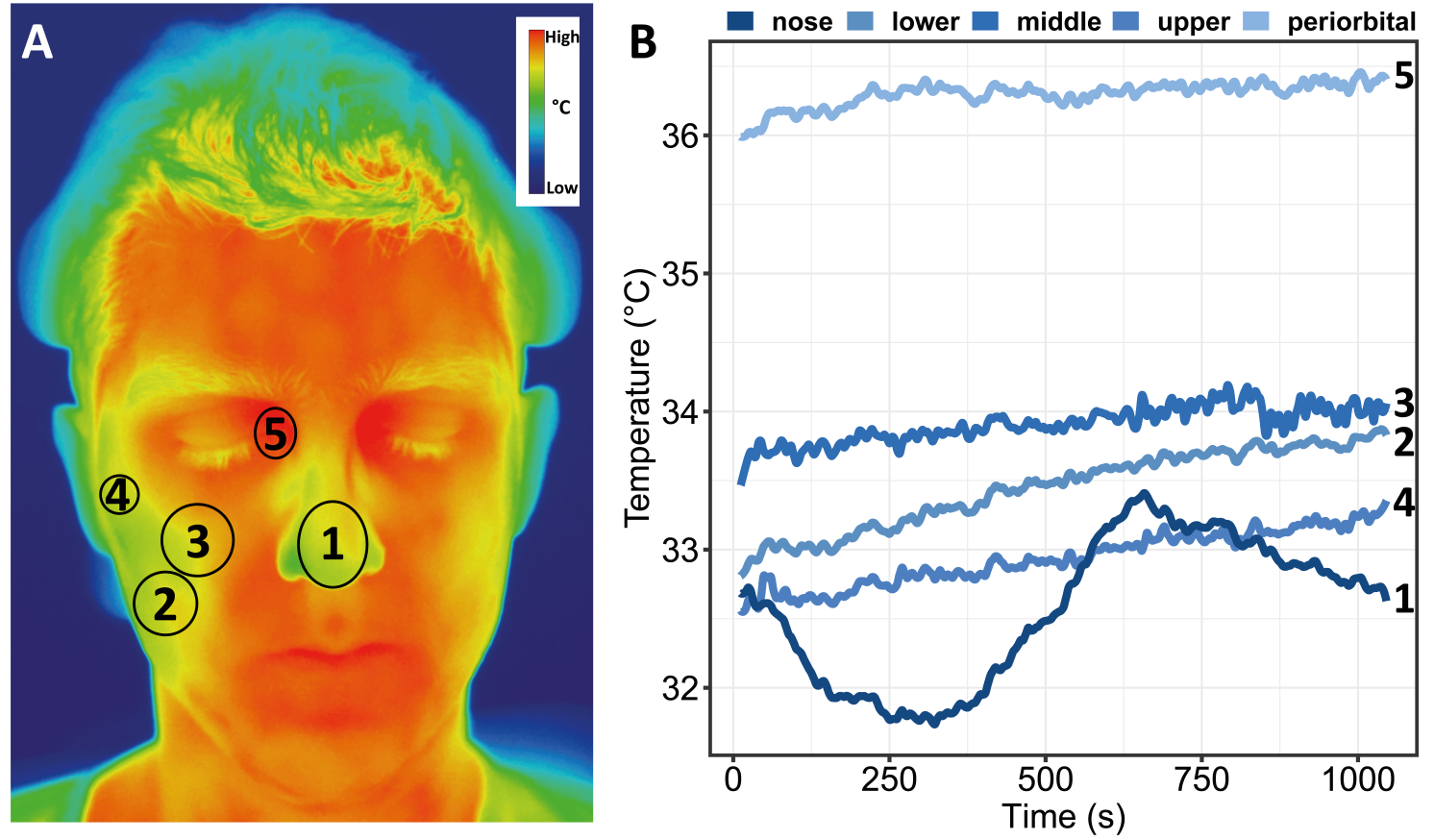

FIGURE 1.2: Facial areas exhibit different thermal patterns over the course of IRT.

A: False-color image of a participant reveals area-specific face surface temperatures. Ellipses denote defined regions of interest for subsequent data extraction, namely nose (1), lower (2), middle (3), and upper (4) cheek, and periorbital area (5). B: Quantification of these regions shows different temperatures and also thermal curves during a cognition task with social components, with the nose exhibiting most reactivity.

\subsection{IRT has several advantages over 'conventional' physiologi- cal measurement techniques}

IRT has been proven to have a satisfactorily high accuracy in measuring physiological parameters, such as cardiac pulse, respiratory rate, and cutaneous blood perfusion rate, as compared to state-of-the-art techniques in humans (Cardone \& Merla, 2017; Cardone, Pinti, \& Merla, 2015; Ermatinger et al., 2019; Ioannou et al., 2014). Additionally, former work showed that IRT is a valid readout of ANS activity in mice (Gjendal et al., 2018). Further, it displays several advantages over other established physiological measuring techniques. 
Most importantly, IRT offers an easy to use, non-invasive, and contact-free application mode. This renders it feasible to conduct experiments with almost no restriction in the movements of test subjects, leading to a more naturalistic and ecological testing environment (Ioannou et al., 2014, 2017). Moreover, IRT can record and analyze multiple test subjects simultaneously, remarkably facilitating research of social contexts, deeming IRT as the most suitable method in this research field (Cardone \& Merla, 2017). IRT is fully functional even in complete darkness (i.e., in absence of visible light). Accordingly, enhanced measurements under conditions with light as a limiting factor, for instance in testings of nocturnal animals, is possible. Recent work successfully introduced IRT into the assessment of psychiatric patients, making it possible to investigate individuals who have difficulties with the restrictive standard measuring procedures of other methods (Di Giacinto, Brunetti, Sepede, Ferretti, \& Merla, 2014; Jian, Chen, Chu, \& Huang, 2017; Perpetuini et al., 2019). Taken together, IRT can be seen as a valuable, non-invasive, economical tool to conduct psychophysiological research in more naturalistic contexts.

\subsection{Limitations of modern IRT in research context}

Even though IRT's validity in displaying surface temperature is generally accepted, its testing reliability depends on controlling of numerous environmental, personal, and technological factors (Fernández-Cuevas et al., 2015). Physical parameters, including ambient temperature and humidity, direct sunlight, or emission from other sources of heat, interfere with IRT. To obtain proper recordings, IRT cameras also need a clear and direct focus on the relevant body surfaces. Moreover, multiple personal factors, comprising gender, age, amount of body fat, diurnal or menstrual cycle, food intake or consumption of psychoactive substances, besides others, display potentially confounding effects and, therefore, have to be taken into account.

Unfortunately, many former studies did not sufficiently control for these issues. Moreover, no overall accepted gold standard in IRT testing has been introduced yet. Hence, numerous different experimental designs, test stimuli, and examined facial regions as well as ways of data extraction and analysis have been utilized (Ermatinger et al., 2019; Ioannou et al., 2014; Ring \& Ammer, 2012). Rather small and heterogeneous sample groups further limit 
explanatory power. The majority of previous work relies on the analysis of single or short series of IRT images (e.g., before versus after experimental manipulation), examinations of thermal dynamics over the course of longer-lasting time intervals are scarce. Thus, the interpretation of IRT alterations is often limited to unidirectional statements. 


\section{SCOPE OF THE PRESENT WORK}

As discussed in the Introduction, stress is long known to be an internal reaction in the face of danger or threat to physiological or psychological integrity. Typically, stress results in adaptive physiological and behavioral responses, aiming at reducing the impact of perceived threats and, ultimately, to re-establish and maintain homeostasis. Apart from that, chronic or intensive stress experiences, particularly in critical periods of development, can have a negative impact on an individual's physical and psychological health. Furthermore, stress commonly accounts for a broad spectrum of dysfunctional behaviors. Aggression, due to its impact on individuals and society, is a very prominent target of respective investigations. Different models have been postulated to explain the association between stressors and aggression, still, most work focuses on individual risk factors. This weakens power in explaining or predicting aggressive behavior. Likewise, a range of measurement techniques can utilize autonomous responses to stressful experiences. These techniques are invasive, demand applications of body sensors, or require test subjects to remain unnaturally motionless. Such limitations, besides others, may greatly bias the context of measurements. Infrared thermography (IRT) can overcome these issues. However, due to differing quality in controlling for external and personal confounding factors as well as study designs, findings reported from former IRT studies have to be interpreted with caution.

This work seeks to address the discussed issues of former research, using two innovative methodological approaches. Within the first research project, we create a robust model of accumulated environmental risk to predict violent aggression in large groups of both schizophrenia patients and individuals from the general population in several independent samples. The second project aims to enhance the methodological quality of IRT as a reliable tool in both mice and humans to measure autonomic responses to stress and arousal by utilizing novel approaches in IRT data acquisition, preprocessing, and analyses. 


\section{Project I: Violent AgGRESSiOn PREDicted BY}

\section{MULTIPLE PRE-ADULT ENVIRONMENTAL HITS}

\subsection{Overview of project I}

Aggression is of great concern for individuals and society as a whole. The identification of factors playing a vital role in its manifestation is useful with respect to the prevention of aggression. Unfortunately, previous research lacks empirically confirmed models capable of predicting aggressive behavior. Notable work highlights the substantial impact of the environment in the development of aggression and antisocial behavior (Caspi et al., 2002; Nelson \& Trainor, 2007; Palumbo et al., 2018; Veroude et al., 2016; Waltes et al., 2016). However, studies focusing solely on genetic data (e.g., classical GWAS) neglect the important contribution of the environment to the occurrence of aggressive behavior. Conversely, phenotypical studies attempt to elucidate the role of the environment in aggression. However, such research commonly investigates specific, individual factors instead of assessing a possible additive effect (Raine, 2002).

Aggressive behavior is commonly attributed to patients suffering from mental disorders, such as schizophrenia (Fazel et al., 2009; Sariaslan et al., 2016). Albeit the fact that aggression occurs more often in patients with this disorder, compared to individuals without (Fazel et al., 2009; Sariaslan et al., 2016), the relevance of moderating factors remains rather obscure. Our study was conducted to improve our knowledge about negative environmental influences on violent aggression.

In a follow-up to the study by Stepniak et al. (2014), we planned to compare male schizophrenia patients with low and high amounts of adverse environmental experiences before adulthood in a highly matched discovery sample. In doing so, we realized that high-risk patients had a great chance of a record of past or current aggressive behaviors. Thus, we were wondering about a possible connection between adverse environmental risks and the likelihood of displaying aggressive behavior. 
As a first approach, we carefully screened all information on the discovery sample patients, collected during GRAS examinations (Begemann et al., 2010; Ribbe et al., 2010), with respect to any aggressive behavior. Based on these data, we created the Violent Aggression Severity Score (VASS) as a highly sensitive readout of any kind of aggressive behavior over the course of the patients' lifetime. Next, we defined environmental risk factors and assessed their relationship with the VASS. In particular, six factors, namely urbanicity (being raised in a big city), migration, physical abuse, sexual abuse, problematic alcohol use, and lifetime consumption of cannabis, revealed effects on aggressive behavior. This observation was consistently reproduced in three independent schizophrenia samples, using a proxy of violent behavior as the target readout. Consequently, we created a model of accumulated risk including the above-mentioned six factors.

We found that, although single risk factors already had a moderate impact on violent aggression readouts, our accumulation models revealed strong associations between violent behavior in relation to the number of risk factors. Interestingly, the described risk was only observed in patients who suffered from such negative experiences before adulthood (i.e., before 18 years of age), indicating that environmental experiences have a harmful impact, especially during the critical developmental period of childhood to adolescence.

Our results suggest that the environment has a strong effect on the manifestation of violent behavior that may be rather independent of patients' mental condition. To investigate this hypothesis, we associated accumulated environmental risk and psychometrically collected aggression trait scores in two general population samples. Again, we found highly significant associations between risk and aggression traits. These findings emphasize not only the environment's influence on violent behavior, but also that our model of accumulated environmental risk can be used to predict aggressive behavior and identify individuals at risk.

Contrary to the large effects attributable to the environment, epigenetic analyses using epigenome-wide association scans remained behind our expectations; patients with different risk loads (low versus high) revealed comparable blood-derived DNA methylation levels. However, peripheral histone deacetylase 1 (HDAC1) mRNA levels, a gene encoding an important epigenetic modulator, were significantly higher in individuals who suffered from a high risk load. 


\subsection{Original publication}

Mitjans, M.*, Seidel, J.*, Begemann, M.*, Bockhop, F., Moya-Higueras, J., Bansal, V., Wesolowski, J., Seelbach, A., Ibáñez, M. I., Kovacevic, F., Duvar, O., Fañanás, L., Wolf, H.U., Ortet, G., Zwanzger, P., Klein, V., Lange, I., Tänzer, A., Dudeck, M., Penke, L., Tebartz van Elst, L., Bittner, R. A., Schmidmeier, R., Freese, R., Müller-Isberner, R., Wiltfang, J., Bliesener, T., Bonn, S., Poustka, L., Müller, J. L., Arias, B., Ehrenreich, H. (2018). Violent aggression predicted by multiple pre-adult environmental hits. Molecular Psychiatry, 24(10), 1549-1564.

${ }^{*}$ Equally contributing authors

\section{Personal contribution}

I was involved in the ongoing process of GRAS patient examinations, thoroughly assessing more than 100 patients suffering from schizophrenia or schizoaffective disorder, and collecting, besides others, detailed information about negative environmental factors experienced before adulthood and aggressive behavior. I updated and organized our phenotypical databases with information while additionally supervising students in the process of data entry. Together with my first author colleagues and under the supervision of Prof. Hannelore Ehrenreich I created variables to investigate both potential environmental risk factors and aggressive behavior of different intensity. I was responsible for the creation and the validation of the Violent Aggression Severity Score (VASS) and for the design of our model of accumulated risk factors of violent aggression. Furthermore, I conducted extensive statistical analyses of all the phenotypical data in addition to comparing $H D A C 1$ methylation levels in different risk accumulation groups. Moreover, I was responsible for creating Figure 1 as well as Tables 1-3 of the final manuscript. Together with my first author colleagues and Prof. Hannelore Ehrenreich I contributed to the Introduction, Methods, and Results sections of the manuscript and interpreted our findings for the Discussion section. Finally, I answered questions and requests from editors and reviewers during the process of submission and publication and read and corrected the final version of the manuscript. 


\title{
Violent aggression predicted by multiple pre-adult environmental hits
}

\author{
Marina Mitjans ${ }^{1,2,3} \cdot$ Jan Seidel $^{1} \cdot$ Martin Begemann ${ }^{1,4} \cdot$ Fabian Bockhop $^{1} \cdot$ Jorge Moya-Higueras $^{3,5}$. \\ Vikas Bansal $\mathbb{D}^{1,6}$ • Janina Wesolowski ${ }^{1}$. Anna Seelbach ${ }^{1}$ - Manuel Ignacio Ibáñez ${ }^{3,7}$ - Fatka Kovacevic ${ }^{1}$. \\ Oguzhan Duvar $^{1} \cdot$ Lourdes Fañanás $^{3,8} \cdot$ Hannah-Ulrike Wolf ${ }^{1} \cdot$ Generós Ortet $^{3,7} \cdot$ Peter Zwanzger $^{9} \cdot$ Verena Klein $^{10}$. \\ Ina Lange ${ }^{11} \cdot$ Andreas Tänzer $^{12} \cdot$ Manuela Dudeck $^{13} \cdot$ Lars Penke $^{14} \cdot$ Ludger Tebartz van Elst $^{15} \cdot$ Robert A. Bittner $^{16}$. \\ Richard Schmidmeier ${ }^{9} \cdot$ Roland Freese ${ }^{17} \cdot$ Rüdiger Müller-Isberner ${ }^{17}$ • Jens Wiltfang $\left(\mathbb{D}^{4} \cdot\right.$ Thomas Bliesener $^{18}$. \\ Stefan Bonn $\mathbb{1}^{2,6} \cdot$ Luise Poustka $^{19} \cdot$ Jürgen L. Müller ${ }^{4,20} \cdot$ Bárbara Arias $^{3,8} \cdot$ Hannelore Ehrenreich ${ }^{1,2}$
}

Received: 1 November 2017 / Revised: 19 February 2018 / Accepted: 21 February 2018 / Published online: 24 May 2018

(c) The Author(s) 2018. This article is published with open access

\begin{abstract}
Early exposure to negative environmental impact shapes individual behavior and potentially contributes to any mental disease. We reported previously that accumulated environmental risk markedly decreases age at schizophrenia onset. Follow-up of matched extreme group individuals ( $\leq 1$ vs. $\geq 3$ risks) unexpectedly revealed that high-risk subjects had $>5$ times greater probability of forensic hospitalization. In line with longstanding sociological theories, we hypothesized that risk accumulation before adulthood induces violent aggression and criminal conduct, independent of mental illness. We determined in 6 independent cohorts (4 schizophrenia and 2 general population samples) pre-adult risk exposure, comprising urbanicity, migration, physical and sexual abuse as primary, and cannabis or alcohol as secondary hits. All single hits by themselves were marginally associated with higher violent aggression. Most strikingly, however, their accumulation strongly predicted violent aggression (odds ratio 10.5). An epigenome-wide association scan to detect differential methylation of blood-derived DNA of selected extreme group individuals yielded overall negative results. Conversely, determination in peripheral blood mononuclear cells of histone-deacetylase1 mRNA as 'umbrella mediator' of epigenetic processes revealed an increase in the high-risk group, suggesting lasting epigenetic alterations. Together, we provide sound evidence of a disease-independent unfortunate relationship between well-defined pre-adult environmental hits and violent aggression, calling for more efficient prevention.
\end{abstract}

\section{Introduction}

Early exposure to external risk factors like childhood maltreatment, sexual abuse or head trauma, but also living in urban environment or migration from other countries and cultures, have long been known or suspected to exert adverse effects on individual development and

These authors contributed equally: Marina Mitjans, Jan Seidel, Martin Begemann.

\section{Bárbara Arias}

barbara.arias@ub.edu

$\triangle$ Hannelore Ehrenreich ehrenreich@em.mpg.de

Extended author information available on the last page of the article socioeconomic functioning. Moreover, these environmental risk factors seem to contribute to abnormal behavior and to severity and onset of mental illness [1-11], even though different risk factors may have different impact, dependent on the particular neuropsychiatric disease in focus. On top of these 'primary' factors, that are rather inevitable for the affected, 'secondary', avoidable risks add to the negative individual and societal outcome, namely cannabis and alcohol abuse [1, 11-16].

Adverse experiences in adulthood, like exposure to violence, traumatic brain injury, or substance intoxication, can act as single triggers to increase the short-term risk of violence in mentally ill individuals as much as in control subjects [16, 17]. However, comprehensive studies, including large numbers of individuals and replication cohorts, on pre-adult accumulation of environmental risk factors and their long-term consequences on human behavior do not exist. In a recent report, we showed that 
accumulation of environmental risks leads to a nearly 10year earlier schizophrenia onset, demonstrating the substantial impact of the environment on mental disease, which by far outlasted any common genetic effects [18]. To search for epigenetic signatures in blood of carefully matched extreme group subjects of this previous study (with $\leq 1$ vs. $\geq 3$ risk factors) we had to re-contact them. This re-contact led to the unforeseen observation that high-risk subjects had $>5$ times higher probability to be hospitalized in forensic units compared to low-risk subjects.

This finding stimulated the present work: Having the longstanding concepts of sociologists and criminologists in mind, we hypothesized that early accumulation of environmental risk factors would lead to increased violent aggression and social rule-breaking in affected individuals, independent of any mental illness. To test this hypothesis, we explored environmental risk before the age of 18 years in 4 schizophrenia samples of the GRAS (Göttingen Research Association for Schizophrenia) data collection [19, 20]. Likewise, risk factors were assessed as available in 2 general population samples. In all cohorts, accumulation of pre-adult environmental hits was highly significantly associated with lifetime conviction for violent acts or high psychopathy and aggressionhostility scores as proxies of violent aggression and rulebreaking. As a first small hint of epigenetic alterations in our high-risk subjects, histone-deacetylase1 (HDACl) mRNA was found increased in peripheral blood mononuclear cells (PBMC).

\section{Methods}

\section{Subjects}

\section{Schizophrenia}

Ethics Committees of Georg-August-University, Göttingen, and participating centers across Germany approved the GRAS study, complying with the Helsinki Declaration. All patients (and/or legal representatives) gave written informed consent. GRAS data collection-I (2005-2010) [19, 20] and -II (2013-2016) consist of schizophrenic and schizoaffective subjects, assigned to: (1) male discovery sample $(N=$ 134 extreme group individuals with $\leq 1$ or $\geq 3$ risk factors, selected/matched from our previous study [18]); (2) male GRAS-I $(N=606)$; (3) male GRAS-II $(N=320)$; (4) female GRAS-I and -II cohorts $(N=503)$.

\section{General population}

Replication samples IV $(N=336)$ and V $(N=229)$ consist of individuals from the Spanish general population, recruited from the Jaume I University in Castelló and drawn from the third wave of an ongoing follow-up study which recruited students from a variety of urban and rural, public and private high schools from Castelló. Ethical approval was obtained from University Ethics Committees; participants provided written informed consent $[21,22]$.

\section{Sociodemographic and disease-related parameters}

The GRAS data collection contains comprehensive information regarding sociodemographic and disease-related parameters, acquired through detailed examination, semistructured interviews, telephone consultations, questionnaires, and complete collection of hospitalization letters, allowing meticulous double-checking of patients' selfreports [19, 20]. Chlorpromazine equivalents as indicator of present medication/disease severity and past suicide attempts as measure of severe self-aggression were employed for sample characterization and group comparison. Premorbid intelligence was estimated using $M W T-B$ (Mehrfachwahl-Wortschatz-Intelligenztest-B), and for current cognitive symptoms, a cognitive composite score was calculated, based on reasoning (Leistungsprüfsystemsubtest-3), executive function (Trail-Making-B) and verbal learning and memory (VLMT) $[18,19]$.

\section{Environmental risk exposure}

\section{Schizophrenia subjects}

Specific information was derived from history-taking and semi-structured interviews with patients and relatives/caretakers (GRAS-Manual) [19, 20] and from SCID-I. Each patient was dichotomously classified as having/not having been exposed premorbid and until age 18 years to severe physical abuse (comprising unpredictability of violence, injury due to physical reprimand or objects for corporal punishment), sexual abuse (forced touches, kissing, attempted or real rape), migration (subjects immigrating to Germany), neurotrauma (traumatic brain injury of all severity grades), perinatal complications (pregnancy, delivery, early postnatal life), any cannabis consumption and alcohol abuse [23]. To operationalize urbanicity until age 18 , information on place of residence and relocation was collected from discharge letters, social history, telephone interviews/return mail (questionnaire). Total urbanicity score was dichotomously divided into rural vs. urban residence [18]. In case of contradictory or missing information, patients were excluded from respective analyses. Single risk factors with highest impact over all samples were accumulated to investigate combined influence on aggression. 


\section{General population subjects}

Physical and sexual abuse was assessed by the shortened version of Childhood Trauma Questionnaire (CTQ) [24] and dichotomously recorded (never/any), as was migration (not born in Spain), alcohol (Alcohol Use Disorders Identification Test - AUDIT $\geq 4$ ) [25] and any cannabis consumption. Data regarding perinatal complications, neurotrauma and urbanicity were unavailable.

\section{Measures of violent aggression and criminal conduct}

\section{Schizophrenia subjects}

History of forensic hospitalization or conviction for battery, sexual assault, manslaughter, murder (at least once in life time) was used as violent aggression proxy. For crossvalidation of this dichotomous variable, a continuous measure, the violent aggression severity score (VASS), based on questionnaires, interviews and charts, was generated and applied to the discovery sample. The VASS in turn was cross-validated by an intra-sample ranking of relative aggression severity by 2 independent raters (Fig. 1).

\section{General population subjects}

Secondary psychopathy of the Levenson Self-Report Psychopathy Scale (LSRP) [26], measuring antisocial aspects of psychopathy (rule-breaking; lack of effort towards socially rewarded behavior), and aggression-hostility factor of the Zuckerman-Kuhlman Personality Questionnaire, shortened form (ZKPQ-50-CC) [27], were used as proxies of violent aggression.

\section{Statistical analysis of environmental risk}

Group differences for continuous variables were assessed using Mann-Whitney- $U$ or Kruskal-Wallis- $H$ test for comparison of $>2$ groups. Frequency differences between groups were assessed using $\chi^{2}$-test or Fisher's exact test. As trend tests, Jonckheere-Terpstra or Cochran-Armitage tests were applied. Covariates are explained in display items. Bonferroni correction accounted for multiple testing ( $p$ values withstanding correction denoted). Statistical analyses were performed using SPSS (v17.0; IBM-Deutschland $\mathrm{GmbH}$, Munich, Germany), or R (v3.3.2; R-Foundation for Statistical Computing, Vienna, Austria).

\section{Methylation Array}

Whole blood-derived DNA of extreme group individuals $(N=134)$ was analyzed by Infinium-HumanMethylation $450 \mathrm{~K}$
(Illumina Inc, CA, USA). Raw intensity data was preprocessed and SWAN (Subset-quantile Within Array Normalization) performed using Bioconductor package Minfi (v1.18.6) [28]. Probes with annotated single-nucleotide polymorphisms (SNPs) in $\mathrm{CpG}$ site or at single base extension sites were removed, leaving 467,971 probes total. To identify differentially methylated positions, a linear regression model using limma (v3.28.17) Bioconductor package [29] was fit. Covariates were age, medication and estimated cell proportions (monocytes, granulocytes, CD4T, CD8T, natural killer, and B-cells), calculated using Cell Counts Function in Minfi package [30]. A total of $N=129$ individuals were finally included for the analyses since two samples were dropped based on separate clustering in principal component analysis and information regarding medication was not available for three samples. All analyses were performed in $\mathrm{R}$.

\section{PBMC isolation and HDAC1 assay}

PBMC were isolated from morning blood, collected into CPDA-vials (Citrate-Phosphate-Dextrose-Adenine, Sarstedt, Germany), applying standard Ficoll-Paque-Plus isolation (GE-Healthcare, Munich, Germany). Total RNA extraction was done using miRNeasy Mini-kit (Qiagen, Hilden, Germany). For reverse transcription, 200ng RNA was applied using a mixture of oligo(dT)/hexamers, dNTPs, DTT and 200U SuperscriptIII (Life Technologies GmbH, Darmstadt, Germany). HDACl expression was measured using quantitative real-time PCR. The cDNA was diluted 1:12.5 in $10 \mu \mathrm{l}$ reaction-mix, containing $5 \mu \mathrm{l}$ of SYBR-green (Life Technologies) and 1pmol/primer:

HDAC1-Fw: 5'-AAATTCTTGCGCTCCATCCG-3' HDACl-Rv: 5'-CAGGCCATCGAATACTGGACA-3' GAPDH-Fw: 5'-CTGACTTCAACAGCGACACC-3' GAPDH-Rv: 5'-TGCTGTAGCCAAATTCGTTGT-3'

Technical triplicates were run on LightCycler480 (Roche-Diagnostics GmbH, Mannheim, Germany). Relative HDACl expression was calculated by the thresholdcycle method (LightCycler480 Software1.5.0SP3-Roche) and normalization to the housekeeping gene $G A P D H$ was performed. After examination for outliers, Student's $t$ test was used to compare groups using Prism4 (GraphPadSoftware; San Diego, CA, USA).

\section{Results}

The environmental risk factors evaluated in this study comprise urbanicity, migration, perinatal complications, physical maltreatment, sexual abuse, traumatic brain injury, cannabis consumption and alcohol abuse. Contacting male extreme group subjects of GRAS (with low vs. high 


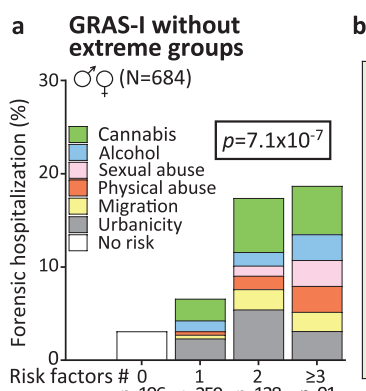

Risk factors $\underset{n=196}{\#} \underset{n=259}{1} \stackrel{1}{2} \underset{n=138}{2} \quad \underset{n=91}{\geq 3}$

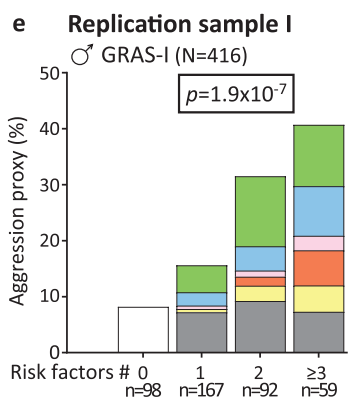

i Onset of aggressive behavior $\checkmark "$ Discovery sample $(\mathrm{N}=134)$

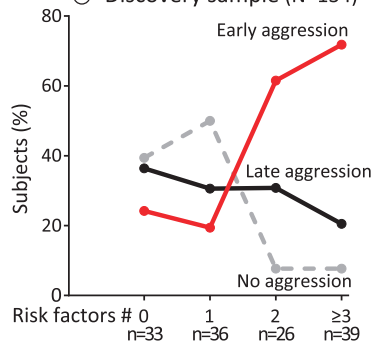

b Violent aggression severity score (VASS) calculation
\begin{tabular}{|lccc|}
\hline Type of aggression & Once & Twice & $>$ Twice \\
No documented aggression & 0 & 0 & 0 \\
Recurring aggressive thoughts/indirect threats & 1 & 1 & 1 \\
Deviant behavior & 1.5 & 3 & 4.5 \\
Criminality without aggression & 2 & 4 & 6 \\
Direct verbal aggression & 3 & 6 & 9 \\
Aggression against objects & 4 & 8 & 12 \\
Aggression against animals & 5 & 10 & 15 \\
Physical assaults without weapon: no injuries & 7 & 14 & 21 \\
Physical assaults without weapon: injuries & 10 & 20 & 30 \\
Rape & 12 & 24 & 36 \\
Physical assaults with weapon: no injuries & 12 & 24 & 36 \\
Physical assaults with weapon: injuries & 15 & 30 & 45 \\
Aggression with lethal consequence & 100 & 200 & 300 \\
\hline
\end{tabular}

j Replication IV-V

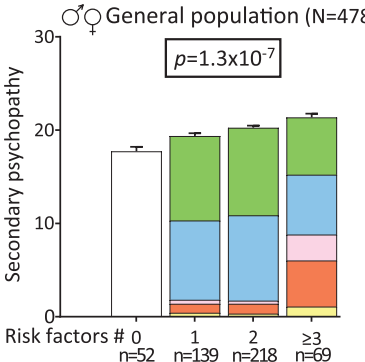

Fig. 1 Multiple environmental hits before adulthood predict violent aggression in mentally ill subjects as well as in the general population - Results from 6 independent samples. a Distribution of forensic hospitalization in the discovery sample (see results) suggested a substantial impact of environmental risk accumulation on violent aggression, a finding replicated in the remaining GRAS sample (GRAS-I males and females minus extreme group subjects of the discovery sample). Note the 'stair pattern' upon stepwise increase in risk factors; stacked-charts illustrate risk factor composition in the respective groups (including all risk factors of each individual in the respective risk group). Each color represents a particular risk (same legend for $\mathbf{d}-\mathbf{g}$ and $\mathbf{j}-\mathbf{k}) ; \chi^{2}$ test (two-sided). $\mathbf{b}$ Brief presentation of the violent aggression severity score, VASS, ranging from no documented aggression to lethal consequences of violent aggression with relative weight given to severity of aggression and number of registered reoccurrences. c Highly significant intercorrelation of violent aggression

environmental risk before age 18; discovery sample; $N=$ 134) [18] for a planned epigenetic follow-up, we found $27 \%$ of high-risk individuals in forensic units in contrast to only $6 \%$ of low-risk subjects ( $p<0.001 ; \chi^{2}$-test, two-sided). This finding was replicated in the remaining GRAS-I sample (GRAS-I males and females minus extreme group subjects), where a stepwise increase in lifetime prevalence of forensic hospitalization was seen upon risk accumulation (Fig. 1a).

This observation made us wonder whether we would find a strong intercorrelation between the here investigated

c Intercorrelation of aggression readouts
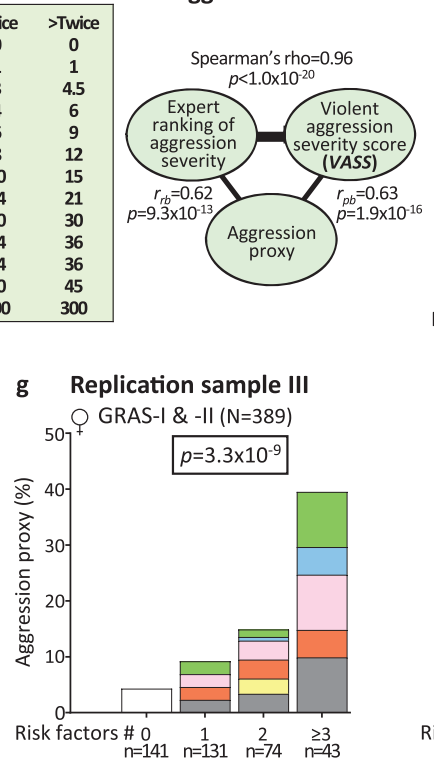

k Replication IV-V
O'OGeneral population $(\mathrm{N}=476)$

d Discovery sample

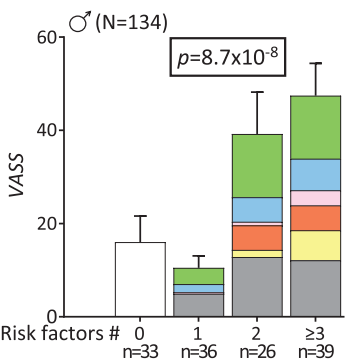

h Relative risk of aggression
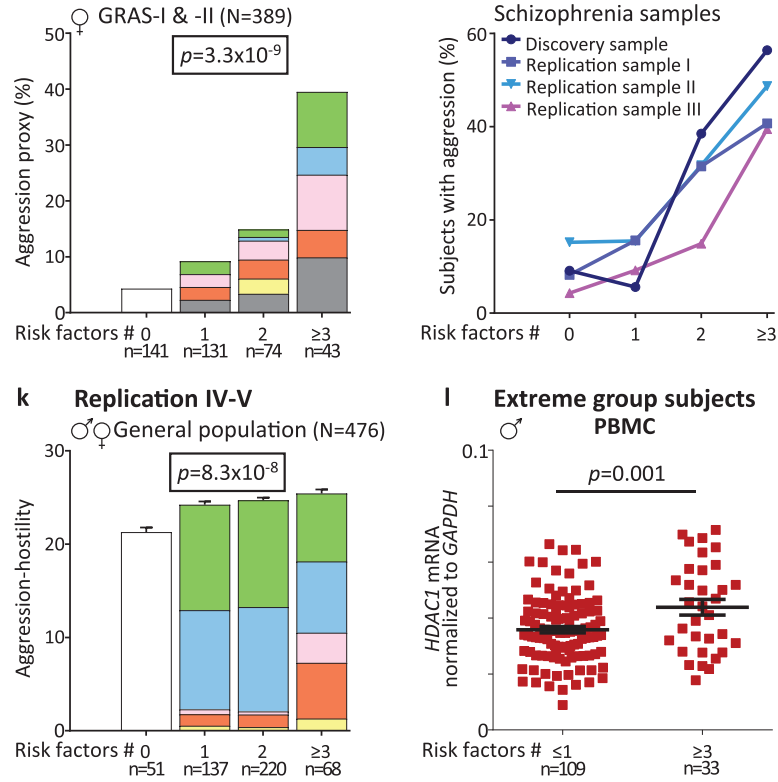

I Extreme group subjects

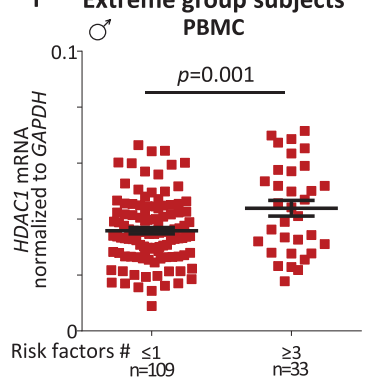

measures used in the present paper. d Application of VASS to risk accumulation in the discovery sample; Kruskal-Wallis- $H$ test (twosided). e-g Schizophrenia replication cohorts I-III: 'stair pattern' of aggression proxy in risk accumulation groups; all $\chi^{2}$ test (one-sided). $\mathbf{h}$ Comparative presentation of subjects $(\%)$ with violent aggression in risk accumulation groups across schizophrenia cohorts. i Comparative presentation of subjects (\%) with violent aggression before (premorbid, 'early') or after schizophrenia onset ('late') vs. individuals without evidence of aggression ('no') in risk accumulation groups of the discovery sample. $\mathbf{j}-\mathbf{k}$ General population replication cohorts IV and V: 'stair pattern' of aggression proxies, LSRP secondary psychopathy score (j) and aggression-hostility factor of $Z K P Q-50-C C(\mathbf{k})$ in risk accumulation groups; Kruskal-Wallis- $H$ test (one-sided). I HDACl mRNA levels in PBMC of male extreme group subjects as available for analysis; Student's $t$ test (one-sided)

environmental risks. To test for multicollinearity between the risk factors included in the accumulation models, we calculated the variance inflation factor (VIF) for each sample. Our results suggest that none of the included factors significantly collinears with any other (for each sample $\mathrm{VIF} \leq 1.28$ ), allowing us to include them in our models.

We hypothesized that forensic hospitalization reflects violent aggression. To quantify this trait, and in absence of established instruments for comprehensive retrospective analysis of violent aggression, we generated the VASS 
(Fig. 1b). Information for VASS was extracted for all discovery individuals $(N=134)$ from detailed history, available in the GRAS database [19, 20], and additional extensive chart study based on original medical documents over lifetime. VASS ranges from no documented aggression to lethal consequences of violent aggression. Relative weight is given to severity of aggression and number of registered re-occurrences. For first cross-validation of this new tool, an intra-sample expert ranking of relative aggression severity in the discovery sample was performed by 2 independent psychologists (unaware of environmental risk status of subjects under study), yielding Spearman's rho $=0.97$ for interrater reliability and rho $=0.96$ for intercorrelation with VASS (Fig. 1c). Inspection of VASS values in the discovery sample upon risk accumulation again demonstrates the 'stair pattern' (Fig. 1d).

Since not all information was available as detailed for the schizophrenia replication samples of GRAS-I and -II as for the discovery sample, we introduced a dichotomous aggression proxy, including history of forensic hospitalization and/or conviction for battery, sexual assault, manslaughter or murder (at least once in lifetime). Intercorrelation with VASS and expert ranking, respectively, resulted in $r_{p b}=0.63$ (point-biserial) and $r_{r b}=0.62$ (rankbiserial) (Fig. 1c). Applying this proxy to replication samples I-III (GRAS-I males without discovery sample, GRASII males, GRAS-I\&II females), consistently yielded the 'stair pattern' upon risk accumulation, even though at slightly lower level in females (Fig. 1e-g). The percentage of subjects with documented aggression increases with the number of risk factors, strikingly similar in all schizophrenia cohorts (Fig. 1h). Important for future preventive measures in at-risk subjects is the observation that a single risk factor (independent of its kind) is still compensated for (Fig. 1h). When comparing subjects with 0 vs. $\geq 3$ environmental factors over all schizophrenia samples, the odds ratio for violent aggression (based on aggression proxy) amounts to 10.5. Details on sociodemographic and diseaserelated variables, as well as on the various highly intercorrelating measures of violent aggression in the environmental risk accumulation groups in discovery and replication samples are given in Tables 1 and 2. Whereas no consistent differences in premorbid intelligence, present cognition (cognitive composite), and chlorpromazine equivalents (relative amount of antipsychotics) emerge among groups, age tends to be lower and suicidality to occur more frequently with increasing pre-adult environmental risk exposure in the schizophrenia cohorts, which is not unexpected considering our previous report [18] (Table 1). A remarkable increase in all available measures of violent aggression becomes obvious upon accumulation of environmental risk (final model consisting of urbanicity, migration, physical and sexual abuse, alcohol and cannabis), reflected by highly significant $p$ values in group and trend statistics throughout samples (Table 2).

For analyzing onset of aggressive behavior, the extensive information on aggression available in the discovery sample was exploited. Early aggression (any aggression documented before age 18 years and well before schizophrenia onset) clearly increased upon $\geq 2$ risk factors, whereas aggression seen only later in life seemed independent of early environmental risk (Fig. 1i). Therefore, we hypothesized that violent aggression upon risk accumulation may be unrelated to mental disease.

To test this hypothesis, we had the chance to analyze 2 well-characterized independent samples (replication IV and $\mathrm{V}$; Tables 1 and 2) of young individuals from the Spanish general population. Since data on criminal conduct could not be obtained in these cohorts, we had to use alternative, psychometrically validated instruments as aggression proxies, namely LSRP secondary psychopathy score [26], measuring rule-breaking and lack of effort towards socially rewarded behavior, and the aggression-hostility factor of $Z K P Q-50-C C$ [27]. Urbanicity as risk factor was unavailable in these samples (reducing the model to 5 of the 6 risk factors explored in schizophrenia, that is migration, physical maltreatment, sexual abuse, alcohol and cannabis). We also note that subjects were younger and as academics probably higher educated as compared to the disease cohorts. Despite these mitigating facts, and despite employing individuals of another country, the expected 'stair pattern' still emerged clearly for both proxies, likely suggesting generalizability of these findings (Fig. 1j,k; Tables 1 and 2). Data given here for the general population samples (replications IV and V) are based on both males and females. In addition, evaluating men and women separately (taking both general population cohorts together) yielded significant results for both genders (Table 2 bottom).

Addressing the composition of risk factors among groups across cohorts, we obtained a comparable pattern throughout schizophrenia samples (stacked-charts; Fig. 1a,d-g). In the general population subjects, particularly alcohol and cannabis consumption (classified as 'secondary hits') predominated (Fig. 1j,k) which also seem to play an appreciable role in schizophrenia cohorts. Therefore, we wondered whether separate analysis of risk accumulation, integrating only primary vs. only secondary hits, would still result in significant effects on aggression. For all schizophrenia samples individually, group difference and trend remained highly significant (not shown). Taking all schizophrenia subjects together $(N>1200)$, the aggression proxy yields for the accumulation model, built on primary risks only (urbanicity, migration, physical and sexual abuse), $p=$ $4.5 \times 10^{-17}\left(\chi^{2}=75.28\right)$ and $p<2.2 \times 10^{-16}\left(\chi^{2}=68.28\right)$, for group differences and trend, respectively. The corresponding results for secondary risk factors (alcohol, 
Table 1 Presentation of environmental risk groups in discovery and replication samples: sociodemographic and disease-related measures

\begin{tabular}{|c|c|c|c|c|c|}
\hline & No risk factors & 1 risk factor & 2 risk factors & $\geq 3$ risk factors & $p$ value $\left(H / \chi^{2}\right)$ \\
\hline \multicolumn{6}{|l|}{ Discovery sample $^{\text {a }}(N=121-134)$} \\
\hline Male schizophrenic subjects & $n=30-33$ & $n=32-36$ & $n=24-26$ & $n=35-39$ & \\
\hline Age (years) ${ }^{b}$ & $33.09(10.24)$ & $35.68(11.23)$ & $31.47(8.27)$ & $32.46(8.66)$ & $p=0.630(H=1.73)$ \\
\hline Premorbid intelligence $M W T-B^{\mathrm{c}}$ & $103.23(16.57)$ & $101.09(11.80)$ & $104.48(14.36)$ & $97.42(14.91)$ & $p=0.172(H=5.00)$ \\
\hline Cognitive composite score $^{\mathrm{d}}$ & $-0.05(1.13)$ & $-0.49(1.07)$ & $0.22(0.72)$ & $0.03(1.00)$ & $p=0.651(H=1.64)$ \\
\hline Chlorpromazine equivalents & $751.09(696.52)$ & $771.87(1227.51)$ & $674.28(508.49)$ & $648.83(569.38)$ & $p=0.769(H=1.13)$ \\
\hline Suicidality $^{\mathrm{e}}$ & $11(33.3 \%)$ & $8(23.5 \%)$ & $9(34.6 \%)$ & $14(36.8 \%)$ & $p=0.651\left(\chi^{2}=1.64\right)$ \\
\hline \multicolumn{6}{|l|}{ Replication sample I $(N=392-411)$} \\
\hline GRAS I male schizophrenic subjects & $n=91-98$ & $n=156-166$ & $n=91-92$ & $n=53-59$ & \\
\hline Age (years) ${ }^{b}$ & $46.94(12.26)$ & $39.65(12.50)$ & $34.51(10.18)$ & $32.85(8.38)$ & $\underline{p=1.6 \times 10^{-5}}(H=24.87)$ \\
\hline Premorbid intelligence $M W T-B^{\mathrm{c}}$ & 105.35 (17.09) & $103.32(15.87)$ & $101.00(14.26)$ & $99.23(15.10)$ & $p=0.085(H=6.61)$ \\
\hline Cognitive composite score $^{\mathrm{d}}$ & $0.15(1.12)$ & $0.75(1.01)$ & $0.10(0.93)$ & $-0.01(0.89)$ & $p=0.873(H=0.70)$ \\
\hline Chlorpromazine equivalents & $611.92(571.29)$ & $703.38(585.70)$ & $686.03(608.01)$ & $836.07(622.14)$ & $p=0.059(H=7.43)$ \\
\hline Suicidality $^{\mathrm{e}}$ & $23(24.2 \%)$ & $57(34.5 \%)$ & $33(36.6 \%)$ & $33(55.9 \%)$ & $\underline{p=0.001}\left(\chi^{2}=16.11\right)$ \\
\hline \multicolumn{6}{|l|}{ Replication sample II $(N=238-290)$} \\
\hline GRAS II male schizophrenic subjects & $n=36-46$ & $n=68-84$ & $n=67-82$ & $n=67-78$ & \\
\hline Age (years) ${ }^{\mathrm{b}}$ & $45.57(15.02)$ & $42.17(13.83)$ & $38.50(14.08)$ & $35.75(10.52)$ & $p=0.011(H=11.20)$ \\
\hline Premorbid intelligence $M W T-B^{c}$ & $98.09(14.46)$ & $102.29(16.03)$ & $100.48(13.36)$ & $96.39(9.29)$ & $p=0.184(H=4.84)$ \\
\hline Cognitive composite score $^{\mathrm{d}}$ & $-0.23(1.24)$ & $-0.08(1.03)$ & $-0.01(0.84)$ & $-0.08(0.96)$ & $p=0.816(H=0.94)$ \\
\hline Chlorpromazine equivalents & $629.15(513.31)$ & $747.35(629.02)$ & $689.31(717.18)$ & $713.84(532.66)$ & $p=0.629(H=1.74)$ \\
\hline Suicidality $^{\mathrm{e}}$ & $8(20.5 \%)$ & $12(14.6 \%)$ & $25(31.6 \%)$ & $25(34.2 \%)$ & $p=0.018\left(\chi^{2}=10.08\right)$ \\
\hline \multicolumn{6}{|l|}{ Replication sample III $(N=345-386)$} \\
\hline GRAS I-II female schizophrenic subjects & $n=125-140$ & $n=118-130$ & $n=65-71$ & $n=37-43$ & \\
\hline Age (years) ${ }^{b}$ & $43.44(11.75)$ & $46.67(12.90)$ & $40.84(12.71)$ & $36.53(11.31)$ & $\underline{p=0.003}(H=13.83)$ \\
\hline Premorbid intelligence $M W T-B^{c}$ & $103.53(14.19)$ & $104.04(14.28)$ & $102.96(15.84)$ & $99.10(15.41)$ & $p=0.147(H=5.37)$ \\
\hline Cognitive composite score $^{\mathrm{d}}$ & $0.03(0.96)$ & $0.09(0.99)$ & $0.24(0.99)$ & $-0.21(1.00)$ & $p=0.164(H=5.11)$ \\
\hline Chlorpromazine equivalents & $536.52(579.61)$ & $564.04(506.21)$ & $620.48(628.30)$ & $650.87(477.23)$ & $p=0.167(H=5.07)$ \\
\hline Suicidality $^{\mathrm{e}}$ & $45(33.6 \%)$ & $59(46.5 \%)$ & $33(46.5 \%)$ & $22(53.7 \%)$ & $p=0.052\left(\chi^{2}=7.72\right)$ \\
\hline \multicolumn{6}{|l|}{ Replication sample IV $(N=299)$} \\
\hline General population & $n=39$ & $n=83$ & $n=133$ & $n=44$ & \\
\hline Age (years) & $26.44(4.81)$ & $25.93(2.46)$ & $25.56(3.50)$ & $25.25(3.64)$ & $p=0.117(H=5.89)$ \\
\hline Gender, female/male ( $\%$ male) & $29 / 10(25.6 \%)$ & $51 / 32(38.6 \%)$ & $78 / 55(41.4 \%)$ & $18 / 26(59.1 \%)$ & \\
\hline \multicolumn{6}{|l|}{ Replication sample V $(N=177-183)$} \\
\hline General population & $n=13$ & $n=54-56$ & $n=86-89$ & $n=24-25$ & \\
\hline Age (years) & $20.54(0.88)$ & $20.63(0.98)$ & $20.85(1.12)$ & $20.88(1.15)$ & $p=0.696(H=1.44)$ \\
\hline Gender, female/male ( $\%$ male) & $7 / 6(46.2 \%)$ & $41 / 15(26.8 \%)$ & $56 / 33(37.1 \%)$ & $20 / 5(20.0 \%)$ & \\
\hline
\end{tabular}

Data are uncorrected means (SD) or n (\%); for statistical analysis, Kruskal-Wallis- $H, \chi^{2}$, or Fisher's exact test was used, all $p$ values two-sided; Bonferroni-corrected $p$ values $<0.01$ are considered significant and underlined; because of missing data, sample sizes vary;

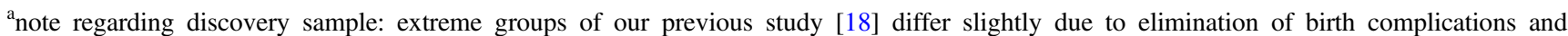
neurotrauma, but inclusion of alcohol in the present study;

${ }^{\mathrm{b}}$ corrected for age at disease onset;

${ }^{\mathrm{c}} M W T-B=$ Mehrfachwahl-Wortschatz-Intelligenztest-B;

${ }^{\mathrm{d}}$ cognitive composite score consists of reasoning (Leistungsprüfsystem-subtest-3), executive function (Trail-Making Test B), verbal learning \& memory test (VLMT) [18]; corrected for age, PANSS negative score, and chlorpromazine equivalents (standardized residuals after linear regression);

${ }^{\mathrm{e}}$ suicidality $=$ individuals with past suicide attempts 


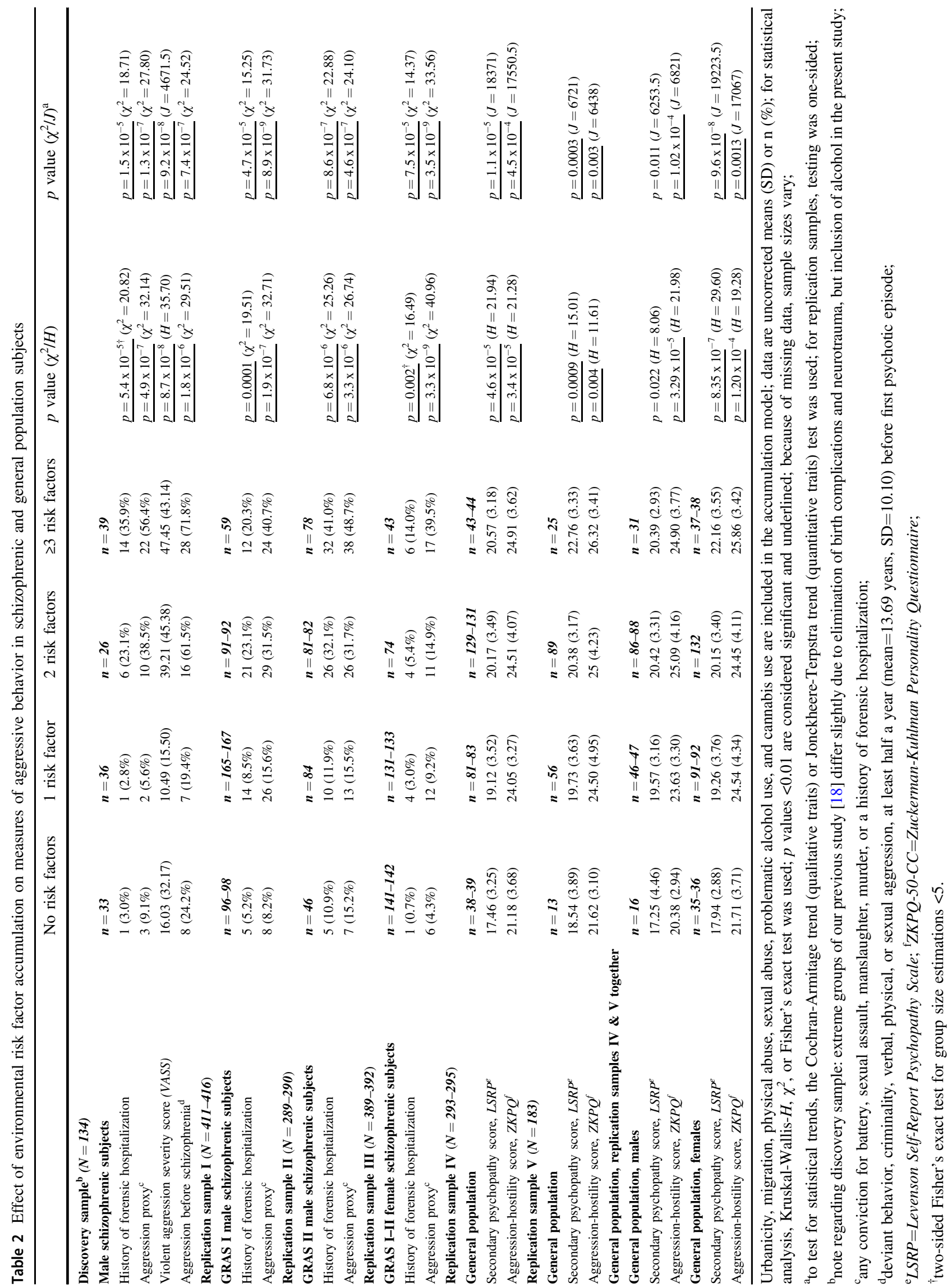


cannabis) in schizophrenia are $p=6.6 \times 10^{-19}\left(\chi^{2}=83.71\right)$ and $p<2.2 \times 10^{-16}\left(\chi^{2}=83.40\right)$. Analogously, taking all general population subjects together $(N>530)$, we obtain for $L S R P$ with primary risks (urbanicity not available) $p=$ $0.002(H=12.65)$ and $p=0.0003(J=33774.5)$, and with secondary risks $p=1.3 \times 10^{-4}(H=17.92)$ and $p=5.3 \times$ $10^{-5}(J=42412.5)$ for group differences and trend. Also here, significance was already reached with separate analysis of both cohorts (not shown).

For deciding on the accumulation model, we had initially screened all individual risk factors of our 'primary plus secondary risk factor model' separately in both schizophrenia and general population cohorts to get an estimation of their relative impact (Tables 3a,3b,3c). Perinatal complications and neurotrauma before the age of 18 years were unavailable for general population subjects. Since these risks showed the lowest overall impact on aggression proxies in schizophrenia, we decided not to include them in our present accumulation model.

Finally, we performed an epigenome-wide association scan to detect differential methylation of blood-derived DNA of selected extreme group individuals (discovery sample; $N=134$; Fig. 1a), originally planned as epigenetic follow-up study [18]. This scan turned out to be negative. In fact, contrasting subjects either with high vs. low number of environmental hits or according to VASS median split yielded a single methylation difference upon lowering the Bonferroni threshold to $10^{-6}$ (Table 4). Similarly, when looking in an exploratory fashion (small/unbalanced group sizes) at individual risk factors separately, results were essentially negative (Table 4). Hits associated with migration were likely related to ethnicity rather than environmental risk, as reported recently [31]. The power of our sample size - even though in the range of suggestions [32] and despite extreme group comparison - may not have been sufficient to detect differences, also due to a vast underlying heterogeneity of individual methylation sites. Even the search for methylation differences of aggression-related candidate genes [33-35] turned out negative (not shown), putting the relative weight of phenotypical consequences (here violent aggression) vs. common methylation results in humans into perspective. In contrast, determining $H D A C l$ mRNA levels in PBMC available from male extreme group subjects ( $\leq 1$ vs. $\geq 3$ risks) revealed a highly significant difference $(p=0.001)$, with higher levels in the high-risk $(N$ $=33$ ) compared to the low-risk group $(N=109)$ (Fig. 11). This transcript encodes an enzyme of the histone deacetylase complex which serves as an overarching regulator of epigenetic processes. Indeed, peripheral HDACl mRNA levels seem to be a more robust readout of epigenetic modifications in small sample sizes [36] as compared to specific methylation sites in the epigenome-wide association scan, and suggest lasting epigenetic alterations.

\section{Discussion}

The present work was initiated based on the observation in a schizophrenia cohort that accumulation of environmental risk factors before adulthood promotes the likelihood of later forensic hospitalization, interpreted as indicator of violent aggression. This interpretation and the effect of risk accumulation were consolidated using direct scoring of aggression over lifetime or, as aggression proxies, forensic hospitalization and conviction for battery, sexual assault, manslaughter or murder, or respective psychopathology measures in 4 independent schizophrenia cohorts and 2 general population samples. Importantly, our data support the concept of a disease-independent development of violent aggression in subjects exposed to multiple pre-adult environmental risk factors.

Whereas a vast amount of literature on single environmental risk factors reports consequences for abnormal behavior and mental illness, publications on pre-adult risk accumulation are scarce and mostly based on closely interrelated social/familial risk factors. Also, risk and consequence are often not clearly defined. Studies including larger, comprehensively characterized datasets and replication samples do not exist. The present work is the first to provide sound evidence, based on 6 separate cohorts, of a disease-independent relationship between accumulation of multifaceted pre-adult environmental hits and violent aggression. The overall societal damage is enormous, and we note that mentally ill individuals who re-enter the community from prison are even more at risk for unemployment, homelessness, and criminal recidivism [37]. These results should encourage better precautionary measures, including intensified research on protective factors which is still underrepresented [2, 38-40].

In the psychosociological literature, the so-called externalizing behavior in childhood includes hostile and aggressive physical behavior toward others, impulsivity, hyperactivity, and noncompliance with limit-setting $[41,42]$. The respective risk factors are all highly plausible, yet often theoretical, and derived from 4 broad domains: child risk factors (e.g., adverse temperament, genetic and gender risk), sociocultural risks (e.g., poverty, stressful life events), parenting and caregiving (e.g., conflict and violence at home, physical abuse), and children's peer experiences (e.g., instable relationships, social rejection). A full model of the development of conduct problems has been suggested to include at least these 4 domains [41, 43, 44]. The risk factors analyzed in the present study are perhaps somewhat clearer defined but partially related to and overlapping across these domains. Urbanicity, migration, cannabis and alcohol reflect sociocultural input but also peer experience, and physical or sexual abuse belong to the parenting/caregiver aspect. 


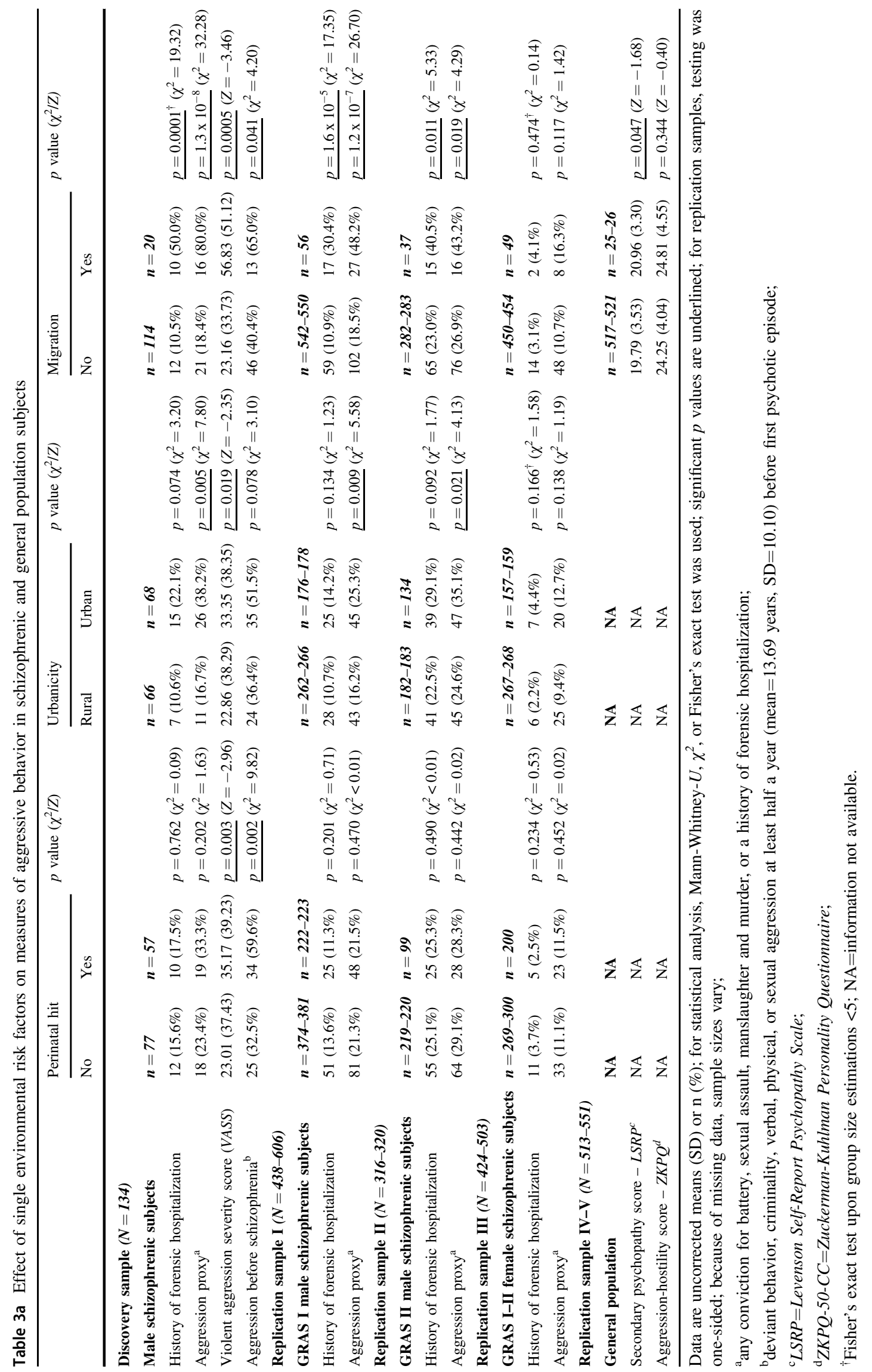




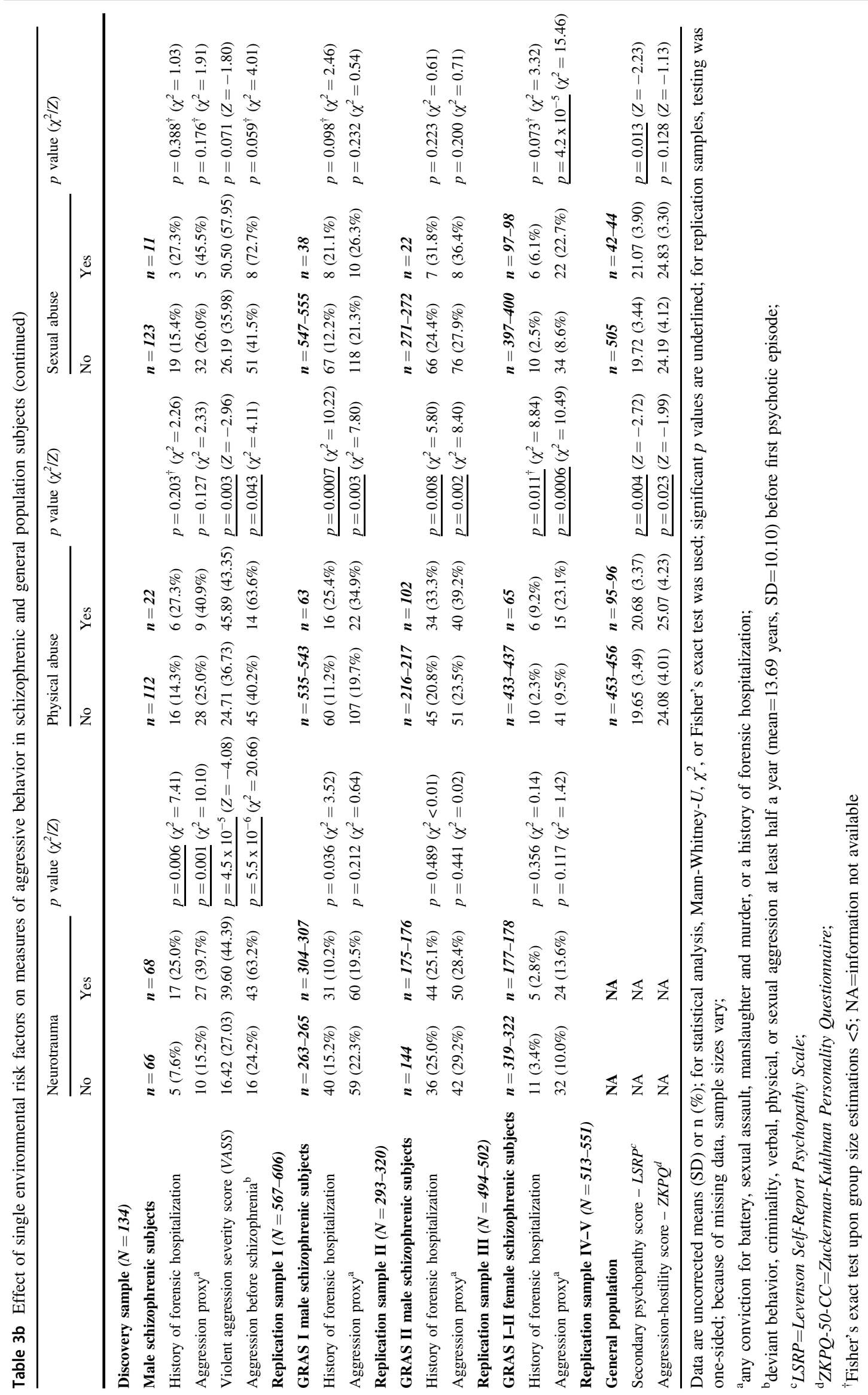




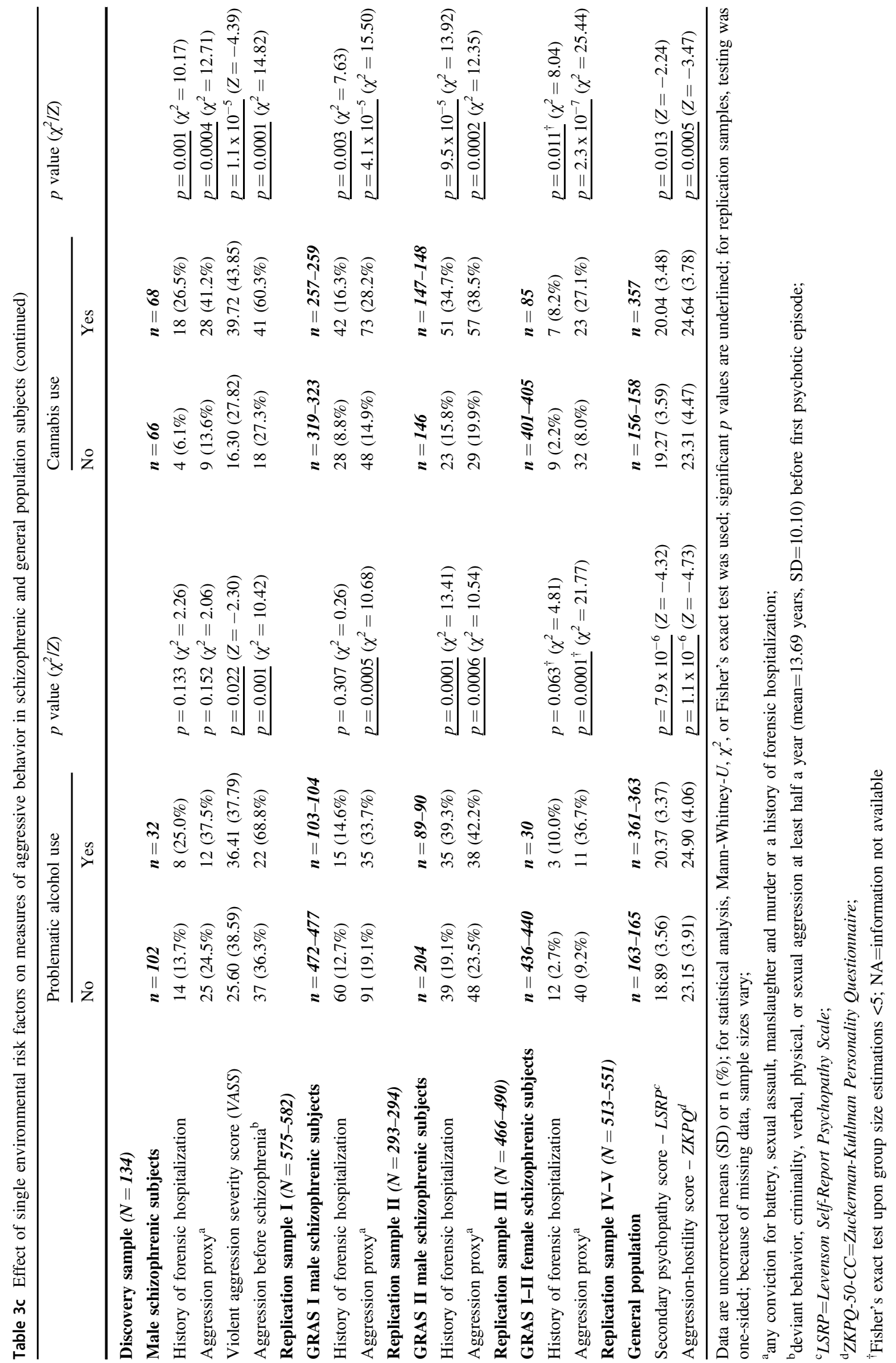




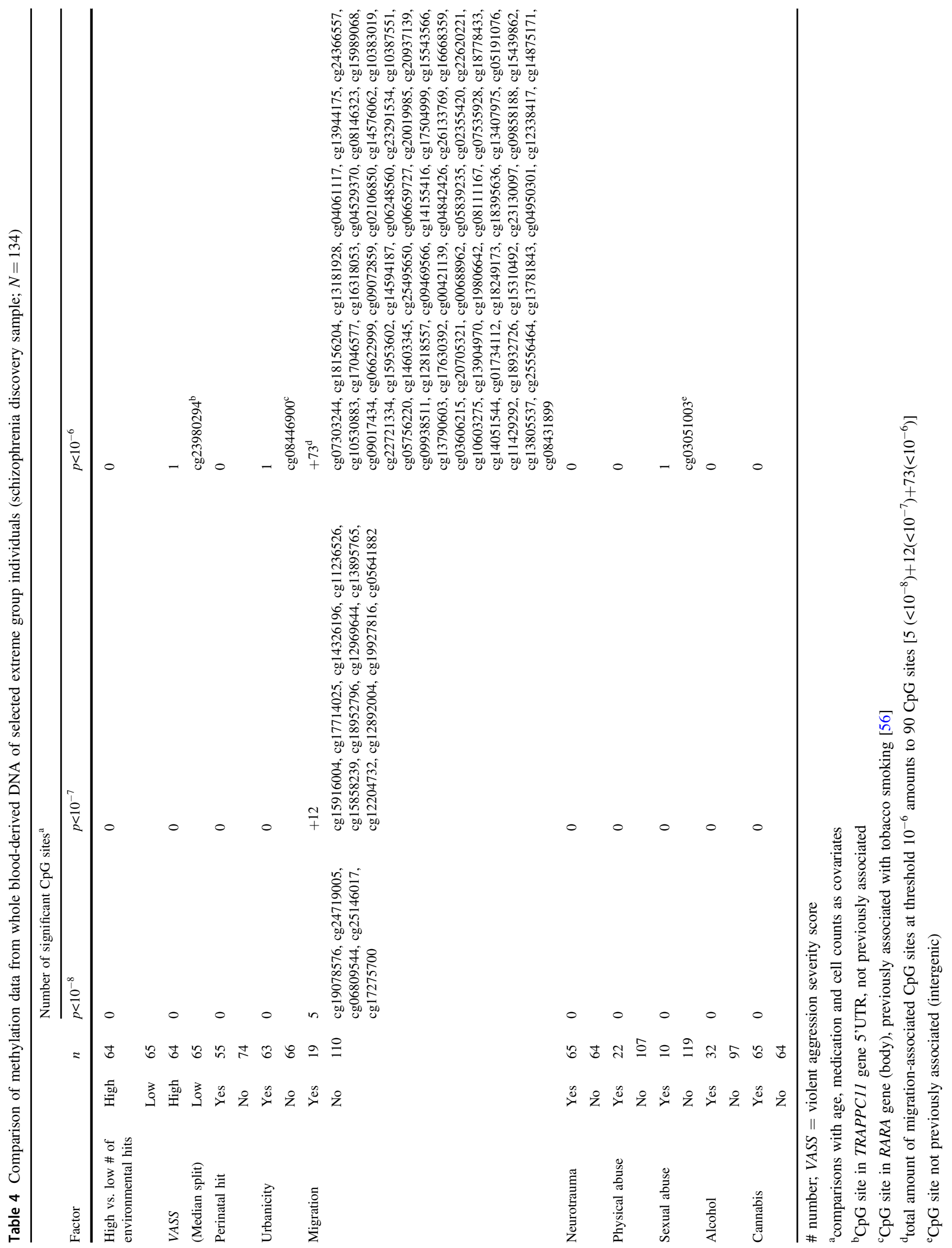


Certainly, there are many more, still undiscovered risk and numerous protective factors, potentially explaining why 'only' $40-50 \%$ of high-risk individuals in our schizophrenia samples fulfill criteria of violent aggression. We note that this study does not include genetic data analysis or correction for any genetic impact. The genetic influence on aggression, however, may be of considerable relevance for the individual [45-49], even though highly heterogeneous as for essentially all behavioral traits. Heritability of aggression, estimated from twin studies, reaches $>60 \%$ $[50,51]$. In fact, $50 \%$ of individuals with violent aggression upon pre-adult risk accumulation in the present study means another $50 \%$ without detectable aggression. This consistent finding across samples likely indicates that genetic predisposition is prerequisite for whichever behavioral consequence. Individuals without genetic predisposition and/or with more protective factors (genetic and environmental) may not react with violent aggression to accumulated environmental risk. Importantly, the obvious gender effect may be a matter of degree rather than of pattern. In fact, the etiology of externalizing behavior problems is similar for girls and boys [41, 52], as is the consequence of risk accumulation in the present study for males and females.

The risk factors of the sociological domains seem to be stable predictors over time, to some degree interchangeable, pointing to many pathways leading to the same outcome (principle of equifinality) [41]. The interchangeability is highly interesting also with respect to potential biological mechanisms. It appears that any of the here investigated hits alone, independent of its kind, can be compensated for but that higher risk load increases the probability of violent aggression. Also for that reason, we are weighing risk fators equally in the present study. This could theoretically create some bias. However, to be able to estimate the true effect size of each specific factor separately on violent aggression and subsequently weigh all factors in a more proper way, much larger samples sizes would be needed that are presently not available anywhere in the world.

In contrast to the marginal influence of genome-wide association data on mental disease in GRAS [18, 53], the accumulated environmental impact on development of violent aggression is huge, reflected by odds ratios of $>10$. When striking at a vulnerable time of brain development, namely around/before puberty, the environmental input may 'non-specifically' affect any predisposed individual. The hypothetical biological mechanisms underlying this accumulation effect in humans may range from alterations in neuroendocrine and neurotransmitter systems, neuronal/ synaptic plasticity and neurogenesis to changes in the adaptive immune system and interference with developmental myelination, affecting brain connectivity and network function $[9,10,54,55]$.
Our approach to detect methylation changes in blood using an epigenome-wide association scan was unsuccessful despite matched extreme group comparison, likely due to the small sample size (although in the suggested range [32]), and perhaps the etiological/pathogenetic complexity of accumulated risks. Changes in brain, not accessible here for analysis, can certainly not be excluded. Interestingly, however, HDACl mRNA levels in PBMC of male extreme group subjects were increased in the high-risk compared to the low-risk group. This finding confirms peripheral HDAC1 mRNA levels as a more robust readout of epigenetic alterations in relatively small sample sizes [36], as compared to specific methylation sites in epigenome-wide association scans or even in candidate genes. To gain further mechanistic insight and thereby develop - in addition to prevention measures - novel individualized treatment concepts [36], animal studies modeling risk accumulation seem unavoidable.

To conclude, this study should motivate sociopolitical actions, aiming at identifying individuals-at-risk and improving precautionary measures. Effective violence prevention strategies start early and include family-focused and school-based programs [2, 16, 38]. Additional risk factors, interchangeable in their long-term consequences, like urbanicity, migration, and substance abuse, should be increasingly considered. Health care providers are essential for all of these prevention concepts. More research on protective factors and resilience should be launched. Animal studies need to be supported that model risk accumulation for mechanistic insight into brain alterations leading to aggression, and for developing new treatment approaches, also those targeting reversal of epigenetic alterations. As a novel concept, scientific efforts on 'phenotyping of the environment' [11] should be promoted to achieve more fundamental risk estimation and more effective prevention in the future.

Acknowledgements This work was supported by the Max Planck Society, the Max Planck Förderstiftung, the DFG (CNMPB), EXTRABRAIN EU-FP7, the Niedersachsen-Research Network on Neuroinfectiology (N-RENNT), and EU-AIMS. The research of EUAIMS receives support from the Innovative Medicines Initiative Joint Undertaking under grant agreement $n^{\circ} 115300$, resources of which are composed of financial contribution from the European Union's Seventh Framework Programme (FP7/2007-2013), from the EFPIA companies, and from Autism Speaks. Moreover, support from Centro de Investigación en Red de Salud Mental (CIBERSAM); Instituto de Salud Carlos III (PI16/00998), Comissionat per a Universitats i Recerca del DIUE (2014SGR1636) and Spanish Ministry of Economy and Competitiveness (ES-EUEpiBrain, SAF2015-71526-REDT) is gratefully acknowledged. We thank all subjects for participating in the study, and all the many colleagues who have contributed over the past decade to the GRAS data collection.

Author Contributions: Concept, design and supervision of the study: HE, MM, JS. Data acquisition/analysis/interpretation: MB, JS, MM, 
FB, JMH, VB, JWE, AS, MII, FK, OD, LF, HW, GO, PZ, VK, IL, AT, MD, LP, LTVE, RAB, RS, RF, RMI, JW, TB, SB, LUP, JLM, BA, HE. Drafting manuscript and figures: HE, JS, MM, BA. All authors read and approved the final version of the manuscript.

\section{Compliance with ethical standards}

Conflict of interest The authors declare that they have no conflict of interest.

Open Access This article is licensed under a Creative Commons Attribution 4.0 International License, which permits use, sharing, adaptation, distribution and reproduction in any medium or format, as long as you give appropriate credit to the original author(s) and the source, provide a link to the Creative Commons license, and indicate if changes were made. The images or other third party material in this article are included in the article's Creative Commons license, unless indicated otherwise in a credit line to the material. If material is not included in the article's Creative Commons license and your intended use is not permitted by statutory regulation or exceeds the permitted use, you will need to obtain permission directly from the copyright holder. To view a copy of this license, visit http://creativecommons. org/licenses/by/4.0/.

\section{References}

1. McDonald C, Murray RM. Early and late environmental risk factors for schizophrenia. Brain Res Rev. 2000;31:130-7.

2. Raine A. Biosocial studies of antisocial and violent behavior in children and adults: a review. J Abnorm Child Psychol. 2002;30:311-26.

3. Read J, van Os J, Morrison AP, Ross CA. Childhood trauma, psychosis and schizophrenia: a literature review with theoretical and clinical implications. Acta Psychiatr Scand. 2005;112: $330-50$.

4. van Os J, Kenis G, Rutten BPF. The environment and schizophrenia. Nature. 2010;468:203-12.

5. Brown AS. The environment and susceptibility to schizophrenia. Prog Neurobiol. 2011;93:23-58.

6. Lederbogen F, Kirsch P, Haddad L, Streit F, Tost H, Schuch P, et al. City living and urban upbringing affect neural social stress processing in humans. Nature. 2011;474:498-501.

7. Wortzel HS, Arciniegas DB. A forensic neuropsychiatric approach to traumatic brain injury, aggression, and suicide. J Am Acad Psychiatry. 2013;41:274-86.

8. Orlovska S, Pedersen MS, Benros ME, Mortensen PB, Agerbo E, Nordentoft M. Head injury as risk factor for psychiatric disorders: A nationwide register-based follow-up study of 113,906 persons with headinjury. Am J Psychiat. 2014;171:463-9.

9. McEwen BS, Nasca C, Gray JD. Stress effects on neuronal structure: hippocampus, amygdala, and prefrontal cortex. Neuropsychopharmacology. 2016;41:3-23.

10. Nemeroff CB. Paradise lost: the neurobiological and clinical consequences of child abuse and neglect. Neuron. 2016;89: 892-909.

11. Ehrenreich $\mathrm{H}$. The impact of environment on abnormal behavior and mental disease. EMBO Rep. 2017;18:661-5.e201744197-n/a.

12. Giancola PR. Alcohol-related aggression during the college years: Theories, risk factors and policy implications. J Stud Alcohol. 2002;14:129-39.

13. Heinz AJ, Beck A, Meyer-Lindenberg A, Sterzer P, Heinz A. Cognitive and neurobiological mechanisms of alcohol-related aggression. Nat Rev Neurosci. 2011;12:400-13.
14. Large M, Sharma S, Compton MT, Slade T, Nielssen O. Cannabis use and earlier onset of psychosis. Arch Gen Psychiat. 2011;68:555-61.

15. Walsh E, Gilvarry C, Samele C, Harvey K, Manley C, Tattan T, et al. Predicting violence in schizophrenia: a prospective study. Schizophr Res. 2004;67:247-52.

16. Fazel S, Gulati G, Linsell L, Geddes JR, Grann M. Schizophrenia and violence: systematic review and meta-analysis. PLoS Med. 2009;6:e1000120.

17. Sariaslan A, Lichtenstein P, Larsson H, Fazel S. Triggers for violent criminality in patients with psychotic disorders. JAMA Psychiatry. 2016;73:796-803.

18. Stepniak B, Papiol S, Hammer C, Ramin A, Everts S, Hennig L, et al. Accumulated environmental risk determining age at schizophrenia onset: a deep phenotyping-based study. Lancet Psychiatry. 2014;1:444-53.

19. Begemann M, Grube S, Papiol S, Malzahn D, Krampe H, Ribbe $\mathrm{K}$, et al. Modification of cognitive performance in schizophrenia by complexin 2 gene polymorphisms. Arch Gen Psychiat. 2010;67:879-88.

20. Ribbe K, Friedrichs H, Begemann M, Grube S, Papiol S, Kastner A, et al. The cross-sectional GRAS sample: a comprehensive phenotypical data collection of schizophrenic patients. BMC Psychiatry. 2010;10:91.

21. Alemany S, Moya J, Ibanez MI, Villa H, Mezquita L, Ortet G, et al. Research Letter: Childhood trauma and the rs1360780 SNP of FKBP5 gene in psychosis: a replication in two general population samples. Psychol Med. 2016;46:221-3.

22. Ortet G, Ibanez MI, Moya J, Villa H, Viruela A, Mezquita L. Assessing the five factors of personality in adolescents: the junior version of the Spanish NEO-PI-R. Assessment. 2012;19:114-30.

23. Ribbe K, Ackermann V, Schwitulla J, Begemann M, Papiol S, Grube S, et al. Prediction of the risk of comorbid alcoholism in schizophrenia by interaction of common genetic variants in the corticotropin-releasing factor system. Arch Gen Psychiat. 2011;68:1247-56.

24. Bernstein DP, Stein JA, Newcomb MD, Walker E, Pogge D, Ahluvalia $\mathrm{T}$, et al. Development and validation of a brief screening version of the Childhood Trauma Questionnaire. Child Abus Negl. 2003;27:169-90.

25. Babor T, DeLaFuentes J, Saunders J, Grant M. The alcohol use disorders identification test: guidelines for use in primary health care. Vol. PSA. World Health Organization; Geneva, Switzerland, 1992. pp. 1-30.

26. Levenson MR, Kiehl KA, Fitzpatrick CM. Assessing psychopathic attributes in a noninstitutionalized population. J Pers Soc Psychol. 1995;68:151-8.

27. Aluja A, Rossier J, Garcia LF, Angleitner A, Kuhlman M, Zuckerman M. A cross-cultural shortened form of the ZKPQ (ZKPQ-50-cc) adapted to English, French, German, and Spanish languages. Pers Indiv Differ. 2006;41:619-28.

28. Aryee MJ, Jaffe AE, Corrada-Bravo H, Ladd-Acosta C, Feinberg AP, Hansen KD, et al. Minfi: a flexible and comprehensive Bioconductor package for the analysis of Infinium DNA methylation microarrays. Bioinformatics. 2014;30:1363-9.

29. Ritchie ME, Phipson B, Wu D, Hu Y, Law CW, Shi W, et al. limma powers differential expression analyses for RNAsequencing and microarray studies. Nucleic Acids Res. 2015;43: e47.

30. Jaffe AE, Irizarry RA. Accounting for cellular heterogeneity is critical in epigenome-wide association studies. Genome Biol. 2014; 15:R31.

31. Galanter JM, Gignoux CR, Oh SS, Torgerson D, Pino-Yanes M, Thakur N, et al. Differential methylation between ethnic subgroups reflects the effect of genetic ancestry and environmental exposures. Elife. 2017;6:e20532. 
32. Tsai PC, Bell JT. Power and sample size estimation for epigenome-wide association scans to detect differential DNA methylation. Int J Epidemiol. 2015;44:1429-41.

33. Waltes R, Chiocchetti AG, Freitag CM. The neurobiological basis of human aggression: a review on genetic and epigenetic mechanisms. Am J Med Genet B Neuropsychiatr Genet. 2016;171:650-75.

34. Gunter TD, Vaughn MG, Philibert RA. Behavioral genetics in antisocial spectrum disorders and psychopathy: a review of the recent literature. Behav Sci Law. 2010;28:148-73.

35. Kolla NJ, Matthews B, Wilson AA, Houle S, Bagby RM, Links P, et al. Lower monoamine oxidase-A total distribution volume in impulsive and violent male offenders with antisocial personality disorder and high psychopathic traits: an [(11)C] harmine positron emission tomography study. Neuropsychopharmacology. 2015;40:2596-603.

36. Bahari-Javan S, Varbanov H, Halder R, Benito E, Kaurani L, Burkhardt S, et al. HDAC1 links early life stress to schizophrenialike phenotypes. Proc Natl Acad Sci USA. 2017;114:E4686-94.

37. Swanson J. Mental illness, release from prison, and social context. JAMA. 2016;316:1771-2.

38. Sumner SA, Mercy JA, Dahlberg LL, Hillis SD, Klevens J, Houry D. Violence in the United States: status, challenges, and opportunities. JAMA. 2015;314:478-88.

39. Stoddard SA, Whiteside L, Zimmerman MA, Cunningham RM, Chermack ST, Walton MA. The relationship between cumulative risk and promotive factors and violent behavior among urban adolescents. Am J Commun Psychol. 2013;51: 57-65.

40. van der Laan AM, Veenstra R, Bogaerts S, Verhulst FC, Ormel J. Serious, minor, and non-delinquents in early adolescence: the impact of cumulative risk and promotive factors. The TRAILS Study. J Abnorm Child Psychol. 2010;38:339-51.

41. Deater-Deckard K, Dodge KA, Bates JE, Pettit GS. Multiple risk factors in the development of externalizing behavior problems: group and individual differences. Dev Psychopathol. 1998;10:469-93.

42. Mcmahon RJ. Diagnosis, assessment, and treatment of externalizing problems in children - the role of longitudinal data. $\mathbf{J}$ Consult Clin Psych. 1994;62:901-17.
43. Herrenkohl TI, Maguin E, Hill KG, Hawkins JD, Abbott RD, Catalano RF. Developmental risk factors for youth violence. J Adolesc Health. 2000;26:176-86.

44. Loeber R, Pardini D, Homish DL, Wei EH, Crawford AM, Farrington DP, et al. The prediction of violence and homicide in young men. J Consult Clin Psych. 2005;73:1074-88.

45. Mason DA, Frick PJ. The heritability of antisocial-behavior - a metaanalysis of twin and adoption studies. J Psychopathol Behav Assess. 1994;16:301-23.

46. Mednick SA, Gabrielli WF Jr, Hutchings B. Genetic influences in criminal convictions: evidence from an adoption cohort. Science. 1984;224:891-4.

47. Coccaro EF, Bergeman CS, Kavoussi RJ, Seroczynski AD. Heritability of aggression and irritability: a twin study of the BussDurkee aggression scales in adult male subjects. Biol Psychiatry. 1997;41:273-84.

48. Miles DR, Carey G. Genetic and environmental architecture of human aggression. J Pers Soc Psychol. 1997;72:207-17.

49. Rhee SH, Waldman ID. Genetic and environmental influences on antisocial behavior: a meta-analysis of twin and adoption studies. Psychol Bull. 2002;128:490-529.

50. DiLalla LF, Gottesman II. Biological and genetic contributors to violence--Widom's untold tale. Psychol Bull. 1991;109:125-9. discussion130-122

51. Plomin R, DeFries JC, Knopik VS, Neiderhiser J. Behavioral genetics. 6th ed. New York, NY: Worth Publishers; 2013.

52. Zoccolillo M. Gender and the development of conduct disorder. Dev Psychopathol. 1993;5:65-78.

53. Ehrenreich H, Mitjans M, Van der Auwera S, Centeno TP, Begemann M, Grabe HJ, et al. OTTO: a new strategy to extract mental disease-relevant combinations of GWAS hits from individuals. Mol Psychiatry. 2016;23:476-86.

54. Korosi A, Naninck EFG, Oomen CA, Schouten M, Krugers H, Fitzsimons C, et al. Early-life stress mediated modulation of adult neurogenesis and behavior. Behav Brain Res. 2012;227:400-9.

55. Nave KA, Ehrenreich H. Myelination and oligodendrocyte functions in psychiatric diseases. JAMA Psychiatry. 2014;71:582-4.

56. Su D, Wang X, Campbell MR, Porter DK, Pittman GS, Bennett $\mathrm{BD}$, et al. Distinct epigenetic effects of tobacco smoking in whole blood and among leukocyte subtypes. PLoS ONE. 2016;11: e0166486.

\section{Affiliations}

Marina Mitjans $s^{1,2,3} \cdot$ Jan Seidel $^{1} \cdot$ Martin Begemann ${ }^{1,4} \cdot$ Fabian Bockhop $^{1} \cdot$ Jorge Moya-Higueras $^{3,5}$. Vikas Bansal $\mathbb{1}^{1,6}$ - Janina Wesolowski ${ }^{1}$ Anna Seelbach ${ }^{1}$ - Manuel Ignacio Ibáñez ${ }^{3,7}$ - Fatka Kovacevic ${ }^{1}$. Oguzhan Duvar $^{1} \cdot$ Lourdes Fañanás $^{3,8} \cdot$ Hannah-Ulrike Wolf ${ }^{1} \cdot$ Generós Ortet $^{3,7} \cdot$ Peter Zwanzger $^{9} \cdot$ Verena Klein $^{10}$. Ina Lange ${ }^{11} \cdot$ Andreas Tänzer $^{12} \cdot$ Manuela Dudeck ${ }^{13} \cdot$ Lars Penke $^{14} \cdot$ Ludger Tebartz van Elst $^{15} \cdot$ Robert A. Bittner $^{16}$. Richard Schmidmeier $^{9} \cdot$ Roland Freese $^{17} \cdot$ Rüdiger Müller-Isberner ${ }^{17}$. Jens Wiltfang $\mathbb{1}^{4} \cdot$ Thomas Bliesener $^{18}$. Stefan Bonn $\mathbb{D}^{2,6} \cdot$ Luise Poustka ${ }^{19} \cdot$ Jürgen L. Müller ${ }^{4,20} \cdot$ Bárbara Arias $^{3,8} \cdot$ Hannelore Ehrenreich ${ }^{1,2}$

1 Clinical Neuroscience, Max Planck Institute of Experimental Medicine, Göttingen, Germany

2 DFG Research Center for Nanoscale Microscopy and Molecular Physiology of the Brain (CNMPB), Göttingen, Germany

3 Instituto de Salud Carlos III, Centro de Investigación Biomédica en Red de Salud Mental (CIBERSAM), Madrid, Spain

4 Department of Psychiatry \& Psychotherapy, University of Göttingen, Göttingen, Germany
5 Department of Psychology, Faculty of Education, Psychology and Social Work, University of Lleida, Lleida, Spain

6 Center for Molecular Neurobiology, Institute of Medical Systems Biology, University Clinic Hamburg-Eppendorf, Hamburg, Germany

7 Department of Basic and Clinical Psychology and Psychobiology, Universitat Jaume I, Castelló, Spain 
8 Departament Biologia Evolutiva, Ecologia i Ciències Ambientals, Facultat de Biologia and Institut de Biomedicina (IBUB),

Universitat de Barcelona, Barcelona, Spain

9 KBO-Inn-Salzach-Klinikum, Gabersee, Wasserburg am Inn, Germany

10 KBO-Isar-Amper-Klinikum, Taufkirchen (Vils), Germany

11 Competence Center for Forensic Psychiatry, Lower Saxony, MRV Moringen, Germany

12 Department of Forensic Psychiatry \& Psychotherapy, KRH, Wunstorf, Germany

13 Forensic Psychiatry and Psychotherapy, University of Ulm, Ulm, Germany

14 Institute of Psychology, University of Göttingen,
Göttingen, Germany

15 Department of Psychiatry \& Psychotherapy, University of Freiburg, Freiburg, Germany

16 Department of Psychiatry \& Psychotherapy, University of Frankfurt, Frankfurt, Germany

17 Vitos Forensic Psychiatric Hospital, Haina, Germany

18 Criminological Research Institute of Lower Saxony, Hannover, Germany

19 Department of Child and Adolescent Psychiatry \& Psychotherapy, University of Göttingen, Göttingen, Germany

20 Asklepios Hospital for Forensic Psychiatry \& Psychotherapy, Göttingen, Germany 


\section{PROJECT II: VASCULAR RESPONSE TO SOCIAL}

\section{COGNITIVE PERFORMANCE MEASURED BY INFRARED \\ THERMOGRAPHY: A TRANSLATIONAL STUDY FROM \\ MOUSE TO MAN}

\subsection{Overview of project II}

Physiological measurements play an important role in psychological and neuroscientific research. Recently, advances in the field of infrared thermography (IRT) resulted in increased implementation attempts of this technology in studies investigating stress and arousal (Ermatinger et al., 2019; Gjendal et al., 2018; Herborn et al., 2018; Ioannou et al., 2014). Modern IRT is characterized by both high spatial and temporal resolution (Cardone \& Merla, 2017; Ioannou et al., 2014; Jarlier et al., 2011) and, due to its non-invasive and contact-free application method, can be employed where other 'conventional' techniques reach their limits (Ermatinger et al., 2019; Ioannou et al., 2014). However, various IRT studies suffer from diverse weaknesses, due to small and heterogeneous samples, insufficient control of the many environmental and personal confounding factors (Fernández-Cuevas et al., 2015), or suboptimal data extraction methods (Ioannou et al., 2014). Consequently, this leads to inconsistencies in findings (Ermatinger et al., 2019), limiting explanatory power.

This project seeks to enhance our knowledge of modern IRT technology and its potential in the assessment of stress, both in mice and men. We developed novel approaches to explore alterations in body surface temperature at defined regions of interest as readout of autonomous activity. Importantly, we took great effort to avoid the numerous pitfalls that typically occur in experimental IRT research (Cardone \& Merla, 2017; Fernández-Cuevas et al., 2015; Ioannou et al., 2014). This way, we intended to enhance the quality of collected data and analyses, ensuring robust study conclusions. 
The starting point of this work was the rather unexpected observation that mice exhibited abnormal social behavior during a modified Y-maze sociability test (Brimberg et al., 2016; Lai \& Johnston, 2002) four weeks after completing the SocioBox test of complex social recognition (Krueger-Burg et al., 2016). Intrigued by that finding we conducted more experiments, using mice, separated by sex, under two different conditions. While the experimental groups underwent the normal SocioBox paradigm, control groups stayed in the empty box. Four weeks afterward, all mice performed the Y-maze sociability test. As we were wondering whether groups differ regarding their autonomous activity, in addition to potentially distinct sociability behavior, we recorded body surface temperature via IRT. As hypothesized, experimental mice, in contrast to control animals, exhibited higher central body, compared to tail, temperatures over the course of the SocioBox experiment. Further, during the experimental test condition, thermal readouts were negatively associated with time spent interacting with the unfamiliar stimulus mouse, indicating that increased physiological arousal, as determined via IRT, interferes with recognition abilities.

In accordance with our initial findings, the Y-maze sociability test revealed both different behavioral as well as thermal readouts between experimental and control animals: While control mice showed a high motivation to interact with an unfamiliar conspecific, compared to a lifeless object, experimental group mice demonstrated a reduced social engagement. Moreover, experimental mice displayed higher body, compared to tail, temperatures. The evaluation of a possible relationship between thermal readouts during SocioBox and sociability during Y-maze showed that experimental mice with a higher thermal centralization during SocioBox spent less time interacting with a conspecific. Our findings suggest that the amount of physiological arousal experienced during the SocioBox is associated with a reduced motivation for social engagement, possibly inducing social avoidance behavior, which is maintained for at least four weeks.

In order to clarify whether the SocioBox is associated with overall behavioral changes, after the Y-maze sociability test all mice performed a paradigm of novelty-induced freezing. Importantly, comparisons in the degree of freezing behavior over time uncovered similar patterns between experimental and control mice. Based on these findings, we assume that the SocioBox is not only a promising instrument to assess complex social recognition memory in mice, it also seems to have a negative effect on social interaction behavior, 
indicating a potential value as a novel model of isolated social anxiety (Toth \& Neumann, 2013). IRT can be used to reliably record body temperature as an additional readout of physiological arousal, not revealed by conventional behavioral tests alone.

Subsequently, we evaluated the applicability of IRT in a human study, translating our findings from mice to man. Therefore, we created the Face Recognition Test (FRT), a social memory task using neutral male faces as stimuli (Kulke, Janßen, Demel, \& Schacht, 2017), that resembles, in its design, the SocioBox paradigm. We then employed IRT to explore temperature alterations between the FRT and the Wisconsin Card Sorting Test (WCST), a widely used test of executive functioning that employs abstract symbols (Heaton, Chelune, Talley, Kay, \& Curtiss, 2003), in a homogenous group of healthy men. In order to acquire solid data, novel approaches of automated extraction were employed. The analyses of thermal alterations over time in defined areas of the face revealed that the majority of participants showed a characteristic thermal pattern during the FRT but not in the WCST. Interestingly, salivary cortisol level changes were not different between the two tests and correlated rather mildly with thermal values. This suggests that IRT provides higher sensitivity than an established readout of physiological stress (Engert et al., 2014), at least within the context of the conducted social experiment.

\subsection{Original publication}

Seidel, J.*, Bockhop, F.*, Mitkovski, M.*, Martin, S., Ronnenberg, A., Krueger-Burg, D., Schneider, K., Röhse, H., Wüstefeld, L., Cosi, F., Bröking, K., Schacht, A., Ehrenreich, H. (2020). Vascular response to social cognitive performance measured by infrared thermography: A translational study from mouse to man. FASEB BioAdvances, 2, 18-32.

*Equally contributing authors

\section{Personal contribution}

I was, with the exception of the experimental data acquisition in mice, significantly involved in all aspects of both animal and human studies. Under the supervision of Prof. Hannelore Ehrenreich, and with contributions from my co-author colleagues, I was responsible for the design, conduction, and evaluation of the human experiments. In mice, and 
analogous in humans, I contributed to the process of quantitative data generation, which encompassed conversions of raw videos, identification and assignment of appropriate regions of interest as relevant readouts, and extraction of thermal and behavioral data for later analyses. Under the supervision of Prof. Hannelore Ehrenreich, I generated standardized preprocessing routines to obtain well-organized data in order to calculate new, intraindividually adjusted IRT readouts with the potential to methodologically enhance future thermal imaging research. I conducted and interpreted extensive statistical analyses, including behavioral, thermal, and cortisol level readouts. With contributions from my first-author colleagues, I wrote the Methods and Results sections as well as the Figure legends, created the Display items and was involved in the design of the Supplementary videos for the final manuscript. Together with my supervisor, I searched for relevant literature and wrote the Introduction and Discussion sections of the manuscript. 


\title{
Vascular response to social cognitive performance measured by infrared thermography: A translational study from mouse to man
}

\author{
Jan Seidel $^{1}$ ｜ Fabian Bockhop ${ }^{1}$ | Miso Mitkovski ${ }^{2}$ | Sabine Martin ${ }^{1}$ | \\ Anja Ronnenberg $^{1}$ | Dilja Krueger-Burg ${ }^{3}$ | Katharina Schneider ${ }^{2}$ | Heiko Röhse ${ }^{2}$ | \\ Liane Wüstefeld $^{1}$ | Filippo Cosi ${ }^{4}$ | Kai Bröking ${ }^{2}$ | Annekathrin Schacht ${ }^{5}$ \\ Hannelore Ehrenreich ${ }^{1}$
}

${ }^{1}$ Clinical Neuroscience, Max Planck Institute of Experimental Medicine, Göttingen, Germany

${ }^{2}$ Light Microscopy Facility, Max Planck Institute of Experimental Medicine, Göttingen, Germany

${ }^{3}$ Department of Molecular Neurobiology, Max Planck Institute of Experimental Medicine, Göttingen, Germany

${ }^{4}$ Biomedical Physics Group, Max Planck Institute for Dynamics and SelfOrganization, Göttingen, Germany

${ }^{5}$ Department of Affective Neuroscience and Psychophysiology, Georg-Elias-MüllerInstitute of Psychology, Georg-August University, Göttingen, Germany

\section{Correspondence}

Hannelore Ehrenreich, Clinical

Neuroscience, Max Planck Institute of Experimental Medicine, Hermann-ReinStr.3, 37075 Göttingen, Germany. Email: ehrenreich@em.mpg.de

\section{Funding information}

Max Planck Society; DFG Research Center for Nanoscale Microscopy and Molecular Physiology of the Brain (CNMPB)

\begin{abstract}
To assess complex social recognition in mice, we previously developed the SocioBox paradigm. Unexpectedly, 4 weeks after performing in the SocioBox, mice displayed robust social avoidance during Y-maze sociability testing. This unique "sociophobia" acquisition could be documented in independent cohorts. We therefore employed infrared thermography as a non-invasive method of stress-monitoring during SocioBox testing (presentation of five other mice) versus empty box. A higher Centralization Index (body/tail temperature) in the SocioBox correlated negatively with social recognition memory and, after 4 weeks, with social preference in the Y-maze. Assuming that social stimuli might be associated with characteristic thermo-responses, we exposed healthy men $(\mathrm{N}=103)$ with a comparably high intelligence level to a standardized test session including two cognitive tests with or without social component (face versus pattern recognition). In some analogy to the Centralization Index (withinsubject measure) used in mice, the Reference Index (ratio nose/malar cheek temperature) was introduced to determine the autonomic facial response/flushing during social recognition testing. Whereas cognitive performance and salivary cortisol were comparable across human subjects and tests, the Face Recognition Test was associated with a characteristic Reference Index profile. Infrared thermography may have potential for discriminating disturbed social behaviors.
\end{abstract}

\section{KEYWORDS}

flushing, IRT, social stimulus, stress, temperature, vasoactivity

\footnotetext{
Abbreviations: BSI, Brief Symptom Inventory; FRT, Face Recognition Test; HPA, Hypothalamic-Pituitary-Adrenal Axis; IRT, Infrared Thermography; LOESS, Locally Estimated Scatterplot Smoothing; LPS, Leistungsprüfsystem; $m K$, Millikelvin; NEO-FFI, NEO-Five-Factor Inventory; NETD, Noise Equivalent Thermal Difference; ROI, Regions Of Interest; SIAS, Social Interaction Anxiety Scale; SPS, Social Phobia Scale; STAI, State-Trait Anxiety Inventory; WCST, Wisconsin Card Sorting Test.

Jan Seidel, Fabian Bockhop and Miso Mitkovski are equally contributing first authors.

This is an open access article under the terms of the Creative Commons Attribution-NonCommercial License, which permits use, distribution and reproduction in any medium, provided the original work is properly cited and is not used for commercial purposes. (c) 2019 The Authors.
} 


\section{1 | INTRODUCTION}

An association of emotions during unaccustomed social interactions with facial flushing in humans has long been recognized. ${ }^{1-3}$ The autonomic nervous system response during such social interactions - highly conserved across mammals and perceived like "stress"-leads to altered vasoactivity in peripheral and core body regions. The resulting blood flow changes via vasoconstriction and vasodilation, respectively, affect local body temperature. ${ }^{4-10}$ Exposure to an embarrassment task, for instance, led to an increase in facial blood flow in both male and female participants, measured via Laser Doppler Flowmetry. ${ }^{11}$ Using this technique, temperature changes upon sympathetic vasoconstriction, occurring with a delay of 5-15 s, ${ }^{12-14}$ can be reliably detected. Negative as well as positive social stimuli provoke alterations in surface temperature of various facial areas, with the nose consistently reported as highly reactive to affective and social cues. ${ }^{12,15-18}$

These observations advocate infrared thermography (IRT) as a highly attractive method of contact-free and non-invasive measurement of naturally emitted electromagnetic radiation with a wavelength between $0.75-1000 \mu \mathrm{m}$, commonly interpreted as "heat". ${ }^{19}$ Modern IRT recording systems are characterized by high spatial and temporal resolutions and require almost no restrictions in movement of test subjects, allowing a more natural/ecological testing environment. ${ }^{9,15,20}$ Because of its high accuracy, relative ease of use, and minimal inconvenience for the subjects, IRT has already been implemented in different fields of medical research and practice. ${ }^{19,21}$

While the validity of IRT for assessing surface temperature is generally accepted and has led to several pivotal publications, ${ }^{4,6,7,16,22-28}$ its broader applicability in the future will depend on controlling environmental, subject-related, and technological factors ${ }^{29}$ as well as improved reliability and reproducibility. So far, no overall accepted, dependable state-ofthe-art procedure for IRT testing in social behavior diagnostics has been introduced. Numerous different experimental designs, test stimuli, facial/body target regions, and data extraction/ analysis procedures have been reported. ${ }^{9,21}$ Often, single or short series of IRT images (before versus after experimental condition) are described, based on rather small and heterogeneous samples, whereas data on thermal dynamics over longer time intervals are scarce. Interpretation of thermal alterations is frequently limited to single directional statements, for instance increase or decrease or unaltered temperature.

In the present translational study, we employ and adapt IRT for more reliable, internally controlled measurement of a social stimulus-related autonomic vaso-response. We start with an unexpected discovery in mice, namely induction of "sociophobia" upon inescapable interaction in a social recognition test, where the Centralization Index (ratio body/ tail temperature) serves as continuous "whole body stress readout". We then extend these findings to human subjects, exposed to social versus non-social cognitive tasks in a highly standardized fashion. Here, the Reference Index (ratio nose/malar cheek temperature) is introduced to determine the autonomic facial response/flushing during social recognition testing. We report a novel non-invasive "sociophobia" model in mice, characterized by a pronounced thermo-reaction during induction and on retrieval, and a typical facial thermo-response in men under cognitive challenge containing a social component.

\section{2 | MATERIALS AND METHODS}

\section{1 $\quad$ Mouse studies}

\subsection{1 | Mice}

All experiments were approved by and conducted in accordance with the regulations of the local Animal Care and Use Committee (Niedersächsisches Landesamt für Verbraucherschutz und Lebensmittelsicherheit, LAVES). C57BL/6JRj mice were used as experimental mice, $\mathrm{C} 3 \mathrm{H} /$ $\mathrm{HeNCrl}$ as stimulus mice (Charles River). Animals were group-housed in standard cages $(36.5 \times 20.7 \times 14 \mathrm{~cm}, 4-5$ mice per cage of the same gender and strain), in rooms separated by gender and strain (to avoid olfactory contact), and kept on a $12 \mathrm{~h}$ light-dark cycle (lights off at $7 \mathrm{PM}$ ) at $20-22^{\circ} \mathrm{C}$. Food and water were provided ad libitum.

\subsection{2 | SocioBox test for complex social recognition memory and recording}

A detailed description of the SocioBox as a multiple social recognition task is provided elsewhere ${ }^{30}$ (see also Figure 1). Experiments were conducted during light phase of the day (10-15 lux, $23.5^{\circ} \mathrm{C}$ room temperature), with male or female C57BL/6JRj experimental mice $(\mathrm{N}=45$ in total $)$ and gendermatched $\mathrm{C} 3 \mathrm{H} / \mathrm{HeNCrl}$ as stimulus mice (Figure 1A). Male mice were 13-15, female 20-22 weeks old. Prior to test session, experimental and stimulus mice had been habituated separately (in absence of any other mice) to the SocioBox for 3 consecutive days. The following test sessions consisted of three phases, namely exposure 1 , exposure 2 , and recognition test. $^{30}$ At beginning of test session, the experimental mouse was placed into the central arena inside a white Plexiglas circular partition, spatially and visually separated from stimulus mice. After 5 minutes of recovery ("Initiation stage"), the circular partition was lifted, and the mouse allowed to freely explore the arena, including the stimulus mice in their inserts, for 5 additional minutes ("Interaction stage"). At the end of exposure 1 , the mouse was removed and placed back in its transport cage. The arena was cleaned and the mouse then 
(A) SocioBox with $\mathrm{C} 3 \mathrm{H}$ stimulus (B) Y-maze sociability design mice and test mouse in center
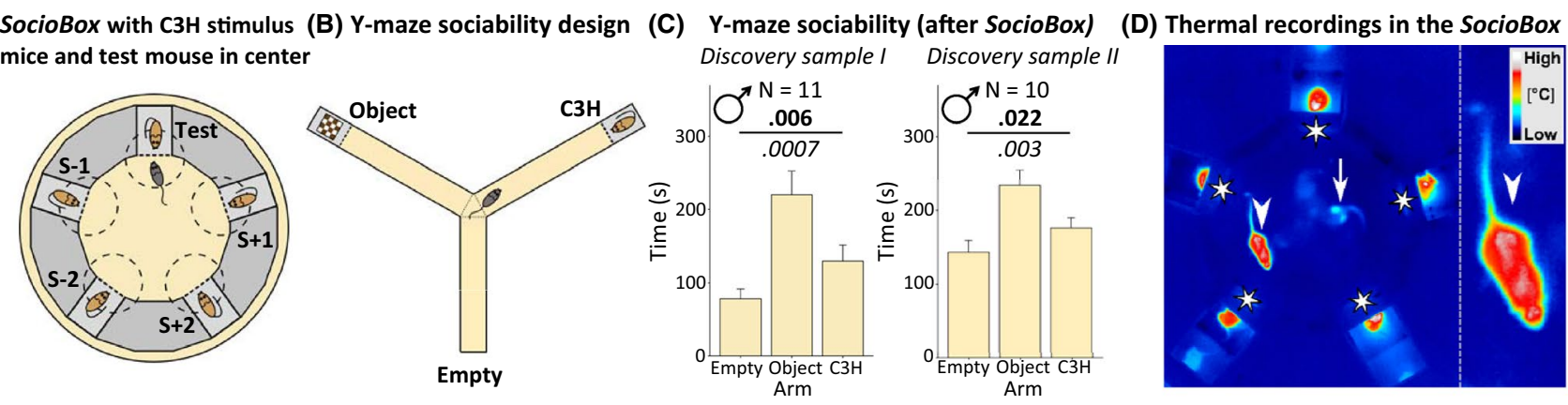

(E) SocioBox: Recognition
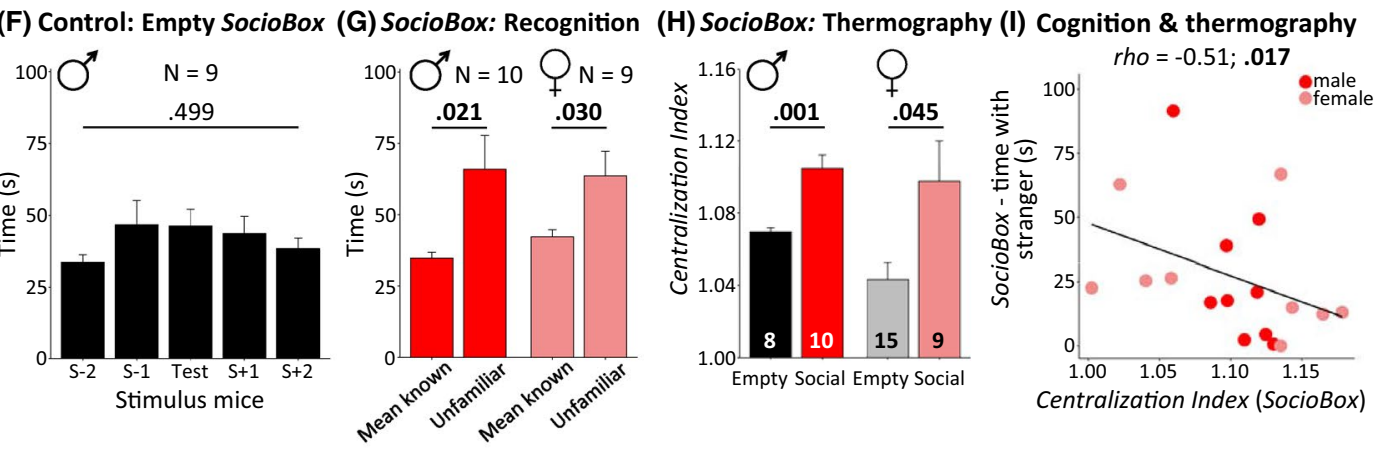

(J) Thermal recordings in Y-maze

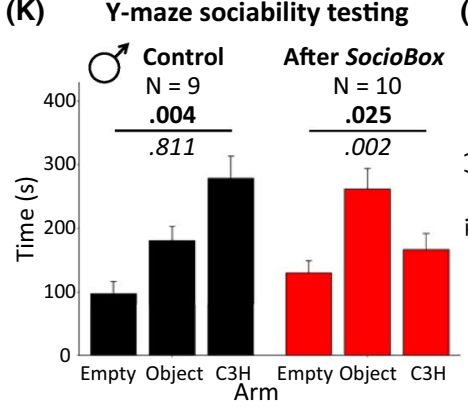

(L) Y-maze sociability testing
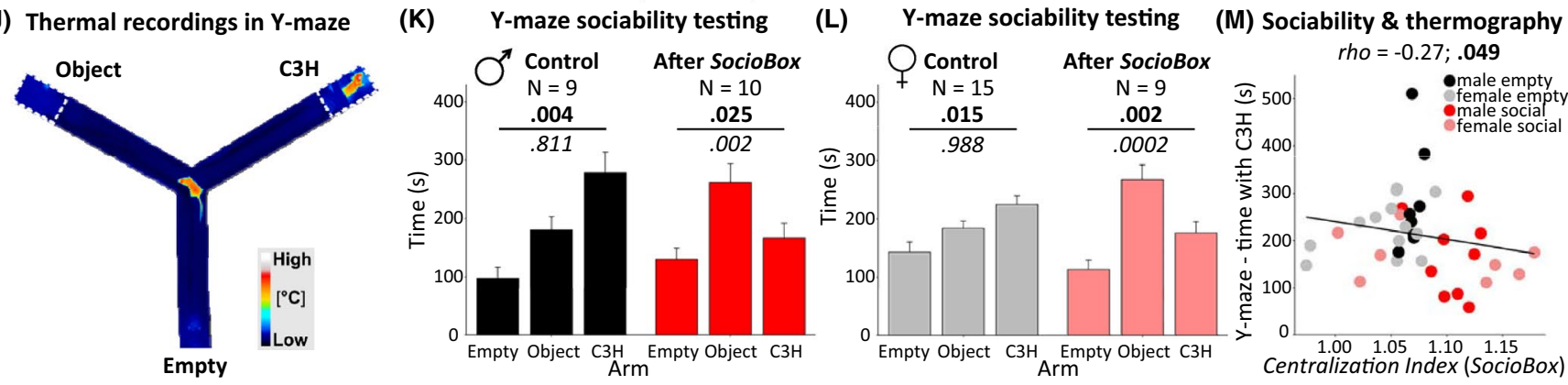

F I G URE 1 Mouse IRT study: SocioBox recognition testing induces lasting social avoidance in mice. A, SocioBox arena ${ }^{30}$ with experimental mouse in center (gray), unfamiliar "stranger" ("Test") and familiar stimulus mice (all brown). Time spent in zones close to each stimulus mouse (circles) is recorded to determine interaction/recognition. B, Y-maze sociability test: ${ }^{31}$ Test mouse starts in center of Y-maze with one arm empty, one containing an object and the third another mouse $(\mathrm{C} 3 \mathrm{H})$. Time spent in each arm is measured. A normal naïve mouse spends most of the time with the other mouse (stair pattern of controls in panel K and L). C, Unexpectedly, 4 weeks after SocioBox testing, experimental mice display social avoidance behavior in 2 independent samples: Discovery I and II; repeated-measure ANOVA; quadratic-trend analysis (below; italics). D, IRT image of SocioBox test with experimental mouse (arrowhead) and stimulus mice (asterisks); white arrow: experimental mouse left trace of urine (evaporation cooling). Magnification on the right illustrates temperature differences in body parts. Compare video S1. E, Zone preference of male mice during SocioBox recognition testing. Mice with normal recognition memory spend most time with the "stranger" (unfamiliar stimulus mouse). F, Control mice tested in empty SocioBox do not show appreciable zone preference; repeated-measure ANOVA. G, Average interaction time with all familiar mice versus time with unfamiliar mouse (stranger); paired Student's $t$ tests (one-sided). H, Both genders exhibit in SocioBox an increase in Centralization Index (body/tail temperature), compared to controls in empty box; unpaired Student's $t$ tests (two-sided). Note that due to difficulties in tracking tail ROI ( $\geq 25 \%$ missing values), 3 animals ( 1 male, 2 female controls) had to be excluded from thermal analyses. I, Centralization Index is negatively correlated with social recognition performance; Spearman's rho (one-sided). J, Representative IRT image during Y-maze sociability. K-L, Both genders show robust social avoidance 4 weeks after SocioBox compared to the expected stair pattern of control animals; repeated-measure ANOVA; quadratic-trend analysis (below; italics). Due to atypical hypoactive behavior during testing, two female control animals were excluded prior to analyses. M, Social aversion priming: Negative correlation between Centralization Index in SocioBox and time spent with $\mathrm{C} 3 \mathrm{H}$ conspecific in Y-maze 4 weeks later; Spearman's rho (one-sided)

again placed in the SocioBox center. Exposure 2 followed the same procedure. At the end of exposure 2, one of the five stimulus mice was randomly exchanged for a new, unfamiliar conspecific. Next, the mouse was reintroduced and the recognition test conducted accordingly. During the course of the experiment, a black body-calibrated A655sc IRT camera with a $13.1 \mathrm{~mm}$ focal length lens was used. The system has a noise equivalent thermal difference (NETD) $<30 \mathrm{mK}$ and resolution of $640 \times 480$ pixels (FLIR ResearchIR Max software v4.40.2.1, TOPA, Hohenpeissenberg, Germany) and was mounted $110 \mathrm{~cm}$ above the arena, recording at a framerate of $25 \mathrm{~Hz}$. Care was taken that no direct or indirect heat 
emissions from external sources affected recordings. The IRT camera was connected to a computer located in a separate room. Readouts were temperature changes of the mouse, duration of interaction with stimulus mice/recognition of the stranger mouse, and distance traveled (video S1). To ensure that sociability changes are not triggered by the SocioBox arena itself, the same procedure was conducted with control mice exposed to an empty SocioBox (without stimulus mice).

\subsection{3 | Y-maze sociability testing and recording}

Y-maze testing was performed as described with slight modifications. ${ }^{31}$ Mice were tested 4 weeks after SocioBox/empty box performance on 2 consecutive days at light intensities of 60-70 lux. Day 1 included three habituation trials with an inter-trial-interval of $60 \mathrm{~min}$. The mouse was placed in one of the arms and allowed to explore the empty maze for $10 \mathrm{~min}$ per trial. The starting point was rotated through all three arms (dimensions of each arm $46.1 \times 8.3 \times 13.7 \mathrm{~cm}$ ). On day 2 , an object ( $6 \mathrm{~cm}$ chess piece) and a $\mathrm{C} 3 \mathrm{H}$-stranger mouse, same gender and age, were each presented in an insert, preventing direct access, and positioned at the end of two randomly chosen Y-maze arms while the third arm remained empty (Figure 1B). The mouse was then placed in the empty arm, facing the center, and allowed to explore freely for 10 minutes. All mice underwent the same test procedure. The IRT camera was positioned $130 \mathrm{~cm}$ above the maze, readouts were changes in temperature, distance traveled and, to estimate social preference, time spent in each arm (Figure 1J).

\subsection{4 | Data extraction and preprocessing}

Mouse location and stress readouts during SocioBox recognition and Y-maze sociability tests were assessed through an image analysis workflow implementing the software packages Ilastik v.1.3.3b $2^{32}$ and FIJI, ${ }^{33}$ as well as the TrackMate ${ }^{34}$ FIJI plugin. Thermal readouts of both, body and tail, were extracted by first using the pixel classification workflow of Ilastik. Pixel groups delineating "background", "body", and "tail" were annotated to train a Random Forest classifier $^{32}$ that was used to produce probabilities for the respective classes for each image sequence of the recorded mice. Resulting body and tail probabilities were binarized with FIJI to generate masks, which were then applied to the corresponding, original IRT image sequence as regions of interest (ROI), from which the relative mean body and tail temperatures were obtained.

For the SocioBox, five zones were defined in close proximity to each stimulus mouse/empty inset (Figure 1A). Number of frames the respective mouse spent in each zone was summed up to obtain total interaction time with stimulus mice. Interaction with stranger (unfamiliar mouse) served as readout of social recognition. ${ }^{30}$ To exclude zone preferences not attributable to experimental setup (eg, room features) during empty SocioBox condition, control mouse zone orientations were randomly matched to experimental mouse zones. A similar procedure was employed during Y-maze sociability, counting number of frames in each arm (empty, object, $\mathrm{C} 3 \mathrm{H}$ ).

After down-sampling $(1 \mathrm{~Hz})$ to increase computational speed during following preprocessing steps, frames with missing information (eg, hidden tail) were replaced by the mean of the remaining data points for each mouse. Mice with $\geq 25 \%$ missing values were excluded from respective thermographic analysis. To reduce random noise effects we smoothed data sequences of both body and tail separately, using locally estimated scatterplot smoothing (LOESS). By dividing the relative mean temperature of the body ROI by its corresponding tail ROI relative mean temperature at each time point, we created an intraindividually adjusted measure of endogenous arousal: the Centralization Index. To evaluate whether potential thermal differences were independent of higher physical activity, we additionally calculated the distance mice traveled in $500 \mathrm{~ms}$ intervals for SocioBox and Y-maze.

\section{2 $\quad$ Statistical analyses}

Both male and female experimental (SocioBox) versus control (empty box) mice were analyzed. To reduce the impact of extreme values in statistical analyses while avoiding exclusion, data for each group (empty SocioBox, SocioBox with stimuli) were winsorized: extreme values $<5$ th and $>95$ th percentiles were set to 5 th and 95 th percentiles, respectively. ${ }^{35}$ Total time spent in SocioBox zones was analyzed using repeated measure analyses of variance (ANOVA). ${ }^{30}$ Additionally, for experimental groups, average time spent in zones with familiar mice was compared with time spent with stranger (unfamiliar zone). Due to expected outcome (more time spent with unfamiliar mouse), ${ }^{30}$ one-sided paired Student's $t$ tests were calculated. Differences in mean Centralization Index and total distance traveled were compared between conditions via two-sided unpaired Student's $t$ tests. Exploring the relationship between Centralization Index as readout of physiological reactivity (stress) and recognition performance, Spearman's rho was calculated for all experimental mice. Because of initial orientation and adaption to the situation with potentially interfering effects on recognition performance, we used only the second half of SocioBox test (minutes 4-5). Hypothesizing that a higher Centralization Index is associated with worse performance, analysis was one-sided. For Y-maze sociability, differences in time spent in each arm was tested via repeatedmeasure ANOVA with following linear and quadratic trend analyses using polynomial contrasts. Both differences in 
mean Centralization Index and total sum of distances traveled were analyzed with two-sided unpaired Student's $t$ tests. Hypothesizing that the Centralization Index in Y-maze correlates negatively with sociability, one-sided Spearman's rho was calculated, including all test mice. Additionally, to investigate the relationship between severity of experience during SocioBox (assumed priming of social aversion) and sociability in Y-maze, we calculated Spearman's rho for Centralization Index in SocioBox and time spent in $\mathrm{C} 3 \mathrm{H}$-arm during $\mathrm{Y}$-maze, again with test mice from all conditions. Expecting a negative correlation, a one-sided test was applied. All statistical analyses were performed using R v3.5.2 $2^{36}$ with RStudio v1.1.463 (RStudio Inc, Boston, United States) and significance level of alpha $=0.05$. Welch-corrected Student's $t$ tests were used, and, in cases of violations of sphericity, Greenhouse-Geisser corrections were applied to repeated-measure ANOVA.

\section{3 $\quad$ Human studies}

\subsubsection{Participants}

The study was conducted in accordance with the Declaration of Helsinki and approved by the ethics committee of the Georg-Elias-Müller-Institute of Psychology, University of Göttingen. Online screening was set up to attract and assess eligibility of potential participants. Besides providing demographic information and answering questions regarding their ability to identify and memorize faces, interested individuals completed the German versions of Brief Symptom Inventory $(B S I)^{37}$ and complementary social phobia instruments Social Phobia Scale (SPS) and Social Interaction Anxiety Scale $(\mathrm{SIAS})^{38}$. As additional readout of personality structure, NEO-Five-Factor Inventory (NEO-FFI) ${ }^{39}$ was filled out. Those with questionnaire scores within normal limits were invited to the experimental session, aiming to include only mentally healthy individuals without indication of (sub)clinical symptoms. Based upon results of this online screening, a total number of $\mathrm{N}=111$ subjects were invited. However, due to psychiatric conditions, illicit substance consumption shortly before study onset, or technical difficulties during recording, $\mathrm{N}=8$ had to be excluded, leaving a final sample of $\mathrm{N}=103$ participants (see Figure 2A for inclusion process). All subjects were heterosexual, native German men between 18 and 34 years of age with normal or contact lens-corrected vision, no facial piercings or beard, and without history of neuropsychiatric or somatic diseases.

\subsection{2 | Experimental procedure}

To reduce impact of external factors during IRT recordings $^{9,15,29}$ participants were asked to avoid alcohol consumption $(24 \mathrm{~h})$, physical activity $(12 \mathrm{~h})$, and intake of food or activating substances (eg, caffeine, nicotine; $2 \mathrm{~h}$ ) before test session (Figure 2 and Figure 3). Additionally, they were instructed not to shave or apply facial lotion at testing day. Study participation was compensated with $35 €$ or course credit. Completing the online screening offered the chance to win 1 of 3 gift cards (10€). All test subjects gave written informed consent and could withdraw participation at any time. Main experiments took place in a $5 \times 3 \mathrm{~m}^{2}$-sized testing room without direct sunlight or ventilation and with normal ambient temperature $\left(\mathrm{M}=22.94^{\circ} \mathrm{C}, \mathrm{SD}=1.14\right)$ and humidity ( $\mathrm{M}=59.62 \%, \mathrm{SD}=7.34)$. Trained experimenters (JS, FB) ensured standardized test conditions during sessions, ${ }^{29}$ which consisted of an initial assessment, habituation, two IRT-recorded computer tests of cognitive abilities, and a closing assessment (Figure 2B). Individual sessions consistently started at either 09:00 AM or 11:00 AM, total test duration did not exceed 120 minutes. During initial assessment, the participant was welcomed and informed about study procedure, followed by an examination of state-trait anxiety (German version of State-Trait Anxiety Inventory, STAI) ${ }^{40}$ and, thereafter, general face perception abilities via a short prosopagnosia test. IRT-recorded cognitive testing was performed in a $3 \times 2 \mathrm{~m}^{2}$-sized chamber within the experimental room (Figure 3A). One chamber side was not completely closed to allow fresh air supply and communication between subject and experimenters. The participant was seated in a specialized comfortable chair which adapted to his body size and shape, effectively avoiding pressure points $\left(\mathrm{M}^{\mathrm{C}} \mathrm{Lean}\right.$ REHAtechnik, Duderstadt, Germany). The implemented headrest enabled relaxation of head and neck muscles, while gently minimizing head movements (Figure 3A). The entire setup was highly adaptable to the differing subject shapes and sizes preventing irritation of the vascular system, while ensuring an unobstructed view of the relevant facial features for the IRT camera. Prior to testing, subjects stayed in a relaxed position for 15 minutes to acclimatize to setup (habituation phase). ${ }^{15,29}$ Each participant performed computerized tests of both executive functioning and social cognition in counterbalanced order, separated by a 2 min break. The IRT camera was mounted above the monitor and recorded whole-face images of the participant at $25 \mathrm{~Hz}$ from approximately $32 \mathrm{~cm}$ distance while the subject performed the tasks.

\section{Executive functioning}

Executive functioning as a process of general cognitive abilities was measured via Wisconsin Card Sorting Test, Computer Version 4 (WCST). ${ }^{41}$ Subjects are required to virtually classify cards regarding different features (symbol, number, color) via button press to 1 of 4 target decks. No additional instructions are provided. Instead, subjects have to infer sorting strategies from a feedback ("correct"/"incorrect") following each sorting decision. After a series of correct answers 


\section{(A) Recruitment flow}

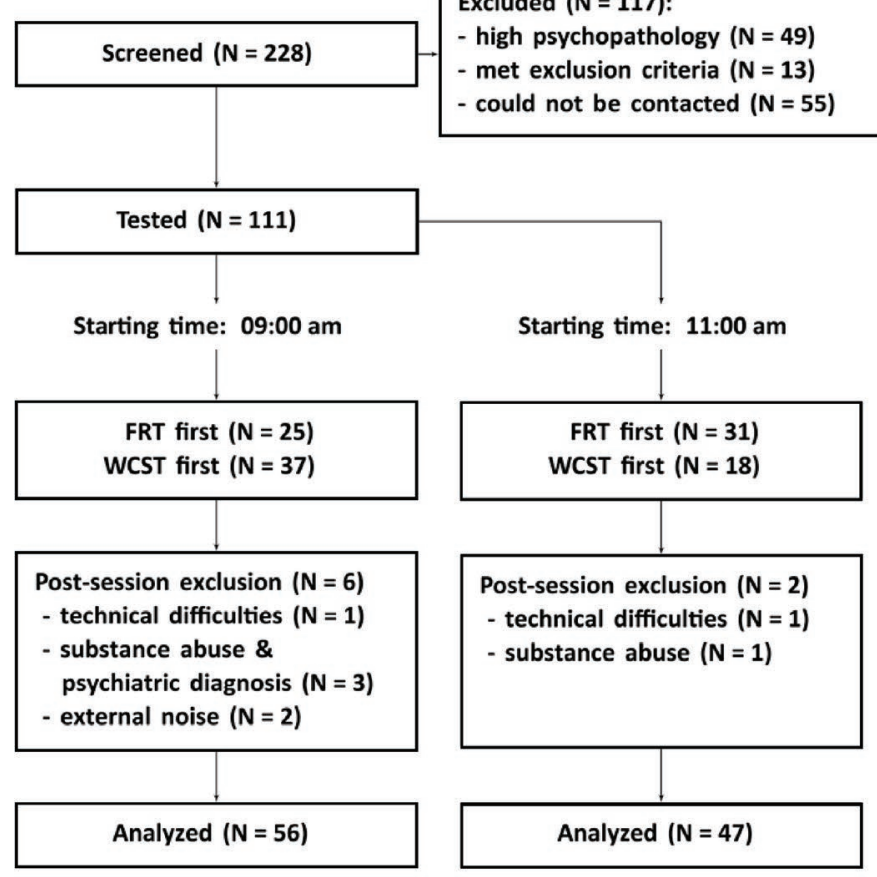

(C) Design: Face Recognition Test (FRT)
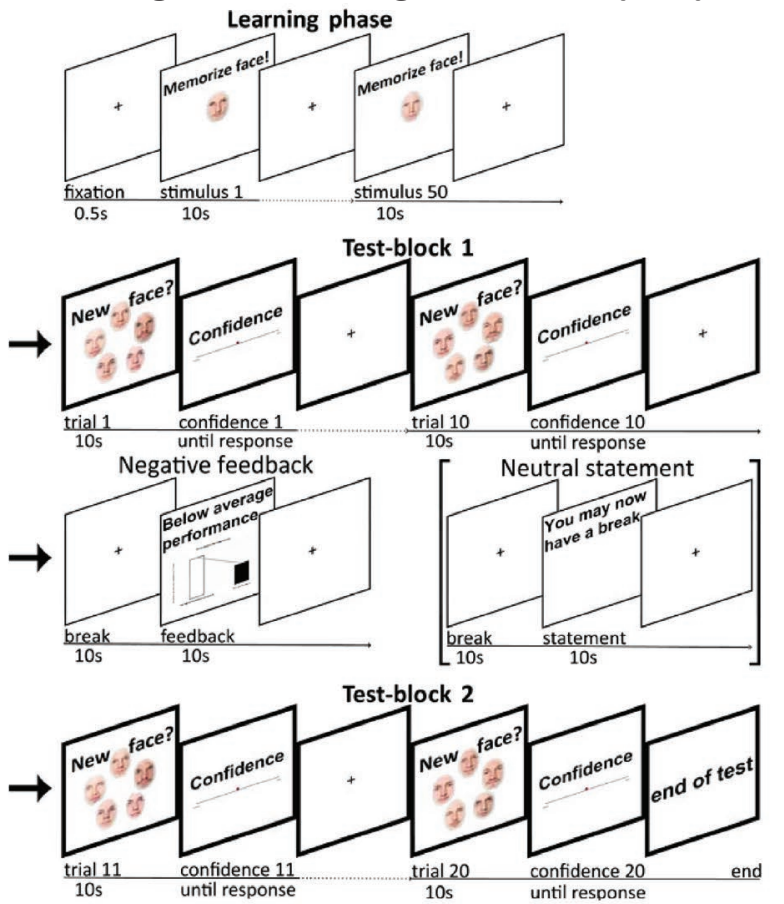

(B) Study design

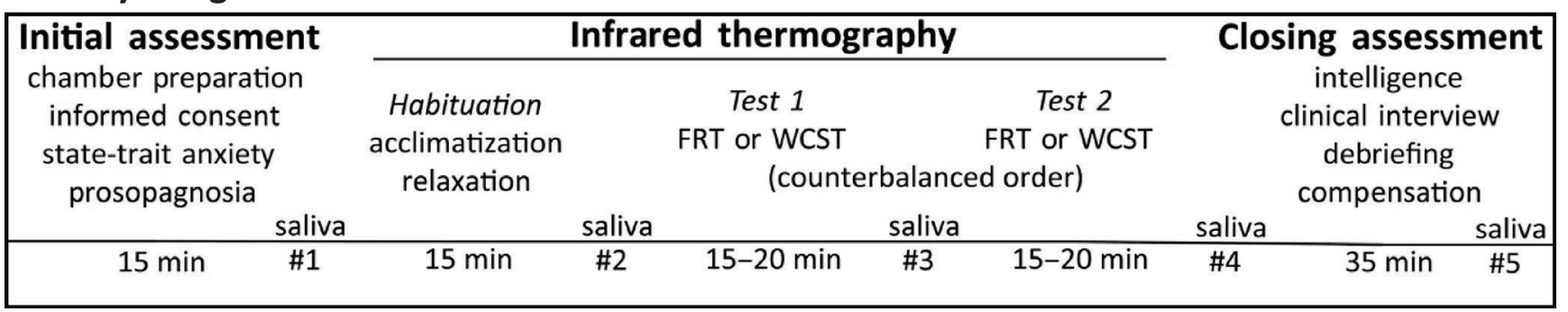

F I G U RE 2 IRT of social recognition versus executive function testing in men: Study logistics. A, Recruitment process: To ensure sample homogeneity, pre-experimental online screenings were implemented. A total of 111 individuals completed experimental session, starting at either 09:00 AM or 11:00 AM. After post-session exclusion, 103 subjects remained as final sample. B, Study design: During initial assessment, participants were welcomed and provided with study information, followed by state-trait anxiety and prosopagnosia examinations. Then, IRT-recorded test phase was conducted, starting with habituation and 2 computerized cognition tests (in counterbalanced order: FRT, WCST). At closing assessment, intelligence was measured, and an interview concerning mental and physical health as well as debriefing (explanation of feedback) and, finally, compensation for participation took place. Over the course of experimental session saliva samples for cortisol analysis were collected at 5 different time points in 15-35 min intervals. C, Sketch of the novel, brief Face Recognition Test (FRT): Participants are first asked to memorize 50 male stimulus faces (learning phase). Subsequently, test trials containing 5 stimulus faces each are presented; subjects decide whether all are familiar or not, respectively (test-block 1). After 10 trials either a fabricated, negative feedback or an alternative, neutral statement is presented, before concluding with 10 more trials (test-block 2), analogous to test-block 1

of certain length, sorting criteria shift, prompting subjects to adapt.

\section{Social cognition}

Social cognition in humans was assessed based on face memory tasks. ${ }^{42-44}$ Analogous to the SocioBox recognition test in mice, we created the Face Recognition Test (FRT), a computerized measure of social cognitive abilities (face perception, face memory based on internal features, perception of social evaluation). The FRT consists of a learning phase, followed by two test blocks, each separated by a negative feedback or, alternatively, neutral statement (Figure 2C). The test is presented via PsychoPy v1.85.4 ${ }^{45}$ for Python v2.7. ${ }^{46}$ During the learning phase, 50 male faces from the Göttingen Faces Database ${ }^{47}$ with neutral valence, standardized visual features, luminance, and resolution are sequentially presented in random order. Test subjects are instructed to stay calm, focus and memorize the stimulus on screen. Each face is shown once for $10 \mathrm{~s}$ at the center of the screen, followed by fixation cross $(500 \mathrm{~ms})$. Learning phase lasts $560 \mathrm{~s}$ in total. For test-block 1 , of the previous 50 stimulus faces, two are randomly replaced with unfamiliar ones. This new set of images is then given in 10 trials. Each trial includes 5 stimuli, randomly presented in a circular order (Figure 2C). Participants indicate via keyboard button press within $20 \mathrm{~s}$ if 


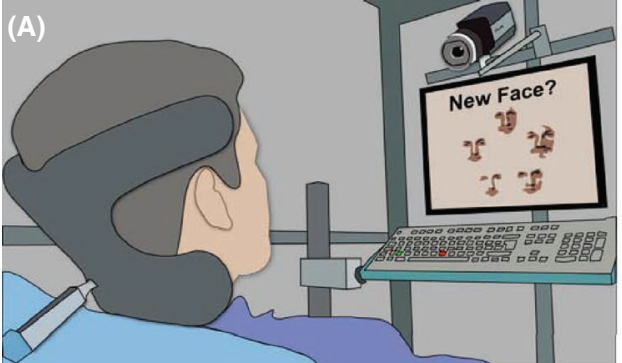

(B)

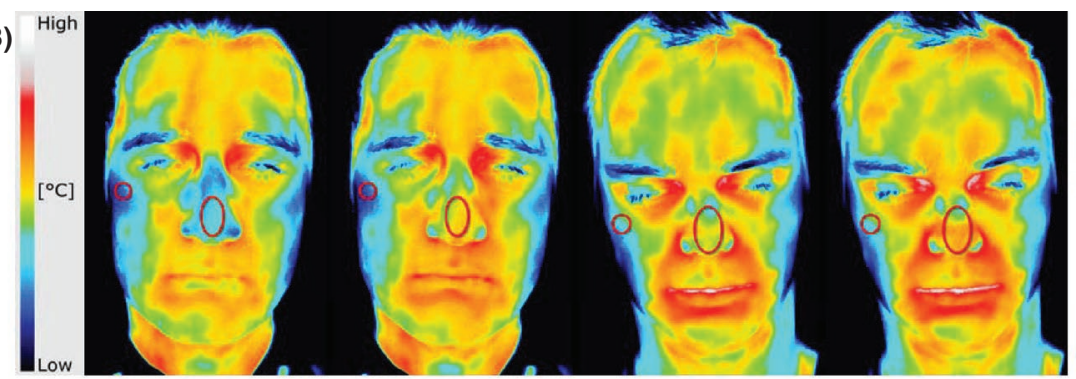

(C) Thermal patterns, FRT (RI) All individuals shown

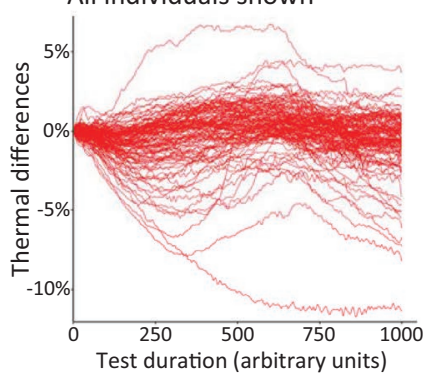

(F) Different thermal pattern of tests (Reference Index, RI)

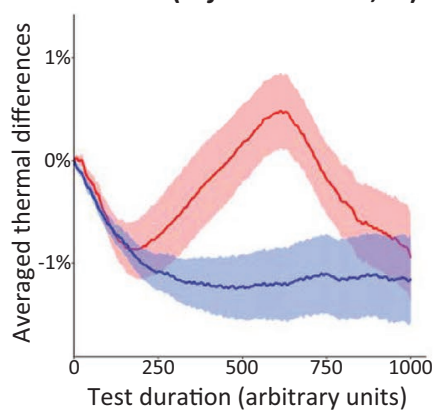

(D) Thermal patterns, WCST (RI) (E)

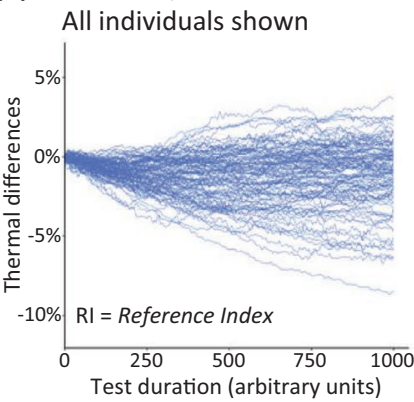

(G) Thermal dynamics -

fluctuations over time

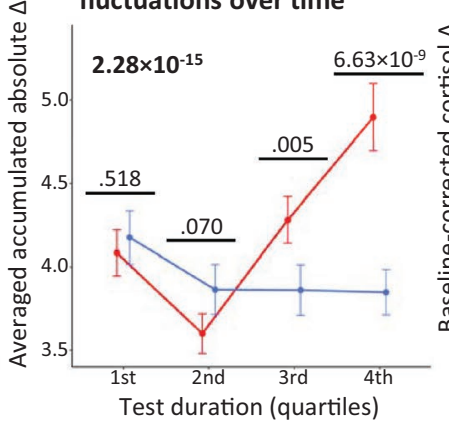

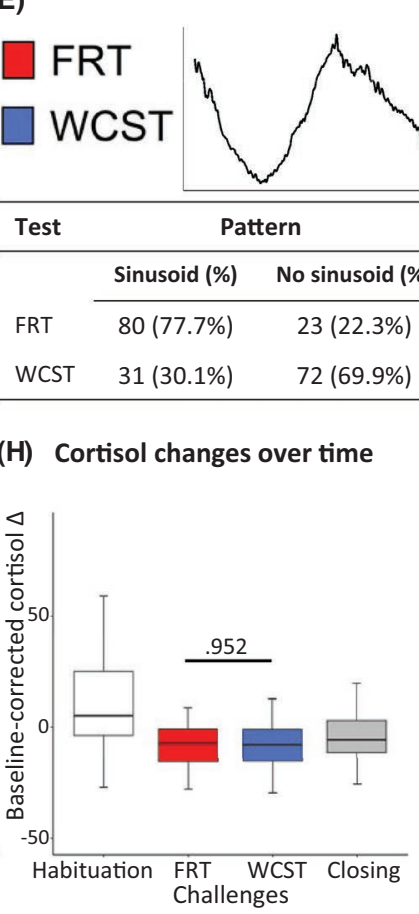

$1.96 \times 10^{-11}$

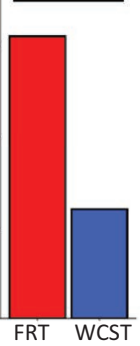

(I) Cortisol $\Delta$ values \& thermography

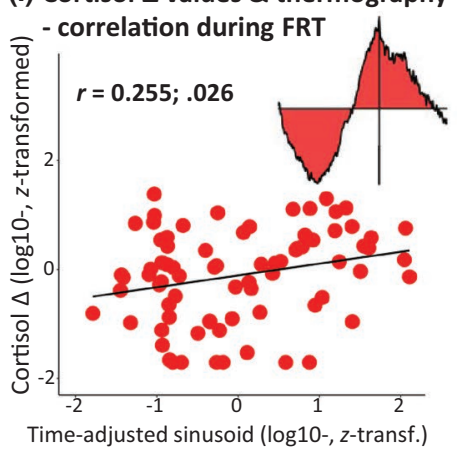

F I G URE 3 Human IRT study during social recognition versus executive function testing: Social cognition stimulus induces a distinct thermo-pattern. A, Illustration of test setup: Participant sits as still as possible in a comfortable orthopedic armchair, with headrest to minimize head movements, and performs FRT (Face Recognition Test) while IRT camera (above screen) records facial regions of interest (ROI). B, IRT images of two sample test subjects with nose and right malar cheek ROI (circled in red) used to calculate Reference Index (RI). Images taken from early and late FRT session phase, respectively. Compare video S2. C-D, Overlays of all participants' normalized Reference Index differences from baseline in both tests indicate a sinusoid-shaped thermo-pattern over the course of FRT but not WCST (Wisconsin Card Sorting Test). E, Consensus ratings by three examiners (blinded to any test/subject information) revealed that the majority of test subjects exhibited a characteristic Reference Index sinusoid pattern during FRT (left example curve) but not WCST (right example curve); $\chi^{2}$-test. F, Comparison of Reference Index course between tests over time using $\mathrm{M} \pm 95 \%$ confidence intervals. Groups' thermo-patterns differ significantly where confidence intervals do not overlap. G, Over each test quartile, accumulated absolute changes in Reference Index showed differences during second test halves, with higher temperature dynamics in FRT compared to WCST; repeated-measure ANOVA, Bonferroni-adjusted multiple-comparison tests (two-sided). H, Contrary to Reference Index, salivary cortisol reactivity was similar between tests, suggesting IRT as a more sensitive tool for measurement of physiological responses in social tasks. Cortisol levels were log10-transformed and normalized to the first sample (baseline); then cortisol delta values were calculated between sample time points; paired Student's $t$ test (two-sided). I, Pearson correlation coefficient revealed mild-to-moderate positive relationship between $\log 10$ - and $z$-transformed time-adjusted integrals of Reference Index and $z$-transformed salivary cortisol delta values during FRT. Only participants with characteristic sinusoid thermal curve and $z$-score \pm 2.58 included $(\mathrm{N}=78)$

all faces are familiar or not. Each stimulus face is shown only once and trials never contain more than one unfamiliar face. Additionally, after each trial, participants rate confidence in their response on a 5-point Likert scale. Then, after a break of $10 \mathrm{~s}$, either an unprompted, fabricated negative feedback or a neutral statement is included, each lasting for $10 \mathrm{~s}$. Negative feedback is shown graphically and as text ("your performance is below average"), the non-threatening alternative is displayed as text ("you may now take a break - the second part of the test will start automatically"). Immediately thereafter, test-block 2 is initiated with the two unfamiliar faces of testblock 1 again exchanged for two new faces and participants perform the same task with confidence judgments, analogous to test-block 1. Closing assessments covered a structured clinical interview on mental and somatic conditions, a nonverbal intelligence assessment (performance test system subtest-3; 
"Leistungsprüfsystem Untertest-3"), ${ }^{48}$ monetary compensation and, lastly, a debriefing on the aims of the study. As an additional, biological readout, saliva was collected to measure cortisol levels at five different time points in intervals of around 15-35 min (Figure 2B).

\subsubsection{Data extraction \& preprocessing}

Tracking information of facial ROI in human subjects was obtained with the DeepLabCut software package ${ }^{49}$ and implemented into a FIJI-based image analysis workflow, allowing for corrections of small head movements not prevented by the headrest. Labels delineating either nose or right cheek (malar region) coordinates (Figure 3B; video S2) in up to 600 images were used to train the DeepLabCut network. Facial regions were selected regarding reactivity to social stimuli, with nose reacting strongly while malar cheek does not. After $10^{6}$ iterations, the resulting network had converged sufficiently to be evaluated for accuracy and then applied to human IRT recordings in FIJI in order to track and extract relative mean temperatures of the two facial ROI at their respective position and time. The resulting series of temperature values ( 25 per second) were subsequently down-sampled $(1 \mathrm{~Hz})$ for following processing steps. In order to replace missing values we calculated the sequence (ascending/descending) between the last valid data points before and after the missing. Then both ROI frame sequences were smoothed separately using LOESS fitting.

By dividing mean nose ROI temperature of each frame with its corresponding malar cheek ROI, we calculated the Reference Index, analogous to the Centralization Index in mice. Due to initial temperature differences in respective ROI, Reference Index was normalized as percentage change from the very first frame (baseline), and, due to varying individual test length of participants, duration of both tests was normalized to 1000 arbitrary units, both for FRT (Figure 3C) and WCST (Figure 3D). To investigate differences in thermal curve characteristics, two independent evaluators rated in a blinded manner over both tests whether the normalized Reference Index curve was initially decreasing and then increasing (sinusoid curve), or was differently shaped (Cohens' kappa $=0.63$ ). In case of dissent, a third evaluator made a final decision on the rating.

\subsection{4 | Saliva cortisol determination}

Saliva was collected at five different time points in intervals of 15-35 minutes (Figure 2B) and stored at $-80^{\circ} \mathrm{C}$ until further use. ELISA was used to detect cortisol levels in saliva samples, according to manufacturer's instruction (Demeditec, Kiel, Germany). To account for circadian cortisol profile differences (ie, cortisol awakening response ${ }^{50-52}$ ) due to experimental starting points (09.00 AM versus $11.00 \mathrm{AM})$, we calculated normalized delta values $(\Delta)$ between sample collection time points: First, all samples were log10-transformed and normalized to percentage alteration from first sample (baseline). Next, differences between consecutive samples were calculated. This way we received adjusted cortisol changes for habituation, FRT, WCST, and closing assessment.

\subsection{5 $\quad$ Statistical analyses}

Differences in frequency of sinusoid-curve ratings between FRT and WCST were analyzed using Yates'-corrected chisquared test. To display averaged group differences over total test course, normalized Reference Index means and 95\% confidence intervals were calculated, highlighting significant differences where confidence intervals do not touch. To additionally investigate thermal dynamics over test quarters, mixed-design ANOVA was calculated, with quarter-sums of absolute Reference Index changes per arbitrary unit as dependent variable, test quarters as within- and test as betweenfactor. Bonferroni-adjusted post-hoc multiple comparison tests were calculated to compare differences between individual test quarters. Cortisol reactivity changes between FRT and WCST were analyzed by comparing cortisol- $\Delta$ group values via two-sided paired Student's $t$ test. All statistical tests were conducted in R using RStudio, with significance levels set to alpha $=0.05$. Welch-corrected Student's $t$ tests were used, and, in cases of violations of sphericity, HuynFeldt corrections were applied to repeated-measure ANOVA.

\section{3 | RESULTS}

\section{1 | Unexpected discovery: SocioBox recognition testing induces lasting social avoidance in mice}

To evaluate the social Y-maze test ${ }^{31,50}$ for suitability as routine sociability readout in our mouse behavioral test battery, we used mice which had previously undergone SocioBox ${ }^{30}$ experiments. By serendipity, we this way discovered that 4 weeks after passing through the SocioBox paradigm, these mice displayed social avoidance. This unexpected result was fully replicated in a second, independent cohort of former SocioBox completers (Figure 1A-C), leading to two first conclusions: (I) By chance, we may have developed a mouse model of social aversion priming/sociophobia, arising from a situation of inescapable social contacts. (II) The SocioBox test, even though superior to all other presently available social recognition tests and the first that successfully addresses multiple social contacts in parallel, will have to be treated as a final test in future behavioral test batteries (similar to eg, fear conditioning). 


\subsection{IRT as non-invasive tool to measure the vascular response to social cognitive performance in a "sociophobia" inducing setup}

To further explore the novel "mouse model of sociophobia", we employed IRT as a non-invasive method to continuously approximate experienced stress during these inescapable social contacts in the SocioBox (Figure 1D-I; video S1). Conveniently, IRT additionally provides monitoring of spatiotemporal dynamics, and thus location information needed for tracking. As expected, in the SocioBox recognition test, male mice spent significantly more time in the zone close to the stranger compared to already acquainted stimulus mice $\left(F_{(4,36)}=3.58 ; P=.015\right.$; Figure $\left.1 \mathrm{E}\right)$, while control mice (empty SocioBox $)$ did not exhibit any zone preference $\left(F_{(4,32)}=0.86\right.$; $P=.499$; Figure $1 \mathrm{~F})$. These findings were reproduced in female mice (SocioBox: $F_{(4,32)}=3.03 ; P=.032$; empty box: $\left.F_{(4,64)}=1.21 ; P=.314\right)$. Comparison of mean time spent with stranger versus all known mice yielded equivalent results $\left(\right.$ male $t_{(9)}=2.38 ; P=.021$; female: $t_{(8)}=2.20 ; P=.030$; Figure $1 \mathrm{G})$. Screening the obtained IRT readouts in a few males first, we observed that mice changed their temperature over time in the SocioBox in a typical way, namely displayed an increase in body and a decrease in tail temperature (video $\mathrm{S} 1$ ). We therefore introduced a novel descriptive measure, integrating an internal control (within-subject) aspect, the Centralization Index. This measure, likely approximating the experienced stress during the task, clearly demonstrates an increase in SocioBox mice versus controls for both males $\left(t_{(10.23)}=-4.44 ; P=.001\right)$ and females $\left(t_{(10.66)}=-2.27\right.$; $P=.045$; Figure $1 \mathrm{H})$. Importantly, enhanced movement and thus physical activity cannot account for this difference since control mice even had a tendency to move more than SocioBox performers $\left(t_{(36.18)}=1.90 ; P=.066\right)$. Interestingly, the Centralization Index correlated negatively with the time spent with the stranger in the SocioBox (Spearman's rho = $-0.51 ; P=.017$; Figure 1I), indicating that mice with a higher Centralization Index (likely reflecting their stress level) perform worse in this social recognition task.

\section{3 | Robust induction of social avoidance in the Y-maze sociability test following SocioBox recognition testing}

Around 4 weeks after SocioBox testing, mice were exposed to Y-maze sociability testing, including IRT (Figure 1J). While male control mice exhibited normal social preference $\left(F_{(2,16)}=7.88 ; P=.004\right.$; linear trend: $b=128.32 ; t_{(16)}=4.85$; $P=.0004$; quadratic trend: $\left.b=6.44 ; t_{(16)}=0.24 ; P=.811\right)$, the prior SocioBox performers displayed social avoidance behavior, similar to the discovery samples $\left(F_{(2,18)}=4.55\right.$;
$P=.025$; linear trend: $b=25.86 ; t_{(18)}=0.99 ; P=.334$; quadratic trend: $b=-92.48 ; t_{(18)}=-3.55 ; P=.002$; Figure $1 \mathrm{~K})$. Comparable effects were found for female mice in both control $\left(F_{(2,28)}=4.89 ; P=.015\right.$; linear trend: $b=57.70$; $t_{(28)}=3.80 ; P=.0007 ;$ quadratic trend: $b=0.23 ; t_{(28)}=0.02$; $P=.988)$ and post-SocioBox condition $\left(F_{(2,16)}=9.56\right.$ $P=.002$; linear trend: $b=44.67 ; t_{(20)}=2.16 ; P=.046$; quadratic trend: $b=-100.52 ; t_{(16)}=-4.87 ; P=.0002$; Figure $1 \mathrm{~L})$. These results, together with those of the two discovery samples, point to a robust induction of "sociophobia" by the SocioBox recognition test.

After Y-maze sociability testing, we controlled for potential differences in basic anxiety-related conduct. As a simple, non-invasive readout, spatial novelty-induced freezing in the fear-conditioning chamber (without shock) was evaluated. Importantly, neither male nor female SocioBox performers differed from empty box controls regarding duration of freezing (males: $F_{(2,36)}=0.09 ; P=.910$; females: $F_{(2,28)}=2.96$; $P=.068$, tendency in the opposite direction), excluding an "unspecific global fear behavior" underlying their "sociophobia" phenotype.

The next crucial question was whether we would see a correlation between stress, experienced in the SocioBox, as measured by the Centralization Index, and the degree of sociability evaluated 4 weeks later in the Y-maze. Indeed, a higher Centralization Index was associated with lower sociability (Spearman's rho $=-0.27 ; P=.049$ ) (Figure $1 \mathrm{M}$ ). Together, these results support our hypothesis that inescapable social encounters can induce sociophobia/social aversion in mice.

The Centralization Index during Y-maze sociability testing also tended to be increased in SocioBox mice $(\mathrm{M}=1.23$, $\mathrm{SD}=0.03)$ versus empty box controls $(\mathrm{M}=1.21, \mathrm{SD}=0.02$; both genders included; two-sided unpaired Student's $t$ test, $\left.t_{(34.47)}=-1.88, P=.068\right)$. Once again, these higher Centralization Index values could not be explained by higher physical activity; control animals traveled more than their SocioBox counterparts (both genders included; $t_{(36.07)}=2.81$; $P=.008)$.

\section{4 | Translational study: IRT as sensitive measure of the vascular response to social cognitive performance in humans}

In our mouse experiments, we unexpectedly discovered that stress experienced during an inescapable social encounter (SocioBox) likely acts as a "primer" of sociophobia/social aversion. We thus started an IRT study in men, investigating in a translational fashion, whether a simple social component in a cognitive task (face recognition) would already yield thermographic results differing from a "non-social" cognitive test (pattern recognition) (Figure 2A-C; Figure 
3A-I; Table 1; video S2). Of a total of $\mathrm{N}=228$ men screened for participation, $\mathrm{N}=111$ were tested, and $\mathrm{N}=103$ finally analyzed. Recruitment flow, study design and Face Recognition Test (FRT) are shown in Figure 2A-C.

All 103 subjects displayed high accuracy of face recognition in the prosopagnosia test (part of initial assessment; $\mathrm{M}=97.67 \%, \mathrm{SD}=6.45$ ) as prerequisite to perform the study. To ensure that subjects whose session started with FRT did not differ systematically from those with Wisconsin Card Sorting Test (WCST) first, we compared sociodemographic, psychopathological, and cognitive data between the two samples (Table 1). Since none of these variables showed any group differences, we combined both samples for the now following analyses of IRT readouts. First, we screened several facial IRT videos of participating subjects, allowing us to determine our regions of interest (ROI), namely nose (highly variable and seemingly responsive, as also described before $)^{15,18}$ and malar region of the cheek (obviously quite stable; compare video S2). We then calculated the Reference Index, in some analogy to the Centralization Index in mice, by dividing mean nose ROI temperature of each frame with its corresponding malar cheek ROI. Comparing the Reference Index course of all individuals during FRT, we noticed a sinusoid pattern predominating in most subjects (initial decrease, followed by increase) (Figure 3C). In contrast, only a minority of participants seemed to show such pattern during WCST (Figure 3D). To consolidate this first visual impression, independent raters estimated in a blinded fashion all individual thermal curves of both FRT and WCST to determine whether they resembled the characteristic sinusoid shape or not, with high interrater reliability (Cohen's kappa $=0.63$ between first two raters). Comparisons of pattern frequency (sinusoid versus not) yielded highly significant differences, with $77.7 \%$ of participants showing the sinusoid curve during FRT but only $30.1 \%$ during WCST $(O R=7.98$; Figure 3C-E). Interestingly, comparing participants with sinusoid-shaped Reference Index curve to those without revealed younger age and less time spent in the educational system together with higher scores in Social Phobia Scale (SPS) and Social Interaction Anxiety Scale (SIAS) ${ }^{38}$ as well as lower answer security in FRT (Table 2).

Presentation of the normalized Reference Index total course of all subjects for each test $(M \pm 95 \%$ confidence interval) illustrates the sinusoid pattern during FRT versus the

TA B LE 1 Sociodemographic, psychopathological, and cognitive data of test subjects performing FRT or WCST first

\begin{tabular}{|c|c|c|c|c|c|}
\hline & $\begin{array}{l}\text { Total sample } \\
\qquad \mathrm{N}=103\end{array}$ & $\begin{array}{l}\text { FRT first } \\
\text { N = 52 }\end{array}$ & $\begin{array}{l}\text { WCST first } \\
\qquad \mathbf{N}=\mathbf{5 1}\end{array}$ & $t / \chi^{2}$ & $P$ \\
\hline Neutral statement in FRT & $23(22.33 \%)$ & $12(23.08 \%)$ & $11(21.57 \%)$ & 0.03 & .854 \\
\hline Years of age & $24.58(3.34)$ & $24.76(3.49)$ & $24.40(3.20)$ & 0.55 & .584 \\
\hline Years of education & $16.83(2.67)$ & $17.02(2.86)$ & $16.65(2.51)$ & 0.65 & .520 \\
\hline BMI & $24.15(2.97)$ & $23.75(1.95)$ & $24.53(3.67)$ & -1.25 & .216 \\
\hline LPS-3 $(T)$ & $61.52(5.53)$ & $61.58(6.20)$ & $61.46(4.81)$ & 0.10 & .918 \\
\hline STAI state & $32.41(5.08)$ & $32.90(5.62)$ & $31.90(4.45)$ & 1.00 & .318 \\
\hline STAI trait & $32.85(6.94)$ & $33.79(7.47)$ & $31.90(6.27)$ & 1.39 & .168 \\
\hline SPS sum & $6.22(4.51)$ & $5.75(4.35)$ & $6.71(4.67)$ & -1.08 & .285 \\
\hline SIAS sum & $15.95(8.05)$ & $15.38(7.88)$ & $16.53(8.25)$ & -0.72 & .473 \\
\hline BSI sum $(T)$ & $45.81(9.07)$ & $46.21(9.58)$ & $45.39(8.59)$ & 0.46 & .649 \\
\hline NEO-Openness & $32.20(6.50)$ & $33.06(6.68)$ & $31.33(6.25)$ & 1.35 & .179 \\
\hline NEO-Conscientiousness & $32.31(7.21)$ & $33.38(7.09)$ & $31.22(7.23)$ & 1.54 & .127 \\
\hline NEO-Extraversion & $29.21(6.61)$ & $29.15(6.06)$ & $29.27(7.19)$ & -0.09 & .927 \\
\hline NEO-Agreeableness & $31.21(6.93)$ & $31.08(7.44)$ & $31.35(6.45)$ & -0.20 & .841 \\
\hline NEO-Neuroticism & $15.61(7.45)$ & $16.12(8.01)$ & $15.10(6.87)$ & 0.69 & .490 \\
\hline FRT error percentage & $42.28(12.04)$ & $41.06(12.58)$ & $43.53(11.46)$ & -1.04 & .300 \\
\hline FRT duration (s) & $969.12(64.88)$ & $975.24(68.86)$ & $962.74(60.48)$ & 0.98 & .332 \\
\hline FRT confidence & $3.40(0.52)$ & $3.44(0.47)$ & $3.36(0.57)$ & 0.78 & .437 \\
\hline WCST error percentage & $18.35(9.43)$ & $17.29(9.01)$ & $19.43(9.82)$ & -1.15 & .251 \\
\hline WCST duration (s) & $621.15(161.36)$ & $612.04(146.99)$ & $630.44(175.80)$ & -0.58 & .566 \\
\hline
\end{tabular}

Note: Data represent uncorrected means (SD) or N (\%). Student's $t$ tests and Pearson's chi-squared tests for independent comparisons were employed for analyses. Abbreviations: BMI, Body Mass Index; BSI, Brief Symptom Inventory; FRT, Face Recognition Test; LPS-3, Leistungsprüfsystem-3 (performance test system subtest-3); SIAS, Social Interaction Anxiety Scale; SPS, Social Phobia Scale; STAI, State-Trait Anxiety Inventory; WCST, Wisconsin Card Sorting Test. 
TA B L E 2 Sociodemographic, psychopathological, and cognitive data of subjects with/without sinusoid-shaped thermal curve during FRT

\begin{tabular}{|c|c|c|c|c|c|}
\hline & $\begin{array}{l}\text { Total sample } \\
\qquad N=103\end{array}$ & $\begin{array}{l}\text { No sinusoid } \\
\quad \mathbf{N}=\mathbf{2 3}\end{array}$ & $\begin{array}{c}\text { Sinusoid } \\
\mathbf{N}=\mathbf{8 0}\end{array}$ & $t / \chi^{2}$ & $P$ \\
\hline Neutral statement in FRT & $23(22.33 \%)$ & $5(21.74 \%)$ & $18(22.50 \%)$ & $<0.01$ & $>.999$ \\
\hline Presenting FRT first & $52(50.49 \%)$ & $12(52.17 \%)$ & $40(50.00 \%)$ & $<0.01$ & $>.999$ \\
\hline Starting at 09:00 AM & $56(54.37 \%)$ & $9(39.13 \%)$ & $47(58.75 \%)$ & 2.04 & .154 \\
\hline Years of age & $24.58(3.34)$ & $26.16(3.90)$ & $24.13(3.03)$ & 2.31 & .028 \\
\hline Years of education & $16.83(2.67)$ & $18.24(2.99)$ & $16.43(2.46)$ & 2.41 & .024 \\
\hline BMI & $24.15(2.97)$ & $24.92(4.72)$ & $23.92(2.21)$ & 0.92 & .366 \\
\hline LPS-3 $(T)$ & $61.52(5.53)$ & $62.41(4.24)$ & $61.26(5.84)$ & 1.04 & .302 \\
\hline STAI state & $32.41(5.08)$ & $31.57(6.27)$ & $32.65(4.70)$ & -0.77 & .447 \\
\hline STAI trait & $32.85(6.94)$ & $30.17(7.35)$ & $33.63(6.66)$ & -2.03 & .051 \\
\hline SPS sum & $6.22(4.51)$ & $4.09(3.41)$ & $6.84(4.62)$ & -3.13 & .003 \\
\hline SIAS sum & $15.95(8.05)$ & $12.91(5.85)$ & $16.83(8.40)$ & -2.54 & .014 \\
\hline BSI sum $(T)$ & $45.81(9.07)$ & $44.04(9.48)$ & $46.31(8.94)$ & 1.02 & .313 \\
\hline NEO-Openness & $32.20(6.50)$ & $33.00(5.90)$ & $31.98(6.68)$ & 0.71 & .481 \\
\hline NEO-Conscientiousness & $32.31(7.21)$ & $33.35(8.05)$ & $32.01(6.97)$ & 0.72 & .476 \\
\hline NEO-Extraversion & $29.21(6.61)$ & $30.57(6.40)$ & $28.83(6.66)$ & 1.14 & .262 \\
\hline NEO-Agreeableness & $31.21(6.93)$ & $31.13(6.77)$ & $31.24(7.02)$ & -0.07 & .948 \\
\hline NEO-Neuroticism & $15.61(7.45)$ & $14.26(8.25)$ & $16.00(7.21)$ & -0.92 & .367 \\
\hline FRT error percentage & $42.28(12.04)$ & $40.22(12.20)$ & $42.88(12.01)$ & -0.92 & .362 \\
\hline FRT duration (s) & $969.12(64.88)$ & $951.94(69.46)$ & $975.79(62.48)$ & -1.49 & .147 \\
\hline FRT confidence & $3.40(0.52)$ & $3.65(0.55)$ & $3.33(0.49)$ & 2.48 & .018 \\
\hline WCST error percentage & $18.35(9.43)$ & $16.96(6.72)$ & $18.75(10.08)$ & -1.00 & .323 \\
\hline WCST duration (s) & $621.15(161.36)$ & $594.41(139.00)$ & $636.83(169.32)$ & -1.23 & .225 \\
\hline
\end{tabular}

Note: Data represent uncorrected means (SD) or N (\%). Student's $t$ tests or Pearson's chi-squared tests for independent comparisons were employed for analyses. $\mathrm{P}$ values $<.05$ are indicated in bold.

Abbreviations: BMI, Body Mass Index; FRT, Face Recognition Test; WCST, Wisconsin Card Sorting Test; LPS-3, Leistungsprüfsystem-3 (performance test system subtest-3); STAI, State-Trait Anxiety Inventory; SPS, Social Phobia Scale; SIAS, Social Interaction Anxiety Scale; BSI, Brief Symptom Inventory. Because of the exploratory nature of the study, no $P$-value adjustments were conducted.

continuous decrease followed by a plateau during WCST (Figure 3F). Mean temperature changes over test quartiles as another readout of thermal dynamics were likewise found significantly different, with greater fluctuations in the second half of FRT (quartile $\times$ condition-interaction: $F_{(3,612)}=30.17$; $P=2.28 \times 10^{-15}$; Figure $\left.3 \mathrm{G}\right)$. Taken together, these data may point to an association of the sinusoid pattern with the emotional perception of the social task component.

Somewhat surprisingly, salivary cortisol alterations as "classical stress measures" did not differ during FRT and WCST $\left(t_{(101)}=-0.06 ; P=.952\right.$; Figure $\left.3 \mathrm{H}\right)$. This may question the validity of cortisol measurements for determining the specific stress caused by a social test component which can be sensitively detected by IRT. Nevertheless, cortisol reactivity during FRT correlated mildly positively with the time-adjusted integrals of Reference Index during FRT (Pearson correlation coefficient; $r=0.255 ; P=.026$; Figure 3I), indicating at least a slight "typical" stress reaction during FRT.
Contrary to our expectations, no differences were found upon presentation of negative feedback versus neutral statement during FRT, neither in Reference Index curve ratings $\left(\chi_{(1)}^{2}<0.01 ; P>.999\right)$, nor average Reference Index curve shape, nor post-feedback temperature dynamics (fluctuations during 3rd and 4th test quartile, repeated-measure ANOVA interaction effect: $\left.F_{(1,101)}=2.38 ; P=.126\right)$, nor any other variable (two-sided unpaired Student's $t$ tests; all $P>.05$ ). This suggests that the negative feedback did not have any relevant impact on these measures.

\section{4 | DISCUSSION}

The present translational study demonstrates that IRT can be applied as a convenient, easy-to-apply, non-invasive technology to sensitively and reliably assess physiological reactivity ("flushing") in social contexts in humans and mice. 
By implementing IRT within the SocioBox paradigm, we replicated and extended our previous findings that normal mice are able to easily recognize an unfamiliar stranger out of five stimulus mice. Strikingly, 4 weeks after performing this challenging social cognitive task, mice displayed abnormal social interaction in the Y-maze sociability test, namely distinct social avoidance. This unforeseen "sociophobia" following inescapable exposure to five conspecifics in the relatively narrow environment of the SocioBox was robustly reproduced several times in both genders. Control mice in the same narrow box without conspecifics ("empty") did not acquire this phenotype nor show any appreciable change in their basal anxiety behavior, as evaluated by spatial novelty-induced spontaneous freezing. Importantly, performance in the SocioBox as inducer of sociophobia was characterized by a higher overall Centralization Index, that is, higher temperature in central compared to peripheral body parts (tail), suggesting an increase in the experienced stress. Eye, body, or tail have previously been reported as stress-responsive IRT zones in mice. ${ }^{4}$ Considering body and tail temperature of mice in IRT simultaneously, as introduced here with the Centralization Index, seems to constitute a promising robust measure of autonomous responses/social stress. Interestingly, the Centralization Index during SocioBox correlated negatively with the time spent with the stranger mouse in the social recognition task as well as the succeeding sociability test, indicating that the degree of stress influences cognitive performance (SocioBox) as well as severity of social avoidance (Y-maze). Hence, the SocioBox paradigm may serve not only as a superior test of complex social recognition memory, ${ }^{30}$ but also as a reliable inducer of social avoidance, thereby delivering a novel non-invasive animal model of "sociophobia". ${ }^{51}$ As a consequence, the SocioBox test has to be used as a final test in a behavioral battery, similar to fear conditioning.

This unexpectedly strong relation between social cognition testing and IRT readouts in mice raised the obvious translational question whether the addition of a social component to a cognitive task would yield characteristic IRT data also in human individuals that differ from those obtained during a non-social test. In human IRT, thermo-patterns depend strongly on stimuli used and facial ROI targeted. ${ }^{9,16,20,29}$ We focused on the nose because of its high reactivity to social cues. $^{9,12,15-17,20}$ Since the introduction of the Centralization Index in mice had proven to be a reproducible, sensitive and widely environment-independent measure, we established a similar readout in human subjects. The Reference Index, again providing an "internal" (ie, within-subject) control by relating the responsive facial area (nose) to a rather temperature-stable zone (malar cheek) turned out to be a suitable tool to adjust for sources of IRT readout noise (eg, slight differences in ambient temperature, humidity, camera accuracy).., 29

Indeed, in the translational human study, we saw a characteristic sinusoid-shaped thermal curve with initial decrease in the majority of test subjects during the social FRT. In contrast, over the course of WCST, as non-social pattern recognition test, this typical curve was widely absent, with the temperature overall decreasing. However, not all participants responded with this characteristic social thermo-pattern, in the following referred to as "non-responders". Contrasting participants that exhibited the typical sinusoid-shaped thermo-pattern in FRT with the "non-responders" revealed interesting and plausible differences: younger age, less time spent in the educational system, higher scores in social phobia questionnaires (though still within the normal range), and less secure feedback-answers regarding their perceived own performance during FRT. Together, these differing items may point to lower experienced stress, ${ }^{15}$ that is, to a more "relaxed attitude" toward social test settings.

Whereas IRT analyses revealed differences between social/non-social tests, salivary cortisol levels, an established standard measure of the hypothalamus-pituitary-adrenal (HPA) stress response, did not. To exclude potential bias due to different starting times of our test sessions (9.00 AM or $11.00 \mathrm{Am})$, falling into the cortisol diurnal profile/awakening response, ${ }^{52-54}$ we employed normalized cortisol changes (delta values) between time points of sample collection. Comparing subject groups separated by starting time or order of test presentation (FRT or WCST first) did not reveal differences in delta cortisol values. These negative cortisol findings are in agreement with previous investigations on the potential of IRT in physical and social stress paradigms, compared to recognized stress markers. ${ }^{16}$ While thermal readouts in various facial regions were sensitive to stress-induced mood changes, conventional stress markers, such as cortisol, were not, ${ }^{16}$ suggesting a different origin (less HPA-related, more autonomic/catecholaminergic) and time course (fast versus delayed) of the experienced stress. Taken together, IRT seems to have a higher discriminative power for assessment of social cognition-related stress than cortisol.

Somewhat surprising, the fabricated negative feedback within FRT ("your performance is below average"), compared to the neutral statement ("you may now take a breaksecond part of test will start automatically") did not induce any measurable differences in thermograms. This may be due to a lower than expected socially threatening/embarrassing impact, the shortness of presentation (only $10 \mathrm{~s}$ each) ${ }^{13}$ with test-block 2 following immediately thereafter, or doubts of subjects regarding feedback authenticity. Finally, temperature alterations in connection with such ultra-brief negative feedback may belong to different underlying processes. ${ }^{20}$ Neither nose nor malar cheek may be optimal for exploring respective facial thermo-reactions.

In recent years, the potential of IRT as a valid research tool alongside traditional physiological approaches has been increasingly explored. ${ }^{9,15,17}$ However, IRT did not instantaneously turn out as a straightforward and easy-to-apply 
method. IRT is sensitive to numerous interfering factors, arising from environmental (eg, ambient temperature, humidity, room size, radiation) and individual sources (eg, gender, age, amount of brown adipose tissue, physical activity, food or substance intake). Further inconsistencies and reliability problems were caused by suboptimal study design, such as small or heterogeneous samples, artifacts due to manual ROI definition or quantification, or camera signal noise. ${ }^{9,29}$ Many studies did not sufficiently control for these methodological issues, leading to weak internal consistencies. ${ }^{9,29}$

In the present study, considerable effort was made to limit the impact of such interfering factors. As for the animal part, inbred mice were housed under controlled conditions and tested under standardized settings. In the human part, healthy male individuals with highly comparable sociodemographic characteristics were included. Large enough $(\mathrm{N}=45$ mice, $\mathrm{N}=103$ humans) test samples and standardized testing and recording procedures under constant ambient conditions were used as suggested by Fernández-Cuevas and colleagues. ${ }^{29}$ While various studies analyzed single or short series of images due to technological or memory-storage limitations, ${ }^{9}$ we used relatively long IRT video recordings (5-10 minutes for mice; $>15$ minutes per human participant and test) with high spatial and temporal resolution, and novel methods of data extraction and analysis. Centralization Index and Reference Index were introduced here as sensitive and widely environment-independent measures, providing internal (within-subject) control in the assessment of thermo-reactions in social contexts. Automated tracking and preprocessing algorithms delivered examiner-independent, objective and clean data extraction and organization, while imputations, smoothing, and winsorizing of data were conducted to reduce the impact of IRT camera inaccuracy, noise, and missing data.

Recently, IRT has also been employed for subjects diagnosed with mild posttraumatic stress disorder, Alzheimer's disease, or schizophrenia, ${ }^{55-57}$ underlining that psycholog$\mathrm{ical} /$ psychiatric research might profit from the contact-free, non-invasive IRT of freely moving and interacting subjects. In fact, deficits in social interaction/cognition of various origins are frequently seen in neuropsychiatry and often difficult to diagnose cross-sectionally. The current study on healthy individuals may stimulate future standardized social interaction testing using IRT in disease states, thereby opening new avenues for differential diagnostic approaches.

To summarize, based on a unique translational IRT study from mouse to man, we suggest that inclusion of a social component in a cognitive task specifically alters local body or face temperature, indicating a defined vascular response to this particular category of stress. These rather clear-cut findings were only possible on the ground of highly standardized and innovative experimental conditions, including IRT videos over an extended period to long-term monitor temperature alterations, unusually large, homogeneous subject samples, novel measures of internally (within-subject) controlled temperature over time, that is, Centralization and Reference Index and, finally, novel approaches to data acquisition, preprocessing, and analyses.

\section{ACKNOWLEDGEMENTS}

This study was supported by the Max Planck Society and the DFG Research Center for Nanoscale Microscopy and Molecular Physiology of the Brain (CNMPB).

\section{CONFLICT OF INTEREST}

The authors declare no competing financial or other interests.

\section{AUTHOR CONTRIBUTIONS}

Concept, study design and supervision: HE together with MM \& AS. Experimental design and interpretation: JS and FB together with HE \& AS. Data acquisition and analysis: JS, FB, MM, SM, AR, DKB, KS, HR, LW, FC, KB, AS \& HE. Drafting manuscript: HE together with JS, FB, MM \& AS. Drafting display items: JS, FB, MM \& HE. All authors read and approved the final version of the manuscript.

\section{DATA AVAILABILITY STATEMENT}

Full data availability will be provided. Accession codes will be available before publication.

\section{REFERENCES}

1. Darwin CR. The expression of the emotions in man and animals. London: John Murray; 1872.

2. Leary MR, Britt TW, Cutlip WD 2nd, Templeton JL. Social blushing. Psychol Bull. 1992;112:446-460.

3. Drummond PD. The effect of adrenergic blockade on blushing and facial flushing. Psychophysiology. 1997;34:163-168.

4. Gjendal K, Franco NH, Ottesen JL, Sorensen DB, Olsson IAS. Eye, body or tail? Thermography as a measure of stress in mice. Physiol Behav. 2018;196:135-143.

5. Meyer CW, Ootsuka Y, Romanovsky AA. Body temperature measurements for metabolic phenotyping in mice. Front Physiol. 2017;8:520.

6. Mufford JT, Paetkau MJ, Flood NJ, Regev-Shoshani G, Miller CC, Church JS. The development of a non-invasive behavioral model of thermal heat stress in laboratory mice (Mus musculus). J Neurosci Methods. 2016;268:189-195.

7. Herborn KA, Jerem P, Nager RG, McKeegan DEF, McCafferty DJ. Surface temperature elevated by chronic and intermittent stress. Physiol Behav. 2018;191:47-55.

8. Vianna DM, Carrive P. Changes in cutaneous and body temperature during and after conditioned fear to context in the rat. Eur $J$ Neurosci. 2005;21:2505-2512.

9. Ioannou S, Gallese V, Merla A. Thermal infrared imaging in psychophysiology: potentialities and limits. Psychophysiology. 2014;51:951-963.

10. Drummond PD. Facial flushing during provocation in women. Psychophysiology. 1999;36:325-332.

11. Drummond PD, Su D. The relationship between blushing propensity, social anxiety and facial blood flow during embarrassment. Cogn Emot. 2012;26:561-567. 
12. Esposito G, Nakazawa J, Ogawa S, et al. Baby, you light-up my face: culture-general physiological responses to infants and culture-specific cognitive judgements of adults. PLOS ONE. 2014;9:e106705.

13. Kistler A, Mariauzouls C, von Berlepsch K. Fingertip temperature as an indicator for sympathetic responses. Int J Psychophysiol. 1998;29:35-41.

14. Yoshihara K, Tanabe HC, Kawamichi H, et al. Neural correlates of fear-induced sympathetic response associated with the peripheral temperature change rate. NeuroImage. 2016;134:522-531.

15. Cardone D, Merla A. New Frontiers for Applications of Thermal Infrared Imaging Devices: Computational Psychophysiology in the Neurosciences. Sensors (Basel) 2017;17:E1042.

16. Engert V, Merla A, Grant JA, Cardone D, Tusche A, Singer T. Exploring the use of thermal infrared imaging in human stress research. PLoS ONE. 2014;9(3):e90782.

17. Kosonogov V, De Zorzi L, Honore J, et al. Facial thermal variations: a new marker of emotional arousal. PLOS ONE. 2017;12:e0183592.

18. Ermatinger FA, Brugger RK, Burkart JM. The use of infrared thermography to investigate emotions in common marmosets. Physiol Behav. 2019;211:112672.

19. Lahiri BB, Bagavathiappan S, Jayakumar T, Philip J. Medical applications of infrared thermography: a review. Infrared Phys Technol. 2012;55:221-235.

20. Ioannou S, Morris PH, Baker M, Reddy V, Gallese V. Seeing a blush on the visible and invisible spectrum: a functional thermal infrared imaging study. Front Hum Neurosci. 2017;11:525.

21. Ring EF, Ammer K. Infrared thermal imaging in medicine. Physiol Meas. 2012;33:R33- R46.

22. Garbey M, Sun N, Merla A, Pavlidis I. Contact-free measurement of cardiac pulse based on the analysis of thermal imagery. IEEE Trans Biomed Eng. 2007;54:1418-1426.

23. Murthy R, Pavlidis I. Noncontact measurement of breathing function. IEEE Eng Med Biol Mag. 2006;25:57-67.

24. Merla A, Di Donato L, Romani GL, Proietti M, Salsano F. Comparison of thermal infrared and laser doppler imaging in the assessment of cutaneous tissue perfusion in scleroderma patients and healthy controls. Int J Immunopathol Pharmacol. 2008;21:679-686.

25. Pavlidis I, Tsiamyrtzis P, Shastri D, et al. Fast by nature - how stress patterns define human experience and performance in dexterous tasks. Sci Rep. 2012;2.

26. Krzywicki AT, Berntson GG, O'Kane BL. A non-contact technique for measuring eccrine sweat gland activity using passive thermal imaging. Int J Psychophysiol. 2014;94:25-34.

27. Jarlier S, Grandjean D, Delplanque S, et al. Thermal Analysis of Facial Muscles Contractions. IEEE Transactions on Affective Computing. 2011;2:2-9.

28. Salazar-Lopez E, Dominguez E, Juarez Ramos V, et al. The mental and subjective skin: Emotion, empathy, feelings and thermography. Conscious Cogn. 2015;34:149-162.

29. Fernández-Cuevas I, Bouzas Marins JC, Arnáiz Lastras J, et al. Classification of factors influencing the use of infrared thermography in humans: A review. Infrared Phys Technol. 2015;71:28-55.

30. Krueger-Burg D, Winkler D, Mitkovski M, et al. The sociobox: a novel paradigm to assess complex social recognition in male mice. Front Behav Neurosci. 2016;10:151.

31. Brimberg L, Mader S, Jeganathan V, et al. Caspr2-reactive antibody cloned from a mother of an ASD child mediates an ASD-like phenotype in mice. Mol Psychiatry. 2016;21:1663-1671.
32. Sommer C, Straehle C, Kothe U, Hamprecht FA. Ilastik: Interactive learning and segmentation toolkit. IEEE. 2011;230-233.

33. Schindelin J, Arganda-Carreras I, Frise E, et al. Fiji: an opensource platform for biological-image analysis. Nat Methods. 2012;9:676-682.

34. Tinevez JY, Perry N, Schindelin J, et al. TrackMate: An open and extensible platform for single-particle tracking. Methods. 2017;115:80-90.

35. Wilcox RR. Winsorized Robust Measures. 2017; 1-2.

36. R Core Team. $R$ : A language and environment for statistical computing. Vienna: R Foundation for Statistical Computing; 2018.

37. Franke GH. BSI - Brief Symptom Inventory - deutsche Fassung. Göttingen: Beltz; 2000.

38. von Consbruch K, Stangier U, Heidenreich T. Skalen zur Sozialen Angststörung (SOZAS). Göttingen: Hogrefe; 2016.

39. Borkenau $\mathrm{P}$, Ostendorf F. NEO-Fünf-Faktoren Inventar (NEOFFI) nach Costa und McCrae. Göttingen: Hogrefe; 1993.

40. Grimm J. State-Trait Anxiety Inventory nach Spielberger. Deutsche Lang- und Kurzversion: Methodenforum der Universität Wien; 2009.

41. Heaton KH, Chelune GJ, Talley JL, Kay G, Curtiss G. Wisconsin Card Sorting Test: computer version 4 (WCST: CV4) research edn. San Antonio, TX: Harcourt Assessment; 2003.

42. Duchaine BC, Weidenfeld A. An evaluation of two commonly used tests of unfamiliar face recognition. Neuropsychologia. 2003;41:713-720.

43. Herzmann G, Danthiir V, Schacht A, Sommer W, Wilhelm O Toward a comprehensive test battery for face cognition: Assessment of the tasks. Behav Res Methods. 2008;40:840-857.

44. Warrington EK. Recognition memory test: Manual. Berkshire, UK: NFER-Nelson; 1984

45. Peirce JW. PsychoPy-Psychophysics software in Python. J Neurosci Methods. 2007;162:8-13.

46. Van Rossum G. Python 2.7.10 Language Reference. Samurai Media Limited; 2015.

47. Kulke L, Janßen L, Demel R, Schacht A. Validating the Goettingen Faces Database. Open Science Framework; 2017.

48. Horn W. L-P-S Leistungsprüfsystem. Göttingen: Hogrefe; 1983.

49. Mathis A, Mamidanna P, Cury KM, et al. DeepLabCut: markerless pose estimation of user-defined body parts with deep learning. Nat Neurosci. 2018;21:1281-1289.

50. Lai WS, Johnston RE. Individual recognition after fighting by golden hamsters: a new method. Physiol Behav. 2002;76:225-239.

51. Toth I, Neumann ID. Animal models of social avoidance and social fear. Cell Tissue Res. 2013;354:107-118.

52. Boehringer A, Tost H, Haddad L, et al. Neural correlates of the cortisol awakening response in humans. Neuropsychopharmacology. 2015;40:2278-2285.

53. Fries E, Dettenborn L, Kirschbaum C. The cortisol awakening response (CAR): facts and future directions. Int J Psychophysiol. 2009;72:67-73.

54. Clow A, Hucklebridge F, Stalder T, Evans P, Thorn L. The cortisol awakening response: More than a measure of HPA axis function. Neurosci Biobehav Rev. 2010;35:97-103.

55. Jian BL, Chen CL, Chu WL, Huang MW. The facial expression of schizophrenic patients applied with infrared thermal facial image sequence. BMC Psychiatry. 2017;17:229.

56. Di Giacinto A, Brunetti M, Sepede G, Ferretti A, Merla A. Thermal signature of fear conditioning in mild post traumatic stress disorder. Neuroscience. 2014;266:216-223. 
57. Perpetuini D, Cardone D, Chiarelli AM, et al. Autonomic impairment in Alzheimer's disease is revealed by complexity analysis of functional thermal imaging signals during cognitive tasks. Physiol Meas. 2019;40:034002.

\section{SUPPORTING INFORMATION}

Additional supporting information may be found online in the Supporting Information section.
How to cite this article: Seidel J, Bockhop F, Mitkovski M, et al. Vascular response to social cognitive performance measured by infrared thermography: A translational study from mouse to man. FASEB BioAdvances. 2020;2:18-32. https://doi. org/10.1096/fba.2019-00085 


\section{SUMMARY AND CONCLUSIONS}

As discussed and reported, we found strong associations between the amount of defined adverse environmental risk factors experienced before adulthood and violent aggression in patients suffering from schizophrenia. Environmental risk accumulation is further linked to aggression severity scores in two general population samples. Moreover, while we could not find epigenome-wide differences in DNA methylation, an examination of HDAC1 mRNA expression levels revealed significant group differences in relation to environmental risk load (high versus low). These results explicitly suggest that an accumulation of negative environmental experiences during childhood and adolescence has a substantial influence on aggression, which may be even more relevant with respect to the exhibition of aggressive behavior than a person's mental condition.

It is important to note that we do not report a direct, causal relation between these risk factors and aggression; neither living in a large city, migrating to another country, nor consuming cannabis once in life will transform anybody into a threat to others. However, these factors constitute valid predictors in the complex interplay of genetics and environmental experiences, with the ultimate outcome of aggressive phenotypes. Against this backdrop, the reported model lets us predict the likelihood of aggressive behavior manifestation and makes the identification of individuals at risk possible. Some of the reported risk factors are easily avoidable, such as consumption of certain substances, whereas negative effects of others may be possible to mitigate.

Future research should aim to replicate our findings and extend them to different contexts. It is yet not clear whether our model's large effects are specific to aggression phenotypes or if it has more general implications. Possibly, there are other, equally or even more important, factors not included in our current model. Similarly, some of the identified risk factors could be characterized more clearly, to improve its accuracy even further. For instance, concerning the risk factor Migration, our model does not distinguish between individuals seeking refuge and those who migrated due to educational or occupational 
reasons. Likewise, it might be beneficial to differentiate the risk factor Lifetime cannabis consumption further with respect to the severity of consumption or used cannabis type (Schoeler et al., 2016). However, the standard model revealed already large and solid effects over all examined groups.

Notably, model modifications for more specific research questions seem to be feasible and can easily be achieved. Currently, we are working on modifications of the standard model in order to apply it in different contexts. For example, we investigate the relationship between environmental risk and psychopathology in a group of young asylum seekers. In this approach, the standard model is modified to take the special, detrimental experiences commonly reported by persons fleeing their countries due to war or expulsion, into account. Within a second project, we assess the relationship of our model with the consumption of multiple illicit drugs (i.e., polytoxicomania). In both cases, the model of accumulated environmental risk exhibits promising results (in preparation).

Our work on the utilization of infrared thermography (IRT), on the other hand, resulted not only in the development of a new model of social avoidance ('social phobia') in mice but also improved our knowledge about the potentials of IRT recordings in both animal and human experiments. By introducing new thermal readouts as well as innovative routines of automated data acquisition, processing, and analysis we were able to deepen our insight into socially-induced temperature alterations over longer time periods. In this context, the many confounders that can undermine the reliability of thermal assessments have been considerably taken into account. The number of studies employing IRT in psychophysiological or neuroscientific context is growing fast, rendering reliable and state-of-the-art recording and quantification techniques more and more important.

IRT offers great prospects for research and applications, in particular when the usage of other measurement techniques is limited. This involves, besides others, settings where assessed individuals are not able to act in accordance with application protocols of other techniques or are not willing to cooperate. In such situations, IRT can increase our range of possibilities, due to its contact-free and non-invasive application mode. 
In particular, physiological research involving patients suffering from mental conditions can benefit greatly from IRT research. As shown by several recent studies, IRT can be successfully utilized in experiments with patient groups (Di Giacinto et al., 2014; Jian et al., 2017; Perpetuini et al., 2019). Additionally, IRT may prove itself to be a valuable supporting tool in diagnostical approaches of different psychiatric conditions exhibiting similar phenotypes. IRT may detect distinct thermal patterns and enable a more reliable discrimination of patients in regard to psychiatric diseases as well as individuals from the general population. Accordingly, IRT can potentially be used in therapeutical contexts, for example as a contact-free, more flexible measure of physiological states during the treatment of disorders that are associated with stress and arousal.

Taken together, this work aimed at improving our knowledge on behavioral and autonomous reactions to stressful experiences. The robust findings we reported along with our methodological suggestions have implications both for research and society. 


\section{BIBLIOGRAPHY}

Agid, O., Kohn, Y., \& Lerer, B. (2000). Environmental stress and psychiatric illness. Biomedicine \& Pharmacotherapy, 54(3), 135-141. doi: 10.1016/s0753-3322(00)89046-0 Aguilera, G. (2011). Hpa axis responsiveness to stress: implications for healthy aging. Experimental Gerontology, 46(2-3), 90-5. doi: 10.1016/j.exger.2010.08.023

Alpar, O., \& Krejcar, O. (2017). Quantization and equalization of pseudocolor images in hand thermography. In I. Rojas \& F. Ortuño (Eds.), Bioinformatics and biomedical engineering (pp. 397-407). Cham: Springer International Publishing.

Begemann, M., Grube, S., Papiol, S., Malzahn, D., Krampe, H., Ribbe, K., ... Ehrenreich, H. (2010). Modification of cognitive performance in schizophrenia by complexin 2 gene polymorphisms. Archives Of General Psychiatry, 67(9), 879-88. doi: 10.1001/ archgenpsychiatry.2010.107

Belbasis, L., Kohler, C. A., Stefanis, N., Stubbs, B., van Os, J., Vieta, E., ... Evangelou, E. (2018). Risk factors and peripheral biomarkers for schizophrenia spectrum disorders: an umbrella review of meta-analyses. Acta Psychiatrica Scandinavica, 137(2), 88-97. doi: $10.1111 /$ acps.12847

Benus, R. F., Koolhaas, J. M., \& van Oortmerssen, G. A. (1992). Individual strategies of aggressive and non-aggressive male mice in encounters with trained aggressive residents. Animal Behaviour, 43(4), 531-540. doi: 10.1016/s0003-3472(05)81013-9

Bhatnagar, S., Vining, C., Iyer, V., \& Kinni, V. (2006). Changes in hypothalamic-pituitaryadrenal function, body temperature, body weight and food intake with repeated social stress exposure in rats. Journal of Neuroendocrinology, 18(1), 13-24. doi: 10.1111/ j.1365-2826.2005.01375.x

American Psychological Association. (2013). Diagnostic and statistical manual of mental disorders (5th Revised ed.). Washington, DC: Author.

Schizophrenia Working Group of the Psychiatric Genomics Consortium. (2014). Biological insights from 108 schizophrenia-associated genetic loci. Nature, 511(7510), 421-7. doi: 10.1038/nature13595

Björkqvist, K. (2018). Gender differences in aggression. Current Opinion in Psychology, 19, 39-42. doi: 10.1016/j.copsyc.2017.03.030 
Blair, R. J. (2016). The neurobiology of impulsive aggression. Journal of Child and Adolescent Psychopharmacology, 26(1), 4-9. doi: 10.1089/cap.2015.0088

Boehringer, A., Tost, H., Haddad, L., Lederbogen, F., Wust, S., Schwarz, E., \& MeyerLindenberg, A. (2015). Neural correlates of the cortisol awakening response in humans. Neuropsychopharmacology, 40(9), 2278-85. doi: 10.1038/npp.2015.77

Brimberg, L., Mader, S., Jeganathan, V., Berlin, R., Coleman, T. R., Gregersen, P. K., ... Diamond, B. (2016). Caspr2-reactive antibody cloned from a mother of an asd child mediates an asd-like phenotype in mice. Molecular Psychiatry, 21(12), 1663-1671. doi: $10.1038 / \mathrm{mp} .2016 .165$

Brown, A. S. (2011). The environment and susceptibility to schizophrenia. Progress in Neurobiology, 93(1), 23-58. doi: 10.1016/j.pneurobio.2010.09.003

Brunner, H. G., Nelen, M., Breakefield, X. O., Ropers, H. H., \& van Oost, B. A. (1993). Abnormal behavior associated with a point mutation in the structural gene for monoamine oxidase a. Science, 262(5133), 578-80. doi: 10.1126/science.8211186

Byrd, A. L., \& Manuck, S. B. (2014). Maoa, childhood maltreatment, and antisocial behavior: meta-analysis of a gene-environment interaction. Biological Psychiatry, 75(1), 9-17. doi: 10.1016/j.biopsych.2013.05.004

Cardone, D., \& Merla, A. (2017). New frontiers for applications of thermal infrared imaging devices: Computational psychopshysiology in the neurosciences. Sensors, 17(5). doi: $10.3390 / \mathrm{s} 17051042$

Cardone, D., Pinti, P., \& Merla, A. (2015). Thermal infrared imaging-based computational psychophysiology for psychometrics. Computational and Mathematical Methods in Medicine, 2015, 984353. doi: 10.1155/2015/984353

Caspi, A., McClay, J., Moffitt, T. E., Mill, J., Martin, J., Craig, I. W., .. Poulton, R. (2002). Role of genotype in the cycle of violence in maltreated children. Science, 297(5582), 851-4. doi: 10.1126/science. 1072290

Darwin, C. R. (1872). The expression of the emotions in man and animals. London: John Murray.

de Boer, S. F. (2018). Animal models of excessive aggression: implications for human aggression and violence. Current Opinion in Psychology, 19, 81-87. doi: 10.1016/ j.copsyc.2017.04.006

Di Giacinto, A., Brunetti, M., Sepede, G., Ferretti, A., \& Merla, A. (2014). Thermal signature 
of fear conditioning in mild post traumatic stress disorder. Neuroscience, 266, 216-23. doi: 10.1016/j.neuroscience.2014.02.009

Drummond, P. D. (1999). Facial flushing during provocation in women. Psychophysiology, 36(3), 325-32. doi: 10.1017/s0048577299980344

Drummond, P. D., \& Su, D. (2012). The relationship between blushing propensity, social anxiety and facial blood flow during embarrassment. Cognition E Emotion, 26(3), 561-567. doi: 10.1080/02699931.2011.595775

Engert, V., Merla, A., Grant, J. A., Cardone, D., Tusche, A., \& Singer, T. (2014). Exploring the use of thermal infrared imaging in human stress research. Plos One, 9(3). doi: 10.1371/journal.pone.0090782

Ermatinger, F. A., Brugger, R. K., \& Burkart, J. M. (2019). The use of infrared thermography to investigate emotions in common marmosets. Physiology \& Behavior, 211, 112672. doi: 10.1016/j.physbeh.2019.112672

Fazel, S., Gulati, G., Linsell, L., Geddes, J. R., \& Grann, M. (2009). Schizophrenia and violence: systematic review and meta-analysis. PLOS Medicine, 6(8), e1000120. doi: 10.1371/journal.pmed.1000120

Fernández-Cuevas, I., Bouzas Marins, J. C., Arnáiz Lastras, J., Gómez Carmona, P. M., Piñonosa Cano, S., García-Concepción, M. n., \& Sillero-Quintana, M. (2015). Classification of factors influencing the use of infrared thermography in humans: A review. Infrared Physics \& Technology, 71, 28-55. doi: 10.1016/j.infrared.2015.02.007

Gjendal, K., Franco, N. H., Ottesen, J. L., Sorensen, D. B., \& Olsson, I. A. S. (2018). Eye, body or tail? thermography as a measure of stress in mice. Physiology \& Behavior, 196, 135-143. doi: 10.1016/j.physbeh.2018.08.022

Harro, J. (2018). Animals, anxiety, and anxiety disorders: How to measure anxiety in rodents and why. Behavioural Brain Research, 352, 81-93. doi: 10.1016/j.bbr.2017.10 .016

Heaton, K., Chelune, G., Talley, J., Kay, G., \& Curtiss, G. (2003). Wisconsin card sorting test: computer version 4 (wcst: Cv4) research edition. San Antonio, TX: Harcourt Assessment.

Hellhammer, D. H., Wust, S., \& Kudielka, B. M. (2009). Salivary cortisol as a biomarker in stress research. Psychoneuroendocrinology, 34(2), 163-71. doi: 10.1016/j.psyneuen .2008 .10 .026 
Herborn, K. A., Jerem, P., Nager, R. G., McKeegan, D. E. F., \& McCafferty, D. J. (2018). Surface temperature elevated by chronic and intermittent stress. Physiology E Behavior, 191, 47-55. doi: 10.1016/j.physbeh.2018.04.004

Herschel, W. (1800). Experiments on the refrangibility of the invisible rays of the sun. Philosophical Transactions of the Royal Society of London, 90, 284-292. doi: 10.1098/ rstl.1800.0015

Hollon, N. G., Burgeno, L. M., \& Phillips, P. E. (2015). Stress effects on the neural substrates of motivated behavior. Nature Neuroscience, 18(10), 1405-12. doi: 10.1038/nn.4114

Howes, O. D., \& Murray, R. M. (2014). Schizophrenia: an integrated sociodevelopmentalcognitive model. The Lancet, 383(9929), 1677-1687. doi: 10.1016/s0140-6736(13)62036 $-\mathrm{x}$

Ioannou, S., Gallese, V., \& Merla, A. (2014). Thermal infrared imaging in psychophysiology: potentialities and limits. Psychophysiology, 51(10), 951-63. doi: 10.1111/psyp.12243

Ioannou, S., Morris, P. H., Baker, M., Reddy, V., \& Gallese, V. (2017). Seeing a blush on the visible and invisible spectrum: A functional thermal infrared imaging study. Frontiers in Human Neuroscience, 11, 525. doi: 10.3389/fnhum.2017.00525

Jarlier, S., Grandjean, D., Delplanque, S., N’Diaye, K., Cayeux, I., Velazco, M. I., .. Scherer, K. R. (2011). Thermal analysis of facial muscles contractions. Ieee Transactions on Affective Computing, 2(1), 2-9. doi: 10.1109/T-Affc.2011.3

Jian, B. L., Chen, C. L., Chu, W. L., \& Huang, M. W. (2017). The facial expression of schizophrenic patients applied with infrared thermal facial image sequence. BMC Psychiatry, 17(1), 229. doi: 10.1186/s12888-017-1387-y

Joseph, D. N., \& Whirledge, S. (2017). Stress and the hpa axis: Balancing homeostasis and fertility. International Journal of Molecular Sciences, 18(10). doi: 10.3390/ijms18102224

Juruena, M. F. (2014). Early-life stress and hpa axis trigger recurrent adulthood depression. Epilepsy \& Behavior, 38, 148-59. doi: 10.1016/j.yebeh.2013.10.020

Keeney, A. J., Hogg, S., \& Marsden, C. A. (2001). Alterations in core body temperature, locomotor activity, and corticosterone following acute and repeated social defeat of male nmri mice. Physiology \& Behavior, 74(1-2), 177-184. doi: 10.1016/s0031-9384(01) 00541-8

Kim-Cohen, J., Caspi, A., Taylor, A., Williams, B., Newcombe, R., Craig, I. W., \& Moffitt, T. E. (2006). Maoa, maltreatment, and gene-environment interaction predicting 
children's mental health: new evidence and a meta-analysis. Molecular Psychiatry, 11(10), 903-913. doi: 10.1038/sj.mp.4001851

Kistler, A., Mariauzouls, C., Kuhr, C., Simmen, D., Maranta, C. A., Stratil, J., ... Suter, B. (1996). Acute sympathetic responses elicited by acupuncture are pain-related and non-specific. Complementary Medicine Research, 3(6), 269-278. doi: 10.1159/000210242

Kistler, A., Mariauzouls, C., \& von Berlepsch, K. (1998a). Fingertip temperature as an indicator for sympathetic responses. International Journal of Psychophysiology, 29(1), 35-41. doi: 10.1016/s0167-8760(97)00087-1

Kistler, A., Mariauzouls, C., \& von Berlepsch, K. (1998b). Saliva collection with salivettes $\mathbb{}$ is a sympathetic stimulus. Complementary Medicine Research, 5, 12-17.

Knapp, M., Mangalore, R., \& Simon, J. (2004). The global costs of schizophrenia. Schizophrenia Bulletin, 30(2), 279-93. doi: 10.1093/oxfordjournals.schbul.a007078

Kosonogov, V., De Zorzi, L., Honore, J., Martinez-Velazquez, E. S., Nandrino, J. L., Martinez-Selva, J. M., \& Sequeira, H. (2017). Facial thermal variations: A new marker of emotional arousal. Plos One, 12(9). doi: 10.1371/journal.pone.0183592

Krueger-Burg, D., Winkler, D., Mitkovski, M., Daher, F., Ronnenberg, A., Schluter, O. M., ... Ehrenreich, H. (2016). The sociobox: A novel paradigm to assess complex social recognition in male mice. Frontiers in Behavioral Neuroscience, 10, 151. doi: 10.3389/fnbeh.2016.00151

Kulke, L., Janßen, L., Demel, R., \& Schacht, A. (2017). Validating the goettingen faces database. Open Science Framework. doi: 10.17605/OSF.IO/4KNPF

Lahiri, B. B., Bagavathiappan, S., Jayakumar, T., \& Philip, J. (2012). Medical applications of infrared thermography: A review. Infrared Physics \& Technology, 55(4), 221-235. doi: 10.1016/j.infrared.2012.03.007

Lai, W. S., \& Johnston, R. E. (2002). Individual recognition after fighting by golden hamsters: a new method. Physiology E Behavior, 76(2), 225-39. doi: 10.1016/s0031 $-9384(02) 00721-7$

Martinez, R. C., Carvalho-Netto, E. F., Amaral, V. C., Nunes-de Souza, R. L., \& Canteras, N. S. (2008). Investigation of the hypothalamic defensive system in the mouse. Behavioural Brain Research, 192(2), 185-90. doi: 10.1016/j.bbr.2008.03.042

McEwen, B. S. (2007). Physiology and neurobiology of stress and adaptation: central role of the brain. Physiological Reviews, 87(3), 873-904. doi: 10.1152/physrev.00041.2006 
Meyer, C. W., Ootsuka, Y., \& Romanovsky, A. A. (2017). Body temperature measurements for metabolic phenotyping in mice. Frontiers in Physiology, 8, 520. doi: 10.3389/ fphys.2017.00520

Mick, E., McGough, J., Deutsch, C. K., Frazier, J. A., Kennedy, D., \& Goldberg, R. J. (2014). Genome-wide association study of proneness to anger. PLoS One, 9(1), e87257. doi: 10.1371/journal.pone.0087257

Miczek, K. A., Fish, E. W., De Bold, J. F., \& De Almeida, R. M. (2002). Social and neural determinants of aggressive behavior: pharmacotherapeutic targets at serotonin, dopamine and gamma-aminobutyric acid systems. Psychopharmacology, 163(3-4), 434-58. doi: 10.1007/s00213-002-1139-6

Moline, A., Galvez-Garcia, G., Fernandez-Gomez, J., De la Fuente, J., Iborra, O., Tornay, F., ... Gomez Milan, E. (2017). The pinocchio effect and the cold stress test: Lies and thermography. Psychophysiology, 54(11), 1621-1631. doi: 10.1111/psyp.12956

Mufford, J. T., Paetkau, M. J., Flood, N. J., Regev-Shoshani, G., Miller, C. C., \& Church, J. S. (2016). The development of a non-invasive behavioral model of thermal heat stress in laboratory mice (mus musculus). Journal of Neuroscience Methods, 268, 189-95. doi: 10.1016/j.jneumeth.2015.12.011

Nelson, R. J., \& Trainor, B. C. (2007). Neural mechanisms of aggression. Nature Reviews Neuroscience, 8(7), 536-46. doi: 10.1038/nrn2174

Palumbo, S., Mariotti, V., Iofrida, C., \& Pellegrini, S. (2018). Genes and aggressive behavior: Epigenetic mechanisms underlying individual susceptibility to aversive environments. Frontiers in Behavioral Neuroscience, 12, 117. doi: 10.3389/fnbeh.2018 .00117

Perpetuini, D., Cardone, D., Chiarelli, A. M., Filippini, C., Croce, P., Zappasodi, F., ... Merla, A. (2019). Autonomic impairment in alzheimer's disease is revealed by complexity analysis of functional thermal imaging signals during cognitive tasks. Physiological Measurement, 40(3), 034002. doi: 10.1088/1361-6579/ab057d

Piazza, P. V., \& Le Moal, M. (1998). The role of stress in drug self-administration. Trends in Pharmacological Sciences, 19(2), 67-74. doi: 10.1016/s0165-6147(97)01115-2

Raine, A. (2002). Biosocial studies of antisocial and violent behavior in children and adults: a review. Journal of Abnormal Child Psychology, 30(4), 311-26. doi: 10.1023/a: 1015754122318 
Ribbe, K., Friedrichs, H., Begemann, M., Grube, S., Papiol, S., Kastner, A., ... Ehrenreich, H. (2010). The cross-sectional gras sample: a comprehensive phenotypical data collection of schizophrenic patients. BMC Psychiatry, 10, 91. doi: 10.1186/1471-244X $-10-91$

Ring, E. F. (2010). Thermal imaging today and its relevance to diabetes. Journal of Diabetes Science and Technology, 4(4), 857-62. doi: 10.1177/193229681000400414

Ring, E. F., \& Ammer, K. (2012). Infrared thermal imaging in medicine. Physiological Measurement, 33(3), R33-46. doi: 10.1088/0967-3334/33/3/R33

Rogalski, A. (2002). Infrared detectors: an overview. Infrared Physics \& Technology, 43(3-5), 187-210. doi: 10.1016/s1350-4495(02)00140-8

Rogalski, A. (2012). History of infrared detectors. Opto-Electronics Review, 20(3), 279-308. doi: 10.2478/s11772-012-0037-7

Sariaslan, A., Lichtenstein, P., Larsson, H., \& Fazel, S. (2016). Triggers for violent criminality in patients with psychotic disorders. JAMA Psychiatry, 73(8), 796-803. doi: 10.1001/ jamapsychiatry.2016.1349

Schoeler, T., Petros, N., Di Forti, M., Klamerus, E., Foglia, E., Ajnakina, O., ... Bhattacharyya, S. (2016). Effects of continuation, frequency, and type of cannabis use on relapse in the first 2 years after onset of psychosis: an observational study. The Lancet Psychiatry, 3(10), 947-953. doi: 10.1016/s2215-0366(16)30188-2

Smith, S. M., \& Vale, W. W. (2006). The role of the hypothalamic-pituitary-adrenal axis in neuroendocrine responses to stress. Dialogues in Clinical Neuroscience, 8(4), 383-95.

Stepniak, B., Papiol, S., Hammer, C., Ramin, A., Everts, S., Hennig, L., ... Ehrenreich, H. (2014). Accumulated environmental risk determining age at schizophrenia onset: a deep phenotyping-based study. The Lancet Psychiatry, 1(6), 444-453. doi: 10.1016/s2215-0366(14)70379-7

Österman, K., Björkqvist, K., Lagerspetz, K. M. J., Kaukiainen, A., Landau, S. F., Frączek, A., \& Caprara, G. V. (1998). Cross-cultural evidence of female indirect aggression. Aggressive Behavior, 24(1), 1-8. doi: 10.1002/(sici)1098-2337(1998)24:1<1::aid-ab1>3.0 .co;2-r

Tattersall, G. J. (2016). Infrared thermography: A non-invasive window into thermal physiology. Comparative Biochemistry and Physiology Part A: Molecular E Integrative Physiology, 202, 78-98. doi: 10.1016/j.cbpa.2016.02.022 
Tiihonen, J., Rautiainen, M. R., Ollila, H. M., Repo-Tiihonen, E., Virkkunen, M., Palotie, A., ... Paunio, T. (2015). Genetic background of extreme violent behavior. Molecular Psychiatry, 20(6), 786-92. doi: 10.1038/mp.2014.130

Toth, I., \& Neumann, I. D. (2013). Animal models of social avoidance and social fear. Cell and Tissue Research, 354(1), 107-18. doi: 10.1007/s00441-013-1636-4

Ulrich-Lai, Y. M., \& Herman, J. P. (2009). Neural regulation of endocrine and autonomic stress responses. Nature Reviews Neuroscience, 10(6), 397-409. doi: 10.1038/nrn2647

Usamentiaga, R., Venegas, P., Guerediaga, J., Vega, L., Molleda, J., \& Bulnes, F. G. (2014). Infrared thermography for temperature measurement and non-destructive testing. Sensors, 14(7), 12305-48. doi: 10.3390/s140712305

van Os, J., \& Kapur, S. (2009). Schizophrenia. The Lancet, 374(9690), 635-645. doi: $10.1016 /$ s0140-6736(09)60995-8

Veroude, K., Zhang-James, Y., Fernandez-Castillo, N., Bakker, M. J., Cormand, B., \& Faraone, S. V. (2016). Genetics of aggressive behavior: An overview. American Journal of Medical Genetics Part B: Neuropsychiatric Genetics, 171B(1), 3-43. doi: 10.1002/ ajmg.b.32364

Vianna, D. M., \& Carrive, P. (2005). Changes in cutaneous and body temperature during and after conditioned fear to context in the rat. European Journal of Neuroscience, 21(9), 2505-12. doi: 10.1111/j.1460-9568.2005.04073.x

Waltes, R., Chiocchetti, A. G., \& Freitag, C. M. (2016). The neurobiological basis of human aggression: A review on genetic and epigenetic mechanisms. American Journal of Medical Genetics Part B: Neuropsychiatric Genetics, 171(5), 650-75. doi: 10.1002/ ajmg.b.32388

Won, E., \& Kim, Y.-K. (2016). Stress, the autonomic nervous system, and the immunekynurenine pathway in the etiology of depression. Current Neuropharmacology, 14(7), 665-673. doi: 10.2174/1570159x14666151208113006

Yaribeygi, H., Panahi, Y., Sahraei, H., Johnston, T. P., \& Sahebkar, A. (2017). The impact of stress on body function: A review. EXCLI Journal, 16, 1057-1072. doi: 10.17179/ excli2017-480

Yoshihara, K., Tanabe, H. C., Kawamichi, H., Koike, T., Yamazaki, M., Sudo, N., \& Sadato, N. (2016). Neural correlates of fear-induced sympathetic response associated with the peripheral temperature change rate. Neuroimage, 134, 522-531. 


\section{A Appendix}

\section{A.1 Accepted co-authorships}

\section{Co-author publication I}

Ursini, G., Punzi, G., Chen, Q., Marenco, S., Robinson, J. F., Porcelli, A., Hamilton, E. G., Mitjans, M., Maddalena, G., Begemann, M., Seidel, J., Yanamori, H., Jaffe, A. E., Berman, K. F., Egan, M. F., Straub, R. E., Colantuoni, C., Blasi, G., Hashimoto, R., Rujescu, D., Ehrenreich, H., Bertolino, A., Weinberger, D. R. (2018). Convergence of placenta biology and genetic risk for schizophrenia. Nature Medicine 24, 792-801.

\section{Personal contribution:}

I derived required phenotypical information of schizophrenia patients from the GRAS database to be used as an independent analysis sample. Further, I read and corrected the final version of the manuscript.

\section{Co-author publication II}

Pan, H.* , Oliveira, B. ${ }^{*}$, Saher, G. ', Dere, E., Tapken, D., Mitjans, M., Seidel, J., Wesolowski, J., Wakhloo, D., Klein-Schmidt, C., Ronnenberg, A., Schwabe, K., Trippe, R., Mätz-Rensing, K., Berghoff, S., Al-Krinawe, Y., Martens, H., Begemann, M., Stöcker, W., Kaup, F. J., Mischke, R., Boretius, S., Nave, K. A., Krauss, J. K., Hollmann, M., Lühder, F., Ehrenreich, H. (2019). Uncoupling the widespread occurrence of anti-NMDAR1 autoantibodies from neuropsychiatric disease in a novel autoimmune model. Molecular Psychiatry, 24, 14891501.

${ }^{*}$ Equally contributing authors 


\section{Personal contribution:}

I was responsible for the statistical analyses and graphical representation with respect to NMDA receptor antibody positivity and age in both animal and human (extended GRAS) samples. Additionally, I read, corrected, and approved the final version of the manuscript. 


\section{Convergence of placenta biology and genetic risk for schizophrenia}

Gianluca Ursini1,2,3, Giovanna Punzi 1,2, Qiang Chen', Stefano Marenco ${ }^{4,5}$, Joshua F. Robinson ${ }^{6}$, Annamaria Porcelli ${ }^{2}$, Emily G. Hamilton ${ }^{6}$, Marina Mitjans ${ }^{7}$, Giancarlo Maddalena ${ }^{2}$, Martin Begemann ${ }^{7}$ Jan Seidel ${ }^{7}$, Hidenaga Yanamori ${ }^{8}$, Andrew E. Jaffe ${ }^{1,9}$, Karen F. Berman ${ }^{4}$, Michael F. Egan ${ }^{10}$, Richard E. Straub', Carlo Colantuoni ${ }^{11,12,13}$, Giuseppe Blasi ${ }^{2}{ }^{2}$, Ryota Hashimoto ${ }^{8,14}$, Dan Rujescu ${ }^{15}$ Hannelore Ehrenreich ${ }^{7}$, Alessandro Bertolino ${ }^{2}$ and Daniel R. Weinberger 1,3,1,1,13,16*

Defining the environmental context in which genes enhance disease susceptibility can provide insight into the pathogenesis of (in this study, States, Italy, and Germany, the liability of schizophrenia explained by PRS is more than five times greater in the presence of early-life complications (ELCS) compared with their absence. Patients with ELC histories have significantly higher PRS than patients without ELC histories, which is confirmed in additional samples from Germany and Japan. The gene set composed of schizophrenia loci that interact with ELCs is highly expressed in placenta, is differentially expressed in placentae from complicated in comparison with normal pregnancies, and is differentially upregulated in placentae from male compared with female that do. Pathway analyses reveal that genes driving the PRS-ELC interaction are involved in cellular stress response; genes a subset of the most significant genetic variants associated with schizophrenia converge on a deveropmental trajectory sensitive to events that affect the placental response to stress, which may offer insights into sex biases and primary prevention.

chizophrenia is a complex disabling disorder that occurs in all putative neurodevelopmental origins ${ }^{11}$ and is also supported populations, with a lifetime morbidity risk of around $0.7-0.8 \%^{1}$ by many epidemiological studies. For example, the prevalence and a higher incidence in males compared with females. The of schizophrenia increases in offspring of mothers who were in high heritability of the disorder indicates a major role for genetic the second trimester during influenza epidemics; in a prospective variants in its etiology "; however, non-genetic influences involving study, maternal respiratory infection during pregnancy increased explaining at least part of the non-shared environmental contribu- $\quad$ fold $d^{5,12}$. More generally, schizophrenia has been associated with Animal studies have shown that exposure to environmental adverse events that occur during pregnancy and labor, at delivinsults in utero leads to altered response to stress postnatally, with ery, and early in neonatal life, ${ }^{3,2,13}$. Meta-analyses of this body of effects on brain development and behavior that are partly medi- literature have found that ELCs increase risk by 1.5- to 2 -fold ${ }^{13}$, a ated by gene expression changes in placenta also reveal that males are more $u$ lo background $\mathrm{d}^{13}$, which is consistent with preliminary evidence of An important role for the intra-uterine environment in a relationship between ELCs, hypoxia-related genes, and risk the etiology of schizophrenia is consistent with the disorder's for schizophrenia

"Lieber Institute for Brain Development, Johns Hopkins Medical Campus, Baltimore, MD, USA. ${ }^{2}$ Group of Psychiatric Neuroscience, Department of Basic Medical Science, Neuroscience and Sense Organs, Aldo Moro University, Bari, Italy. ${ }^{3}$ Departments of Psychiatry and Behavioral Sciences, Johns Hopkins Research Program, National Institutes of Health, Bethesda, MD, USA. FHuman Brain Collection Core, National Institute of Mental Health, Intramural Program, National Institutes of Health, Bethesda, MD, USA. 'Center for Reproductive Sciences, Department of Obstetrics, Gynecology and Reproductive Sciences, University of California, San Francisco, San Francisco, CA, USA. ${ }^{C}$ Clinical Neuroscience, Max Planck Institute of Experimental
Medicine, DFG Research Center for Nanoscale Microscopy and Molecular Physiology of the Brain, Göttingen, Germany. ${ }^{8 D}$ epartment of Psychiatry, Osake University Graduate School of Medicine, Osaka, Japan. ${ }^{\circ}$ Department of Mental Health, Johns Hopkins Bloomberg School of Public Health, Baltimore, MD, USA. ${ }^{1}$ Merck Research Laboratories, Merck and Co., Inc., Whitehouse Station, NJ, USA. "Department of Neurology, Johns Hopkins University for Genome Sciences, University of Maryland School of Medicine, Baltimore, MD, USA. ${ }^{4}$ Molecular Research Center for Children's Mental Development Luther University of Halle-Wittenberg, Halle, Germany. ${ }^{1} \mathrm{McK}$ Kusick Nathans Institute of Genetic Medicine, Johns Hopkins University School of Medicine,
Baltimore, MD, USA. "*-mail: drweinberger@llibd.org

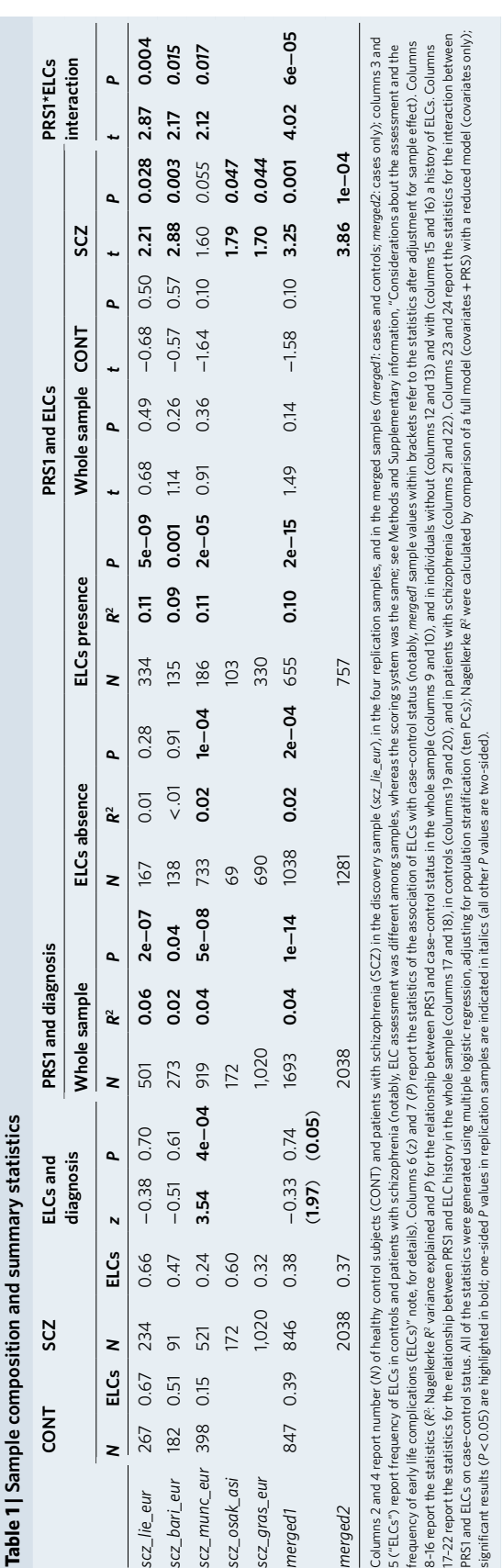

Genome-wide association studies (GWASs) indicate that genetic risk for schizophrenia across heterogeneous samples is conferred a a myriad of susceptibility genes ${ }^{17-1}$, indicating that the genetic architecture of the disorder is heterogeneous, consistent with polygenic mechanisms ${ }^{16,20}$. Although current GWASs are not designed to detect complex genetic and environmental heterogeneity ${ }^{16}$, we hypothesized that the most significant GWAS associations might achieve their statistical stat by converging on early developmental tively common among patients. In this article, we analyze the role played by the intra-uterine and perinatal environment in modulating the association of schizophrenia with genomic risk, with emphasis on the placental transcriptome.

Results

Interaction of polygenic risk score and early-life complications history on case-control status. We first investigated whether the intra-uterine and perinatal environment modulates the association
of schizophrenia with genomic risk. Specifically, we explored the interaction between genomic risk for schizophrenia and history of ELCs on the likelihood that a subject is a patient or a control, that is, case-control status. Genomic risk for schizophrenia was nificant alleles $\left(P<5 \times 10^{-8}\right.$. PRS1; single nucleotide polymorphisms (SNPs) in Supplementary Table 1 ${ }^{16}$, whereas ELC history was assessed with the McNeil-Sjöström scale ${ }^{22,23}$, defining a positive or negative ELC history based on the presence or absence of at least one potentially serious complication (that is, presence or in previous reports $5^{22,24,25}$. In the discovery sample of 501 individat

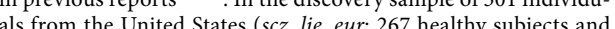
234 patients with schizophrenia, all Caucasian; see Table 1 and Supplementary Table 2 for sample information), the polygenic risk profile score constructed from alleles showing significant $(P<5 \times$ $10^{-8}$ ) association with schizophrenia (PRS1) in a meta-analysis of Psychiatric Genetic Consotium (PGC) GWAS datasets, after excluding the $s c z$ _lie_eur sample, was positively associ-
ated with case-control status $\left(N=501, t=5.347, P=2 \times 10^{-7}\right)$, so that patients had greater genetic risk compared with controls; PRS1 accounted for approximately $6 \%$ of risk prediction (Nagelkerkespseudo $R^{2}=0.060$; Fig. 1 a and SupplementaryTable3). In this sample, ELCs were not significantly different among healthy subjects and patients with schizophrenia $(z=-0.38$, $P=0.704)$. However, multiple logistic regression revealed a sigcontrol status $(t=287, P=0.004, \mathrm{Fig}, 1 \mathrm{~b}$ and Table 1$)$, results of the multiple regression also indicated that the ELC history was associated with schizophrenia when covarying for genetic risk score $(t=2.11, P=0.03)$, whereas PRS1 was not associated with schizophrenia when covarying for ELCs $(t=1.18, P=0.24)$. This result suggests that the association between genetic risk and ing the relationship between PRS1 and case-control status in the absence and in the presence of ELCs, we found that the liability of schizophrenia explained by the genetic risk score was highly significant in the context of ELCs ( $N=334$, Nagelkerke's pseudo $\left.R^{2}=0.112, t=5.97, P=5.02 \times 10^{-9}\right)$, but not in the absence of
them $\left(N=167\right.$ Nagelkerke's pseudo $R^{2}=0.008, t=1.07, P=0.28 ;$ Fig. 1b). We evaluated the same relationship in the context of Fig. 1b). We evaluated the same relationship in the context of
each severity level of ELCs; strikingly, whereas in the absence of potentially serious ELCs (weights 0-3) cases and controls were not different in PRS1, the two groups became clearly differentiated as the severity of ELCs increased (Fig. 1c). These results were not affected by the inclusion or exclusion of the top GWAS 


\section{ARTICLES}

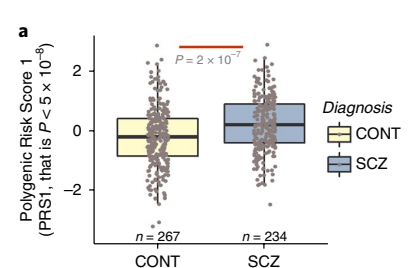

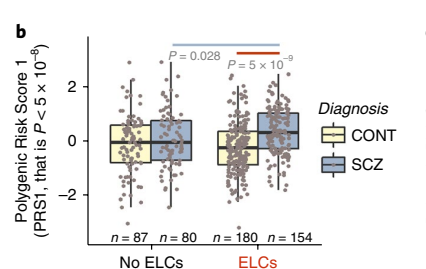

$n=87 n=80 \quad n=180, n=154$
\[ N \text { ELCS } \]

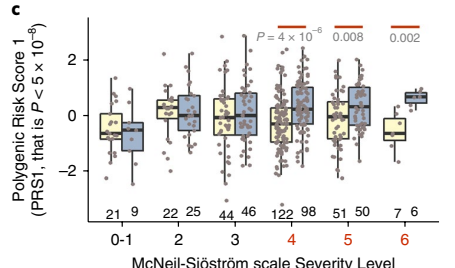

McNeil-Sï̈ström scale Severity Level

Fig. 1 | PRS1, ELC history, and schizophrenia in the scz_lie_eur sample ( $N=501)$. a, Association between genomic risk score (PRS1), constructed from alleles showing significant association with schizophrenia (with GWAS $P<5 \times 10^{-8}$ ), and case-control status. $\boldsymbol{b}$, Interaction between PRS1 and ELCS on in the presence of ELC history (right) c Relation between PRSI and case-control status in the context of ELCS with different severity levels severity scores $>4$ (in red) are considered harmful or relevant factors in fetal stress, whereas ELCs with severity scores of 0 . 1 , and 3 (in bect) are not All of the statistics were generated using multiple logistic regression, adjusting for population stratification (ten $\mathrm{PCS}$ ) (see also main text and Table 1 for detailed statistics). Boxplot centers depict median; lower and upper hinges correspond to 25th and 75th percentiles; whiskers extend from hinges to the Smallest and larger values no further than $1.5^{\star} \mid \mathrm{QR}$ from the 25 th and 75 th percentiles. Results in the other clinical samples are shown in Supplementary Figs. 3 and 4 .

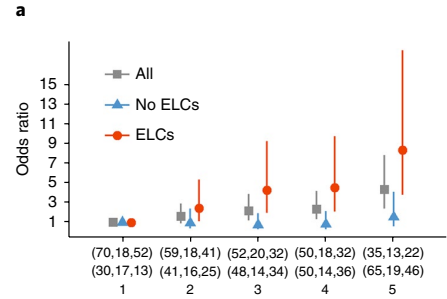

Quintile

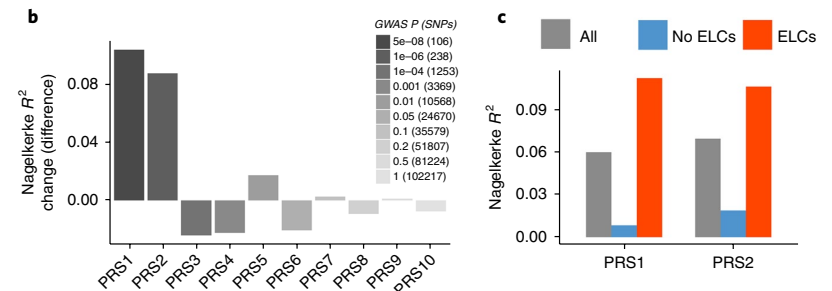

Fig. 2 | Liability of schizophrenia explained by genomic risk in the context of ELC history, in the scz_lie_eur sample $(N=501)$. a, OR for schizophreniz by PRS1 quintile and ELC history: the PRSs constructed from alleles showing significant association with schizophrenia (with GWAS $\left.P<5 \times 10^{-8}\right)$ were
converted to quintiles $(1=$ lowest PRS1, $5=$ highest PRSS), and the ORs were estimated for affected status for each quintile with reference to the lowest risk Converted to quintiles ( $(=l o w e s t ~ P R S T, 5=$ highest
quintilie, in the whole sample (gray square) and in the absence (blue triangles) and presence (orange dots) of ELCs. Bars correspond to $95 \%$ confidence
intervals. The presence of ELCs, in controls (first row) and in patients with schizophrenia (second row). b. Change of variance explained by genomic risk in the presence of ELCS compared with the absence of ELCS: proportion of variance (Nagelkerke R2) of schizophrenia risk was calculated, by comparison of a full model (covariates + PRS) with a reduced model (covariates only), in the presence and absence of ELC history, for each of the ten different PRSs constructed from alleles showing association with schizophrenia at different threshold of significance (GWAS $P$ value threshold and number of SNPs contributing to each W status (Nagelkerke R2, y axis) explained by PRSI and PRS2, in the whole sample (gray bars), in the absence of ELCS (blue bars), and in the presence of ELCS (orange bars). All of the statistics were generated using multiple logistic regression, adjusting for population stratification (ten PCs). Results in the other clinical samples are shown in Supplementary Figs. 3 and 4.

significant variant in the extended major histocompatibility complex (MHC) region (chr6: 25-34 Mb; Supplementary Table 4). levels, and we determined odds ratios (ORs) of being affected with schizophrenia associated with being in each PRS1 quintile, compared with the lowest PRS1 quintile. We also stratified our sample by ELC history to further represent the capacity of PRS1 to predict
risk for schizophrenia in the context of ELCS. The OR increased with risk for schizophrenia in the context of ELCs. The OR increased with ing the highest PRS1 quintile corresponded to an OR of $8.36(95 \%$ ing the higne interval [CI]: $\left.3.79-18.47, P=3.22 \times 10^{-8}\right)$ in the presence of ELCs, and only 1.55 ( $95 \%$ CI: $0.59-4.07, P=0.37$ ) in the absence

794
We further analyzed whether the interaction between genomic with schizophrenia at lesser statistical thresholds (that is, not GWAS significant). Interestingly, the ELC-dependent change in schizophrenia risk variance, explained by PRS, gradually decreased when (Fig 2b). Specifically only the first two scores, constructed from the alleles showing the strongest clinical association with schizophrenia (PRS1: $P<5 \times 10^{-8}$. PRS2: $P<1 \times 10^{-6}$ ), interact with ELCs on casecontrol status, and the variance in risk explained by them is much NATURE MEDICINE | VOL 24 | JUNE 2018 | 792 -801| www.nature.com/naturemedicine risk and ELCs was specific for the PRS constructed with GWAS
significant alleles (PRS1) or was also found with other PRS levels significant alleles (PRS1) or was also found with other PRS levels
(that is, PRS2-10) constructed from alleles showing association considering different PRSs constructed from variants showing asso-

\section{NATURE MEDICINE}

ARTICLES

higher in individuals with a history of ELCs, compared with those $\quad N=1,192)$ : another independent German sample of 1,020 patients without (Fig. 2b,c and Supplementary Figs. 1 and 2). The other scores, PRS3-10, do not show any interaction with ELCs, and the
variance explained by them is not influenced by a history of ELCs (Fig. 2b and Supplementary Figs. 1 and 2). This is consistent with the possibility that the latter scores, involving a greater number of putative susceptibility genes, capture a greater number of genetic risk variants acting in a simply cumulative way, whereas the aggre-
gate effect of the GWAS significant SNPs (PRS1) and the almost Gate effect of the GWAS significant SNPs (PRS1) and the almost GWAS significant SNPs (PRS2) is more conditioned on the history
of ELCs. These results raise the possibility that the reason PRS1 and PRS2 loci achieve their privileged statistical significance status in this heterogeneous clinical sample is because of this interaction. From another perspective, the data show that patients with a history of ELCs have greater PRS1 than patients without ELCs ( $N=234$ $t=2.21, P=0.028)$, whereas this relationship is not seen in healthy subjects $(N=267, t=-0.68, P=0.50)$. Maternal PRSs were availoffspring and were not associated with ELCs in their offering $(t=0.08, P=0.94$; Supplementary Table 5). Similarly, paternal PRSs were available only for a subsample of fathers $(N=136)$ and were also not significantly associated with ELCs in the offspring $(t=1.40$, $P=0.16$; Supplementary Table 5). These results suggest that the interaction between genomic 5 . and ELCs is mainly driven by the fetal genome and is relatively independent of gene-environment
interactions related to parental genomes per se. We sought to replicate our findings in several additional, independent samples. We first analyzed two case-control samples: an Italian sample of 273 subjects (scz_bari_eur) and a German sample of 919 subjects (scz_munc_eur) (see Table 1 and Supplementary Table 2 for sample information). As in the discovery sample, PRS (scz bari_eur: $N=273, R^{2}=0.02, t=2.11, P=0.036 ; s c z$ mamples

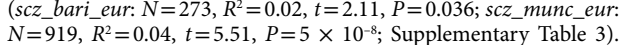
ELCs were not differentially distributed between healthy subjects and patients with schizophrenia in the $s c z$ bari_eur sample $(z=-0.51$, $P=0.61$ ), but there was a significant association of ELC history with schizophrenia in the larger $s c z_{-}$munc_eur sample $(z=3.54$, ples, PRS again showed a significant interaction with ELCs in pre$P \quad=0.017$; Table 1 and Suppleme Fig. $3 \mathrm{a}, \mathrm{b}$ ). When analyzing the relationship between PRS1 and case-control status in the context of ELCs, this PRS was once again associated with schizophrenia only in the presence of ELCs in the scz_bari_eur sample $(N=135, t=3.38, P=0.001)$, and not in their absence (N= the significant both in the absence $(N=733, t=3.88, P=0.0001)$ and in the presence of $\operatorname{ELCs}\left(N=186, t=4.45, P=2 \times 10^{-5}\right.$; Table 1 and Supplementary Fig. 3a,b). However, in both samples the variance of case-control status explained by PRS1 was much higher in individuals with a history of ELCs (scz_bari_eur: $R^{2}=0.09$; scz_munc_eur $R^{2}=0.11$ ) than in those without such history (scz_bari_eur
$R^{2}=0.0001$; $s c z$ munc eur: $R^{2}=0.02 ;$ Supplementary Fig $3 c$, d) again subjects who experienced ELCs who were in the upper quintile for PRS1 had the highest OR (scz_bari_eur: $\mathrm{OR}=6.67,95 \% \mathrm{CI}$ : $1.6-27.6, P=0.005 ; s c z$ munc_eur: OR $=14.17,95 \%$ CI: $4.0-49.9$,
$P=5.03 \times 10^{-6}$. Supplementary Fig. 3eff). These replication analyses also confirmed that PRSI was positively associated with ELC only in cases (scz_bari_eur: $N=91, \quad t=2.88, \quad P_{\text {onessided }}=0.003$; scz_munc_eur: $N=521, t=1.60, P_{\text {ones.sided }}=0.0547$ ), but not in con-
trols (scz bari_eur: $N=182, t=-0.57, P=0.57$; scz_munc $N=398, t=-1.64, P=0.10$; Table 1 and Supplementary Fig. 3a,b). Wefurther testedthe relationship between genomic risk for schizo-
phrenia and ELC history in two more samples of only patients (total NATURE MEDICINE | VOL 24 | JUNE 2018 | 792-801| www.nature.com/naturemedicie

with schizophrenia, namely, the Göttingen Research Association Japanese sample of 172 patients with schizophrenia (scz_osak_asi) (Table 1 and Supplementary Table 2). In regard to the latter, it should on oted that the PRS derived from the European Caucasian sample
of the recent GWAS study of schizophrenia has much less association with schizophrenia in Asian samples ${ }^{16}$, as would be expected is, the linke coltation between genotypes at nearby markers (that tions $^{26}$. However, because many of the alleles comprising the score likely monitor ancient haplotypes, an association with ELCs might still be expected. As in the three other samples, we again found that PRSI was associated with ELCs in both samples of patients with

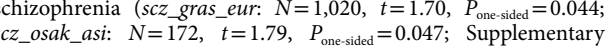
Fig. 4), so that patients with a history of complications had higher

ned analyses in merged samples of cases and con-

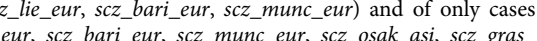
(u), after normalization of $\mathrm{PRSs}$ in each population. In these merged control status ( $N=1,693, t=4,02, P=6.18 \times 10^{-5}$, Sups on caseFig. 5) and the relationship between PRS1 and ELCs in patients with schizophrenia $(N=2,038, t=3.86, P=0.0001$ - Supplementary Figs. 5 and 6). Also, in the merged samples, only PRS1 and PRS2 ateract with ELCs on case-control status, and only PRS1 and PRS2
re positively associated with ELCs in patients with schizophrenia (Supplementary Figs. 5 and 6). Again, the positive association the trend was actually negative $(P=0.10$; Table 1$)$, which is, where ible with a pattern of a gene-environment interaction Sensitivity analyses with sex, age, paternal and maternal ages, maternal stress, socioeconomic status, and substance use as covariates and elated interaction terms (in addition to genetic principal compo(Shes) confirmed the same results (Supplementary Tables $6-8$ ). hypothesis that these top PRSs are rendevant for the support the esis of schizophrenia particularly in the context of ELCs, whereas other PRSs (that is, PRS3-10) may capture polygenic mechanisms

Expression of schizophrenia risk-associated genes in placenta. Even though several recent studies show preferential regulation hip between the PRSs and ELCs that we found in five independent clinical samples from diverse ancestries points to the intra-tterine context as a likely place where some risk genes for schizophrenia and environmental adversity intersect, with implications not limted to the brain. Because PRS1 and PRS2 risk SNPs are associ(Supplth expression of nearby genes across many different tissues SNPs for eQTLs across different tissues" note), we tested whether the genes mapping to the loci showing the strongest association PRS2 genes; Fig. $2 b$ and Supplementary Table 9) were more highly expressed in the placenta, compared with randomly selected genes contributing to PRSs constructed from alleles showing association which do not show an interaction with ELCs (that is PRS3 -10$)$. We analyzed RNA sequencing data from placental tissue generated in the Epigenome Roadmap Project (GSE16368), and found genes), compared with same size set of genes randomly selected
from PRS3-10 genes ( $N=18,029$ genes), in multiple placental tissue ○ 2018 Nature America lnc part of Springer Nature. All rights reserved 
Table 2 | Differential expression of schizophrenia risk genes in placentae from complicated pregnancies Dataset Condition

Tissue

GSE24129 Pre-eclampsia

GSE24129

GSE35574

GSE35574

GSE10588

GSE12216

GSE40182

GSE12767

GSE25861

SE65271

GSE28619

GSE4804

GSE27411

GSE42955

GSE3586

GSE4172

GSE4483

GE26420

GSE64699 IUGR
$P$ value of $\chi^{2}$ tes

$\begin{array}{ll}3.5 \mathrm{e}-04 & 0.002 \\ 0.019 & 0.0007\end{array}$

$\begin{array}{ll}0.019 & 0.0007 \\ 0.04 & 0.062\end{array}$

0.04

0.007

0.003

0.01

0.01
0.009
0.003

0.003

0.006

0.005

0.136

0.285

0.453

0.172

0.283

0.246

0.470

0.263
0.109

0.03

0.002

0.03

0.01

0.04
0.005

0.04

0.002

0.10
0.20
0.45

0.20 (oppos

0.34
0.40

0.42

0.37

3.5e-04 0.002

\begin{tabular}{ll}
$\chi^{2}$ \\
\hline palue & 7.93
\end{tabular}

0.10 (opposite direction) $\quad 1.63$
0.002 (opposite direction) $\quad 8.01$
0.37

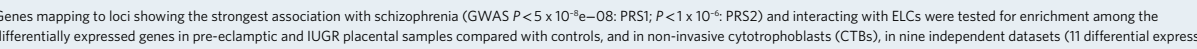

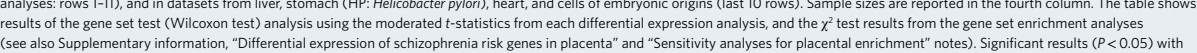

compartments: amnion $\left(N=4\right.$ samples, $\left.P=1 \times 10^{-4}\right)$, basal plate impaired migration of extravillous trophoblasts and impaired spi$\begin{array}{ll}\left(N=4, P=1 \times 10^{-4}\right) \text {, chorion }\left(N=4, P=3 \times 10^{-4}\right) \text { villi }(N=4, P=1 \times & \text { ral artery remodeling } 23,3,3,35 \\ \left.10^{-5}\right) \text {, and often associated with an altered }\end{array}$ $P=3 \times 10^{-5}$; third trimester: $N=2, P=1.6 \times 10^{-6}$. Supplementary IUGR are multifactorial syndromes and indeed are frequently Table 10). These results indicate that, as predicted, genes mapping linked with many other ELCs detected in our samples, includGWAS significant loci that interact with ELCs are more abun- ing diabetes, obesity, alcohol use, vaginal bleeding, maternal dantly expressed in placenta than are genes in the other GWAS which do not interact with ELCs.

Differential expression of schizophrenia risk-associated genes smoking, preterm birth and other adverse birth outcomes, and perinatal morbidity 38 -41. They are themselves classic severe ELCs
(severity level $\geq 4$ in the McNeil-Sjöström scale) that have been (severty level $\geq 4$ in the McNol-sjostrom scale) that have been primary affected cells have been isolated and studied ex vivivi ${ }^{13,2,2,43}$ In analyzing eight publicly available datasets, we consistently detected enrichment of the PRS1 and PRS2 genes (Table 2 and Supplementary Table 9) among the genes differentially expressed in the fetal portion pre-eclampsia and IUGR, specifically in pre-eclamptic (GSE24129:
$P=3.5 \times 10^{-4}$ : GSE3 35574:0.04: GSE10588:0.003: GSE25906: 0.02) and IUGR (GSE24129: $P=0.019$; GSE35574: 0.007; GSE12216: $0.01)$ chorionic tissue from term placentae, in pre-eclamptic cytotrophoblast (GSE40182: $P=0.009$ ) and first trimester chorionic villi (GSE12767: $P=0.003$ ), and in microvascular endothelium from $(U G R / p r e-e c l a m p t i c$ pregnancies (GSE25861: $P=0.006$ ). We observed that PRS1 and PRS2 genes tend to be upregulated (posiand IUGR, ompared with phental controls (Supplementary information, see "Differential expression of schizophrenia risk genes in placenta" note). Because the PRS1 and PRS2 genes were among the highly expressed placental genes, we then performed a
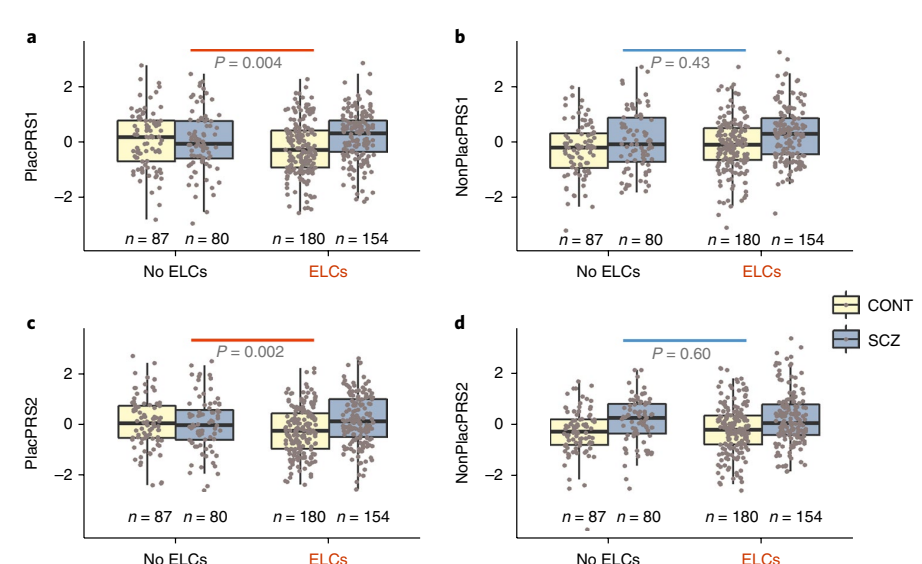

官 $\mathrm{coNT}$
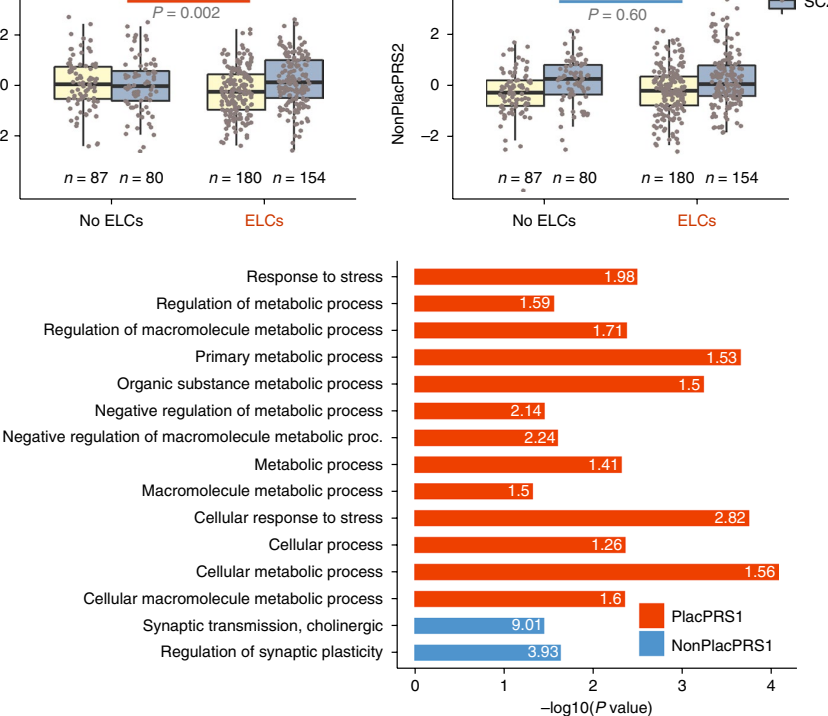

Fig. 3 | Placental and non-placental genomic risk for schizophrenia. a-d, Using GWAS SNPs marking loci containing genes highly and differentially expressed in pre-eclamptic/IUGR placental samples, we created new PRSs (PlacPRSs) and compared their interaction with ELCS with PRSs derived from the SNPs marking the remaining GWAS significant loci (NonPlacPRSS). The figure shows the interaction between PRSs and ELCs on case-control status in the US discovery sample (sc_lie_eur: N=501): shown are PlacPRS1 (a), NonPlacPRS1 (b), PlacPRS2 (c), and NonPlacPRS2 (d) of controls (CONT) and patients with schizophrenia (SCZ), in the absence of ELCS (left side of each boxplot) and in the presence of ELC history (right). Results in the other clinical samples are shown in Supplementary Figs. 7-9. Al the statistics were generated using multiple logistic regression, adjusting tor population stratification
(ten PCs); the P $P$ values refer to the significance of the interaction of ELCS with PlacPRSs (orange lines, ac) and with Non PlacPRSs (blue lines, bd): boxplot centers depict median; lower and upper hinges correspond to 25 th and 75 th percentiles; whiskers extend from hinges to smallest and larger values no further than $1.5 * \mid 5^{\star}$ from the 25th and 75 th percentiles. e, Biological processes Gene Ontology terms enriched for PlacPRS1 genes ( $N=130$, orange bars)
and NonPlacPRS1 genes ( $N=707$, blue bars) (see also Supplementary Tables $9,15-18$ and Supplementary figs. $10-20$ ): bars depict negative logarithm of the $P$ values, and white numbers within bars correspond to fold enrichment.

sensitivity analysis controlling for average gene expression level sensitivity analysis controlling for average gene expressio
and the results were consistent (Supplementary Table 11).

differentially expressed in the pathological compared with of PRS1 and PRS2 genes in complicated placentae might be a Supplementary information, see "Sensitivity analyses for placennonspecific response to pathology or stress in adult or fetal tissue. We therefore performed the same differential expression analyses on datasets from tissues with diseases likely unreHelicobacter pylori (HP) gastritis infection (GSE27411), and dilatative cardiomyopathy (GSE42955, GSE4172), as well as in datasets from embryonal cells (GSE4483, GSE26420, GSE64699). The PRS1 and PRS2 genes were not enriched among the genes tal enrichment" note).

Taken together, these results converge on the conclusion that schizophrenia GWAS significant risk-associated genes that interact
with ELCs are highly expressed in the placenta during early life and dynamically modulated in the placenta during biological stress, as reflected in their differential expression in placentae from complicated pregnancies, and that these associations are relatively placental specific

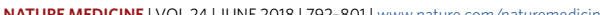



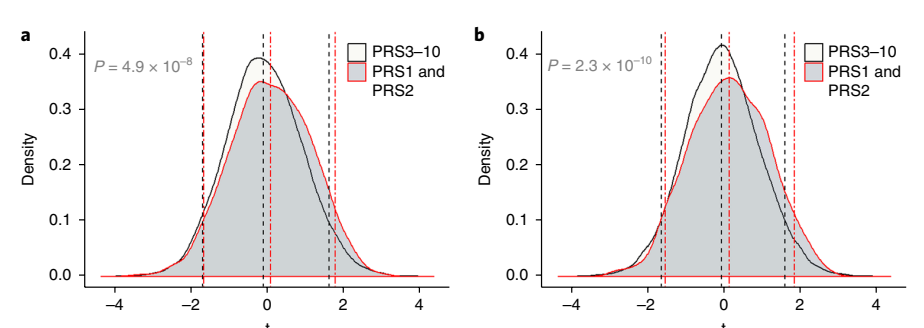

Fig. 4 | Upregulation of schizophrenia risk genes in male compared with female placentae. a,b, PRS1 and PRS2 genes were tested for enrichment among the differentially expressed genes in placentae from male compared with female offspring in two placental datasets (a: $G S E 35574: N=40,17$ females
and 23 males; $\mathbf{b}: G S E 25906: N=37,16$ females and 21 males). Shown are the density plots of the t-statistics, from the differential expression analysis, of PRS1 and PRS2 genes (dark gray area), and of all of the other genes in PRSs (PRS3-10, ivory area) constructed from lesser statistical thresholds (negative $t$-statistics $=$ more expressed in females; positive $t$-statistics = more expressed in males; $P_{\text {one sised }}$ from the Wilcoxon 'geneSet Test' statistics at the top the graphic). Dotted ines depict $95 \%$ (onfidence intervals and median of the moderated $t$-statistics from
regression) of PRS1 and PRS2 genes (red double-dotted lines) and of PRS3-10 genes (black dotted lines)

Genes highly and differentially expressed in placenta drive the interaction between polygenic risk scores and early-life complithe placenta of genes in schizophrenia GWAS significant loci pro-
vides circumstantial evidence that the interaction of these loci with ELCs on risk for schizophrenia arises at least in part because of primary effects in the placenta. To achieve a more direct test of this possibility, we created new PRSs based on the GWAS SNPs marking loci-containing genes highly expressed in normal placentae
and dynamically modulated in placentae from complicated pre and dynamics (Table 2 and Supplementary Table 9), and compated preginteraction with ELCs with PRSs derived from the SNPs marking the remaining GWAS significant loci, first in our discovery sample (scz_lie_eur; Fig. 3a-d). The PRSs from the former group significantly interact with ELCs in increasing risk for schizophrenia (PlacPRS1 (PRS1 "placental" subset based on 56 SNPs): $t=2.86$, $P=0.004$; PlacPRS2 (PRS2 "placental" subset based on 112 SNPs)
$t=3.10, P=0.002$. Fig. $t=3.10, P=0.002$; Fig. 3a,c), whereas those from the latter do not
(NonPlacPRS1 (PRS1 "non-placental" subset based on 49 SNPs): $t=0.78, P=0.43$; NonPlacPRS2 (PRS2 "non-placental" subset based on 125 SNPs): $t=-0.53, P=0.60$; Fig. 3b,d). Similar results were found in both other case-control samples (Supplementary
Figs. 7 and 8 ). Analyses on the merged samples of cases and conFigs. 7 and 8). Analyses on the merged samples of cases and con-
trols (scz_lie_eur, scz_bari_eur, scz_munc_eur: $N=1,693)$ controls (scz_lie_eur, scz_bari_eur, scz_munc_eur: $N=1,693)$ con-
firm these results; PlacPRS1 $(t=3.19, P=0.0014)$ and PlacPRS2 $(t=3.28, P=0.0011)$ significantly interact wh control status, where 9)
(Supplementary Fig.

To verify the specificity of these interactions to placenta gene expression, we calculated PRSs based on the genes highly expressed in various adult and fetal tissues/embryonic cells, and differentiall expressed in these organs during pathogical/stress compared with for the computation of PlacPRSs and NonPlacPRSs (Supplementary information, see "Sensitivity analyses for placental enrichment" note). We also calculated brain PRSs, based on SNPs in PRS1 and PRS2 loci associated with methylation quantitative trait loci in adult brain $^{29}$ and with chromatin interaction in fetal brain $^{27}$. In all of these sensitivity analyses, the PRSs comprising SNPs marking loci having
genes highly expressed in these diverse adult and fetal tissues, and dynamically regulated in adult and fetal brain, as in the pathology of heart, liver, and stomach, and in pathological cells of embryonic origins, do not significantly interact with ELCs on risk for schizo-
phrenia (all $P>0.16$ after false discovery rate correction; results are

in Supplementary Tables 12-14), whereas only the SNPs mapping to (ifferentially expressed in placenta do. Biological insights about placental-enriched genes associated
with ELCs. Both PlacPRS1 and PlacPRS2 genes are significantly enriched for many pathways related to metabolic and cellular stress and hypoxia, particularly to "unfolded protein response", "mito10 and 11: Supplementand "HIF1 $\alpha$ signaling" (Supplementary Figs. pathway enrichment could be obtained from the remaining PRS1 and PRS2 genes (NopPlacPRS1 and NonPlacPRS2) as well as from the whole PRS1 and PRS2 gene sets, consistent with the andogously negative results of the original analysis of the GWAS significant oci' (Supplementary information, see "Pathway, functional and coexpression analyses" note). Notably, the pathways (Supplementary
Figs. 10 and 11 ; Supplementary Table 15), biological functions and processes (Fig. 3e; Supplementary Figs. 12-14; Supplementary 15 and 16; Supplementary Table 17) implicated in PlacPRS genes are virtually orthogonal to those highlighted in other analyses of schizophrenia loci, such as synaptic function, calcium signaling, fragile $\mathrm{X}$-associated proteins, and chromatin remodeling ${ }^{16}$.

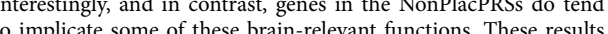
suggest that the loci containing the schizophrenia-associated genes dynamically modulated and most enriched in the placenta contribute to schizophrenia risk at least in part by influencing the fetal/ placental response to stress (Supplementary Figs. 17-19), as exem作 Fig 18 and Supplementary genes in PlacPRS2 (Supplementary nalyses reveal that the PlacPRS1 and PlacPRS2 genes nificantly co-expressed with immune response genes, in contrast sized gene sets of non-schizophrenia-associated genes in the same datasets (Supplementary Tables 19 and 20; Supplementary Fig. , Supplementary information, see "Pathway, functional and coThe sugrion analyses" note).

The suggestion of a distinct and orthogonal biology for the placental component of genomic risk raises the question whether genetic prediction might be enhanced by deconstructing genomic
risk into discrete subcompartments that represent alternate risk biologies. An exploratory analysis revealed that the agreregate effect on prediction accuracy of the SNPs contributing to PRS3-10 NATURE MEDICINE | VOL 24| JUNE 2018 | 792-801 | www.nature.com/naturemedicine (which include PRS1 loci) is higher when separating the contribution of PRS1 (Supplementary Fig. 21; Supplementary information,
see "Variance of schizophrenia liability explained by decomposed" see "Variance of schizophrenia liability explained by decomposed
PRSs" note for details). This is particularly true in the context of a history of ELCs, for each PRS. These results suggest that decomposing PRSs based on early environmental exposure and placental genetic risk may increase the prediction accuracy of genetic variation for schizophrenia.

Sex-specific analyses. The interaction between ELCS and genetic
risk for schizophrenia is consistent with a bedy of literature pointing to the placenta as a mediator of stress effects on the developing brain ${ }^{-9}$. Animal studies also have shown that the outcomes of altered placental functioning on neurodevelopment are substantially sex-specific, with males more vulnerable than females to pre-
natal adversity ${ }^{\text {s, }}$. Epidemiological studies of schizophrenia suggest natal adversity ${ }^{8,9}$. Epidemiological studies of schizophenia suggest
that incidence is higher in males than in females ${ }^{2}, 6,4,7$, despite the that incidence is higher in males than in females $2,4,4,7$, despite the
prevalence being similar across sexes, 1,2 , likely because of high prevalence being similar across sexes ${ }^{2,}$, likely because of higher
mortality in males ${ }^{48}$. Consistently, most evidence suggests that
males have an earlier age of onset of schizophrenia s $^{4,50}$, which is also a predictor of a worse prognosis $s^{9-51}$, and is plausibly linked with a
higher sensitivity to early developmental risk factors. These observations raise the possibility that expression of schizophrenia riskassociated genes may be different in placentae of male compared with female offspring, and this might relate to the greater incidence
of developmental disorders like schizophrenia among males ${ }^{2,5}$. We of developmental disorders like schizophrenia among males ${ }^{2,2}$. We
therefore tested whether PRS1 and PRS2 genes, which interact with ELCs on case-control status, are differentially expressed in placentae from male compared with female offspring. Analyses on pla-
cental samples from the two datasets with sex information revealed cental samples from the two datasets with sex information revealed
that PRS1 and PRS2 genes are highly significantly enriched among that PRS1 and PRS2 genes are highly significantly enriched among
the genes differentially expressed, and specifically upregulated, in the genes differentially expressed, and specifically upregulated, in $N=37,16$ females and 21 males, $P=2.3 \times 10^{-10} ;$ Fig. $\left.4 \mathrm{~b}\right)$. In the same datasets, the relative upregulation was also present in male preeclamptic placentae (GSE35574: $P=0.01$; GSE25906: $P=0.001$ ). Analogous analyses in a heart dataset (GSE4172) and a fetal lung dataset (GSE14334) with sex information did not reveal upregula-
tion of the PRS1 and PRS2 genes in males compared with females tion of the PRSI and PRS2 genes in males compared with females
$(P>0.40$; Supplementary information, see "Sensitivity analyses for placental enrichment" note). These data suggest a sex-biased role for the placenta in expressing genetic risk for schizophrenia.

Discussion

In this study, we show that exposure to ELCs represents an early environmental context that influences cumulative genomic risk for point, the set of genes within these genomic loci that show interaction with intra-uterine and perinatal complications is highly expressed in placenta, and the same set of genes displays differential enrichment in this organ in abnormal invasive placental states. These results suggest that the most significant genetic variants least partly by converging on a developmental traj shizophenta a intra-uterine and perinatal adversity and linked with abnormal placentation. Moreover, the strikingly relative enrichment of expression of schizophrenia risk genes in placentae from male compared with female offspring suggests that gene-environment interactions influencing placental biology may contribute to the higher incidence of schizophrenia in males compared with females schizophrenia, even as the syndrome is diagnosed during early and the developmental trajectory of schizophrenia. Schizophrenia is thought to be a condition on which the human species has a NATURE MEDICINE | VOL 24| JUNE 2018 | 792-801 | www.nature.com/naturemedicine

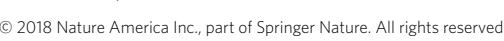

monopoly, and the delayed emergence of the clinical disorder evolved neural functions, such as prefrontal cortical cir highly Interestingly the evolutionary complexity of the primate placenta shows parallels with the phylogenetically remarkable expansion of the human brain, particularly prefrontal cortical regions that are among the most affected in schizophrenia ${ }^{53,54}$; both plaELCs in humans than in other species ${ }^{53,5}$. 5 . Our results are consistent with the possibility that some of the common genes implicated in late the physiology of the placenta, the risk of ELCs, and thereby secondarily the development of the brain, potentially interacting with other mechanisms of gene regulation acting primarily within fetal brain

pespris schizophrenia $5,1,1,1,5,57$, as well as autism ${ }^{5,59}$, the mechanisms by which this may happen have been elusive. Genetic research has been successfully focused on detecting GWAS significant variants, but the difficulty of collecting environmental data has hindered defining he developmental context in which these common variants may

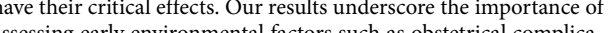
tions, in addition to genetic risk, to fully investigate their joimp effect on susceptibility to neurodevelopmental disorders. Our results atso point to the placenta as a crucial mediator of this interaction in relation to schizophrenia in particular, but likely to other neurodevelpenental disorders in general, underscoring the need for further esearch on placenta physiology in the context of brain developof prenatal care for reducing the burden of psychiatric illes role may identify new strategiese ${ }^{\circ 0}$ for placental health as a form of pimary prevention of schizophrenia, perhaps particularly in males Methods

Methods, including statements of data availability and any associated accession codes and refer

Received: 30 August 2017; Accepted: 16 March 2018

References

Saha, S., Chant, D. Wellham, J. \& McGrath, I. A systematic review of the
Shevalence of schizophrenia. PLoS Med. 2, el41 (2005).

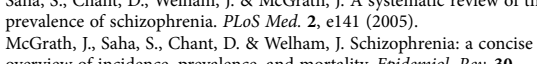

overview of incidence, prevalence, and mortality. Epidemiol. Rev. 30,
$67-76$ (2008). Sullivan, P. F. The genetics of schizophrenia. PLoS Med. 2, e212 (2005).
Lichtenstein, P. et al. Common genetic determinants of schizophrenia and bipolar disorder in Swedish families: a population-based study. Lancet 373,
$234-239(2009)$ Patterson, P. H. Ne Gottesman, I. I. \& Wolfgram, D. L. Schizophrenia Genesis: The Origins of Mikaelsson, M. A., Constancia, M., Dent, C. L., Wilkinson, L. S. \& Humby, T. Placental programming of anxiety in adulthood revealed by Igfi-null modes.
Nat. Commun. 4, 2311 (2013) Bronson, S. L. \& Bale, T. L. Prenatal stress-induced increases in placental

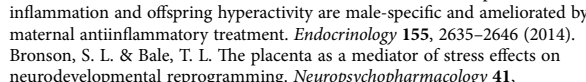
20urodevelopmental reprogramming. Neuropsychopharmacolology 41 ,
Bonnin, A. \& Levitt, P. Placental source for 5-HT that tunes fetal brain development. Neuropsychopharmacology $37,299-300$ (2012).
1. Weinberger D . . F rrom neuropathology to neurodevelopment Lancet 346 , Weinberger, D. 


\section{ARTICLES}

12. Brown, A. S. \& Derkits, E. J. Prenatal infection and schizophrenia: a review of 41. Wollmann, H. A. Intrauterine growth restriction: definition and etiology epidem.
(2010). 3. Cannon, M., Jones, P. B. \& Murray, R. M. Obstetric complications and
schizophrenia: historical and meta-analytic review. Am. I. Psychiatry 159

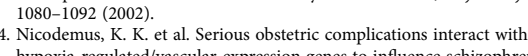
hypoxia-regulated/vascular-expression genes to influence schizophrenia risk. 15. Schmidt-Kastner, R., van O, I., Esquivel, G., Steinbusch, H. W. \& R Rutten, B. P. P. An environmental analysis of genes associated with schizophrenia: hypox
and vascular factors as interacting elements in the neurodevelopmental model. Mol. Psychiatry 17, 1194-1205 (2012).

Schizophrenia Working Group of the Psychiatric Genomics Consortium. Biological insights from 108 schiroph

17. Stefansson,, . et al. Common variants conferring risk of schizophrenia.
Nature 460, $744-777$ (2009). Ingason, A. et al. Copy number variations of chromosome 16p131.1 region

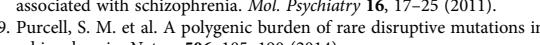

2. International Schizophrenia Consortium et al. Common polygenic variation contributes to ri:
$748-752(2009)$.

. Wray, N. R., Goddard, M. E. \& Visscher, P. M. Prediction of individual genetic risk to disease

McNeil, T. F. et al. Obstetric complications in histories of monozygotic tw 196-204 (1994).

23. McNeil, T. E., Cantor-Graae, E. \& Sjostrom, K. Obstetric complications as complication scales. J. Psychiatr. Res. 28, $519-530$ (1994).

Ursini, G. et al. Stress-related methylation of the catechol-O-methyl ransfers Val 158 allele predicts human prefrontal cognition and activity. J. Neurosci. Verdoux, H., Sutter, A. L., Glatigny-Dallay, E. \& Minisini, A. Obstetrical complications and the development of postpartum depressive symptoms:
a prospective survey of the MATQUID cohort. Acta Psychiatr. Scand. 106 212-219 (2002). 26. Wall, J. D. \& Pritchard, J. K. Haplotype blocks and linkage disequilibrium in
the human genome. Nat. Rev. Genet. 4, 587-597 (2003). the human genome. Nat. Rev. Genet. 4, $587-597$ (2003).

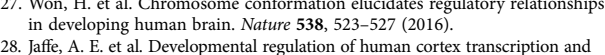
28. Jaffe, A. E. Et al. Developmental regulation of human cortex transcription and
its clinical relevance at single base resolution. Nat. Neuroscit 18, 154-161 (2015) its clinical relevance at single base resolution. Nat. Neuroscici 18, $154-11$
29. Jaffe, A. E. et al. Mapping DNA methylation across development, genotype and schizophrenia in the human frontal cortex. Nat. Neurosci. 19.

$40-47(2016)$.
Burton, G. I. $\&$ Jauniaux, E. The cytotrophoblastic shell and complications of
pregnancy Placenta $60.134-139(2017)$. pregnancy. Placenta 60, 134-139 (2017).
Cotechini, T. \& Graham, C. H G berrant pregnancy complications: . A. A. Aberrant maternal therapeutic inflammetet? Placion as a a caus 36 ,

32. Maltepe, E. \& Fisher, S. J. Placenta: the forgotten organ. Annu. Rev. Cell Dev. Maltepe epigenetic, and physiological integration during development. I. Clin. Invest
$120.1016-1025(2010)$ 120, 1016-1025 (2010).
Leavey, K. et al. Unsupervised placental gene expression profiling identifies 4. Leavey, K. et al. Unsupervised placental gene expression profiling identifi $137-147(2016)$

35. Redman, C. Pre-eclampsia: a complex and variable disease. Pregnane

36. Ferguson, K. K., Meeker, J. D., McElrath, T. F., Mukherije, B. \& Cantonwine D. E. Repeated measures of inflammation and oxidative stress biomarkers in
preeclamptic and normotensive pregnancies. Am. I. Obstet. Gynecol. 216, 527. Preeclamplitican nat).
e521-527.e529 (2017). 37. Redman, C. .. Sacks, G. P. \& Sargent, I. L. Preeclampsia: an excessive
maternal inflammatory response to pregnancy. Am. .. Obstet. Gynecol. 180, maternal inflammatory response to pregnancy. Am. J. Obste. Com
499-506 (1999). 8. Hwang, S. S. et al. Maternal substance use disorders and infant outcomes in
the first year of life among Massachusetts singletons, 2003-2010. I. Pediatr.

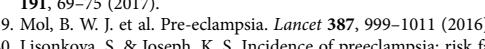
lisonkova, S. \& Joseph, K. S. Incidence of preeclampsia: risk factors and
outcomes associated with aerly- versus late-onset disease. Am. I. Obstet. 22. Dalman, C., Allebeck, P, Cullberg, J,., Grunewald, C. \& Koster, M. Obstetric
complications and the risk of schizophrenia: a longitudinal study of a national birth cohort. Arch. Ger. Psychiatry 56, 234-240 (1999).

43. Byrne, M., Agerbo, E., Bennedsen, B., Eaton, W. W. \& Mortensen, P. B.
Obstetric conditions and risk of first admiscion with schizophrenia:

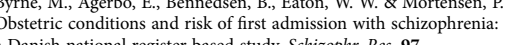

$51-59(2007)$.
44. Xiao, X. et al. HSF1 is required for extra-embryonic development, postnatal
growth and protection during inflammatory responses in mice. EMBO I. 18 , growth and protection during inflammatory responses in mice. EMBO. .18
$5943-5952$ (1999). 45. Hashimoto-Torii, $\mathrm{K}$. et al. Roles of heat shock factor 1 in neuronal response
to fetal environmental risks and its relevance to brain disorders. Neuron 82, $560-572$ (2014)

dematic review of the incidence of schizophrenia: the
d the influence of sex, urbanicity, migrant status and distribution of rates and the influence of sex, urbanicity, migrant status
dinethodology. BMC Med. 2, 13 (2004). methodology. BMC Med.
47. Aleman, A., Kahn, R. S. \& Selten, J. P. Sex differences in the risk of

schizophrenia: evidence from meta-analysis. Arch. Gen. Psychiatry 60 ,
5655771 (2003).

48. Hijorthoj, C., Sturup, A. E., McGrath, J. J. \& Nordentofit, M. Years of potenti
life lost and life expect meta-analysis. Lancet Psychiatry 4, 295-301 (2017).

9. Ochoa, S., Usall, J., Cobo, J., Labad, X. \& Kulkarni, J. Gender differences in schizophrenia and first-episode psychosis: a co
Schizophr. Res. Treatment 2012 , 916198 (2012)

0. Abel, K. M., Drake, R. \& Goldstein, J. M. Sex differences in schizophrenia

Int. Rev. Psychiatry 22, 417-428 (2010).

. Angermeyer, M. C., Kuhn, L. \& Goldstein, J. M. Gender and the course of
schizophrenia: differences in treated outcomes. Schizophr. Bull. 16, 293-307 (1990).
52. Bloom, B., Cohen, R. A. \& Freeman, G. Summary health statistics for U.S. children: National Health Interview Survey, 2010. Vital Health Stat. 10, 1-80 (2011).
53. Burton, G. J., Moffett, A. \& Keverne, B. Human evolution: brain, birthweight 53. Burton, G. I., Moffett, A. \& Keverne, B. Human evolution: brain, birh
and the immune system. Philos. Trans. R. Soc. Lond. B Biol. Sci. 370,
20140061 (2015).

54. Teffer, K. \& Semendeferi, K. Human prefrontal cortex: evolution,
development, and pathology. Prog. Brain Res. 195, 191-218 (2012).

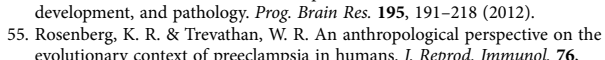
evolutionary context of preeclampsia in humans. I. Reprod. Immunol. 76,
$91-97$ (2007). 56. Sekar, A. et at al

A 2. Schizophrenia risk from complex variation of complement component 4. Nature 530, 177-183 (2016).

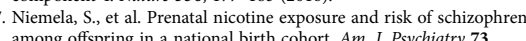
58. Glasson, E. J. et al. Perinatal factors and the development of autisn: a population study. Arch. Gen. Psychiatry $61,618-627$ (2004).
59. Stoner, R. et al Patches of disorganiaty autism. N. Engl. I. Med. 370, 1209-1219 (2014).

60. Ross, R. G. et al. Perinatal phosphatidylcholine supplementation and early
childhood behavior problems: evidence for CHRNA7 moderation. Am. I.
Psychiatry 173, 509-516 (2016).

\section{Acknowledgements}

Ack are grateful to the Lieber and Maltz families for their visionary support that
funded the analytic work of this project. We thank all of the participants in the We are gratefut to the tieber and Malt families for their visionary support that
funded the analytic work of this project. We thank kall of the participants in the
study and their families. We thank loo Heon Shin for help with the gene expression study and their families. We thank Joo Heon Shin for help with the gene expression
analyses, and Barbara Gelao, Marina Mancini, Raffaella Romano, Rita Maseslis, and
Grazia Caforio for help with data acuisition. We also thank Sally Cheung and John Meyer for data management, and Susan Fisher for guidance with placental datasets providing the statistics for PRS calculation, and Thomas F. McNeil for providing the scale for ELC scoring. We also thank all of the authors of the publicly available placental dite the Intramural Research Program of the NIMH to the Clinical Brain Disorders

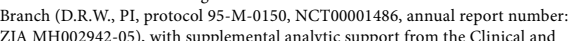
Translational Neuroscience Branch (K. F.B., P.). G.U. received partial support from
P50MH 094268 . The collection of ELCs and genetic data for the Italian sample has been supported by the NARSAD grant no. 20337 and the "Ricerca Finalizzata" grant supported by funding from the Max Planck Society, the Max Planck Förderstiftung,
the DFG ( CNMPB) EXTRABRANEUE-FP, and the Nivedersachsen-Research
Network on Neuroinfectiology (N-RENNT) (to H.E.).

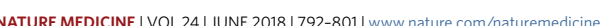

Author contributions

E.G.G.P., and D.R.W. designed the study and interpreted the results. G.U., G.P., J.F.R and D.R.W. organized and performed genotyping, imputation, and risk profile scoring the discovery sample and in G.M., M.B., H.Y. R.H., D.R., and H.E. carried out ELC assessment. .F... and E.G.H. and, together with G.U., G.P. C.C., and D.R.W., interpreteded the resullts of the gene set enrichment analyses in placental samples from complicated pregnancies compared with
controls. G.U. G.P., and D.R. .. . drafted the manuscript, and all authors contributed to controls. G.V., G.P., and D.Rew.
the final version of the paper
Competing interests

Additional information S41591-018-0021

(1) Publisher's note: Springer Nature remains neutral with regard to jurisdictional claims in published maps and institututional affiliations 
Methods

Compliance with ethical requirements All procedures performed in the clinical samples were in accordance with the ethical standards of the institutional research
committees and with the 1964 Declaration of Helsinki and its later amendments or comparable ethical standards. Informed consent was obtained from all individual
participants included in this sudy

\section{Samples and genotyping}

Discovery sample (USA): scz_lieeur. A total of 501 Caucasian unrelated adult
subjects, with good-quality genetics data and ELC history information (as Branch Sibling Strected from participants in the Clinical Brain Disor-
B ders Branch Sibling Study of Schizophrenia at the National Institute of Men-
tal Health (NIMH; Clinical Brain Disorders Branch, protocol 95-M-O150 NCT00001486, Annual Report number: ZIA MH002942-05). The sample
included 234 patients who met Digagnstic and Statistical Manual of Mental
Disorders, Fourth Edition (DSM-IV) criteria for schizophrenia and 267 healthy subjects (see Supplementary Table 2 for details). The Institutional Review Boar
of the NIMH Intramural Program approved the study und witten inform consent was obtained from the participants affer complete description of the
study. Exclusion and inclusion criteria have exen previously yeported

First replication sample (Italy): scz-bari_eur. A total number of 273 Italian
Caucasian unrelated dadult subjects ophrenia cases and 182 controlss see Supplementary Table 2 for details) wi
availability of genetics data and ELC history inforyt tab similer

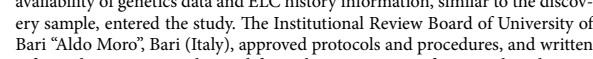
Bari "Aldo Moro," Bari (Italy), approved protocols and procedures, and written
informed consent was obtained from the participants after complete descrip
tion of the study. Fxclusion and inclusion criteria were similar to the discovery sample, as reported elsewhere $e^{2}$. Genotyping was done using Illumina Bead

Second replication sample (Germany): scz_munc_eur. A total of 919 Caucasian
unrelatated adult subjects entered the studd (551 schizophrenia cases and 298 controls; see Supplementary Table 2 for details). Cases were ascertained from
the Munich area of Germany, as described previously" $y^{\prime \prime}$. The controls were unrelated volunteers randomly selected from the general population of Munich.
All were screened to exclude a history of psychosis central neurological dis-

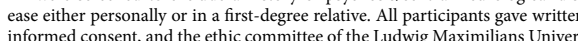
informed consent, and the ethic committee of the Ludwig Maximilians Univer
sity, Munich (Germany), aproveded the human subjects protocols. Genotypin was done with the Illumina $317 \mathrm{~K}$ arrey

Third replication sample (Germany): scz_raseeur. The GRAS collection
included 1,020 unrelated adult patients with schizophrenia (see Supplementary Table 2 for details), recruited across 23 German hospitals. Cases com-
pleted a structured clinical interview and were diagnosed with DSM-IV pleted a structured
schizophrenia or schizoaffective disorder ${ }^{3}$ s.t. Georg-August-University ethics committee and Ethics Committee of the
University of Göttingen, Göttingen (Germany). All participants gave written enotyping array (Affymetrix, Santa Clara, CA, USA), based o casian residents of Europen

Fourth replication sample (Eastern Asia): scz_osak_asi. A total of 172 Japa-
nese unrelated adult patients who met DSM-IV criteria for schizophrenia (see Supplementary Table 2 for details) with availability of genetics data an
ELC exposure information, similar to the discovery sample, entered the stud ELC exposure information, similar to the discovery sample, entered the stud
The Institutional Review Board of University of Osaka, Osaka (Japan), approved

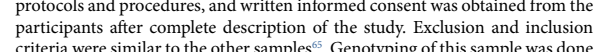
using the Affymetrix Genome-Wide Human SNP Array 6.0 (Affymetrix, Santa
Clara, CA, USA). Quality control for genotyping. Quality control was performed using PLINK
(version 1.07; http:///pngu.mghh.harvard.edu/purcell/plink//), consistent with previous reference, for the scz_lie_eur, scz_bari_eur, scz_munc_eur, and
scz_osak asi samplesis scz_osak_asis sampleses . Participants with missing rate higher than $2 \%$ and extren
heterozygosity values ( \pm 3 s.d.). were removed. SNPs with missing rate higher than $2 \%$ and iffference in missingness between cases and controls $>0.02$ were
also removed. II addition, SNPs were excluded if they failed Hardy-Weinberg equilibrium $\left(P<10^{-6}\right.$ in controls or $P<10^{-10}$ in cases) and if they had minor allele frequency less than $1 \%$. Prephasing was done before imputation using SHAPEIT,
and imputation was done using IMPUTE2 with Phase I 1000 genome as the

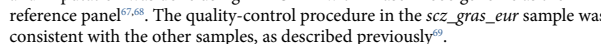

Derivation of polygenic risk profile scores. Cumulative genetic risk profile scores
(PRSs) ${ }^{21}$ were calculated for each individual as described ensewheres (PRSS)
PRSs are a meare calculated for each individual, as described elsewherere for schizophrenia from the recent GWAS studd'620. We thus multiplied the natural
log of the OR of each index SNP. from this recent schizophrenia GWAS', by the mputation probability for the corresponding referennce allele at each variant, and summed the products over all ariants, so that each subject had whole genome
PRSs as originally described for this measure $e^{2021}$. The PGC provided ORs and NPs were derived from a meta-analysis of PGC GWAS datasets excluding o

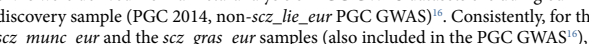

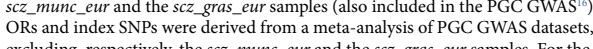
excluding, respectively, the scz_munc_eur and the scz-gras eur samples. For the Te PGC GWAS datasets, because these samples are not included in the PGC only autosomal SNPs were included in the analysis, to prevent any bias related to
ons exin the PRS calculation. We performed a linkage disequilibrium (LD) pruning with, another f $($ the SNPs, discarding variants within $500 \mathrm{~kb}$ of, and in $r \geq 0.1$ (PRS1-10) were calculateded using subsets of SNPS selected according to the GWAS

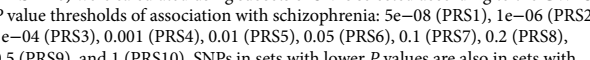
(A) $P$ and ( higher P Palues (for example, SNPS in PRS1 are included in PRS2, SNPS in PRS2
are included in PRS3, and so on). A detailed list of SNPS included in PRS1 and
PRSS is is provided in Supplementary Thable 1 . We performed all of the analyses bot PRS2 is provided in Supplementary Table 1. We performed all of the analyses bo including and excluding the top GWAS significant SNP in the extended MHC
locus (hy19 coordinates: chrr: 25-34 Mb), with similar results (Supplementary Table 4). For additional analyses (Supplementary Fig. 21; Supplementary

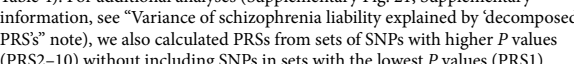
Assessment of early-life complications. ELCs are here referred to as "somatic neonatal period" potentially harmurul for the offspring, with special focus on the to

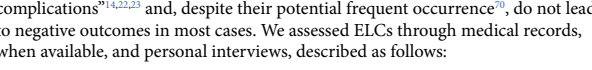
$s c z$ lie_eur, and $s c z$ barizeurr. We used mainly maternal recall based on an
extensive personal interview, which has been repeatedly shown to represents extensive personal interview, which has been repeatedly shown to represent a
reliable method for obtaining ELC history, when used in a careful and detailed

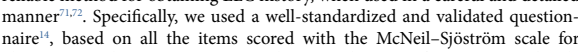
obstetric complications $5^{223}$. It covers the entire duration of pregnancy and early
neonatal life, and also contains indicators of reliability and an assessment of the . Wh scz_osak ass: We used mainly medical records. When medical records were not
exhaustive, we interviewed the mothers of the patients; the histories were again

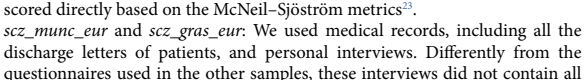
discharge letters of patients, and personal interviews. Differently
questionnaires used in the other samples, thes interviews did not contain all
the items included in the McNeil-Sijostrom scales the items included in the McNeil-Siöstrom scale ${ }^{33}$, thus increasing the risk for
incomplete information. History of ELCS from the available information was

In the McNeil-Sjostrtrom scale ${ }^{22323}$ each ELC is assigned a severity level of $1-6$,
based on the degree of inferred potential harm to the offspring central nervous system. ELCS with severity weight $\geq 4$ are considered potentially clearly harmful
or relevant factors in fetal stress The McNeil-Sijstrom scale in the cor of maternal recollection has been well validated in comparison with hospital

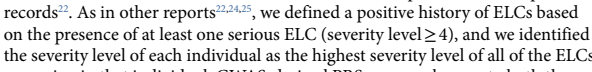
occurring in that individual. GWAS-derived PRSS were unknown to both the
individuals who provided the information about ELCs and to the researchers provided was incomplete or inconsistent (for example, contradictory answers to
related questions), or if the presence of a complication was certain but a severity weight could not be confidently determined. The frequency of ELCS in our samples may be not representativive of the general populations (Supplementary information, see "Considerations about the assessment and the requenency of early
life complications (ELCS)" note). Supplementary Table 21 contains a list of the life complications (ELC)" no
ELCs detected in each sample.

Statistical analysis of the interaction between polygenic risk scores

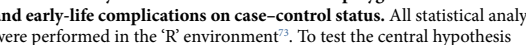

were performed in the '
of 'environmentist. 'To test the central hypothesis

MATURE MEDICIE/

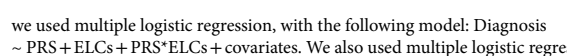
to confirm the association of each PRS with case-control status in our samples (Diagnosis $\sim$ PRS + covariates) and to verify whether ELCS were associated with
schizophrenia (Diagnosis $~$ ELCSs+ covariates). In the presence of an interaction

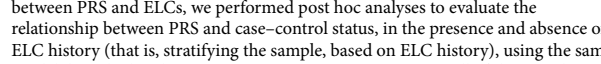
ELC history (that is, stratifying the sample, based on ELC history), using the same
model described earlier (Diagnosis $\sim$ PRS + covariates). For all of these analyses, we report in the main text the P $P$ alues and the $t$-statisitics associated with our
variable of interest (that is, PRSEELCs, or PRS). To evaluate goodness of fit of these variable of interest (that is, PRS*ELCS, or PRS). To evaluate goodness of fit of these
logistic models (Diagnosis $\sim$ PRS + covariates) in the whole sample, in the absence logistic models (Diagnosis $\sim$ PRS + covariates) in the whole sample, in the absence
and presence of ELC history, we calculated the Nagelkerke $R^{2}$, by comparison of a
full model (covariates +PRS) with a reduced model (covariates only) Similarly, in the presence of an interaction between PRS and ELCS on case-control status, we performed post hoc analyses to test the relation between PRS and ELCS, separately
in controls and in patients with schizophrenia (that is, after stratifiying the sample for diagnosis), using multiple logistic regeression (ELC history $\sim$ PRS + covariates.
Consistently with the interaction between PRS and ELCS, we found a positive relation between PRS and ELCS only in patients with schizophreniaz we fiut
explored this relationship in the two replication samples of only patients

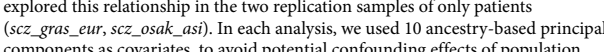
components as covariates, to avoid potential confounding effectsto of population
stratificationion, consisistent with previous work ${ }^{6}$. We performed sensitivity analyses adding sex, age, maternal and paternal ages, maternal stress, history of substance

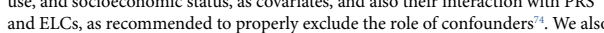
performed sensitivity analyses, in each sample, by excluding the individuals with mothers with a history of substance use. Results were consistent and are reported
in Supplementary Tables $6-8$. For the analyses in the merged sample, PRS scores were normalized by subtracting the mean and dividing for the s.d. in each sample for this analysis, we added the sample as a covariate.

Selection of PRS1 and PRS2 genes. To define genes mapping to the PRS1 and
PRS2 loci for gene set analyses, we used two alternative criteria:

PGC LD regions: We considered, as PRS1 and PRS2 genes, all of the University
of Californian Santa Cruz (UCSC) genes overlapping the LD regions associtated
with each SNP $\left(R^{2}>0.6\right.$. as reported in a previous referencel with each SNP $\left(R^{2}>0.6\right)$, as reported in a previous referencele ${ }^{16}$ and on the $P G C$
website (http: Distance: We considered, as PRS1 and PRS2 genes, all of the UCSC genes map-
ping $500 \mathrm{obb} \pm$ the index SNPPs of each PRS in the discovery sample (scz_lie__urr). that LD differs among populations, as we analyzed multiple samples. Moreovers the LD regions associated with each SNP have a huge variability: for example

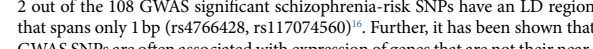
est genes and are outside the associated LD regions 6 6 65 . Finally, the distance of

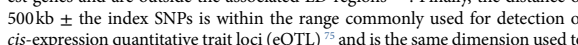

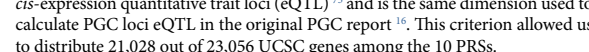
Differences between the two list of genes (reported in Supplementary Table $9 a, b)$ are related not only to the criterion adopted for SNP selection (distance or LD), bu
also to the fact that the PGC loci associated with schizophrenia at $P<5 \times 10^{-8}$ deCODE data, whereas SNPS for PRS calculation are derived from the primary
GWA only'.6 Because only SNPs mapping to autosomal chromosomes are used for schizophrenia PRS construction tas, we excluded from our analysis genes
that were irrelevant to our question, that is, genes mapping to mitochondrial DNA wan X-and Y-chromosomengenens or other menens mapping to locin not used
Dor PRS calculation. After exclusion of the genes on sex chromosomes and on for PRS calculation. After exclusion of the genes on sex chromosomes and on
mitcochondrial DNA and genes undetected in the expression datasests analyzed,

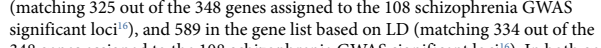
348 genes assigned to the 108 schizophrenia GWAS significant loci'i"). In both gen
lists, PRS1 genes are a subset of PRS2 genes (thereforer ereferred in the text as PRS1 and PRS2 genes). We performed all of the gene set analyses, with PRS1 and PRS genes defined with both criteria (LD and distance), and found consistent results
(Supplementary Table 9 ). In the main text, we report results with the PRS1 and
PRS2 genes defined based on the distance criterion (Table 2).

Expression of PRS1 and PRS2 genes in placenta. The proprietary placental tissue
used for this analysis was collected at the University of California, San Francisco. Methods of collection were approved by the University of California, San Francisco
Institutional Review Board, and informed consent was obtained from all donors. Institutional Review Board, and informed consent was obtained from all donors
The RNA-sequencing datasets related to second trimester and term placental
tisssues (amnion, basal plate, chorion, villi) and isolated cells trophoblasts are NATURE MEDICINE | www.nature.com/naturemedicine publicly available (GSE16368) on the National Institutes of Health Roadmap
Epigenomics Project website (http://www.roadmapepigenonomics.orgy). Expression

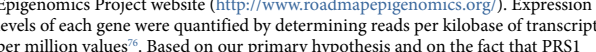
per million valueses".
and Pased on our primary hypothesis and on the fact that PRSS
and PRS risk SNPS are associated with expression of nearby genes across many different tissues (Supplementary information, see "Screening of PRS1 and PRS2 to the loci showing the strongest association with schizophrenia and interacting
with ELCS are more expressed in placenta compared with randomly selected genes

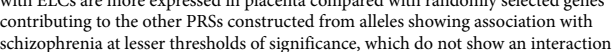
with ELCs. To perform this analysis, we used the function genesetTest' in the

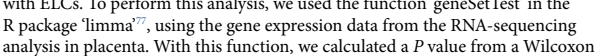
analysis in placenta. With this function, we calculated a $P$ value from a Wilcoxon
est to verify the hypothesis that the selected set of genes (PRS1 and PRS2) ends to be more highth ranked in expression compared with randomly selected
sets of genes of the same size (from the PSS3 10 genes) . $e$ sults are reported in Supplementary Table 10 . We also performed a further analysis testing the
enrichment of the genes overlapping the PRS1 and PRS2 loci using the function

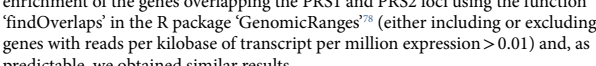

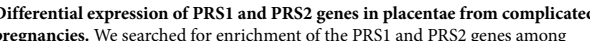
pregnancies. We searched for enrichment of the PRS1 and PRS2 genes among
genes differentially expressed in placental samples from complicated pregnancies Expression Omnibus public repository. Datasets were chosen for analysis if they included all of the following: a comparison between placental samples from complicated pregnancies and controls, more than one sample per group (that is,
comparisons between pooled RNA samples were discarded), and expression datt for at least half of the PRS genes. We found eight datasets that met these criteria,
comparing control versus diseased (pre-eclampsia and IUGR) placenta cells. comparing control versus diseased (pre-eclampsia and IUGR) placenta cells.
A dataset on normal cultured cytotrophoblasts was also chosen as cells were

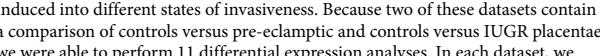
we were able to perform 11 differential expression analyses. In each dataset, we
dropped probes that map to multiple genes and, when more than one probe per gene was present, we selected the one with hhe highest mean expression. We used
the function eBayes in the $R$ package limma't to attribute a moderated $t$-statistic to each gene related to differential expression (using the covariates provided by -statistics (results are reported in Table 2) testing whether the selected set of genes (that is those related to PRS1 and PRS2) tends to be more highly ranked in
differential expression compared with randomly selected genes of the same size from the other GWAS loci (PRS3-10). We also used a $\chi^{2}$ test to confirm whether
PRS1 and PRS2 genes were enriched for differentially expressed genes compared with the remaining genes (Table 2).

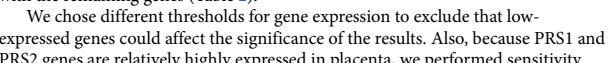
nalysisens arjusting for for average gene experessin placenta, we performed sensitivity nnalysis, adjusting for average gene expression: for this purpose, we assigned eac
gene a moderated t-statistic from the differential expression analyses, an in-set" value " "to PRSI and PRS2 genes, and an "insert" value " 0 " to the remaining and the 'in-see' variable, affer covarying for average gene expression (see results in Supplementary Table 11). Importantly, in addition to an enrichment analysis
of PSS1 and PRS2 2 genes based on genes mapping 500 obb \pm the index SNPPs of genes, defined as the UCSC genes overlapping the LD regions associated with each SNP $\left(R^{2}>0.6\right)$, as reported on the PGC website (http:///www.med. unc.ed and PRS2 genes are entriched among the genes that are differentially expressed in placentae from male compared with female offspring. Among the placental
datasets selected in our study, only three " "GSE25861", "GSE35574", "GSE25006") contained sex information, one of them ("GSE25861") included on e emale sample
Therefore, we limited this anl "GSE25906" (Fig. 4). Also, in this case, we performed sensitivity analyses adjusting for average gene expression (Supplementary Table 1
To confirm the specificity of our findings in the

pregnancies, we performed simllar analyses in available datasests from normal affected organs and in embryonic cells under distress (results for all of these sensitivity analyses are reported in Table 2 , Supplementary Tables 9 and 11 , and
Supplementary information, see "Sensitivity analyses for placental enrichment" note.

Placental-enriched risk profile scoring. We calculated PRSs based on the GWAS
SNSs markcing loci-contanining genes hibhly expressed in placenta and differentially SNPs marking loci-containing genes highly expressed in placenta and differenti
expressed in placentae from complicated pregnancies, and compared their
interaction with ELCS to PRSs derived from the SNPs marking the remaining 


\section{ARTICLES}

GWAS significant loci. For this purpose, we selected the loci-containing genes $\quad$ repository (https:///www.ncbi.nlm.nih.gov/geoo/) under the accession codes the locicicontaining genes with expression in the oupper decile both in trophoblast and in vilit. This gave us a IIS Oo S6 SNPs for PRSI and 112 SNPs for PRS2, as calculated PRSs based on these SNPs “"Placentu"l" risk profile scores PlacepPS and PlacPRS2) and on the remaining SNPS in these PRS1 and PRSS loci that
did not show high or differential expression in placental tissues (NonPlacPRS risk profile scores" section). In an analogous way and to address the organ specificity of he lacrRS interaction whin $\mathrm{LLC}$, we calculated "TissuePRSs" and highly and differentially expressed in addltt and fetal tisswes or ar associnted with methylation quantitiative trait loci in postmortem human dorsolateral preffron cortex, or with che Tisup PSS and NonTissuePRSs with ESCs andyed the status. Results of these sensitivity analyses are reported in Supplementary Tables
$12-14$ and in the Supplementary information (see "Sensitivity analyses for placental enrichment" note).

Pathway and functional analyses. We investigated whether the placenta-enriched
genes mapping to the loci of PlacPRS1 and PlacPRS 2 are enriched for particular genes mappling to he bo and PRS2 loci (NonllacrRSS and Non laclacPSS2). Data were analyzed through
and QIAGENs Ingenuity Pathway Analysis (QIAGEN, Redwood City, CA, USA;

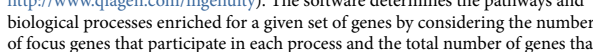
are known to be associated with that process in the selected reference set. We
and (reference set: Ingenuity Knowledge Base, relationships: direct and indirect; node types: all; data sources: all; confidence: experimentally observed and high; spectese $P$ value calculation based on the Beniminin-Hochberg metho multiple testing in the canonical pathway and functional analyses. In addition, we used the Pan PlacPRS2 genes and NonPlacPRS1 and NonPlacPRS2 genes show differences in biological processes, and celluluag componentst. In this analysis, the P Pvalue calculatat Reporting Summary. Further information on experimental design is available

1. Rasetti, R. et al. Altered cortical network dynamics: a potential intermediate 6henotype for schizophrenia and
Psychiatry 68, 1207-1217 (2011).

政

schizophrenia. Epigenetics $11,1-13(2016)$.
63. Begemann, M. et al. Modification of cognitive performance in schizophrenia complexin 2 gene polymorphisms. Arch. Geen. Psychiatry 67, 879-888
64. Ribbe, K. et al. The cross-sectional GRAS samplea a conprehensive phenotypical data collection of schizophrenic patients. BMC Psychiatry 10 65. Ohi K Ket tworks implicate cognitive impairments in

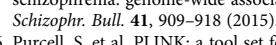

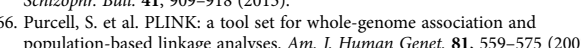

population-based linkage analyses. Am.J. Human Genet. $81,559-575$ (2007).
67. Howie, B., Marchini, J. \& Stephens, M. Genotype imputation with thousands

of genomes. G3 (Bethesda) 1, 457-470 (2011).

for thousands of genomines I.\& Zagury, J. F. A linear complexity phasing method

6. Stepniak, B. et al. Accumulated environmental risk determining age at $444-453$ (2014) Danllack, V. A., Nunes, A. P. \& Phipps, M. G. Unexpected complications of
low-risk pregnancies in the United States. Am. I. Obstet. Gynecol. 212, 809 .

el-809.e6 (2015).
71. Hewson, D. \& Bennett, A. Childbirth research data: medical records or
women's reports? Am. J. Epidemiol. 125, 484-491 (1987).

72. O'Callaghan, E, Larkin, C. \& Waddington, J. L. Obstetric complications in
schizophrenia and the validity of maternal recall. Psychol. Med. 20, 89-94 (1990)

73. R Development Core Team. R: A L Lannuagege and Environment for Statisitical

Computing (R Foundation for Statistical Computing, 2008).
74. Keller, M. C. Gene x environment interaction studies have

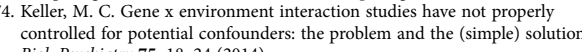
Biol. Psychiatry 75, 18-24 (2014). 75. Zhu, Z. et al. Integration of summary data from GWAS and eQTL studies 76. Roadmap Epigenomics Consortium et al al. Integrative analysis of 111 reference

77. Ritchie, M. E. et al. limma powers differential expression analyses for
RNA-sequencing and microarray studies. Nucleic Acids Res. 43 , e47 (2015).

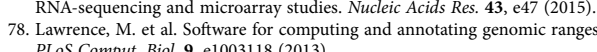
79. Mi, H., Muruganuian A Casegrande, I. T. \& Thomas, P. D. I function analysis with the PANTHER classification system. Nat. Protoc. 8,
$1551-1566$ (2013).

netic and ELC data generated and analyzed during this study are available from the corresponding athor on reas used for the analyses. The placental datasets and the other gene expression
datassets analyzed in this study are available on the Gene Expression Omnibus 



\section{ARTICLE}

\section{Uncoupling the widespread occurrence of anti-NMDAR1 autoantibodies from neuropsychiatric disease in a novel autoimmune model}

Hong Pan ${ }^{1} \cdot$ Bárbara Oliveira $^{1} \cdot$ Gesine Saher $^{2} \cdot$ Ekrem Dere $^{1} \cdot$ Daniel Tapken $^{3} \cdot$ Marina Mitjans $^{1} \cdot$ Jan Seidel $^{1}$. Janina Wesolowski ${ }^{1}$ Debia Wakhloo ${ }^{1}$. Christina Klein-Schmidt ${ }^{3}$ - Anja Ronnenberg ${ }^{1}$ Kerstin Schwabe ${ }^{4}$. Ralf Trippe ${ }^{3} \cdot$ Kerstin Mätz-Rensing $^{5} \cdot$ Stefan Berghoff ${ }^{2} \cdot$ Yazeed Al-Krinawe $^{4} \cdot$ Henrik Martens $^{6} \cdot$ Martin Begemann $^{1}$ Winfried Stöcker ${ }^{7} \cdot$ Franz-Josef Kaup $^{5} \cdot$ Reinhard Mischke $^{8} \cdot$ Susann Boretius $\oplus^{9} \cdot$ Klaus-Armin Nave ${ }^{2,10}$ Joachim K. Krauss ${ }^{4} \cdot$ Michael Hollmann $\oplus^{3} \cdot$ Fred Lühder $^{11} \cdot$ Hannelore Ehrenreich ${ }^{1,10}$ Received: 29 August 2017 / Revised: 20 October 2017 / Accepted: 30 October 2017 / Published online: 9 February 2018
O The Author(s) 2018. This article is published with open access

Abstract

Autoantibodies of the IgG class against N-methyl-D-aspartate-receptor subunit-NR1 (NMDAR1-AB) were considered pathognomonic for anti-NMDAR encephalitis. This view has been challenged by the age-dependent seroprevalence (up to $>20 \%$ ) of functional NMDAR1-AB of all immunoglobulin classes found in $>5000$ individuals, healthy or affected by different diseases. These findings question a merely encephalitogenic role of NMDAR1-AB. Here, we show that NMDAR1$\mathrm{AB}$ belong to the normal autoimmune repertoire of dogs, cats, rats, mice, baboons, and rhesus macaques, and are functional in the NMDAR1 internalization assay based on human IPSC-derived cortical neurons. The age dependence of seroprevalence is lost in nonhuman primates in captivity and in human migrants, raising the intriguing possibility that seroprevalence is lost in nonhuman primates in captivity and in human migrants, raising the intriguing possibility that chronic life stress may be related to NMDARI-AB formation, predominantly of the IgA class. Active immunization of $A P O E$ and $A P O E$ mice against four peptides of the extacellular NADARI domain or ovalbumin (control) leads to high circulating levels of specific AB. After 4 weeks, the endogenously formed NMDAR1-AB ( $\mathrm{gGG}$ induce psychosis-like symptoms upon MK-801 challenge in $A p o E$ mice, characterized by an open blood-brain barrier, but not in their $A p o E$ littermates, which are indistinguishable from ovalbumin controls. Impotantly, NMDARI-AB do not induce any sign of inflammation in the brain. Immunohistochemical staining for microglial activation markers and $\mathrm{T}$ lymphocytes in the hippocampus yields comparable results in $A P O E$ and $A p o E$ mice, irrespective of immunization against NMDAR1 or ovalbumin. These data suggest that NMDAR1-AB of the IgG class shape behavioral phenotypes upon access to the brain bu do not cause brain inflammation on their own

\section{Introduction}

Autoantibodies $(\mathrm{AB})$ of the immunoglobulin $\mathrm{G}(\mathrm{IgG})$ class against the N-methyl-D-aspartate-receptor subunit-NR1 (NMDAR1) were originally interpreted as pathognomonic for a condition called "anti-NMDAR encephalitis", characterized by high serum and cerebrospinal fluid (CSF) titers

Hong Pan, Bárbara Oliveira, and Gesine Saher contributed equally to

$\triangle \begin{aligned} & \text { Hannelore Ehrenreich } \\ & \text { ehrenreich@em.mpg.de }\end{aligned}$

of these $\mathrm{AB}$, as well as a variably favorable response to immunosuppressive therapy. The reported syndrome, reflecting typical NMDAR1 antagonistic actions, consisted of psychosis, epileptic seizures, dyskinesia, cognitive decline, reduced consciousness, and autonomic dysregulation [1-4]. However, work on $>5000$ individuals, healthy or affected by different diseases, consistently revealed overall comparable ge-dependent seroprevalence of functional NMDAR1-AB of all Ig classes, nurturing serious doubts regarding a purely pathological role of NMDAR1-AB of any Ig class [5-10]. NMDAR1-AB apparently belong to a pre-existing autoimmune repertoire [11-17], where Ig isotypes are determined by extracellular vs. intracellular antigen location [6]. This may explain the rarity of the IgG class among $\mathrm{AB}$ directed against extracellular epitopes, e.g., NMDAR1,
MOG, and CASPR2. In contrast, $A B$ that recognize intracellular antigens, e.g., amphiphysin, ARHGAP26, or GAD65, show predominance of IgG [6]. Despite this apparent "physiological autoimmunity", no report exists that systematically screened mammals other than humans for the presence of NMDAR1-AB. In recent work, we found that all naturally occurring NMDAR1-AB are functional and thus have pathogenic potential irrespective of epitope and Is class [10]. Pathophysiological significance may emerge in conditions of compromised blood-brain brier (BBB), fo instance, upon injury, infection, inflammation, or genetic predisposition (APOE4 haplotype), which then allows predisposition (AP OE where they act as NMDAR ants $[5,9,18-20]$. where Alternaty brain and produce late a in the 21]. The $[14,21]$. The question whe abundant endogenously produced . "

The present paper has therefore been designed to (i) sysThe present paper has therefore been designed to (i) systematically screen mammals other than humans for seroprevalence of functional NMDARI-AB and (ii) study mice wh open BBB behawal and morphological consequences the IgG class formed in response to immunization.

\section{Materials and methods}

\section{Ethical approvals}

Ethics committees of Georg-August University, Göttingen, and collaborating centers approved the Göttingen Research Association for Schizophrenia (GRAS) data collection and other studies "extended GRAS" acquiring human data, serum samples, and IPSC $[5,6,8,9,22,23]$. Hannover Medical School Ethics Committee approved the neurosurgical specimen collection. Studies comply with Helsinki Declaration. Patients gave written informed consent. Mouse studies were approved by Animal Ethics (LAVES, Oldenburg) following German Animal Protection Law.

Notes: All experiments were performed by researcher unaware of group assignment. The new nomenclature GluN1 for NMDAR1 is mostly disregarded here for consistency with the respective literature.

\section{Human samples}

GRAS and "extended GRAS"

The GRAS [22, 23] subsample used here consists of deepphenotyped patients $(N=970$; age $39.29 \pm 0.40$ years; $66.3 \%$ men), diagnosed with schizophrenia or schizoaffective disorder according to DSM-IV-TR [24]. Subjects of "extended GRAS $(N=4933$; age $43.29 \pm 0.24$ years; $56.9 \%$ men) comprise healthy individuals and patients with different neuropsychiatric diagnoses, including schizophrenia, affective disorders, multiple sclerosis, Parkinson, ALS, stroke, and personality disorders (detailed description in $[5,6,8,9]$ ). For this study, subjects are dichotomously classified as nonmigrants or migrants comprising first (patient migrated) and second generation (parents migrated). Identified migrants $(N=301 / N=4933)$ are from Europe $(49.8 \%)$, Asia $(36.9 \%)$, Africa $(9 \%)$, North America $2 \%)$, South America $(0.7 \%)$, or mixed $(1.6 \%)$

\section{Neurosurgical patients}

A total of $N=72$ paired samples of serum and ventricular CSF were available from patients $(N=45$ women; age $55.9 \pm 2.2$ years; $N=27$ men; age $60.2 \pm 2.7$ years) undergoing neurosurgery for various reasons: meningiomas, metastases, and other brain tumors $(N=25)$; intracerebral/subarachnoid hemorrhages $(N=20)$; hydrocephalus $(N=12)$; arterial aneurysms $(N=7)$; trigeminal neuralgia $(N=4)$; and other $(N=4)$ Most pairs were taken simultaneously at the time poin of surgery, i.e., $<5 \min (N=64)$ or $<30 \min (N=8)$ apart.

\section{Other mammals}

\section{Dogs and cats}

Serum samples from dogs and cats of different breeds were prospectively collected during routine (health check/vaccination) or diagnostic (spectrum of different disorders) workup of outpatients in the Small Animal Clinic, University of Veterinary Medicine, Hannover

\section{Monkeys}

Serum samples from healthy baboons and rhesus macaques were obtained through routine checkups at the Leibniz Institute for Primate Research, Göttingen.

Rodents

Serum samples from healthy rats and mice were obtained at the Max Planck Institute of Experimental Medicine and the Institute for Multiple Sclerosis Research, Göttingen.

\section{Serological analyses}

NMDAR1-AB determination by clinical standard procedures

Human serum and ventricular CSF were tested fo NMDAR1-AB positivity using commercially available kits, 
based on HEK293T cells transfected with NMDAR1 and secondary $\mathrm{AB}$ against human IgG, IgM, or IgA (Euroimmun, Lübeck, Germany) [2, 25]. Mouse serum was analyzed using the same assay with secondary AB against
mouse IgG, IgM, or IgA (M31001, A-31570, A-21042 Thermo Fisher, Rockford, USA).

\section{NMDAR1-AB IgM screening in monkey samples}

HEK293T cells $(50,000)$ cultured at $37^{\circ} \mathrm{C} / 8 \% \quad \mathrm{CO}_{2}$ in DMEM (high glucose, Life Technologies, Carlsbad, USA) were seeded on a $35-\mathrm{mm}$ dish, grown for 3 days, and transfected with $3 \mu \mathrm{g}$ of myc-His-tagged GluN1-1b cloned into pcDNA4/TO/myc-His A (Invitrogen, Carlsbad, USA) using Metafectene-Pro (Biontex, Munich, Germany) [10] One day post transfection, cells were split onto five poly-D lysine-coated coverslips in a 35-nim dish and 1 day later, they were fxed wh $5 \%$ parafority min, washed $5 \times$ (PBS), permeabilized with $0.1 \%$ Triton $X$ $5 \%$ for 5 min, again washed $5 \times$ (PBS), and blocked with $5 \%$ normal goat serum (NGS, Sigma-Aldrich, Munich, Germany) for $1 \mathrm{~h}$. After five PBS washes, cells were incubated with serum and monoclonal mouse anti-myc $\mathrm{IgC}$ (clone 9E10, Hollmann-Lab, Bochum) for $1 \mathrm{~h}$, washed

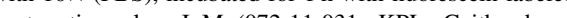
goat anti-monkey IgM (072-11-031; KPL, Gaithersburg, SA) and Alexafluor@594-labeled goat anti-mouse IgG (A11005; Thermo Fisher) secondary AB, and PBS washed $5 \times$. Cells were mounted in Fluoromount- $C$ (Southern Biotech, Birmingham, USA) and analyzed via TCS-SP2-AOBS confocal microscope $(63 \times$ oil immersion objective; Leica-Microsystems, Wetzlar, Germany). The results were independently assessed by three investigators.

\section{Protein-A assay}

Human serum (for cross-validating clinical standard procedure and protein-A method), as well as dog, cat, rat, and monkey serum were labeled with protein-A from Staphylococcus aureus, binding the Fc portion of immunoglobulins of different species [26]. Plasma $(50 \mu \mathrm{l})$ and $25 \mu \mathrm{g}$ of FITC-conjugated protein-A (Sigma-Aldrich) were incumixture was then diluted to $250 \mu \mathrm{l}$ (PBS) and unbound FITC-Protein-A was removed using 100-kDa Amicon filter units (Sartorius, Göttingen, Germany), reconcentrating to $\sim 50 \mu 1$ [27]. NMDAR1-AB seropositivity was determined using Euroimmun assay combined with commercial monoclonal mouse NMDAR1-AB (114011; M68, SYSY, monoclonal mouse NMDAR1-AB (114011; M68, SYSY, Geling were rated "positive" (>98\% consensus of three investigators).

\section{Endocytosis assay}

Functional studies were conducted with sera following ammonium-sulfate precipitation of immunoglobulins [28] and dialysis (Slide-A-Lyzer® Mini Dialysis Units, 10,000 MWCO Plus Float, Thermo Fisher). To assess AB functionality, human IPSC-derived neurons were exposed to dialyzed serum [10]. For each species, arbitrarily selected seronegative $(N=1)$ and seropositive samples $(N=2-3)$ seronegative $(N=1)$ and seropositive samples $(N=2-3)$ were analyzed. Briefly, cells were precooled on ice and washed prior to incubation in cold HBSS with 1:50 diluted HBSS alone (negative control) for $30 \mathrm{~min} / 4^{\circ} \mathrm{C}$. After washing to reme (no their medi erlipste (a) exp 134308; Abce C C u ab134308, Abcam, Canbidge, UK, 1.100), followed by labeling with secondary donkey anti-mouse IgG (A10036; Life Technogies, Alexafluores46, 1.100). Neurons were fixed with ice-cold 4\% PFA and double stained with chicken anti-NeuN-AB (26606, SYSY, 1.500) and secondary donkey anti-chicken AB (703-546-155, Life Techologies, AlexaFlon $488,1.250)$. Nuclei were visualized using DAPI (Sigma-Aldrich, $0.01 \mu \mathrm{g} / \mathrm{ml}$ ). After PBS wash, coverslips were mounted on SuperFros - -Plus slides with Mowiol mounting media (Sigma-Aldrich). Confocal laserscanning microscopy was used to quantify NMDAR density at the membrane $(63 \times$ glycerol objective; TCS-SP5 Leica-Microsystems, Mannheim, Germany). From each coverslip, $\mathrm{Z}$ series of optical sections ( $0.5 \mu \mathrm{m}$ apart) covering the three-dimensional extension of neurons were acquired (sequential scanning mode, identical acquisition parameters). FIJI-ImageJ software [29] was used to randomly select $\mathrm{NeuN}^{+}$cells and determine soma profile. Fluorescence intensity/cell surface area (AlexaFluor546) was automatically measured as readout of NMDAR1 surface expression. After background subtraction, the mean intensity for each coverslip was determined and fluorescence intensity ratio $\left(37 / 4^{\circ} \mathrm{C}\right)$ was calculated.

\section{BBB-integrity testing}

BBB integrity of 12-month-old $\mathrm{ApoE}^{-/-}(N=5)$ and $\mathrm{ApoE}^{+/+}(N=5)$ mice was determined using two different fluorescent tracers, Evans blue ( $50 \mathrm{mg} / \mathrm{g}$ body weight) [30] and sodium fluorescein ( $200 \mathrm{mg} / \mathrm{g}$ body weight). A detailed description of this method will be published elsewhere [31]. Briefly, for tracer quantification in the brain at $4 \mathrm{~h}$ after
intravenous injection in the tail vein, animals were PBS intravenous injection in the tail vein, animals were PBS pected, immedintly frozen on dy ice, weighed, and sor- at $-80^{\circ} \mathrm{C}$. Tissue was lyophilized at $-56^{\circ} \mathrm{C}$ for $24 \mathrm{~h}$ under vacuum of $0.2 \mathrm{mBar}$ (Christ LMC-1-BETA-1-16, Osterode, Germany). For tracer extraction, hemispheres were incubated with shaking in $10 \mathrm{ml}$ formamide/mg brain at $57^{\circ} \mathrm{C}$ for $24 \mathrm{~h}$. Integrated density of tracer fluorescence was determined in triplicates on a fluorescent microscope (Observer Z2, Zeiss, Germany), equipped with AxioCamMRc3, 1 $\times$ Camera-Adapter, and ZEN2012 blue-edition software, recorded at $10 \times$ magnification (Plan-Apochromat software, recorded at $10 \times 10.45$ 27). Tracer concentration was standard curve and normalized to controls (set to 1).

\section{Mouse immunization}

Mice (12-month-old C57BL/6 littermates: $A p o E^{-1-} N=20$ and $\mathrm{ApoE}^{+/+} \mathrm{N}=23$; genders balanced) were immunized with a mixture of GluN1 extracellular peptides and/or chicken ovalbumin (Sigma-Aldrich), and emulsified in equal volume of complete Freund's Adjuvant (Мусоbacterium tuberculosis H37RA plus incomplete Freund's Adjuvant; Becton-Dickinson, Sparks, USA) at a final concentration of $1 \mathrm{mg} / \mathrm{ml}$ [32]. At the tail base, $50 \mu \mathrm{g}$ of GluN1 peptides and/or $20 \mu \mathrm{g}$ of ovalbumin were injected subcutaneously (each side one).

\section{Enzyme-linked immunosorbent assay (ELISA)}

Orbital sinus blood of immunized mice was stored as EDTA plasma at $-80^{\circ} \mathrm{C}$. ELISA plates ( 96 well) were coated with $0.5 \mu \mathrm{g}$ of GluN1 peptide mixture or $0.2 \mu \mathrm{g}$ of chicken ovalbumin in $50 \mu \mathrm{l} \mathrm{PBS} /$ well overnight at $4{ }^{\circ} \mathrm{C}$ and blocked with 5\% BSA/PBS (Carl Roth, Karlsruhe, Germany). Mouse plasma (1:1000 or 1:50,000 5\% BSA/PBS $50 \mu \mathrm{l} /$ well) was added for $2 \mathrm{~h}$ at RT. The signal was amplified with horseradish peroxidase-linked anti-IgG (Sigma-Aldrich), and 3,3',5,5'-Tetramethylbenzidine as colorimetric substrate (BD Biosciences, San Jose, USA). Absorbance at $450 \mathrm{~nm}$ was measured by microplate reader (Tecan-Trading AG, Männedorf, Switzerland).

\section{Basic behavioral screening}

The behavioral test battery was performed as described previously [33-36]. Starting at age 5 months, experimentally naïve $\mathrm{ApoE}^{-/-}$and $\mathrm{ApoE}^{+/+}$littermates underwent (during light phase) tests of anxiety, activity and exploratory behavior (elevated plus-maze, open field, hole-board), motor (rotarod, grip strength) and sensory function (visual cliff, olfaction, hearing hot plate), sensorimotor gating (pep based social preference, and cognitive, pheromoneIntellicage place/reversl leaning). Males and femas were tested separately.
Baseline and post MK-801 locomotion in the open field

The open-field apparatus consisted of a gray circular Perspex-arena $(120 \mathrm{~cm}$ diameter; wall height $25 \mathrm{~cm})$. Indirect white light illumination ensured constant light intensity of 120 lux in the center. Locomotion was measured using automated tracking software (Viewer2-Biobserve, Bonn, Germany). $A p o E^{-1-}$ and $A p o E^{+/+}$littermates received four baseline measurements preimmunization an post immunization (15 min each), the last followed by methyl-10,11-dihydro-5H-dibenzo[a,d]cyclohepten-5,10mine Aine Aling a a alding a a use-disen a nonch to induce psychosis-like hyperactivity in the open field (los of inhibition) [37. Direcly post injection, locomotor a fith ches over $120 \mathrm{~min}$ as $\%$ reference.

\section{Immunohistochemistry}

Mice were anesthetized with Avertin (2,2,2 Tribromoethanol, Sigma-Aldrich), and transcardially perfused with 4\% PFA/Ringer solution (Braun-Melsungen, Germany). Brains were removed, postfixed in 4\% PFA overnight at $4{ }^{\circ} \mathrm{C}$, and incubated in $30 \%$ sucrose/PBS for 2 days at $4{ }^{\circ} \mathrm{C}$. Brains were cryosectioned coronally into $30 \mu \mathrm{m}$ slices and stored in 25\% ethylene glycol and 25\% glycerol/PBS at $-20^{\circ} \mathrm{C}$. Frozen sections (three/mouse; rostral hippocampus), mounted on SuperFrost ${ }^{\circledR}$-Plus slides (Thermo Fisher, Waltham, USA), were used for cell quantification. For CD3 staining, sections were microwaved $3 \times$ for $4 \mathrm{~min}$ in citrate buffer $(1 \mathrm{mM}, \mathrm{pH} 6)$ and blocked with $5 \%$ normal horse serum (NHS), and $0.5 \%$ Triton X-100/ PBS for $1 \mathrm{~h}$ at RT. Incubation with rat anti-mouse CD3 (MCA1477; BioRad, Hercules, USA; 1:100) diluted in 5\% NHS, and $0.5 \%$ Triton X-100/PBS was performed for two nights $/ 4{ }^{\circ} \mathrm{C}$, followed by incubation with goat anti-rat AlexaFluor@647 (A-21247; Thermo Fisher, Schwerte, Germany; $1: 1000$ ) diluted in $5 \%$ NHS, and $0.5 \%$ Triton X100/PBS for $2 \mathrm{~h}$ at RT. For Iba1, GFAP, CD68, and MHCII staining, sections were blocked with 5\% NGS and/or 5\% NHS in $0.5 \%$ Triton X-100/PBS for $1 \mathrm{~h}$ at RT. Incubation with rabbit anti-mouse Iba1 (019-19741; Wako-Chemicals GmbH, Neuss, Germany; 1:1000), or mouse anti-mouse GFAP (NCL-GFAP-GA5; Novocastra-Leica, Newcastle upon Tyne, UK; $1: 500$ ), diluted in $3 \%$ NGS or $3 \%$ NHS, and $0.5 \%$ Triton X-100/PBS, was performed overnight, and incubation with rat anti-mouse CD68 (MCA1957GA BioRad GmbH, München, Germany, 1:400) and rat 
anti-mouse MHC-II (14-5321; eBioscience, San Diego, USA, 1:100) diluted in 3\% NGS and 3\% NHS, and $0.5 \%$ Triton X-100/PBS, was performed over two nights, all at $4{ }^{\circ}$ C. Incubation with secondary antibodies was performed with goat anti-rabbit AlexaFluor@555 (A-21428; Thermo Fisher; $1: 500$ ) diluted in 3\% NGS, $0.5 \%$ Triton X-100/PBS, or donkey anti-rabbit AlexaFluor®488 (A-21206; Thermo Fisher, 1:500) or donkey anti-mouse AlexaFluor488 (A21202; Thermo Fisher, 1:500) or goat anti-rat AlexaFluor@647 (A-21247: Thermo Fisher, 1.500), diluted in $3 \%$ NGS or $3 \%$ NHS, and $0.5 \%$ Triton X-100/PBS for $1.5 \mathrm{~h}$ at RT. Nuclei were counterstained with DAPI (SigmaAldrich, $0.01 \mu \mathrm{g} / \mathrm{ml}$ ) and sections were mounted using Aldrich, $0.01 \mu \mathrm{g} / \mathrm{ml}$ ) and sections were mounted using Aqua-Poly/Mount (Polysciences, Wanngton, USA) Tile scans of hippocampus were acquired using Leica-

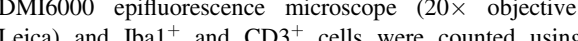
Leica) and Ibal and ${ }^{+} 3^{+}$cells were counted using CEFA ${ }^{+}$cells in the hippore Geapt cells in (a)

\section{Statistical analyses}

Statistical analyses were performed using SPSSv.17 (IBMDeutschland-GmbH, Munich, Germany) or Prism4 (GraphPad Software, San Diego, California, USA). Group differences in categorical and continuous variables were assessed using $\chi^{2}$, Mann-Whitney $\mathrm{U}$, or Student's $t$-tests depending on data distribution/variance homogeneity. ANOVA was employed as indicated in display item legends. All $p$-values are two tailed; significance is set to $p$ $<0.05$; data are presented as mean \pm S.E.M.

\section{Results}

Cross-validation of NMDAR1-AB detection methods

To determine NMDAR1-AB seropositivity in mammals other than humans, we had to validate the protein-A detection method [27]. For that, $N=72$ paired human serum and ventricular CSF samples, prospectively collected from random neurosurgical patients, were analyzed by the usual cell-based assay, employing specific secondary $\mathrm{AB}$ for all Ig classes. A total of $N=5$ sera turned out NMDAR1-AB positive (titers $\leq 1: 100 ; 3 \times \operatorname{IgM} ; 2 \times \operatorname{IgA}$; $0 \times \operatorname{IgG})$. Ventricular CSF samples were all negative. For cross-validation of NMDAR1-AB of the IgG class, we used serum of a seropositive stroke patient [8]. Application of protein-A method combined with double labeling for NMDAR1-AB M68 confirmed positive and negative results (Fig. 1a).
High seroprevalence of NMDAR1-AB across mammalian species

We next analyzed by protein-A method serum samples of dogs, cats, rats, baboons, and rhesus macaques. Strikingly, all mammalian species, independent of their respective life expectancy, show high NMDAR1-AB seropositivity (Fig. 1b). Mouse samples were analyzed using specific $A B$ against murine $\mathrm{IgA}$, IgM, and IgG. As known for humans [6], NMDAR1-AB of the IgG class were the rarest. Fo another cross-validation, all monkey samples $(N=100)$ were analyzed in blinded fashion by an independent lab (Bochum; using specific anti-monkey IgM). IgM-positive results coincided with the protein-A positivity by $>97 \%$ (76 of 78). The froction of protein-A positive but IgM(76 of NMDAR1-AB of IgA clas a d IGG class whe presents

Age-dependent NMDAR1-AB seroprevalence except for nonhuman primates and human migrants

All species revealed age dependence of NMDAR1-AB seroprevalence $\left(\chi^{2}\right.$ test; dogs: $\chi^{2}(1)=11.5, p=0.01$; cats $\chi^{2}(1)=4.8, p=0.03$; rats: $\chi^{2}(1)=9.5, p=0.002$; and mice: Fisher's exact test $p=0.032)$ as for humans $[5,8]$ with the exception of baboons $\left(\chi^{2}(1)=1.0, p=0.3\right)$, where already $>50 \%$ of young animals were seropositive. This surprising result made us investigate another monkey species, rhesus macaques, showing again high seroprevalence in old and young animals $\left(\chi^{2}(1)=0.2, p=0.6\right)$ (Fig. 1b). We wondered what the difference between humans, dogs, cats, mice, and rats, on one hand, and monkeys, on the other hand, could be, leading to loss of the usual age pattern regarding seroprevalence. Postulating that captivity/nondomestication of young monkeys might induce chronic life stress due to maladaptation to the environment, we investigated in a hypothesis-driven way whether young human migrants would display a similar increase in NMDAR1-AB seropositivity. Of the GRAS data collection, detailed information on migration was available in a subsample of $N$ $=970$ individuals. While nonmigrants show the typical age association of NMDAR1-AB seroprevalence $\left(\chi^{2}(1)=10.7\right.$ $p=0.001$ ), migrants do not $\left(\chi^{2}(1)=0.6, p=0.4\right)$ (Fig. 1c) Seroprevalence in young migrants is significantly higher as compared to young nonmigrants $\left(\chi^{2}(1)=5.381, p=0.020\right)$. In both monkey species and migrants, the IgM fraction still follows the expected age trend, while IgA seems to account for the early increase in NMDAR1-AB seroprevalence (Fig. 1c). Presentation of NMDAR1-AB by Ig class in the extended GRAS sample $(N=4933)$ with $N=4632$ of likely nonmigrants (available information less detailed) and $N=301$ known migrants, illustrates the
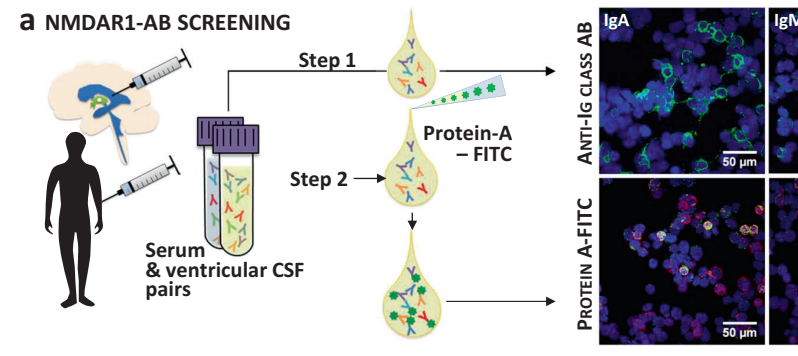

b NMDAR1-AB SEROPREVALENCE

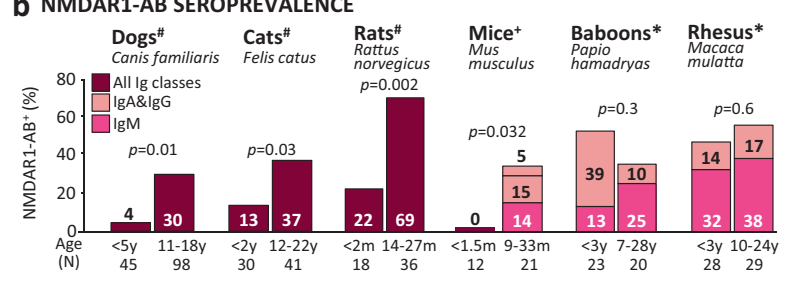

C NMDAR1-AB Human ${ }^{+}$Homo sapiens
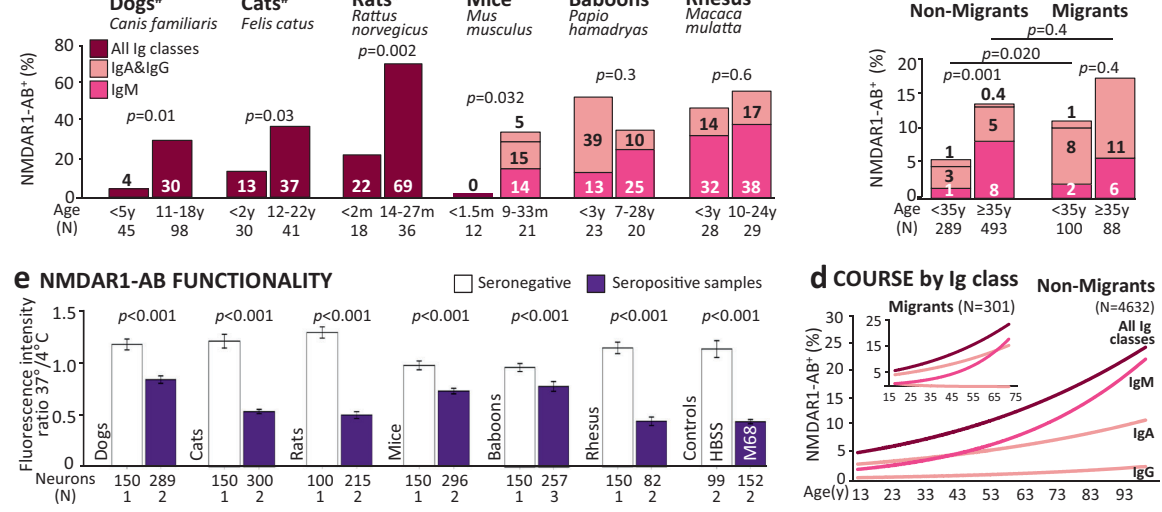
malian species. a Cross-validation of assays: paired serum and intraventricular CSF samples from neurosurgical patients were tested using a HEK293T cell-based clinical standard assay for NMDAR1-AB seropositivity (Euroimmun biochip). For step 1, fluorescently labeled
IgA-specific, IgM-specific, and IgG-specific secondary AB were used for method cross-validation (step 2), NMDAR1-AB seropositive and seronegative samples of each Ig class from step 1 were labeled with protein-A-FITC conjugate and tested for colocalization (yellow) of

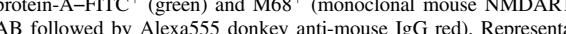
live pictures of both methods using the same seropositive samples (IgA, IgM, and IgG) are displayed on the right: upper row step 1/lower row step 2. b NMDAR-AB seropositivity (\%) of young and old

abnormal course of IgA vs. IgM/IgG seroprevalence over age in migrants (Fig. 1d).

Functionality of NMDAR1-AB from different mammalian species

To assess whether NMDAR1-AB of the tested species are functional, our endocytosis assay using IPSC-derived functional, our endocytosis assay using IPSC-derived
human cortical neurons [10] was employed. All positive mammals for all Ig classes combined ("protein-A-FITC/Euroimmun) or for individual classes ('Euroimmun; "protein-A-FITC/Euroimmu and cross-validation with Euroimmun/monkey IgM) presented in the bars; color codes used for consistency and kept also in $\mathbf{c}$ and $\mathbf{d}$; age
given in months ( $\mathrm{m}$ ) or years (y); $\chi^{2}$ or Fisher's exact test c $\mathrm{cMDAR}$ $A B$ seropositivity of subjects with migration (first and second generation) vs. nonmigration history (GRAS data collection); all Ig classes presented; age split at 35 years; $\chi^{2}$ test. $\mathbf{d}$ NMDAR1-AB course by Ig classes in serum over age groups in migrants vs. nonmigrants of the for IgA. eFunctionality testing of NMDAR1-AB in human IPSCderived cortical neurons: degree of internalization expressed as a ratio of fluorescence intensity measured at 37 and $4^{\circ} \mathrm{C}$; number of neurons and sera (N) given; Mann-Whitney U tes

sera provoked NMDAR1 internalization, verifying functionality (Mann-Whitney U; all $p<0.001$ ) (Fig. 1e).

BBB dysfunction but normal behavior of APOF $^{-I}$ mice

We next induced endogenous NMDAR1-AB formation in a mouse model of BBB dysfunction, $A p o E^{-1-}$ mice vs. WT littermates, $\mathrm{ApoE}^{+/+}$. Before that, we confirmed in 12 - 


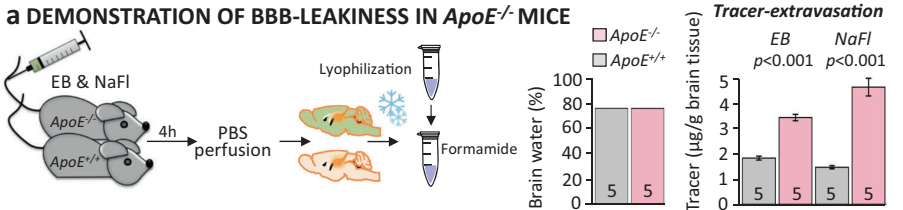

b EXPERIMENTAL OUTLINE

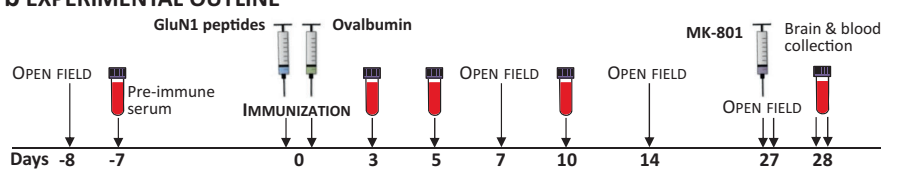

CIMMUNIZATION: PEPTIDES AND EFFICACY

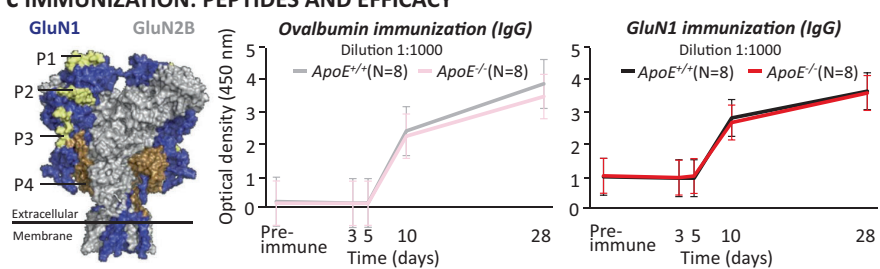

d OPEN FIELD ACTIVITY OF ApoE ${ }^{+/}$AND ApoE $\%$ MICE UPON MK-801 INJECTION
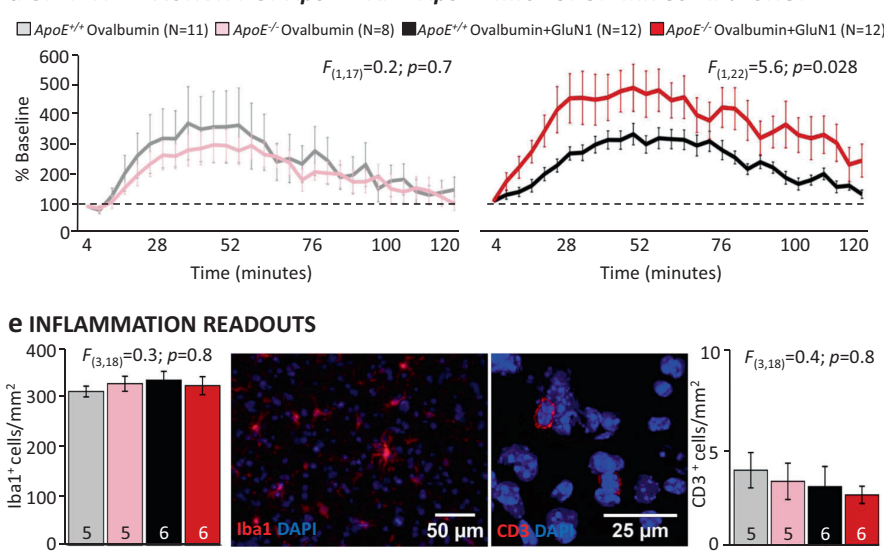

Fig. 2 Behavioral and morphological effects of endogenous NMDAR1- 801 injection on activity in the open field; results presented as \% change $\mathrm{AB}$ of the IgG class in a mouse model with open BBB. a Demonstration of $\mathrm{BBB}$ leakiness in $\mathrm{APOE}$, mice using an intravenously injected mixture of Evans blue (EB) and sodium woreschn (Naf). After brain Left: GluN1 peptides (P1-P4) located in the extracellular part of the receptor were used for immunization (compare Fig. 3); middle and right: Time course of anti-ovalbumin and anti-GluNI-AB (IgG) upo

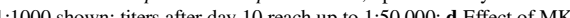

from baseline (first 4 min post MK-801 set to 100\%); no difference in MK-801-induced hyperactivity between genotypes after ovalbumi immunization (one-way repeated measures ANOVA. treatment $x$ group plateau, decline, and after-effect phases) unon MK-801 in $A p o E^{-1-}$ bu not $\mathrm{ApoE}^{+/+}$mice immunized against GluN1 (one-way repeated measures ANOVA: treatment $\times$ group interaction: $F_{(1,22)}=5.6 ; p=0.028$ ). e Quantification of $\mathrm{Iba}^{+}{ }^{+}$and $\mathrm{CD}_{3}{ }^{+}$cells in the hippocampus to asses Iba1 (left) and CD3 (right) stinings in the middle

\begin{tabular}{|c|c|c|c|c|c|c|c|c|}
\hline \multirow[b]{2}{*}{ Behavioral paradigms } & \multicolumn{4}{|l|}{ Males } & \multicolumn{4}{|l|}{ Females } \\
\hline & $\begin{array}{l}\begin{array}{l}\text { Age } \\
\text { (month) }\end{array} \\
\end{array}$ & $\begin{array}{l}\mathrm{ApoE}^{+/+} \\
(N)\end{array}$ & $\begin{array}{l}A p o E^{\prime-} \\
(N)\end{array}$ & $p$-value & $\begin{array}{l}\text { Age } \\
\text { (month) }\end{array}$ & $\begin{array}{l}\text { ApoE }^{+/+} \\
(N)\end{array}$ & $\begin{array}{l}A p o E^{-/} \\
(N)\end{array}$ & $p$-value \\
\hline \multicolumn{9}{|l|}{ Anxiety and activity } \\
\hline $\begin{array}{l}\text { Elevated plus-maze } \\
\text { (time open [\%]]) } \\
\text { Exploratory behavior }\end{array}$ & 5 & $\begin{array}{l}12.6 \pm 3.2 \\
(10)\end{array}$ & $\begin{array}{l}\begin{array}{l}19.5 \pm 4.0 \\
(10)\end{array} \\
\end{array}$ & $\begin{array}{l}p=0.14 \\
U=30.0\end{array}$ & 5 & $\begin{array}{l}17.5 \pm 2.9 \\
(13)\end{array}$ & $\begin{array}{l}14.8 \pm 1.3 \\
(11)\end{array}$ & $\begin{array}{l}p=0.98 \\
U=71.0\end{array}$ \\
\hline Hole-board (holes visited [\#]) & 5 & $\begin{array}{l}15.2 \pm 2.3 \\
(10)\end{array}$ & $\begin{array}{l}11.9 \pm 1.9 \\
(10)\end{array}$ & $\begin{array}{l}p=0.30 \\
t(18)=1.07\end{array}$ & 5 & $\begin{array}{l}\begin{array}{l}15.5 \pm 1.8 \\
(13)\end{array} \\
\text { (3) }\end{array}$ & $\begin{array}{l}15.6 \pm 2.9 \\
(13)\end{array}$ & $\begin{array}{l}p=0.96 \\
t(22)=0.96\end{array}$ \\
\hline \multicolumn{9}{|l|}{ Open-field } \\
\hline Locomotion $[\mathrm{m}]$ & 5 & $\begin{array}{l}31.8 \pm 1.7 \\
(10)\end{array}$ & $\begin{array}{l}32.7 \pm 1.5 \\
(10)\end{array}$ & $\begin{array}{l}p=\mathbf{0 . 7 0} \\
t(18)=0.39\end{array}$ & 5 & $\begin{array}{l}l_{(13)}^{2.7 \pm 1.3} \\
(13)\end{array}$ & $\begin{array}{l}43.7 \pm 3.2 \\
(13)\end{array}$ & $\begin{array}{l}p=0.76 \\
t(22)=0.31\end{array}$ \\
\hline \multicolumn{9}{|l|}{ Motor learning and coordination } \\
\hline $\begin{array}{l}\text { Rotarod day } 1 \text { (latency to } \\
\text { fall [s]) }\end{array}$ & 6 & $\begin{array}{l}89.3 \pm 11.6 \\
(10)\end{array}$ & $\begin{array}{l}130.0 \pm 15.3 \\
(10)\end{array}$ & $\begin{array}{l}p=0.06 \\
t(18)=2.01\end{array}$ & 5 & $\begin{array}{l}130.9 \pm 14.0 \\
(13)\end{array}$ & $\begin{array}{l}133.3 \pm 16.0 \\
(11)\end{array}$ & $\begin{array}{l}p=0.91 \\
t(22)=0.11\end{array}$ \\
\hline $\begin{array}{l}\text { Rotarod day } 2 \text { (latency to } \\
\text { fall [s]) }\end{array}$ & 6 & $\begin{array}{l}140.3 \pm 9.4 \\
(10)\end{array}$ & $\begin{array}{l}145.6 \pm 17.8 \\
(10)\end{array}$ & $\begin{array}{l}p=0.81 \\
t(18)=0.25\end{array}$ & 5 & 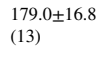 & 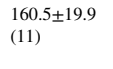 & $\begin{array}{l}p=0.5 \\
t(22)=0.69\end{array}$ \\
\hline $\begin{array}{l}\text { Muscle strength } \\
\text { Grip-strength [au] }\end{array}$ & 6 & $\begin{array}{l}110.2 \pm 5.4 \\
(10)\end{array}$ & $\begin{array}{l}122.0 \pm 5.0 \\
(10)\end{array}$ & $\begin{array}{l}p=0.15 \\
t(18)=1.52\end{array}$ & 6 & $\begin{array}{l}108.8 \pm 3.0 \\
(13)\end{array}$ & $\begin{array}{l}115.1 \pm 4.4 \\
(11)\end{array}$ & $\begin{array}{l}p=0.26 \\
t(22)=1.16\end{array}$ \\
\hline \multicolumn{9}{|l|}{ Heatpain perception } \\
\hline Hot-plate (latency to lick [s]) & 5 & $\begin{array}{l}12.8 \pm 0.4 \\
(10)\end{array}$ & $\begin{array}{l}11.9 \pm 0.7 \\
(10)\end{array}$ & $\begin{array}{l}p=\mathbf{0 . 2 2} \\
t(18)=1.26\end{array}$ & 5 & $\begin{array}{l}13.7 \pm 0.5 \\
(12)\end{array}$ & $\begin{array}{l}12.4 \pm 0.5 \\
(10)\end{array}$ & $\begin{array}{l}p=\mathbf{0 . 1 5} \\
t(20)=1.5\end{array}$ \\
\hline \multicolumn{9}{|l|}{ Vision } \\
\hline $\begin{array}{l}\text { Visual-cliff (time on } \\
\text { "air" side [\%]) } \\
\text { olfaction }\end{array}$ & 5 & $\begin{array}{l}26.5 \pm 7.2 \\
(10)\end{array}$ & $\begin{array}{l}22.0 \pm 5.6 \\
(10)\end{array}$ & $\begin{array}{l}p=0.85 \\
U=47.0\end{array}$ & 5 & $\begin{array}{l}21.7 \pm 5.1 \\
(13)^{2}\end{array}$ & $\begin{array}{l}29.0 \pm 3.9 \\
(11)\end{array}$ & $\begin{array}{l}p=0.13 \\
U=45.0\end{array}$ \\
\hline $\begin{array}{l}\text { Buried food-test (latency to find } \\
\text { cookie ss]) }\end{array}$ & 5 & $\begin{array}{l}59.4 \pm 9.2 \\
(10)\end{array}$ & $50.6 \pm 8.5(9)$ & $\begin{array}{l}p=0.52 \\
t(17)=0.66\end{array}$ & 5 & $\begin{array}{l}47.8 \pm 12.9 \\
(12)\end{array}$ & $\begin{array}{l}50.7 \pm 10.7 \\
(11)\end{array}$ & $\begin{array}{l}p=0.87 \\
t(21)=0.16\end{array}$ \\
\hline $\begin{array}{l}\text { Hearing } \\
\text { Acoustic startle at } 65 \mathrm{~dB}[\mathrm{AU}]\end{array}$ & 6 & $\begin{array}{l}0.5 \pm 0.04 \\
(10)\end{array}$ & $\begin{array}{l}0.5 \pm 0.04 \\
(10)\end{array}$ & $\begin{array}{l}p=\mathbf{0 . 5 3} \\
F(1,18)=0.42\end{array}$ & 8 & $\begin{array}{l}0.4 \pm 0.1 \\
(13)\end{array}$ & $\begin{array}{l}0.5 \pm 0.04 \\
(11)\end{array}$ & $\begin{array}{l}p=0.19 \\
F(1,22)=1.82\end{array}$ \\
\hline Acoustic startle at $120 \mathrm{~dB}[\mathrm{AU}]$ & & $4.5 \pm 1.0(10)$ & $4.8 \pm 1.0(10)$ & & & $\begin{array}{l}3.3 \pm 0.5 \\
(13)\end{array}$ & $\begin{array}{l}4.2 \pm 0.6 \\
(11)\end{array}$ & \\
\hline \multicolumn{9}{|l|}{ Sensorimotor gating } \\
\hline Mean pre-pulse inhibition [\%] & 6 & $\begin{array}{l}44.8 \pm 6.7 \\
(10)\end{array}$ & $\begin{array}{l}40.6 \pm 7.4 \\
(10)\end{array}$ & $\begin{array}{l}p=0.69 \\
F(1,18)=0.16\end{array}$ & 8 & $\begin{array}{l}57.7 \pm 4.1 \\
(13)\end{array}$ & $\begin{array}{l}50.4 \pm 6.3 \\
(11)\end{array}$ & $\begin{array}{l}p=0.35 \\
F(1,22)=0.91\end{array}$ \\
\hline \multicolumn{9}{|l|}{ Pheromone-based social preference } \\
\hline $\begin{array}{l}\text { Time spent in pheromone } \\
\text { box [s] }\end{array}$ & & & & & 15 & $\begin{array}{l}1213 \pm 50.8 \\
(12)\end{array}$ & $\begin{array}{l}1115 \pm 83.7 \\
(12)\end{array}$ & $\begin{array}{l}p=\mathbf{0 . 3 3} \\
t(22)=1.0\end{array}$ \\
\hline Time spent in control box [s] & & & & & & $\begin{array}{l}780.5 \pm 75.4 \\
(12)\end{array}$ & $\begin{array}{l}751.1 \pm 83.5 \\
(12)\end{array}$ & $\begin{array}{l}p=0.84 \\
t(22)=0.21\end{array}$ \\
\hline \multicolumn{9}{|c|}{ Cognitive performance in Intellicage } \\
\hline $\begin{array}{l}\text { Place-learning }[\% \text { target corner } \\
\text { visits }]^{2}\end{array}$ & & & & & 15 & $\begin{array}{l}34.2 \pm 1.3 \\
(12)\end{array}$ & $\begin{array}{l}34.2 \pm 1.8 \\
(13)\end{array}$ & $\begin{array}{l}p=0.76 \\
U=72.0\end{array}$ \\
\hline $\begin{array}{l}\text { Reversal-learning [\% target } \\
\text { corner visits] }\end{array}$ & & & & & & $\begin{array}{l}34.2 \pm 1.3 \\
(12)\end{array}$ & $\begin{array}{l}34.2 \pm 1.8 \\
(13)\end{array}$ & $\begin{array}{l}p=0.17 \\
U=52.0\end{array}$ \\
\hline
\end{tabular}

\section{as previously described in Netrakanti et al. 2015}

Note: All data in the table are mean + S.EM.

month-old mice (age of immunization) BBB leakiness using $\quad-10.66, \quad p<0.001 ; \quad$ NaFl: $\quad t(8)=-8.97, \quad p<0.001)$ two fluorescent tracers. While brain water content was (Fig. 2a). We wondered whether this compromised similar in both genotypes, pointing aganst inflommation, $\mathrm{BBB}$ would by itself lead to behavioral abnormalitis in

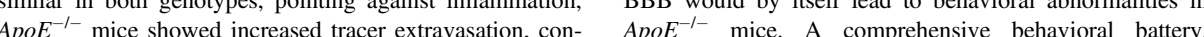
firming $\mathrm{BBB}$ dysunction (Studnis thest: $\mathrm{EB}: t(8)=$ 
motor and sensory function, sensorimotor gating,
pheromone-based social preference, and cognitive and MHCII, was essentially negative and identical mance did not reveal any differences between genotypes reveal any appreciable density increase in the (Table 1).

\section{Immunization of $A p O E^{-1}$ and $\mathrm{ApOE}^{+/+}$mice against} NMDAR1-peptides

To explore whether endogenously formed NMDAR1-AB would lead to measurable behavion and morphological effects, we immunized 12-month-old $A p o E^{-/-}$and $A p o E^{+/ 4}$ littermates against four peptides of the exp $\mathrm{ApOE}^{+/ 4}$ littermates against four peptides of the extracellular G369) G369) and ovalbumin or against ovalbumin alone as immunization control (Fig. 2b-c). GluN1 shows $>99 \%$ species, with immunizing peptides being $100 \%$ homologous (Fig. 3). Immination peptes being $100 \%$ homologous

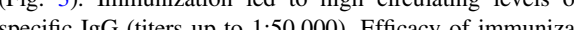
specific Ig (thers up to $1.50,000$ ). Eficacy of immuniza-

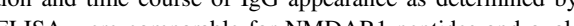
ELISA were conparable for NMDARI-peptides and ovalbumin a cross gentypes, haking a simple bossting effect of cell clones rather improbable (Fig. 2c).

\section{Psychosis-related behavior of $\mathrm{ApoE}^{-1-}$ mice upon}

\section{MK-801 challenge}

Open-field tests measuring baseline preimmunization an postimmunization locomotion did not reveal any differences between genotypes and/or immunization groups (Fig. 2b; not shown). After 4 weeks, the endogenously formed NMDAR1-AB of the IgG class induced strong hyperactivity (psychosis-like symptoms [37]) upon MK-801 challenge in $A p o E^{-/-}$mice only. In contrast, $A p o E^{+/}$ mice behaved comparably to ovalbumin-only immunized mice of both genotypes (Fig. 2d; all $p>0.5$ ). Thus, an open $\mathrm{BBB}$ together with sufficiently high titers of $\mathrm{AB}$ (to reach threshold loss of NMDAR1 surface expression) is a prerequisite for the observed behavioral perturbation upon MK-801.

\section{No inflammation in hippocampus of immunized} $\mathrm{ApoE}^{-/-}$and $\mathrm{ApoE}^{+/+}$mice

Immunohistochemistry did not show any evidence of inflammation in either genotype and/or immunization group. Numbers of $\mathrm{Iba}^{+}{ }^{+}$and $\mathrm{CD}^{+}$cells as markers of microglia and $\mathrm{T}$ cells, respectively, were comparable for total hippocampus (one-way ANOVA: Iba1: $F(3,18)=0.3$; $p=0.8 ; \mathrm{CD} 3: F(3,18)=0.4 ; p=0.8$ ) (Fig. $2 \mathrm{e}$ ) and for all hippocampal subfields separately (all $p$-values $>02$. not (hown). Also, staining for microglial activity marker, reveal any appreciable density increase in the shown).

\section{Discussion}

The present work demonstrates high seroprevalence of Thetional NMDAR $1-A B$ of all Ig classes across mammals, indicating that these $A B$ are part of a pre-existing autoimmune repertoire [11-17]. As in humans, NMDAR1-AB of the $\mathrm{IgG}$ class are the least frequent $[6,20]$. The age related up to $>50 \%$ NDDAR $A B$ seropositity is inde(he a cering a certain environment tiggens NMDAR1-AB formation. con dective

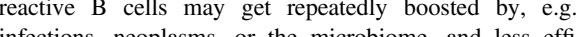
cienty suppressed over a lifespan likely owing to a gradua loss of immune tolerance upon aging $[14$

Unextedy, we find he age-dependence lost in nonhuman primates and in human migrants that all display an early-life rise in NMDARI-AB seropositivity, mainly of IgA. The intriguing possibility that chronic life stress, known to be present in human migrants [38] and animals in captivity [39], acts as a trigger of early NMDAR1-AB formation is worth pursuing experimentally in the future. A large proportion of migrants in our human samples are tionally support our chronic stress hypothesis since migrato mental disease [40]. Further studies should screen wildlife monkeys and species in zoos for NMDAR1-AB Experimental confirmation of our findings provided, NMDARI-AB (IgA) may even serve as stress markers. In fact, earlier reports show that total serum-Ig of all classes, most prominently IgA, respond to psychological stress [41] NMDAR1-AB might thus belong to a set of stress-boosted AB. Interestingly, we also find accumulated seroprevalence of 23 other brain-directed $\mathrm{AB}[6]$ in young migrants vs. nonmigrants increased (data not shown), suggesting a gloEarlier work has shown that $\mathrm{AB}$ against brain antigens in general are common among mammals [42], but no study has so far systematically screened nonhuman mammals for NMDAR1-AB. As an exception, a recent report described "anti-NMDAR1 encephalitis" in the young polar bear Knut pendent of the respective species' life expectancy, is inde

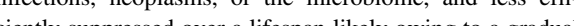
suffering from neuropsychiatric illness. This may addition is recognized as an environmental stressor predisposing bal inducer role of chronic stress in humoral autoimmunity. [27]. Based on the present findings, Knut may have

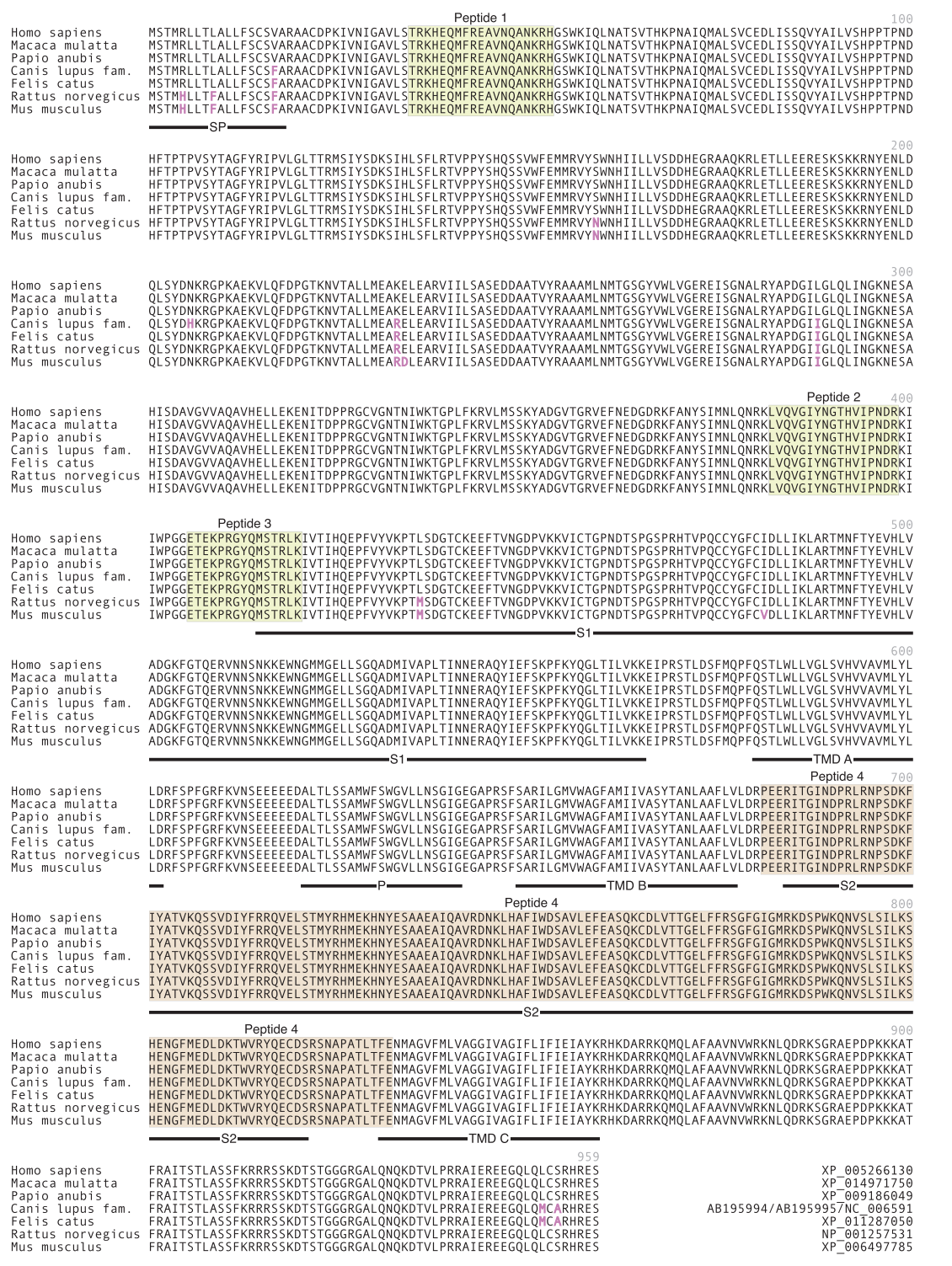
Fig. 3 Alignment of GluN1-1b receptor amino acid sequence across all
mammalian species tested. Regions containing the four peptide
sequences (peptides 1-4: P1: AA35-53, P2: AA361-376, P3: ment are highlighted in yellow ase light bon he domain, $T M D$ A transmembrane domain A, 
comparable to monkey species investigated here - that are affected by chronic early-life stress, inducing NMDAR1AB seropositivity. Pre-existing NMDAR1-AB of this bear may have ultimately shaped the clinical picture of an encephalitis of unexplained origin (likely infectious according to the zoo's pathology reports) where an epileptic seizure led to drowning [27].

This interpretation is supported by our novel autoimmune model, namely, mice immunized agains NMDAR1-peptides. Even high titers of endogenously formed NMDAR1-AB (IgG; up to 1.50,000) that insty psychosis-like behavior upon MK-801 chatenduce Apo $E^{-1-}$ mice, with here-confirmed open $\mathrm{BBB}$, do not to any apprecibe to any appreciablo sign of encephavis. be bin is of major importance for clici [14]. Fo the brain is of mor mpor AB inful $A B$ infusions into the hippocanpus on learning and memory in mice [43], and ohers for dis insed NMDARI-AB

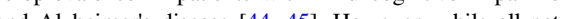
a d rally occuning NMDARI-AB that have pathogenic potential irrespective of epitope and $\mathrm{Ig}$ class [10], and upon entry to the brain (or via intrathecal production) can shape brain functions in the sense of NMDAR antagonism, only a fraction of individuals happens to have underlying encephalitis of various etiologies, which is then called anti-NMDAR encephalitis. The highly variable neuropathology and response to immunosuppression of this condition $[2,3,46]$ may point to a broad range of possible encephalitogenic mechanisms (from infection to oncology or genetics) which need to be diagnosed and specifically treated [14].

Even though it is unclear how NMDAR1-AB are generated by chronic stress, it should be considered that NMDAR1 are not only expressed in the brain but also by peripheral organs and tissues, including adrenal gland and gut [47] which may be involved in triggering NMDARI-AB formation but may also be functionally modulated by them. Since NMDAR antagonists are increasingly recognized as antidepressant, anxiolytic, and anti-inflammatory agents [48-52], we speculate that stress-induced NMDAR1-AB could serve as endogenous stress protectants. Remarkably, also in stroke, NMDAR1$\mathrm{AB}$ can be protective [8].

In conclusion, the widespread occurrence of NMDAR1$\mathrm{AB}$ across mammals, as well as the failure of even high titers of endogenously formed NMDAR1-AB of the IgG class to induce any signs of brain inflammation should lead neuropsychiatric disease including encephalitis.

Acknowledgements This work was supported by the Max Planck
Society, the Max Planck Förderstiftung, the DFG (CNMPB),
EXTRABRAIN EU-FP7, and the Niedersachsen-Research Network on Neuroinfectiology (N-RENNT). The authors thank all subjects
for participating in the study, and the many colleagues who have for participating in the study, and the many colleagues who have
contributed over the past decade to the extended GRAS data contributed
collection.

Author contributions Concept, design, and supervision of the study
HE; Data acquisition/analysis/interpretation: HP, BO, ED, DT, MM HE; Data acquisition/analysis/interpretation: HP, BO, ED, DT, MM,
JS, JW, DW, CKS, AR, KS, RT, KMR, StB, YAK, HM, MB, WS JS, JW, DW, CKS, AR, KS, RT, KMR, SB,
GS, FJK, RM, SB, KAN, JKK, MH, FL, and HE; Drafting manuscript: $\mathrm{HE}$, with the help of $\mathrm{BO}$ and $\mathrm{HP}$; Drafting display items: $\mathrm{HE}$ and $\mathrm{BO}$ with the help of HP, MM, DT, and JS. All authors read and approved

Compliance with ethical standards

Conflict of interest WS is a member of the board and holds stocks in GmbH. The remaining authors declare that they hye no conflict of interest.

Open Access This article is licensed under a Creative Common Attribution-NonCommercial-ShareAlike 4.0 International License, wich pechls any non-commercial use, sharing, adaptation, distribuappropriate credit to the original author(s) and the source, provide link to the Creative Commons license, and indicate if changes were made. If you remix, transform, or build upon this article or a part thereof, you must distribute your contributions under the same license
as the original. The images or other third party material in this article 列 cated otherwise in a credit line to the material. If material is no included in the article's Creative Commons license and your intended use is not permitted by statutory regulation or exceeds the permitted holder. To view a copy of this license, visit http //creativecoprigh org/licenses/by-nc-sal/4.0/.

\section{References}

1. Dalmau J, Tuzun E, Wu HY, Masjuan J, Rossi JE, Voloschin A, et al. Paraneoplastic anti-N-methyl-D-aspartate receptor encephalitis associated with ovath
2007;61:25-36. 2. Dalmau J, Gleichman AJ, Hughes EG, Rossi JE, Peng X, Lai M, et al. Anti-NMDA-receptor encephalitis: case series and analysis of the effects of antibodies. Lancet Neurol. 2008;7:1091-8.
3. Dalmau J, Lancaster E, Martinez-Hernandez E, Rosenfeld MR, Dalmau J, Lancaster E, Martinez-Hernandez E, Rosenfeld MR,
Balice-Gordon R. Clinical experience and laboratory investigaBalice-Gordon R. Clinical experience and laboratory investiga-
tions in patients with anti-NMDAR encephalitis. Lancet Neurol.
2011;10:63-74.

4. Titulaer MJ, McCracken L, Gabilondo I, Armangue T, Glaser C, lizuka T, et al. Treatment and prognostic factors for long-term outcome in patients with anti-NMDA receptor ence 2013;12:157-65.

5. Hammer C, Stepniak B, Schneider A, Papiol S, Tantra M, Begemann M, et al. Neuropsychiatric disease relevance of circulating anti-NMDA receptor autoantibodies depends on bloo-
brain barrier integrity. Mol Psychiatry. 2014:19:1143-9.9. brain barrier integrity. Mol Psychiatry. 2014;19:1143-9.
6. Dahm L, Ott C, Steiner J, Stepniak B, Teegen B, Saschenbrecker $\mathrm{S}$, et al. Seroprevalence of autoantibodies against brain antigens in health and disease. Ann Neurol. 2014;76:82-94.
7. Steiner J, Teegen B, Schiltz K, Bernstein HG, Stoecker W, Bogerts B. Prevalence of N-methyl-D-aspartate receptor auto-
antibodies in the peripheral blood: healthy control samples antibodies in the peripheral blood: healthy control samples
revisited. JAMA Psychiatry. 2014;71:838-9.

revisited. JAMA Psychiatry. 2014;71:838-9.
8. Zerche M, Weissenborn K, Ott C, Dere E, Asif AR, Worthmann $\mathrm{H}$, et al. Preexisting serum autoantibodies against the NMDAR
subunit NR1 modulate evolution of lesion size in acute ischemic stroke. Stroke. 2015;46:1180-6.

Castillo-Gomez E, Kastner A, Steiner J, Schneider A, Hettling B, Poggi G, et al. The brain as immunoprecipitator of serum auto-
antibodies against N-Methyl-D-aspartate receptor subunit NR1. Ann Neurol. 2016;79:144-51.

10. Castillo-Gomez E, Oliveira B, Tapken D, Betrand S, KleinSchmidt C, Pan H et al. All naturally occurring autoantibodie against the NMDA receptor subunit NR1 have pathogenic potential irrespective of epitope
Psychiatry. 2016;22:1776-178.

11. Abramson J, Husebye ES. Autoimmune regulator and self-tolerance-molecular and clinical aspects. Immunol Rev. 2016;

12. Cohen I, Young D. Autoimmunity, microbial immunity and the

13. Coutinho A, Kazatchkine MD, Arrameas S. Natural auto-

13. Catibodies. Curr Opin Immunol. 1995;7:812-8.
14. Ehrenreich H. Autoantibodies against the N-methyl-d-aspartate Ehrenreich H. Autoantibodies against the N-methyl-d-aspartate
receptor subunit NR1: untangling apparent inconsistencies for
clinical practice. Front Immunol. 2017:8: 181 . 15. Lobo PI. Role of natural autoantibodies and natural $\mathrm{IgM}$ anti-
leucocyte autoantibodies in health and disease. Front Immunol. 2016;7:198.

16. Mader S, Brimberg L, Diamond B. The role of brain-reactive autoantibodies in brain
Immunol. 2017:8:1101.

17. Nguyen TT, Baumgarth N. Natural IgM and the development of b cell-mediated autoimmune diseases. Crit Rev Immunol. 2016 36:163-77,

18. Coutinho E, Harrison P, Vincent A. Do neuronal autoantibodies cause psychosis? A neuro
chiatry. 2014;75:269-75.

19. Diamond B, Huerta P, Mina-Osorio P, Kowal C, Volpe B. Losing your nerves? Maybe it's the antibodies. Nat Rev Immunol

20. Hammer C, Zerche M, Schneider A, Begemann M, Nave KA, Ehrenreich H. Apolipoprotein E4 carrier status plus circulating
anti-NMDAR1 autoantibodies: association with schizoaffective anti-NMDAR1 autoantibodies: association
disorder. Mol Psychiatry. 2014;19:1054-6.

21. Kreye J, Wenke NK, Chayka M, Leubner J, Murugan R, Maier N et al. Human cerebrospinal fluid monoclonal N-methyl-Daspartate receptor autoantibodies are sufficient for encephalitis

22. Begemann MGS, Papiol S, Malzahn D, Krampe H, Ribbe K, et al. Modification of cognitive performance in schizophrenia by com-
plexin 2 gene polymorphisms. Arch Gen Psychiatry. 2010;67:

23. Ribbe. K. drichs H, Begemann M, Grube S, Papiol S, Kästner. A, et al. The cross-sectional GRAS sample: a comprehensive
phenotypical data collection of schizophrenic patients. BMC Psychiatry. 2010;10:0-20.

24. American Psychiatric Association. (2000) Diagnostic and statistical manual of mental disorders.

5. Wandinger KP, Saschenbrecker S, Stoecker W, Dalmau J. AntiNMDA-receptor encephalitis: a severe, multistage, treafa disorder presenting with psychosis. J Neuroimmunol. 2011,231:86-91.
6. Boyle MDP, Reis KJ. Bacterial Fc receptors. Nat Biotechnol. 1987:5:697-703.
27. Pruss H, Leubner J, Wenke NK, Czirjak GA, Szentiks CA, Greenwood AD. Anti-NMDA receptor encephalitis in the polar bear (Ursus maritimus) Knut. Sci Rep. 2015;5:1-7.
Toyka KBD, Pestronk A, Kao I. Myasthenia gravis: passive 28. Toyka KBD, Pestronk A, Kao I. Myasthenia gravis: passive
transfer from man to mouse. Science. 1975;190:397-9.

29. Schindelin J, Arganda-Carreras I, Fisise $E$, Kaynig V, Longair $M$,
Pietzch T, et al. Fiji: an open-source platform for biologicalimage analysis. Nat Methods. 2012;9:676-82.

30. Berghoff SA, Gerndt N, Winchenbach J, Stumpf SK, Hosang L, Odoardi $F$, et al. Dietary cholesterol promotes repair of
lesions in the adult brain. Nat Commun. 2017:8:1-15.

31. Berghoff SA, Düking T, Spieth L, Winchenbach J, Stumpf SK, Gerndt $\mathrm{N}$, et al. Blood-brain barrier hyperpermeability precedes
demyelination in the cuprizone model. Acta Neuropathol Comdemyelination in
mun. 2017:5:94.

32. Wust S, van den Brandt J, Tischner D, Kleiman A, Tuckermann $\mathrm{JP}$, Gold R, et al. Peripheral T cells are the therapeutic targets of
glucocorticoids in experimental autoimmune encephalomyelitis. J Immunol. 2008:180:8434-43.

Dere E, Dahm L, Lu D, Hammerschmidt K, Ju A, Tantra M, et al. Heterozygous ambra1 deficiency in mice: a genetic trait with
autism-like behavior restricted to the female gender. Front Behav Neurosci. 2014;8:181.

34. Dere E, Winkler D, Ritter C, Ronnenberg A, Poggi G, Patzig J, et al. Gpm6b deficiency impairs sensorimotor gating and modulates the behavioral response to a 5-HT2A/C receptor agonist.
Behav Brain Res. 2015;277:254-63.

5. Netrakanti PR, Cooper BH, Dere E, Poggi G, Winkler D, Brose N, et al. Fast cerebellar reflex circuitry requires synaptic vesicle priming by munc13-3. Cerebellum. 2015;14:264-83.
36. Winkler D, Daher F, Wustefeld L, Hammerschmidt K, Poggi G, Seelbach A et al. Hypersocial behavior and biological redundancy
in mice with reduced expression of PSD95 or PSD93. Behav Brain Res. 2017. http:///doi.org/10.1016/j.bbr.2017.02.011 (e-pub ahead of print).

37. Radyushkin K, El-Kordi A, Boretius S, Castaneda S, Ronnenberg A, Reim K, et al. Complexin2 null mutation requires a 'second hit' for induction of phenotypic changes relevant to schizophrenia
Genes Brain Behav. 2010;9:592-602.

. Akdeniz C, Tost H, Streit F, Haddad L, Wust S, Schafer A, et al. Neuroimaging evidence for a role of neural social stress proces-
sing in ethnic minority-associated environmental risk. JAMA Psychiatry. 2014;71:672-80.
Jacobson SL, Ross SR, B

Jacobson SL, Ross SR, Bloomsmith MA. Characterizing abnormal behavior in a large population of zoo-housed chimpanzees:
prevalence and potential influencing factors. PeerJ. 2016;4 e2225.
Stepniak B, Papiol S, Hammer C, Ramin A, Everts S, Hennig L, et al. Accumulated environmental risk determining age at schi-
zophrenia onset: a deep phenotyping-based study. Lancet Psyzophrenia onset: a deep
chiatry. 2014;1:444-53.

41. Maes M, Hendriks D, Gastel AV, Demedts P, Wauters A, Neels $\mathrm{H}$, et al. Effects of psychological stress on serum immunoglobulin, complement and acute phase protein concentrations
in normal volunteers. Psychoneuroendocrinology. 1997;2:

42. Nagele EP, Han M, Acharya NK, DeMarshall C, Kosciuk MC Nagele RG. Natural IgG autoantibodies are abundant and ubiquitous in human sera, and their number is influenced by age,

Garcia E, Aguilar E, et al. Human N-methyl D-aspasta J, Martinantibodies alter memory and behaviour in mice. Brain. 2015;138:94-109.

Busse S, Brix B, Kunschmann R, Bogerts B, Stoecker W, Busse 
49. Abdallah CG, Adams TG, Kelmendi B, Esterlis I, Sanacora G,

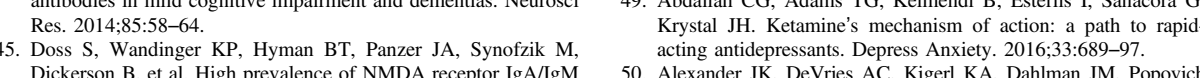
$\begin{array}{ll}\text { Dickerson B, et al. High prevalence of NMDA receptor IgA/IgM } & \text { 50. Alexander JK, DeVries AC, Kigerl KA, Dahlman JM, Popovich } \\ \text { antibodies in different dementia types. Ann Clin Transl Neurol. } & \text { PG. Stress exacerbates neupathic pain via gl }\end{array}$ PG. Stress exacerbates neuropathic pain via glucocorticoid and

46. Tuzun E, Zhou L, Baehring JM, Bannykh S, Rosenfeld MR, 51. Rubio-Casillas A, Fernandez-Guasti A. The dose makes the poiDalmau J. Evidence for antibody-mediated pathogenesis in anti- son: from glutamate-mediated neurogenesis to neuronal atrophy and depression. Rev Neurosci. 2016,27:599-622.
52. Nair $A$. Hunzeker J. Bonneau RH. Modulation of microgli and Nu J, Li XH Li YJ Glutme in peripheral organs: biology and 52 . Nair A, Huncer J, Bonneau RH. Modulation of microglia and induced herpes simplex virus type-1 encephalitis. Brain Behav 48. Gerhard DM, Wohleb ES, Duman RS. Emerging treatmen mechanisms for depression: focus on glutamate and synaptic Immun. 2007;21:791-806.

\section{Affiliations}

Hong Pan ${ }^{1} \cdot$ Bárbara Oliveira ${ }^{1} \cdot$ Gesine Saher ${ }^{2} \cdot$ Ekrem Dere $^{1} \cdot$ Daniel Tapken $^{3} \cdot$ Marina Mitjans $^{1} \cdot$ Jan Seidel ${ }^{1}$.

Janina Wesolowski ${ }^{1} \cdot$ Debia Wakhloo $^{1} \cdot$ Christina Klein-Schmidt $^{3} \cdot$ Anja Ronnenberg $^{1} \cdot$ Kerstin Schwabe $^{4}$.

Ralf Trippe $^{3} \cdot$ Kerstin Mätz-Rensing $^{5} \cdot$ Stefan Berghoff ${ }^{2} \cdot$ Yazeed Al-Krinawe $^{4} \cdot$ Henrik Martens $^{6} \cdot$ Martin Begemann $^{1}$

Winfried Stöcker ${ }^{7} \cdot$ Franz-Josef Kaup $^{5} \cdot$ Reinhard Mischke $^{8} \cdot$ Susann Boretius $\oplus^{9} \cdot$ Klaus-Armin Nave $^{2,10}$

Joachim K. Krauss ${ }^{4} \cdot$ Michael Hollmann $\odot^{3} \cdot$ Fred Lühder $^{11}$. Hannelore Ehrenreich ${ }^{1,10}$

Clinical Neuroscience, Max Planck Institute of Experimental Medicine, Göttingen, Germany

2 Department of Neurogenetics, Max Planck Institute of Experimental Medicine, Göttingen, Germany

Department of Biochemistry I-Receptor Biochemistry, Ruhr University, Bochum, Germany

4 Department of Neurosurgery, Hannover Medical School,

Hannover, Germany

5 Department of Pathology, Leibniz Institute for Primate Research,

6 Synaptic Systems GmbH, Göttingen, Germany
Institute for Experimental Immunology, affiliated to Euroimmun, Lübeck, Germany

8 Small Animal Clinic, University of Veterinary Medicine, Hannover, Germany

9 Functional Imaging Laboratory, Leibniz Institute for Primate

10 DFG Research Center for Nanoscale Microscopy and Molecular Physiology of the Brain (CNMPB), Göttingen, Germany

11 Department of Neuroimmunology, Institute for Multiple Sclerosis Research and Hertie Foundation, University Medicine Göttingen, Göttingen, Germany 
APPENDIX 


\section{A.2 List of publications}

Seidel, J.* , Bockhop, F.*, Mitkovski, M.*, Martin, S., Ronnenberg, A., Krueger-Burg, D., Schneider, K., Röhse, H., Wüstefeld, L., Cosi, F., Bröking, K., Schacht, A., Ehrenreich, H. (2020). Vascular response to social cognitive performance measured by infrared thermography: A translational study from mouse to man. FASEB BioAdvances, 2, 18-32.

Seidel, J. \& Ehrenreich, H. (2018). Frühe Risikoakkumulation und Kriminalität im Erwachsenenalter. Schweizer Zeitschrift für Psychiatrie \& Neurologie, 04, 7-9.

Mitjans, M.*, Seidel, J.*, Begemann, M.*, Bockhop, F., Moya-Higueras, J., Bansal, V., Wesolowski, J., Seelbach, A., Ibáñez, M. I., Kovacevic, F., Duvar, O., Fañanás, L., Wolf, H.U., Ortet, G., Zwanzger, P., Klein, V., Lange, I., Tänzer, A., Dudeck, M., Penke, L., Tebartz van Elst, L., Bittner, R. A., Schmidmeier, R., Freese, R., Müller-Isberner, R., Wiltfang, J., Bliesener, T., Bonn, S., Poustka, L., Müller, J. L., Arias, B., Ehrenreich, H. (2018). Violent aggression predicted by multiple pre-adult environmental hits. Molecular Psychiatry, 24(10), 1549-1564.

Ursini, G., Punzi, G., Chen, Q., Marenco, S., Robinson, J. F., Porcelli, A., Hamilton, E. G., Mitjans, M., Maddalena, G., Begemann, M., Seidel, J., Yanamori, H., Jaffe, A. E., Berman, K. F., Egan, M. F., Straub, R. E., Colantuoni, C., Blasi, G., Hashimoto, R., Rujescu, D., Ehrenreich, H., Bertolino, A., Weinberger, D. R. (2018). Convergence of placenta biology and genetic risk for schizophrenia. Nature Medicine 24, 792-801.

Pan, H. ${ }^{*}$, Oliveira, B. ${ }^{*}$ Saher, G. ${ }^{*}$ Dere, E., Tapken, D., Mitjans, M., Seidel, J., Wesolowski, J., Wakhloo, D., Klein-Schmidt, C., Ronnenberg, A., Schwabe, K., Trippe, R., Mätz-Rensing, K., Berghoff, S., Al-Krinawe, Y., Martens, H., Begemann, M., Stöcker, W., Kaup, F. J., Mischke, R., Boretius, S., Nave, K. A., Krauss, J. K., Hollmann, M., Lühder, F., Ehrenreich, H. (2019). Uncoupling the widespread occurrence of anti-NMDAR1 autoantibodies from neuropsychiatric disease in a novel autoimmune model. Molecular Psychiatry, 24, 14891501.

${ }^{*}$ Equally contributing authors 